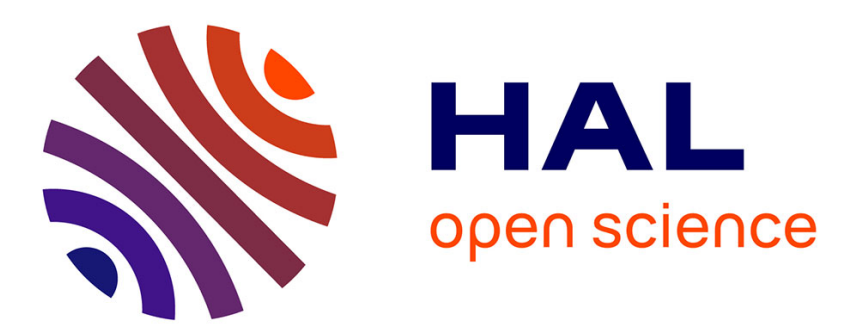

\title{
Tactical production planning for physical and financial flows for supply chain in a multi-site context \\ Yuan Bian
}

\section{To cite this version:}

Yuan Bian. Tactical production planning for physical and financial flows for supply chain in a multisite context. Operations Research [cs.RO]. Ecole nationale supérieure Mines-Télécom Atlantique, 2017. English. NNT : 2017IMTA0064 . tel-01719523

\section{HAL Id: tel-01719523 \\ https://theses.hal.science/tel-01719523}

Submitted on 28 Feb 2018

HAL is a multi-disciplinary open access archive for the deposit and dissemination of scientific research documents, whether they are published or not. The documents may come from teaching and research institutions in France or abroad, or from public or private research centers.
L'archive ouverte pluridisciplinaire HAL, est destinée au dépôt et à la diffusion de documents scientifiques de niveau recherche, publiés ou non, émanant des établissements d'enseignement et de recherche français ou étrangers, des laboratoires publics ou privés. 


\section{UNIVERSITE}

\section{BRETAGNE}

LOIRE

\section{Thèse de Doctorat}

\section{Yuan BIAN}

Mémoire présenté en vue de l'obtention du

grade de Docteur de l'École nationale supérieure Mines-Télécom Atlantique Bretagne Pays de la

$$
\text { Loire }
$$

sous le sceau de l'Université Bretagne Loire

École doctorale : MathSTIC

Spécialité : Informatique

Unité de recherche : Laboratoire des Sciences de Numérique de Nantes (LS2N)

Soutenue le 19 décembre 2017

Thèse $n^{\circ}:$ 2017IMTA0064

\section{Tactical production planning for physical and financial flows for supply chain in a multi-site context}

\footnotetext{
JURY

Rapporteurs : $\quad$ M $^{\text {me }}$ Safia KEDAD-Sidhoum, Maître de conférences HDR, Université Pierre et Marie Curie M. Pierre FÉNIES, Professeur des universités, Université Paris Nanterre M. Stefan Helber, Professeur des universités, Leibniz Universität Hannover, Allemagne

Examinateurs : $\quad$ M. Alexandre Dolgu, Professeur, IMT Atlantique campus de Nantes M. Jean-Laurent VivianI, Professeur des universités, Université de Rennes 1 Invités:

M. David LemoIne, Maître assistant, IMT Atlantique campus de Nantes M. Thomas G. Yeung, Maître assistant, IMT Atlantique campus de Nantes

Directrice de thèse : $\quad \mathbf{M}^{\mathrm{me}}$ Nathalie BostEL, Professeur des Universités, Université de Nantes
} 



\section{Acknowledgement}

First and foremost, I would like to express my sincere gratitude to my supervisors, Prof. Nathalie BOSTEL, David LEMOINE and Thomas YEUNG for all their contributions of time, ideas, and funding to make my Ph.D. experience productive and stimulating. Their continuous support, advice, caring, and trust throughout the past three and a half years was contagious and motivational for me, even during tough times in the Ph.D. pursuit. During my master internship and my Ph.D. program, I have benefited enormously from their valuable comments, their innovative approaches and serious attitude regarding research. I am also thankful for the excellent example they have provided as a researcher in Operations recherches that will guide and encourage me for my future career.

Special thanks goes to Prof. Safia KEDAD-SIDHOUM (Université Pierre Marie CURIE (Paris 6)), who teached me during my master program, supervised my master internship and whom became one of my thesis reviewers. Also a thank-you to Prof. Stefan HELBER (Leibniz Universität Hannover) for being part of my Generals committee, a thesis reviewer, and who provides many profound advises during an international workshop. And thank you to Prof. Pierre FENIES (Université Paris Ouest Nanterre La Défense) for his insightful comments from a financial point of view after reviewing my thesis. I am also grateful to other members of the defense committee, Prof. Alexandre DOLGUI (IMT Atlantique) and Prof. Jean-Laurent VIVIANI (Université de Rennes 1) for letting my defense be an enjoyable moment, and for your brilliant comments and suggestions that inspired me in many ways.

Our research works took part of the project RCSM which is financed by the French Competitiveness Cluster. In the context of this project, the collaboration with Prof. Jean-Laurent VIVIANI and Prof. Vincent HOVELAQUE (IGR-IAE of Rennes I) made our works possible and ensured its financial coherence with their knowledge and expertise in finance. I am grateful for all comments, advises and suggestions that I received for this thesis and I hope many success for the following project on this topic.

During the stay at IMT Altantique, the members of the team SLP of LS2N have contributed immensely to my personal and professional time. The team has been a source of friendships as well as good advice and collaboration. I would like to thank Fabien LEHUEDE, Prof. Olivier PETON, Odile MORINEAU, Naly RAKOTO, Guillaume MASSONNET, Laurent TRUFFET, Chams LAHLOU, Nadjib BRAHIMI for their helps, constructive discussions and considerations for the conviviality in the laboratory and to improve my research works. I am especially grateful to Axel GRIMAULT and Fabrice GAYRAUD who were always willing to provide their kind helps with my research and with my daily life. My gratitude also goes to Juliette MEDINA, Alex KOSGODA, Quentin TONNEAU, Clément FAUVEL, Thomas VINCENT, Tanguy LAPEGUE, Agnès LE ROUX, JeanGuillaume FAGES, Aleksandr PIROGOV, Oussama BEN-AMMAR, Olivier BACHOLLET, Gilles SIMONIN, Hélène COULLON for their kindness and sharing great time at IMTA and in Nantes. In addition, my dear Chinese friends, Mi ZHANG, Yier WU, Yulong ZHAO, Ning GUO, Jiuchun GAO, Xiao YANG, Junle WANG, Jing Li, Xing YAJING, Yuwei ZHU, Ka Yu LEE, Tianyu WANG, Zheng CHENG, Zhewei YU, Letian CHEN, He YUN who made the three years unforgettable. I am very much grateful for our friendships. I also recognize the support and assistance from Dominique 
MOREL, Isabelle LAINE and Anita NIEBROJ for the administrative procedures as well as from Delphine TURLIER of the Doctoral school of IMTA. For the non-scientific side of my thesis, I thank my teammates of basketball for the time playing together.

Last but not least, I owe my deepest gratitude to my dear family, especially, my grand parents, my mother and father, for their continuous encouragement, unconditional love and support as always in my life. They raised me with a love of science and supported me in all my pursuits. During my ten-year study in France, they gave up many things for me, cherished with me every great moment and supported me whenever I needed it. Also for my loving, supportive, encouraging, and patient beloved Ye whose faithful support during the final stages of this Ph.D. is so appreciated. 
Tian dao chou qin

- BIAN Jiawen 



\section{Contents}

\begin{tabular}{lll}
\hline 1 & General introduction & 11
\end{tabular}

$\begin{array}{lr}\text { General Introduction } & 11\end{array}$

\begin{tabular}{|lll}
2 & Research background, context and problem statement & 15
\end{tabular}

2.1 Notion of supply chain and supply chain management . . . . . . . . . . . . . . . . 15

2.2 Supply chain described by flows $\ldots \ldots \ldots \ldots \ldots \ldots$. . . . . . . . . . . . . 17

2.2 .1 Physical flow . . . . . . . . . . . . . . . . . . . . . 17

2.2 .2 Financial flow $\ldots \ldots \ldots \ldots \ldots \ldots$

2.2 .3 Information flow $\ldots \ldots \ldots \ldots \ldots \ldots$

2.3 Supply chain management and tactical planning . . . . . . . . . . . . . . . . . . . . 19

$2.3 .1 \quad$ Planning decisions in supply chain management . . . . . . . . . . . . . . . . 20

2.3 .2 Tactical planning, multi-site context and coordinations . . . . . . . . . . . . . 21

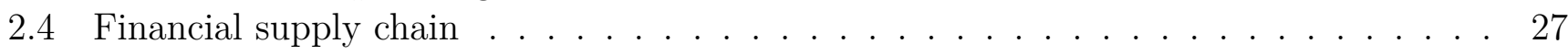

$2.4 .1 \quad$ Introduction to financial supply chain . . . . . . . . . . . . . . . . 27

$2.4 .2 \quad$ WCR: link between physical and financial flows . . . . . . . . . . . . . . . . . 29

2.5 Project "Risk, Credit and Supply Chain Management" . . . . . . . . . . . . . . . . . . 33

2.6 Problematic and contribution $\ldots \ldots \ldots \ldots \ldots \ldots$

\begin{tabular}{|lll}
\hline & Integration of working capital requirement financing cost in EOQ model & 37
\end{tabular}

3.1 Introduction $\ldots \ldots \ldots \ldots \ldots \ldots \ldots \ldots$

3.2 The Economic Order Quantity model . . . . . . . . . . . . . . . . . . . 37

3.2 .1 The EOQ model . . . . . . . . . . . . . . . . . . . . . . 37

3.2 .2 Financial aspects considered in the literature . . . . . . . . . . . . . . . . . . . 39

3.2 .3 Positioning the work of this chapter . . . . . . . . . . . . . . . . . . . . . 43

$3.3 \quad$ EOQ based profit maximization model, $E O Q_{P(W C R)}$. . . . . . . . . . . . . . . . . . 43

3.3 .1 OWCR modeling $\ldots \ldots \ldots \ldots \ldots \ldots$

3.3 .2 Assumptions . . . . . . . . . . . . . . . . . . . . . . . . . . . . . . . . . 44

3.3 .3 Parameters et decision variables . . . . . . . . . . . . . . . . . . . . . . . . . 44

3.3 .4 OWCR formulation $\ldots \ldots \ldots \ldots \ldots$. . . . . . . . . . . . . . . . . . . . . . 44

3.3 .5 Objective function $\ldots \ldots \ldots \ldots \ldots \ldots$

3.4 Optimal solution and structural properties $\ldots \ldots \ldots$. . . . . . . . . . . . . 47

3.4 .1 Optimal solution $\ldots \ldots \ldots \ldots \ldots \ldots \ldots$

3.4 .2 Comparison with classic EOQ based-formula with the cost of capital . . . . . 53

3.5 Numerical analysis $\ldots \ldots \ldots \ldots \ldots \ldots \ldots \ldots$

3.5.1 Comparison betweens results obtained by the proposed model and the classic

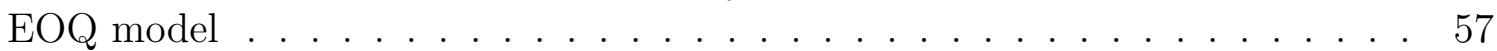

3.5 .2 Illustration of the trade-off between costs $\ldots \ldots \ldots \ldots$. . . . . . . 57

3.5.3 Sensitivity of $Q^{*}$ to varying parameters in the classic and proposed $E O Q$ models 58




4 Dynamic lot-sizing based discounted model considering the financing cost of work$\begin{array}{lc}\text { ing capital requirement } & 61\end{array}$

4.1 Introduction . . . . . . . . . . . . . . . . . . . . . . . . . . 61

4.2 Dynamic lot sizing models . . . . . . . . . . . . . . . . . . . . . . 61

4.2.1 The uncapacitated Lot-Sizing (ULS) model and mathematical formulations . . 62

4.3 The discounted cash flow model of the DLS problem . . . . . . . . . . . . . . 65

4.3.1 Mathematical Formulation of the discounted cash flow model for DSL problems 66

$4.3 .2 \quad$ Literature review of discounted cash flow model for the DLS problem . . . . . 67

4.4 ULS-based profit maximization DCF model . . . . . . . . . . . . . . . . . 68

4.4 .1 OWCR modeling . . . . . . . . . . . . . . . . . . . . . 68

4.4 .2 Assumptions . . . . . . . . . . . . . . . . . . . . . . . . . . . . . . 69

$4.4 .3 \quad$ Physical and financial flows illustration . . . . . . . . . . . . . . . . . . . 69

4.4 .4 Parameters et decision variables . . . . . . . . . . . . . . . . . . . . . . . . . . . . . . . . . . . . . . .

4.4 .5 Mathematical formulation . . . . . . . . . . . . . . . . . . 71

4.4 .6 Objective function $\ldots \ldots \ldots \ldots \ldots \ldots$

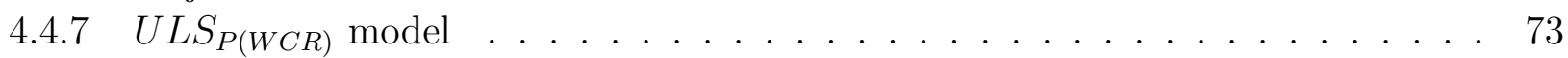

4.5 Solution method $\ldots \ldots \ldots \ldots \ldots \ldots \ldots$

4.5 .1 Zero-Inventory-Ordering property . . . . . . . . . . . . . . . . . . . . . . . . . . . . . . . . .

4.5 .2 Proof of Theorem . . . . . . . . . . . . . . . . . . . . . . . . . . . . . . . . . . . . . . . . . . . .

4.5 .3 Algorithm description . . . . . . . . . . . . . . . . . . . . 80



$4.6 .1 \quad$ Production program comparisons . . . . . . . . . . . . . . . . . . 82

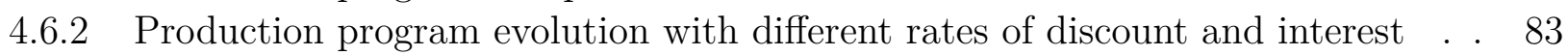

$4.6 .3 \quad$ Production and financial cost comparisons . . . . . . . . . . . . . . . 84

$4.6 .4 \quad$ Program evaluation with different purchasing costs and delays in payment to

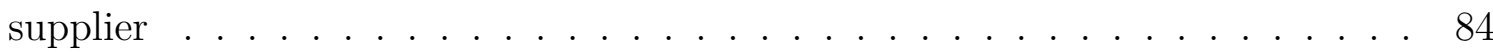

$4.6 .5 \quad$ Program evaluation with different production unit costs . . . . . . . . . . . . . 85

$4.6 .6 \quad$ Program evaluation with different delays in payment from client . . . . . . . . 86



5 Multi-level uncapacitated lot sizing based discounted model considering the fi$\begin{array}{ll}\text { nancing cost of working capital requirement } & 87\end{array}$

5.1 Introduction $\ldots \ldots \ldots \ldots \ldots \ldots \ldots$. . . . . . . . . . . . . . . . . . . 87

5.2 The multi-level uncapacitated lot sizing-based models . . . . . . . . . . . . . . . . . 87

$5.2 .1 \quad$ MRP and the multi-level lot-sizing problem . . . . . . . . . . . . . . . . 87

5.2 .2 The description of the MLLP problem and associated mathematical formulation 88

5.2 .3 Review of the multi-level problem . . . . . . . . . . . . . . . . . . . . . . . . . . . 90

5.3 Two-level discounted models with WCR costs . . . . . . . . . . . . . . . . . . . . . 92

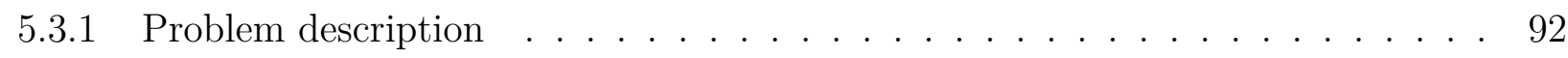

5.3 .2 OWCR model in two-level case . . . . . . . . . . . . . . . . . . . . . . . . . . . . . . . . . . . . . . . . . .

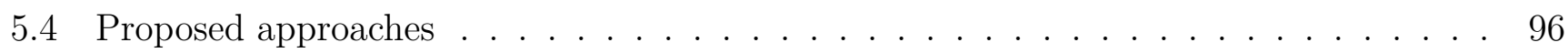

5.4 .1 Mathematical formulation . . . . . . . . . . . . . . . . . . 96

5.4 .2 Sequential approach . . . . . . . . . . . . . . . . . . . . . . . . . . . . . . . . . . 97

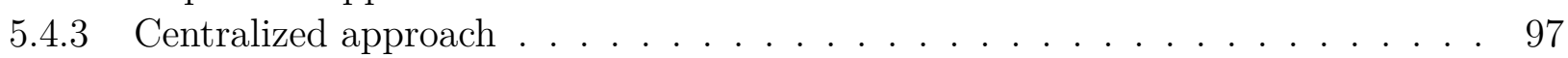

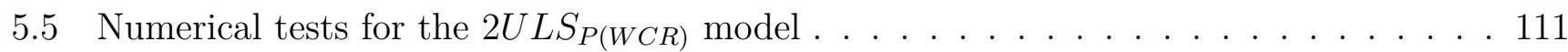

5.5 .1 Test 1: production program comparisons . . . . . . . . . . . . . . . 112

5.5 .2 Test2: production program evolution with different ratios between two-level discount rates . . . . . . . . . . . . . . . . . 114

5.5.3 Test3: program comparisons with different payment delays . . . . . . . . . . 115

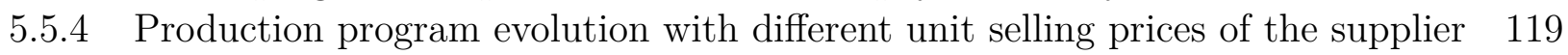


5.6 Generalization to multi-level problem . . . . . . . . . . . . . . . . . . . . . 120 $5.6 .1 \quad M L L P_{P W C R}$ model and centralized approach . . . . . . . . . . . . . . . . . . 121 5.6 .2 WCR model in multi-level . . . . . . . . . . . . . . . . . . . . . . . . . . . . . 121

5.7 Conclusions . . . . . . . . . . . . . . . . . . . . . . . . . . . . . 124

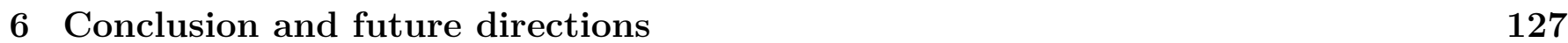

\begin{tabular}{|r|r}
\hline Conclusion and future directions & 127
\end{tabular}

\begin{tabular}{lll}
\hline 7 & Résumé en français & 131
\end{tabular}

\begin{tabular}{ll}
\hline Résumé en français & 131
\end{tabular}

\begin{tabular}{lr}
\hline List of tables & 137
\end{tabular}

\begin{tabular}{lr}
\hline List of figures & 140
\end{tabular}

\begin{tabular}{lr}
\hline Bibliography & 150
\end{tabular} 



\section{General introduction}

Since the 1980s, supply chain management (SCM) has supplanted the traditional production and logistics management problems which are based on a single entity. Subcontracting and outsourcing, mergers, acquisitions and alliances among companies entail a considerable complication at both intraand inter-organizational levels for all companies. Understanding the optimal mechanism within the entities involved in the chain and ensuring an overall performance are recognized as the ultimate and ideal goal that is extremely difficult to reach, but still be pursued by the academics and practitioners over decades.

There are various study fields of the SCM, such as the fields of classic logistics, operational research, management control, information technology, or even law. The SCM issues always fall within a global hierarchical problems. Indeed, in the literature, the SCM is described by three levels: strategic, tactical and operational levels. Our problem focuses on the study of tactical production planning problems in the SCM.

Traditionally, tactical production planning aims at determining the production quantity over the planning horizon during each period. It is often known for its significant influence on the customer service quality and production-related operation costs. Two main classes of the model distinguish the problem based on continuous or discrete time, for instance, Economic Order Quantity (EOQ) model of Harris (1913) for constant demand over continuous time and Uncapacitated Lot-Sizing model (ULS) of Wagner \& Whitin (1958) for time-varying demand over discrete time. However, the traditional production planning is determined following the well-known Manufacturing Resource Planning (MRP II) logic for medium-term objectives that only consider the physical flow of goods. This decision fails to reflect the Net Present Value (NPV) of cash flow due to planned operations in the real world market system as indicated by Helber (1998). Therefore, financial aspects should be integrated in the traditional production planning model.

This thesis mainly focuses on the integration of the Working Capital Requirement (WCR) aspect in the production planning process. Recently, the WCR management draws an increasing attention in the finance department of companies, especially since the financial crisis. In the context of the financial crisis, companies need more free cash flow to efficiently react against all uncertainties to ensure solvency. According to the Ernst \& Young annual Working Capital Management (WCM) report of 2012 devoted to the leading 1000 US companies in 2011, on average, Net Working Capital (NWC) of $\$ 330$ billion dollars is unnecessarily immobilized (see in Ernst (2012)). This range of cash opportunities corresponds to their aggregate sales of $3 \%$ and $6 \%$ respectively. Buchmann et al. (2008) also stress that savings on the WCR as a potential source of cash to fund company's growth is often neglected in practice. Suboptimal WCM not only reduces potential gains, but also raises company's 
risk. A company should carefully manage its WCR in order to ensure financial liquidity or reduce the insolvency risk. Finally, optimal WCM can unlock internal capital and provide financial resources for financially-constrained firms. Especially during the last financial crisis period, bank loans were extremely difficult to be obtained by companies especially those in the development phase (see in Wu et al. (2016)). Many companies suffer from lack of credit and insufficient working capital. Small suppliers have to accept unfavorable payment terms from their customers, which exacerbates their financial situation. Moreover, tight or unavailable bank credit reduces the working capital level of these companies. As a result, some companies have to suspend their operations which can disrupt the whole supply chain as reported in Benito et al. (2010) and Ernst (2010).

In spite of its importance for the firm (and more generally the supply chain) performance and survival, the WCM has been neglected by the literature on production planning. More generally, as stated by Birge (2014), "operations management models typically only consider the level and organization of a firm's transformation activities without considering the financial implications of those activities". The separation of operational decisions (minimizing operational costs or maximizing operational profits) and financial decisions (minimizing financial costs or maximizing financial profit) was theoretically grounded on the famous Modigliani \& Miller (1958) theorem and practically by the functional organization of companies. However, in recent years, the literature on operation and supply chain management, following the original work of Babich \& Sobel (2004), became aware of the fact that financing and operational problems are imbricated and that optimizing the two dimensions globally can improve the global performance of the company as shown in Chen et al. (2014). A recent review of the literature based on a risk management framework has been proposed by Zhao \& Huchzermeier (2015). Nevertheless, in the production planning field majority of models typically ignore the financial consequences of production planning decisions. In an attempt to fill this gap, the aim of the paper is to explicitly introduce the WCR dimension in a tactical production planning model.

The general structure of the manuscript is presented as follows:

The first chapter gives a detailed analysis of concepts on the supply chain in the literature, classifications and challenges. It allows us to understand the complexity of the supply chain. Then, financial supply chain management and its main approaches are presented. Furthermore, the third part will focus on the working capital and working capital requirement management, while the fourth part will describe our problematic within the context of the RCSM project (Risk, Credit and Supply chain Management) and the main contributions of this thesis.

In the second chapter, we tackle the single-level problem considering the financial cost of the WCR in the EOQ context. A state of the art of financial aspects which have been integrated into the EOQ model in the literature is first provided. This review shows that only few works have considered the working capital requirement in the past and absence of consideration of working capital requirement in the tactical production planning context. Secondly, we focus on establishing a suitable model of the WCR in single-level, single-product and for constant demand over continuous-time planning horizon case. We further integrate it into the classic EOQ model. Analytical analysis are executed at the end of the chapter.

In the third chapter, we consider the financing cost of the WCR based on the dynamic lotsizing (DLS) model for time-varying demand. Firstly, a review of the DLS models in the production planning literature is given. Then, a generic modeling of WCR is established in the ULS context which allows us to follow the evolution of the WCR during the planning horizon. Second, a ULS-based discounted cash flow model is built considering the financing cost of the WCR in single level, single product, with infinite production capacity and constant parameter values. Inspired by the classic ULS, the Zero-Inventory-Ordering (ZIO) property is proven to be valid in this problem. With this property, a dynamic programming-based algorithm is constructed. A numerical analysis is preformed and shows the difference between the classic ULS model and the proposed model in terms of the optimal production program. Moreover, the sensitivity of the proposed model to the variation of 
financial parameters is also investigated.

In the fourth chapter, we first extend the previous single-level model to a two-level (suppliercustomer) model and further generalize it to a multi-level case based on the Multi-level Lot-sizing model (MLLP). The WCR model is modified to adapt this multi-level scenario. Considering the OWCR cost in the multi-level case, sequential and centralized approaches are proposed to solve both the two-level and the multi-level problems with a serial chain structure. The ZIO property still naturally stands in the sequential approach. It is further proven to be valid in centralized approach. The property equally allows us to establish a dynamic programming-based algorithm. Finally, the differences in optimal solutions obtained by the sequential and the centralized approaches are analyzed. 



\section{Research background, context and problem statement}

Since the 1990s, supply chain management has been largely addressed by scholars and practitioners. Related literature is significantly rich and diversified on its conception, optimization and application. This chapter is dedicated to positioning our tactical planning problem within the recent trends of supply chain management.

This chapter is divided into four parts: the first part is devoted to the analysis of concepts of the supply chain in the literature, classifications and challenges. It allows us to understand the complexity of the supply chain. The second part will then present financial supply chain management and its main approaches. The third part will focus on working capital and working capital requirement management, while the fourth part will describe our problem within the context of the RCSM project (Risk, Credit and Supply chain Management) and the main contributions of this thesis.

\subsection{Notion of supply chain and supply chain management}

Development of industry, growth of product complexity and the globalization have led to the supply chain being more sophisticated to manage which requires more investment and special expertise in supply chain management (SCM). It involves the trend of outsourcing for logistics services, which were initially carry on by the company itself, to external service providers since 1980s. Furthermore, the lean management approach, imported from the USA and Asia to Europe, encouraged companies to focus on their core competencies and to seek service providers for downstream processes, including logistic activities. Therefore, the organizational structure for the logistic activities in the 1990s tended to an external integration scheme. However, this evolution in logistics brought a reflection facing a more complex chain to manage with an inter-organizational consideration.

In literature, many definitions of supply chain management are proposed (Christopher (1992); Lee \& Billington (1993); Ganeshan \& Harrison (1995); Genin (2003)). For example, Cooper et al. (1997) defined it as "a key management, integrated business processes from original suppliers to ultimate user, that provides products, associates services, and information that creates additional value for customers and other stakeholders." More recently, the Council of Supply Chain Management Professionals provided the official definition of SCM:" Supply chain management encompasses the planning and management of all activities involved in sourcing and procurement, conversion, and all logistics management activities. Importantly, it also includes coordination and collaboration with 
channel partners, which can be suppliers, intermediaries, third party service providers, and customers. In essence, supply chain management integrates supply and demand management within and across companies."

Moreover, Féniès (2006) defined it as "an open set crossed by flows, composed of entities and various autonomous actors who use limited resources (capital, time, equipment, raw materials ...) and who coordinate their actions through an integrated logistics process in order to improve their collective performance (satisfaction of the final customer, global optimization of the supply chain functioning) as well as their individual performance (profit maximization of an entity). This definition shows that a supply chain can be described with the three different sets:

- a network composed of physical entities (factories, workshops, warehouses, distributors, wholesalers, retailers, etc.) and autonomous organizations (firms, subsidiaries , ...);

- an open set crossed by flows (financial, material, information, ...);

- a set of activities regrouped in an integrated logistics process, the layout of which constitutes an intra and inter-organizational value chain.

Following this definition, the logistics chain can first be described as a network of physical entities and autonomous organizations. Thus, Lee \& Billington (1993) define the supply chain as "a network composed by production sites and distribution sites that procure raw materials, process and distribute them to the consumer". Moreover, both service and production entities exist in the supply chain. Genin (2003) thus proposes that: "A supply chain is a network of geographically dispersed organizations or functions on several cooperating sites to reduce costs and increase the speed of processes and activities between suppliers and customers." There are two different criteria that differentiate the way of describing the supply chain with entities. First, some consider each product as a chain and associated infrastructures (see in Pimor (2001)). Thus, Rota et al. (2001) define the supply chain of a product as the set of all entities from the first supplier to the ultimate customer of that product. The second distinguishes logistic chains according to the legal aspects related to their entities. Consequently, Colin (2004) proposes that the supply chain can be separated into an internal supply chain and an external supply chain. In the same way, Genin (2003) indicates both the inter and intra-organizational supply chain. In the case of legally linked entities, they form an "intra-organizational" supply chain that involves a single legal entity, mono or multi-site. Otherwise, the supply chain is considered as "inter-organizational". The difference between these types of supply chains essentially lies in the decision-making process. Thus, the management of "inter-organizational" supply chains cannot ignore the complexity associated with the need for collaboration between different actors, contrary to an intra-organizational supply chain where it is easy to assume a centralized decision-making mode.

The logistics chain can also be defined by the activities and processes that it generates and which it supports. The definition of Fenies et al. (2004) describes the logistics chain from a set of activities grouped together in an integrated logistics process, the arrangement of which constitutes an intra and inter-organizational value chain. The definition of logistics chains by the process approach consists in describing the activities between firms and in the company that satisfy the end customer (Lee \& Billington (1993), Beamon (1998) and New \& Payne (1995)). Tchernev (1997) thus presents an extension of the logistics process concept for any logistics system and defines the logistics process as a set of ordered activities with the objective of controlling and managing material flows through the logistic system. Then, it allocates resources in the logistics system in order to ensure best level of services at the lowest cost. As given in Vowles (1995), the activities in the logistic process includes:

- Ordering

- Purchasing

- Production

- Control

- Conditioning

- Inventory holding 


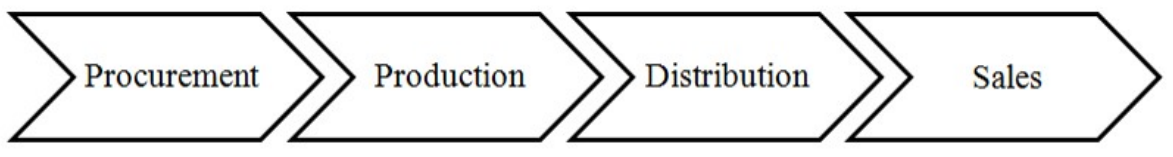

Figure 2.1 - Activities in the logistic process in the supply chain

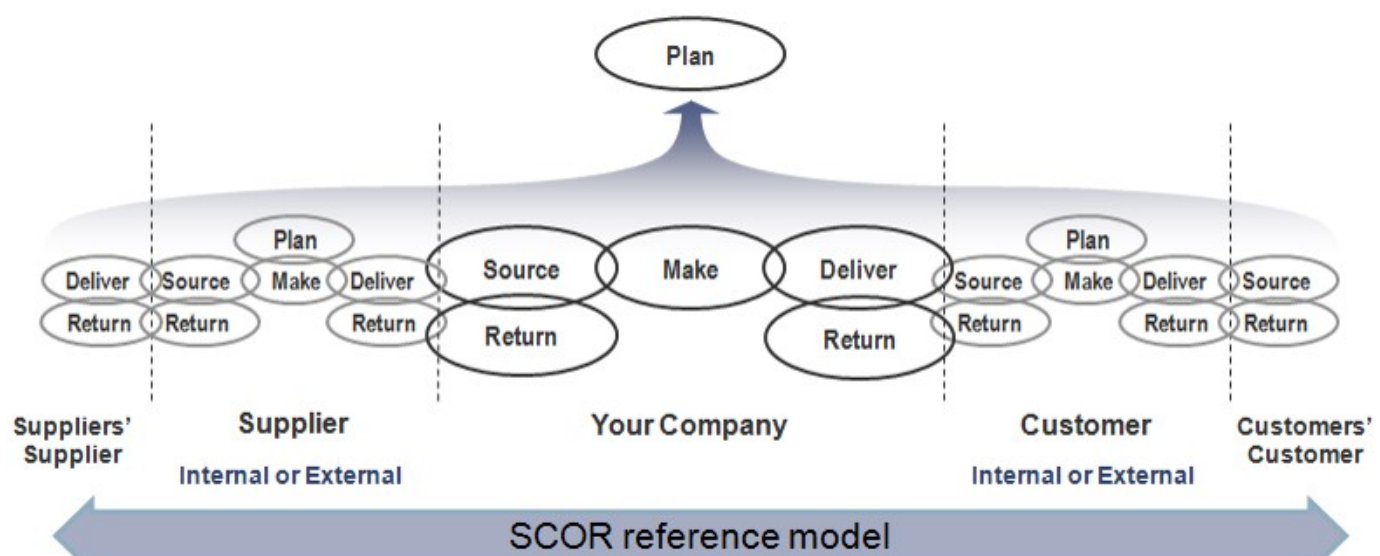

Figure 2.2 - Inter-organizational process in the supply chain

- Distribution

- Transport

Other classification of activities can be found, for example, in (Stadtler (2005), see Figure 2.1) and in Supply chain council proposition (see Figure 2.2).

\subsection{Supply chain described by flows}

The supply chain can also be described with the three flows that co-exist and pass through it. The term "flow" has been largely used since Forrester (1997) published his first version of work which deals with industry dynamic problems. In this work, the company is modeled using flows. It allows us to understand the connections between different entities. The author considers the interactions which are described in six flows: (1) material flow, (2) money flow, (3) order flow, (4) information flow, (5) human resource flow and (6) financial capital flow. However, the flows used to characterize a company are various in the literature. Cooper et al. (1997) include flows of materials, goods and information in one direction, and flows via financial and information flows in the opposite direction. Pfohl \& Gomm (2009) define four different types of flows: information, financial resources, goods and rights. Croom et al. (2000) distinguish the flows in goods, financial resources, human resources, information, knowledge and technology. Dherment-Férère (2007) points out that "there are two kinds of flows within an enterprise: logistic flows and financial flows". These definitions diverge on the definition of flows, on the number of considered flows and on its propagation direction. In the following chapters of this thesis, we consider the flows in three aforementioned categories: the physical, financial and information flows.

\subsubsection{Physical flow}

The first flow discussed here is physical which includes activities of raw material purchasing, product transformation from the raw material and delivery of the products. Traditionally, the objective of managing this flow is to satisfy client's demand at the lowest cost. In the literature, several 
definition are proposed in different points of view.

— "A physical flow in industrial production is a movement, in time and space, of material elements, from the reception of the raw materials or semi-finished products, until the delivery to the customer for satisfying its demand" Biteau \& Biteau (2003);

- "The physical flow is tangible and material contrasting with the flow of services that is intangible and immaterial" (Dherment-Férère (2007));

- "The physical flow can be described as a set of units circulating in space, on a surface, on a plane, on a curve, or on a straight line according to a precise law. The processes duration for processing these flows allows to plan the productivity of a system, while the connection between quantity and time describes the productivity of the system related to this physical flow" (Tchernev (1997)).

\subsubsection{Financial flow}

The financial flow, consisting of the cash flows of the supply chain, aims to satisfy the actors who contribute to the functioning of the supply chain (legal entities, human resources, shareholders, banks, etc.). It is the monetary counterpart of physical flow. This flow is also known as "cash flow". It is always generated by the same activities that generate physical flows, such as production, transport, storage or recycling. Financial flows are thus not independent. It is often correlated with physical flows because it is the reception of the goods that triggers payment. However, it can also be not correlated to the physical flow, for example, with the payment of a deposit. With regard to the supply chain, the financial flow is mainly unidirectional, i.e. from the final customer to the supplier of the highest rank. Nevertheless, at company level, the flow is bi-directional including incoming (e.g. revenue) and outgoing (e.g. cost) cash flow. Different cash flow optimization objectives are addressed in the literature. Cash flow optimization may allow, a priori, to reach shareholders' satisfaction for entities in the supply chain and to improve its overall functioning (Shapiro (2006)). Dematerialized payment impacts the cash flow for accomplishing long-term objectives of shareholders in entities of the supply chain, the medium-term customer credit policy and short term cash flow scheduling according to Lysons \& Farrington (2006).

Some limits of managing the financial flow are identified by Hohmann (2004) because it does not reflect the problematic in a coherent way. Féniès (2006) indicates that, even if using monetary units, analytic accounting models produce information on the levels of profits and costs generated by the activity of the physical flow, but do not allow to measure the associated cash flow level. It is because they do not take into account the specification of the calculated charges and products nor the payment deadlines. A cash balance or a financing plan is more suitable for financial flow management. Thus, financial flows have a dynamic aspect, which can not be captured by the traditional accounting approaches, which certainly allow to have a precise vision of the economic reality of a structure but at a given and fixed moment. In contrast, the cash flow principle is to ensure the availability of all payments (see in Cooper et al. (1997)). Also, when we use the term of financial flows, we mainly refer the flows related to the exploitation cycle. Non-operating financial flows have a lesser occurrence in firms, following strategic level decisions, with relatively high predictability. Physical and financial flows are jointly linked thanks to an information flow whose quality guarantees their translation. Thus, financial flows are only the tangible representation and final result of the intellectual and physical action of the employees.

\subsubsection{Information flow}

The information flow represents all transfers or exchanges of data between the various actors in the supply chain, so that they can respond to the needs expressed by the order of the final customer. The information flow is bidirectional and can possibly be linked to the physical and financial flow. 
Fawcett \& Magnan (2001) indicate that information flows "coordinate physical and financial flows between each node of the supply chain network and thus allow a global coordination. Kyriakopoulos \& De Ruyter (2004) propose the notion of internal and external information flow to characterize the origin of the flow. Moreover, the information flow can be horizontal, vertical, or a combination of both. "Vertical flows of information correspond to hierarchical planning. Horizontal information flows can exist between two entities who easily and quickly use local information, for example to control the effects of a machine failure" (Meyers \& Tucker (1989)).

Information flows must be reliable and traceable in order to ensure the intelligibility of their content and avoid any form of distortion. Reliability refers to the fact that information does not suffer from any failure: it should be accurate, correct, up-to-date and controllable. The problems of inherent quality of the information flow and its propagation within the supply chain have been largely investigated in the literature. Entitled "the Bullwhip effect", it refers to the phenomenon of information distortion which is propagated along the supply chain by expanding and resulting in instability in trade and economic losses according to Lee et al. (1997).

Regardless of the hierarchical level of the logistics system, the information flows contain necessary data for the planning and management of all the activities. The following information must be available:

- clear information on resources for executing this activity (the state of these resources, the necessary knowledge and data for associated operations, the local rules for their steering);

- information on the status of activities that depend on this activity;

- information on the status of this activity concerning all dependent activities;

- information on the status of this activity concerning the management system of the flexible production / storage unit or feedback;

- the global rules for the steering and management of all activities related to the considered activity.

\subsection{Supply chain management and tactical planning}

The works of Stadtler (2005) and Kilger et al. (2015) present a clear understanding and summarize the essential knowledge on SCM resulting from the very rich and wide-ranging operations research field. Stadtler (2005) defines SCM as "an approach that allows the integration of organizational units along a supply chain and the coordination of physical, financial and information flows in order to satisfy the final consumer and improve the competitiveness of supply chain". The author proposes to consolidate the necessary methods and tools for the success of an SCM approach within the Supply Chain Management house as presented in Figure 2.3.

- The roof of the SCM house is the objective of SCM approach, that includes both the final customer's satisfaction and the competitiveness improvement of the Supply Chain. These two objectives are held on two pillars: supply chain members integration and the processes coordination;

- The first pillar of supply chain members integration consists of three "blocks". They include the integration of partners selection, the supply chain network organization/inter-organizational collaboration and the leadership situation in the supply chain;

- The second pillar consists of the coordination between three approaches. The use of information/communication technology accelerates the exchange between partners and restrain the bull-whip effect. Process management tears down the barriers between business function and organizations. Lastly, advanced planning reinforces the strength of transactional enterprise resource planning (ERP) in planning area.

- These two pillars are based on conceptual and academic foundations from several fields (logistics, operations research, marketing, organizational theory, etc.). 




Figure 2.3 - House of supply chain management (Stadtler, 2005)

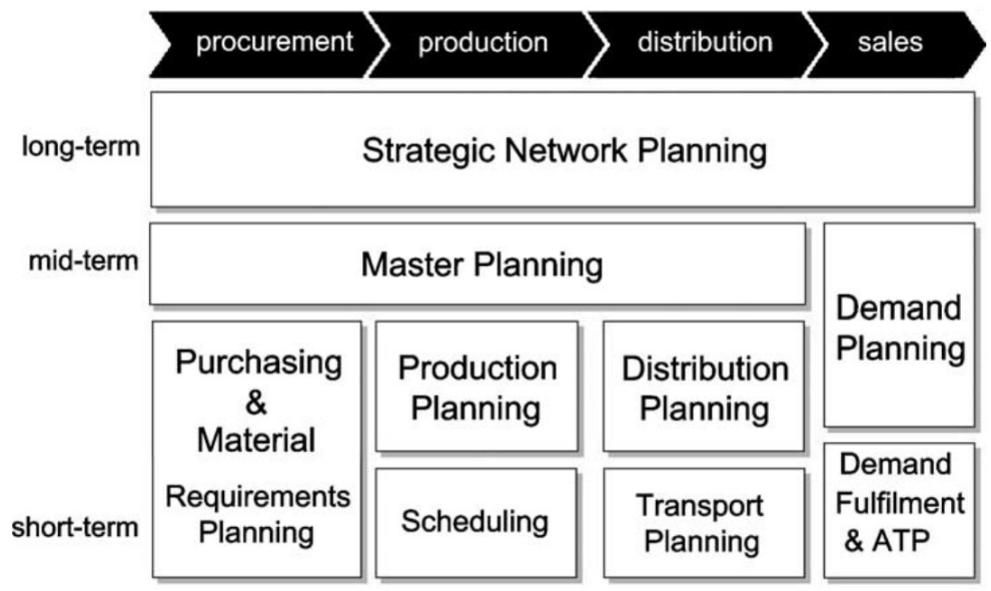

Figure 2.4 - Software modules covering the supply chain planning matrix (Meyr et al. (2008))

\subsubsection{Planning decisions in supply chain management}

Various decisions of activities planning in supply chain concern improving supply chain performance and efficiency. Proposed as a solid reinforcement of ERP system, advanced planning (or advanced planning and scheduling (APS)) system provides analysis and planning of logistics and manufacturing. Meyr et al. present a two-dimension classification of these plannings in the "supply chain matrix" (see Figure 2.4):

- the level of the managerial decision making involved and the time during which the decision will have an impact on the future development of the supply chain. Following the principles of hierarchical production planning, three planning levels are used: "long-term/strategic", "mid-term/tactical" and "short-term/operational",

- the supply chain process involved. Four different processes are identified: procurement, production, distribution and sales.

Similar classification of levels is also illustrated in Genin (2003) as three levels of planning. Industrial planning have been recognized as a major problem existing at all three levels. It aims at delivering ordered products to customers at the right time, at the lowest cost and to the right place. In other words, the challenge is to find a trade-off between customer satisfaction rate and different costs generated by production-related activities (production, storage, etc.). As it is integrated in production management, the industrial planning is thus divided into these three hierarchical levels. 
Subsequently, long-term and short-term level decisions and plannings will be briefly presented. Then, the problematic at the tactical level will be discussed in detail in the following sub-chapter.

\section{- Strategic level:}

Strategic decisions always concern the general company management over the long term horizon (conventionally, more than 18 months) in order to make decisions on the company's major policy. It thus implies a decision on its portfolio of activities which will involve necessary and stable resources to ensure the achievement of these decisions. The stable resources include machines as well as manpower. The decisions may concern their purchases or hiring, replacements, discard or layoff, transfers, locations, etc. It may also concern production information (formalized management procedures, technical databases, etc.). All these decisions will then be implemented at lower levels.

Strategic planning aims to satisfy the market as a whole within the framework of the general strategy of the company. More specifically, it seeks to determine its growth, development and profitability objectives. Its primary responsibility is to find a global strategy to achieve long-term objectives through substantial changes in production capacities (new production units, new locations, adoption of new technologies, etc.).

- Operational level:

Operational decisions should respect decisions at the tactical level and further ensure necessary daily flexibility to handle expected demand fluctuations and resource availability based on forecasting. Therefore, implementation at the operational level requires a complete disaggregation of the plan defined at a higher hierarchical level. Regarding production management, operational decisions include:

- Inventory management, which ensures the availability of raw materials and components;

- Scheduling, which consists of a detailed programming of the mobilized resources (operators, equipments and tools) for execution of necessary operations for basic production of goods or services over a horizon of no more than a few days, within a time framework of minutes.

The operational planning is always determined over a daily or weekly horizon. It closely monitors and controls physical flow in order to ensure the availability of the products at each line according to the conditions defined at the tactical level. This thus involves production scheduling on a day-to-week basis which means determining the starting time of tasks of different resources in order to guarantee punctuality of the production and the accurate quantity of products decided at the tactical level. For this reason, this approach is traditionally called "planning by timing". The associated problematics at the operational level are traditionally addressed in the scheduling area.

\subsubsection{Tactical planning, multi-site context and coordinations}

The tactical level connects the strategic and operational levels. The decisions at this level are made for a medium term horizon (6 to 18 months). As indicated in Tchernev (1997), three types of planning problematics exist at this level which are procurement planning in upstream, production planning in the production unit and transport planning in downstream. It is important to notice that all these decisions should fulfill the global framework defined by the strategic decisions.

The production planning aims at determining the quantities of products to be manufactured over a medium- or short-term horizon (priority planning) and to decide the amount of resources to be assigned (capacity planning) in order to meet the operational objectives in the best quality, volume, location, duration and cost. The capacity of facilities is limited and will not be significantly improved over the planning period. Therefore, the only available solution left to the manager is to optimize the production quantities using available or additional production resources (e.g. overtime, temporary hiring...), the variation of resource quantities, or searching external recourse. 


\section{Practical approaches}

In the literature, tactical planning is considered as hierarchical approach as proposed by Vollmann et al. (1997). The authors decompose tactical planning into three levels :

- Sales and Operations planning (S\&OP).

- Master production schedule (MPS)

- Material Requirement Planning (MRP)

First, the objective of S\&OP is to transform the business plan into a forecasting production volume for each major product family of the company. It is carried out not only based on sales forecasts, but also based on markets and economic situation. It will be updated at least every month by the various managers of the company (sales, production, supply chain, marketing, $R \& D$...). It integrates plans of all sectors of the company. Indeed, the S\&OP is always used to coordinate around a common axis the different strategies of company's services. Nevertheless, S\&OP reflects what the company wants to produce and not the full capacity of production.

Since the time required for the implementation of the S\&OP decisions is generally long, its elaboration is therefore based on sales forecasts and its horizon is 12 to 18 months. The traditional elaboration is based on graphic methods or linear programming models e.g., in Giard (2003), Herzer (1996), Lamouri \& Thomas (1999) and Baglin et al. (2005). The graphic methods are easy to understand and to use. They proceed by successive cost evaluation calculations and allow to identify different integrated plans that are valid but whose costs are not necessarily the lowest. However, its quality is often based on experience and common sense. On the contrary, the linear models optimally manage the strategies for a set of parameter inputs. Although, the user should be concise of the "forecasting" nature of these parameters because it may lead to a biased result that is far from the optimum and even influence the optimal strategy. Regarding the importance of S\&OP decision, its robustness should take priority over its optimization.

Therefore, the S\&OP provides a capacity and sales planning which leads to the next level of tactical planning to define a master production schedule (MPS). Generally, MPS is established every month over a period of time from three to six months for the key product, commonly the finished product. It determines the quantities to manufacture for each period of the horizon (day) by each plant with precise planning of required resources available in the plant. Compared with planning obtained by S\&OP, planning of MPS is established with a smaller time unit, e.g. day. Furthermore, the MPS planning focus on allocation of critical resources in the system for a local objective.

The primary objective of the MPS is therefore to ensure the on-time delivery of customer orders. The second is the optimal resource allocation. The MPS evaluates the product availability for client's demands. It thus plays a major role in the functioning of an integrated planning and control system for production and inventories, since it establishes at each period the balance between the company's resources and the demands to meet. Thus, Tchernev (2003) considers that "the MPS is at the center of tensions generated by disruptions in the company, such as production delays or in its external environment, such as demand changes".

Different from the MPS that considers only one level planning, the MRP allows a multi-level manufacturing planning of finished products and its components over the same planning horizon. The bill of material(BOM) represents the product structure and is introduced in the MRP method. According to the BOM, The resource required of each component are calculated from the bottom level of the BOM to the level of the component considering the associated coefficients. In addition to planning, the MRP is also used for system control to manage manufacturing processes and indicate "directions" for resource allocations. Ultimately, the MRP determines necessary components or raw materials to prepare in order to accomplish the MPS, item quantities to purchase and manufacture regarding the inventory level and the cycle and quantities of orders as well as the staring time of production.

During the planning elaboration with MRP, the delivery delay, production lead time and assembly duration are taken into account. The quantities determined by the MRP are strongly correlated 
with the MPS decisions. Even small inaccuracies in MPS planning may be significantly amplified through the MRP system. Even though MRP principle is easy to understand, the difficulties always appear during its implementation in an industrial environment. The reason is that, in practice, a considerable number of calculations based on a complex product BOM will be generated. It thus generates thousands of manufacturing and purchasing orders that must be precisely controlled and pursued. In fact, when quantities and timings of production and control activities are fixed, it is then possible to schedule the activities. Thus, the MRP is considered as the interface of detailed operation planning and scheduling.

Initially, the MRP did not take account the external constraints and planned the needs only on the basis of demand or demand forecast considering:

- an unlimited production capacity always capable of supplying,

- no uncertainties (breakdowns, delays of delivery ...)

In 1971, Orlicky defined the MRP I method, which takes into account finite capacities. Then, in 1984, Wight launched the foundation of the MRP II (Manufacturing Resource Planning) method by extending the concept to management resources planning and the integration of financial and logistical data (Wight (1995)). In MRP II framework, the capacities of production, supply, subcontracting, storage, distribution and also financial resources are taken into account Genin (2003).

\section{Multi-site tactical planning}

The previous hierarchical structure is limited to a single-site description of tactical planning. In supply chain management, tactical planning attempts to further ensure a "horizontal synchronization" of the chain. At the MPS level, the synchronization is achieved by a process called Distribution Requirement Planning (DRP). This method is used in business administration for planning orders within a supply chain. DRP enables the user to set certain inventory control parameters (like a safety stock) and calculate the time-phased inventory requirements. Its approach based on the bursting of the "BOM" of the distribution system is similar to an MRP which uses the "BOM" of product. Vollmann et al. position the DRP close to the marketplace and presents that "DRP is a link between the marketplace, demand management and MPS". The authors indicate the role of DRP is "to provide the necessary date for matching customer demand with the supply of products at various stages in the physical distribution system and products being produced by manufacturing."

The limit of DRP is caused by the fact that horizontal integration is necessarily limited to a few entities in the supply chain. Indeed, in the case of short-term customer demands, the upstream needs will

- either generate needs prior to the current date (cases of desynchronization and thus cases of out of stock)

- or can be absorbed by one or more stocks upstream of the chain (where the order is fulfilled).

Crama et al. (2001) recall the limits of this system which sometimes are caused by a desynchronization of production plans. Thus, the application of a DRP at the lower level of tactical planning only remains for short-term use. It is indeed necessary to already ensure a horizontal synchronization at the S\&OP level in order to predict a load/capacity adequacy on all the sites as well as their synchronization.

The synchronization is carried out by considering the material need and the components generated by the product BOM. It brings a much more complex tactical planning problem, which consists not only in generating production plans for the entities but in ensuring their synchronization. These models are therefore called multi-level. Synchronization will then be ensured by considering the balance of stocks that bind each entity in the chain.

As mentioned above, the understanding of multi-level planning problems can be achieved by assuming two approaches:

- A centralized approach 


\section{- A decentralized approach}

The centralized approach assumes that whatever the nature of the links between the component entities in the supply chain, it is possible to consider a master supply chain manager (or mediator) that has all the information and power to generate a synchronized planning of the supply chain. Certainly, this assumption assumes that there is a full collaboration between all the entities in the supply chain. This is why this hypothesis should be set rather for the case considering only the entities of a company than the cases including an external partner of the supply chain.

However, the literature mainly assumes that the centralized approach is not enough realistic. Therefore, numerous researchers suppose a decentralized approach where the actors in the supply chain share a certain amount of information but generate their planning independently. Féniès (2006) proposes to synthesize the processes of tactical planning by adopting the work of Simatupang \& Sridharan (2002) based on the notion of Collaborative Planning Forecasting and Replenishment. A lot of work has compared these two approaches. Thierry et al. (1994) demonstrates, on a case study, the interest of centralizing the decision to obtain an optimal solution. The centralized approach provides better solutions than those obtained through a decentralized approach. These conclusions lead many authors to study the relevance of information sharing at the heart of supply chains in order to improve their performance.

A vast literature has addressed the tactical planning problems. However, mosts of the works have focused on the mathematical model development of these problems and the elaboration of methods dedicated to these models which are called "Lot Sizing Problems". They are dedicated to the development of the different production plans (S\&OP, MPS and MRP).

\section{Mathematical model families of Lot-sizing problem}

Kuik et al. (1994) defined the lot-sizing problem as "the clustering of items for transportation or manufacturing processing at the same time". This problem arises whenever we need to decide the timing of productions as well as the quantity to produce each time. On one hand, the setup times or setup costs generated by these productions should be taken into account that may involve many different operations such as cleaning, preheating, machine adjustments, calibration, inspection, test runs or change in tooling, etc. Setup costs or changeover costs can be caused by a additional workforce needed to prepare the equipment or by the consumption of resources during the setup operations. Overall, in order to reduce the times of launching setups for efficiently utilizing the production resources, the production lot size should be reasonably large. On the other hand, these productions may generate products that need to be held in the inventory and deliver in a later period because the production may not be exactly synchronized with the received demand for a cost optimization objective. Inventory holding costs is thus incurred for tied up capital, product value depreciation and storing cost (warehousing, handling, shrinkage and insurance...).

The classic objective of lot-sizing problem is to determine an optimal/good production program that minimizes the sum of the setup costs and the inventory holding costs generated by this program. Essentially, an optimal/good trade-off between setup costs and inventory holding costs is what we need to reach for this objective and for satisfying customer demand. Consequently, finding this tradeoff is critical to improve the production performance that strongly influences the competitiveness of the company in the modern global market.

Since lot-sizing is always being recognized as a difficult optimization problem, considerable efforts on this problematic can be found in the lot-sizing literature for over a century. Different problematics are dedicated for problems in different contexts of the supply chain such as mono-site, multi-site or the entire supply chain. The criteria considered the most discriminating are presented as follows which are indeed not exhaustive, but provides a general overview of the classification: models and its extensions or characteristics such as:

- Nature of data (deterministic or stochastic), 
- Type of supply chain structure (single- or multi-site (plant)),

- Model of demand (constant or variable),

- Type of bill of material (single- or multi-level),

- Capacity constraints (with or without),

- Length of production periods (small or big bucket), etc.

First of all, even though most of the lot-sizing literature focuses on problems dealing with deterministic demand, the data considered here can also be stochastic for more realistic cases. In practice, production planning decision are very often made with forecasts in which errors may be hidden. This fact could strongly affect the solution procedure to apply. For similar reason, other parameters considered in this problem may also be stochastic, as surveyed in Brahimi et al. (2017).

Secondly, both single- and multi-site problematics appear in the lot-sizing literature. Obviously, the multi-site model is established for supply chain tactical planning in a more practical way. The replenishment and distribution problem between plants are also sometimes taken into account.

Thirdly, the type of deterministic demand in a lot-sizing problem can be further distinguished by the demand rate. Constant demand is evenly distributed over the horizon. Otherwise, it is called variable. Even though the objectives of these models are both to minimize production cost and to meet customers' demand, the fundamental difference of these models are in their purpose:

- Constant demand models aim at determining an optimal production cycle that is reproducible over the horizon. For example, the EOQ problem.

- Variable demand models determine optimal quantities to produce in all predefined periods of the discrete-time planning horizon.

Fourthly, the way in which the bill of material (BOM) is taken into account: the tactical planning problems consist of not only the planning for finished products but also for their components. These can be treated in two different ways:

- single-level: following the logic of MRP, the planning is executed level by level. It consists of computing the requirement of components from the finished products (called external demand) which are found at top of the BOM. This thus defines a level-by-level planning. It is therefore possible to use single-level models with which the quantity determined at the higher level of the BOM generates the (internal) demand at the current level. The limit of this approach is that there is no guarantee to provide feasible plannings due to the lack of consideration of the production capacity in the planning process.

- multi-level: multi-level models link the BOM levels by introducing the structure of BOM and expressing the internal and external demand. Such models ensure the feasibility of the solution. They also allow us to consider lead time between the levels.

Fifthly, capacity constraints are also an important aspect to be considered. Considering a capacity constraint significantly increases the computation complexity for determining an optimal solution, even for the most simple case.

Lastly, for variable demand models, lot-sizing models can also be distinguished based on their size of the production period, i.e., bucket. Both "small bucket" and "big bucket" models exists in the literature. On the one hand, for "small bucket" models, we assume that only one product can be produced during a micro period which can be short as hours. They aim at determining both production quantities and sequence of production. Obviously, the horizon length is reduced compared to the one in "big bucket" model. On the other hand, "big bucket" models are thus used to deal with problems with larger length horizon. In this case, several products can be planned in a same period, called a macro period, e.g. week or month.

In Figure 2.5, the classification of deterministic models of lot-sizing problems presented in Comelli et al. (2008) is given. In the single-level context, the main models are:

- Capacitated Lot-Sizing Problem (CLSP): A multi-item problem for satisfying dynamic demand with capacity constraints. This big bucket model is proven as a NP-Hard problem in Bitran \& Yanasse (1982); 


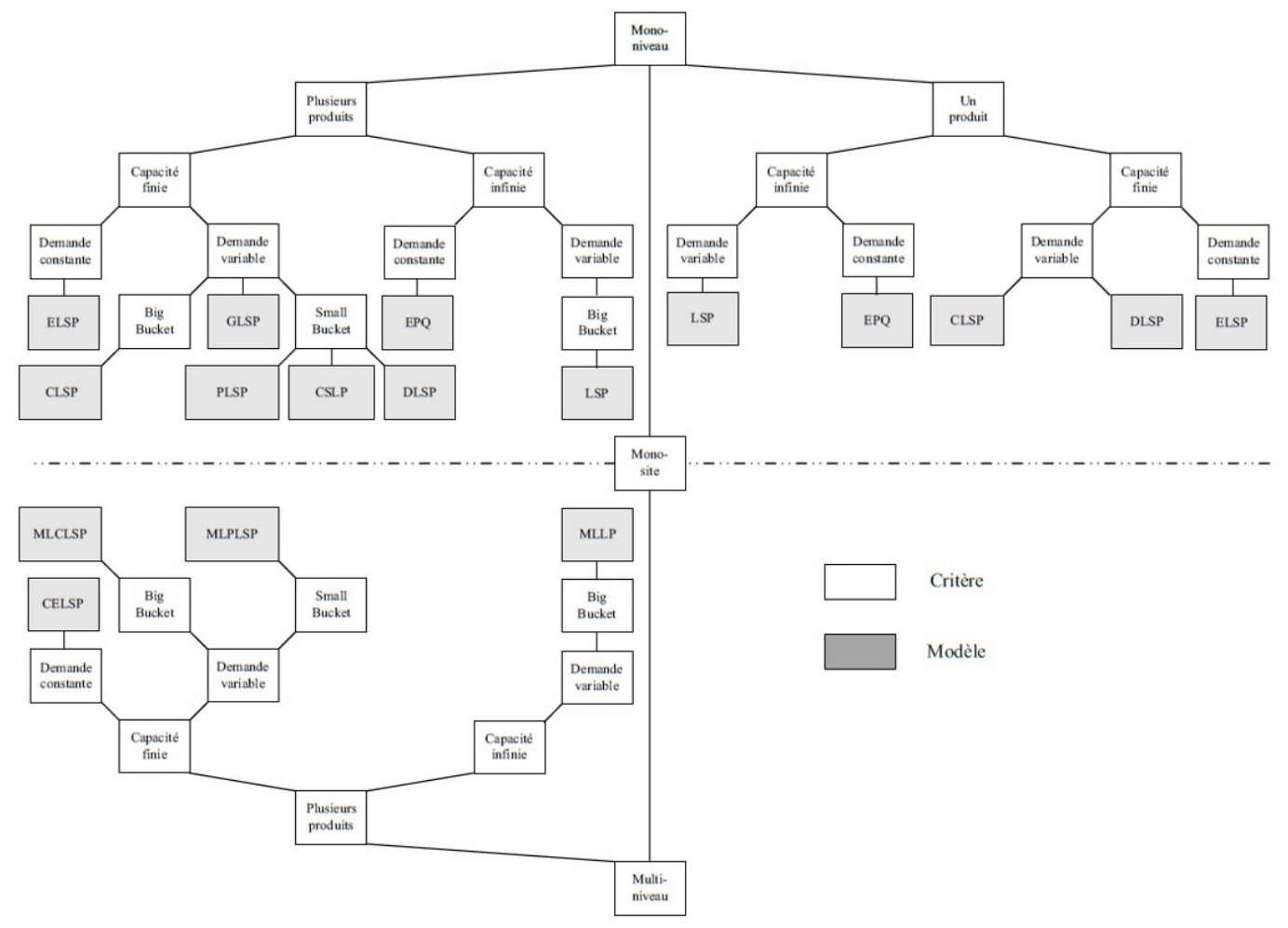

Figure 2.5 - Classification of lot-sizing models (Comelli et al. (2008))

- Lot Sizing Problem (LSP): when capacity constraints are not taken into account, the CLSP becomes polynomial (Wagner \& Whitin (1958)). In this thesis, we adopt the name used in Pochet \& Wolsey (2006), which is ULS for "Uncapacitated Lot-Sizing Problem";

- Economic Order Quantity (EOQ), Economic Lot Scheduling Problem (ELSP): The EOQ and the ELSP are single-item problems with constant demand. The time is continuous (not divided in the periods) and the planning horizon is infinite. The EOQ does not assume capacity constraints which make it polynomial thanks to Wilson's formulas contrary to the ELSP which is NP-Hard;

- Discrete Lot Sizing Problem (DLSP): The NP-hard problem DLSP is a small bucket model. It assumes that only one item may be produced by period, using the full capacity of the system. This is called the "all or nothing" assumption;

- Continuous Setup Lot sizing Problem (CSLP): In this small bucket model, contrary to the DLSP, The "all or nothing" assumption does not exist anymore. Thus, the capacity of periods may be not fully used;

- Proportional Lot Sizing Problem (PLSP): the main idea of the PLSP is to use this remaining capacity left by the CSLP model for scheduling a second item in the particular period;

- General Lot sizing and Scheduling Problem (GLSP): this one integrates lot sizing and scheduling of several products on a single capacitated machine. Continuous lot sizes are determined and scheduled. By this way, this model generalizes models using restricted time structures.

About multi-level context, models with similar hypothesis are considered. Therefore, their names are quite similar to the single level ones (with adjunction on ML prefix).

More classification is referred to the Lang (2010) and Brahimi et al. (2017).

The extensions of the standard lot-sizing problem include backlogging, perishable inventory, lost sales, time windows, multi-facilities, inventory capacity, setup carry over and remanufacturing, etc, which are not be considered in this thesis. The interested reader can refer to Brahimi et al. (2017) for further details.

In this thesis, we only consider three models without a capacity constraint. Using the aforemen- 
tioned classification, the three models are respectively:

- single-product, single-level and for constant demand that is same case as in the EOQ model;

- single-product, single-level, big bucket and for variable demand that is same case as in the $U L S$ model;

- multi-level, big bucket and for variable demand that is same case as in the MLLP model;

\subsection{Financial supply chain}

\subsubsection{Introduction to financial supply chain}

As defined by Dalmia (2008), the supply chain can be categorized into the physical and the financial supply chain. The former consists of processes involved in the physical movement of goods, e.g. inventory management. The latter includes the movements of funds resulting from the physical supply chain, (see Figure 2.6. Supply chain management and the optimization of physical flows have received more attention than optimizing financial flows between the different supply chain partners. However, this situation seems to be evolving with the advent of the Financial Supply Chain (FSC). The term Financial Supply Chain Management mirrors the concept of Supply Chain Management. It recognizes that there is a chain of dependent events that has an impact on the working capital of an organization. On the buy-side, the timing of purchases, inventory, payment terms with suppliers and discount arrangements all impact working capital. The management of that chain of events (in so far as finance can influence them) is part of Financial Supply Chain Management (FSCM). More and more firms engage in advanced methods of managing financial flows along their supply chains (see Figure 2.7). FSCM thus requires the internal coordination between financial managers and supply chain managers of the company, as well as the external collaboration with service providers (e.g. banks), suppliers, and customers. Traditionally, financial flow management focuses on optimizing a single firm's cash flow. However, FSCM extends the scope to the entire supply chain (see Wuttke et al. (2013)). The authors provide a summary of recent FSCM methods which involves many diverse fundamental elements of FSCM:

- Buyer credit: Term financing provided to finance suppliers (e.g. advance payments or deposits) ( Chauffour \& Farole (2009); Thangam (2012))

- Inventory/work-in-progress financing: Buyer provides loan to supplier to finance work-inprogress (Chauffour \& Farole (2009))

- Reverse factoring: The supplier obtains a credit from a bank and pays the interest according to the buyer's interest rate. The buyer pays the loan principal to payment terms (Tanrisever et al. (2012); Klapper (2006))

- Supply chain finance: An automated solution that enables buying firms to use reverse factoring with their entire supplier base, often providing flexibility and transparency of the payment process ( Shang et al. (2009); Demica (2007))

- Electronic platforms: Systems offered by third parties to electronically connect trading partners with financial institutions to automate payment processes (Sadlovska (2007))

- Letters of credit: A financial institution provides guarantee to exporters by replacing the importer's risk with its own default risk (Amiti \& Weinstein (2011))

- Open account credit: A buying firm receives credit from suppliers without formally offering securities or involving third-party security (Malouche \& Chauffour (2011); Chauffour \& Farole (2009))

- Bank loan for financing the supply chain: Short-term or medium-term financing provided from a bank involving working capital and pre-export finance (Chauffour \& Farole (2009))

Due to its high practical relevance, analytical research has recently shown significant improvement potential of inventory models if financial flows are taken into consideration(e.g. Babich \& Sobel (2004); Gupta \& Wang (2009)). 


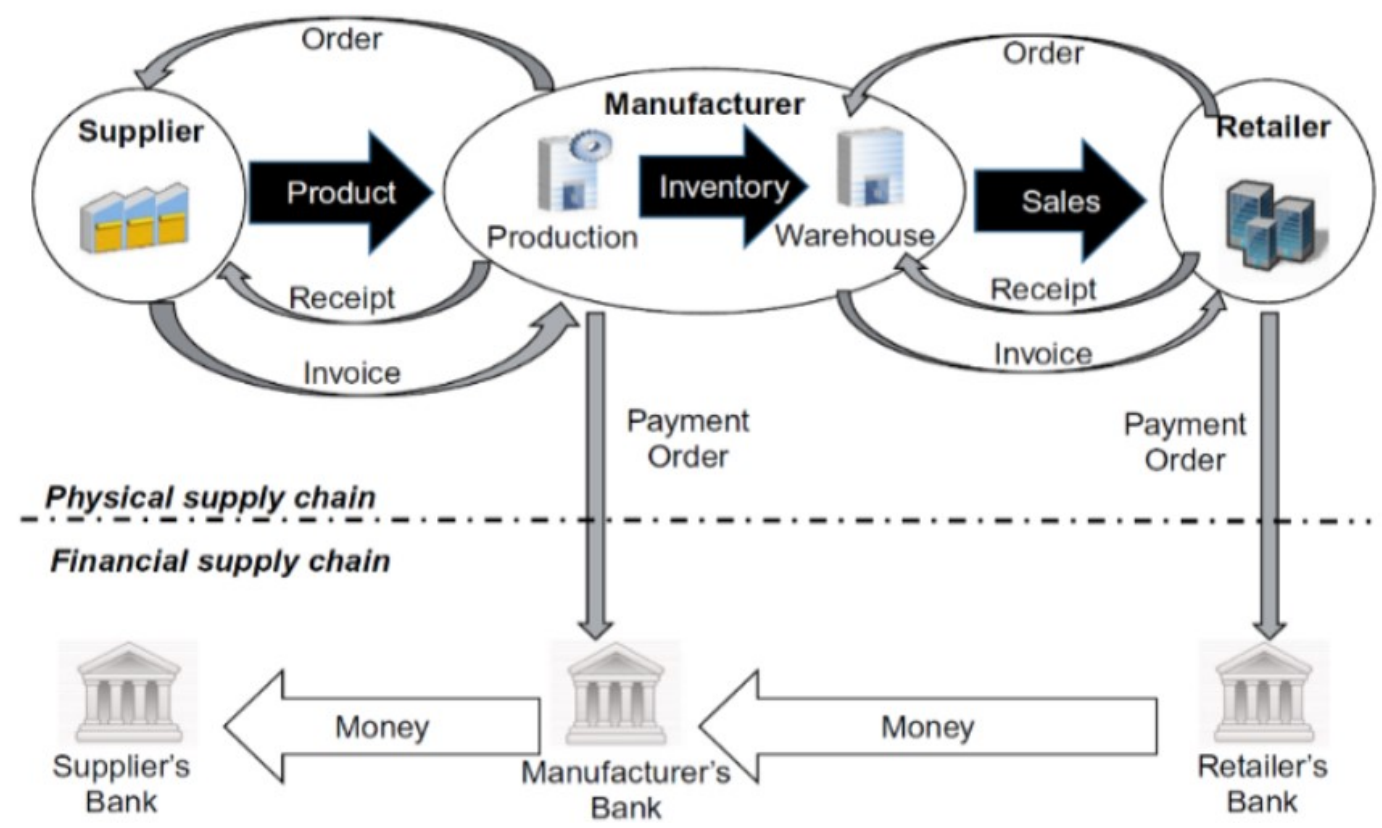

Figure 2.6 - Physical and financial supply chain

\section{Financial status of company}

In accounting, three documents are established to measure the company's financial statues, i.e. financial asset position, financial performance and cash flows level. They are :

- Profit \& loss (P\&L) statement: a financial statement that summarizes the revenues, costs and expenses incurred during a specific period of time, usually a fiscal quarter or year. These records provide information about a company's ability or lack to generate profit by increasing revenue, reducing costs, or both. The P\&L statement is also referred to as "statement of profit and loss", "income statement," "statement of operations," "statement of financial results," and "income and expense statement." A key concept of the P\&L statement is the gross operating surplus (GOS), which represents the surplus created by the operation of the company after remuneration for the labor input factor and the taxes linked to production. The GOS is measured before the depreciation decisions and financial charges arising from the company's financing choices. It appears to be the balance between operating revenues that have resulted or will result in a cash inflow and, on the other hand, operating expenses that are disbursed or are expected to be disbursed. This is a potential monetary surplus and therefore allows to measure the capacity of the company to generate cash resources from its exploitation. In this sense, the GOS is both an operating balance and a measure of gross cash flow.

- Balance sheet: a financial statement that summarizes a company's assets, liabilities and shareholders' equity at a specific point in time. These three balance sheet segments give investors an idea as to what the company owns and owes, as well as the amount invested by shareholders. The balance sheet is built based on the main functions of the company: investment (which represents stable resources), financing (which comes from stable resources) and operating (which translates into liabilities and short-term assets: Inventories, trade receivables, trade payables). Cash flows arising from operating activities are reflected in the balance sheet in the form of receivables or debts. The outcome of these three cycles results in an impact on cash flow with either a surplus or an insufficiency.

- Cash-flow statement: The cash-flow statement (CFS) is a mandatory part of a company's 


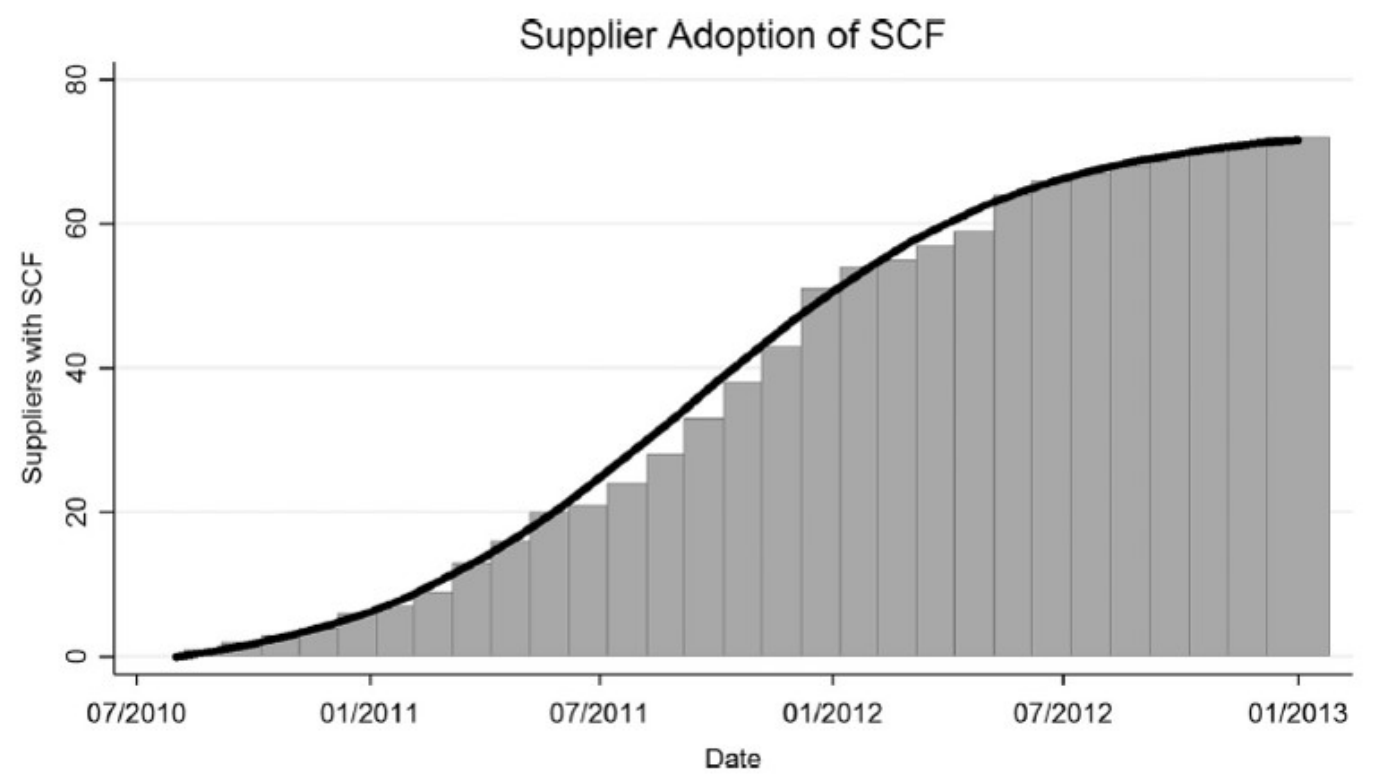

Figure 2.7 - Increasing number of firms using supply chain finance method in Germany (Wuttke et al. (2016))

financial reports since 1987 - records the amount of cash and cash equivalents entering and leaving a company. The CFS allows investors to understand how a company's operations are running, where its money is coming from, and how it is being spent. Treasury is a leading indicator for financial management and analysis. This statement provides the evaluation of companies on their ability to generate liquidity and meet their commitments. It has the advantage of providing an objective indicator, cash, which is commonly used in the company valuation. Cash is also fundamental to evaluate the failure risk rate.

\subsubsection{Working capital requirement: link between physical and financial flows}

"Cash is king" - despite the fact that the cash has its own costs. Cash is the most liquid asset and is presented commonly on the balance sheet as the first item. Management of cash is of great importance for a company. If adequate cash is not available when it is needed, the situation lead to bankruptcy. Management of cash and liquidity involves providing sufficient funds to the business for meeting various requirements at the right time, such as repayment of bank loans, payment of taxes, payment of wages, purchases of raw materials and inventory etc. Moreover, holding the cash entails a precautionary motive in order to meet unforeseen events. Therefore, the cash must be managed properly and provided for arising contingencies. In the following section, we present the current economic and financial context. Next, we explain the direct link between the cash and working capital requirement(WCR) which is chosen as the financial aspect considered in tactical planning. Then, we describe the main concept of the WCR and how it links the physical and financial flows.

\section{Economic context}

Over the past few years, the succession of economic and financial crises has constantly jeopardized the competitiveness and even the life of companies. The Credit Crunch, in other words, the slowdown in credit lending by financial institutions, is one of the main causes of this situation. According to a recent study of the Organization for Economic Cooperation and Development (OECD), 115,813 companies, mostly small and medium-sized enterprises (SMEs), have closed down in France in 2009 and 2010. The number of these bankruptcies is increasing in 2012. According to Coface and the 
Altarès consulting, the forecast of bankruptcies in the crisis of 2012 arises to 63,500 and of job saving plan (plans sociaux) is more than 317,000. At the same time, financing constraints which are reinforced by the difficulties of the banking sector and the new regulations strongly penalize growth (10 to $15 \%$ of turnover loss in the industrial sector) and operational performance (20 to $25 \%$ loss in productivity) for all involved actors (suppliers, subcontractors, payers).

Especially, in early 2012, potential instabilities in France, Greece, and in other European countries after the elections have been considered as the cause that may further worsen the economic and financial situation in Europe in the Euro debt crisis period. The major European banks were feared to be heavily stroked and began hoarding cash in large quantities since the end of 2011. Until the end of March 2012, the top 10 European banks deposit cash at the central bank of different countries around the world totaled nearly 1.2 trillion US dollars, an increase of 128 billion dollars compared with the amount at the end of the fourth quarter 2011, equivalent to a $12 \%$ increase. If we compare it with the end of 2010, the amount increases $66 \%$. Therefore, the top 10 European banks no longer lend money to customers, or for other purposes, in order to ensure a large number stored into the central bank, as their self-protection and self-help. If the expansion of the European debt crisis leads to a worse credit crunch, financing will become more difficult. Moreover, if their credit levels were downgraded, it may promote a large number of divestment of their clients. Storing sufficient cash allows them ensure the source of funds for all emergency events. The top 10 banks kept 440 billion dollar in total in the central banks from the end of September 2011 to the end of March 2012, most of which came from the recent three-year low-profit refinancing operations launched by the European Central Bank. The measure was intended to quell the liquidity crisis of the financial system caused by the European debt crisis and hopes to drive banks to expand lending and to buy government bonds and then thus alleviate European economic and financial problems. The top 10 European banks includes Banco Santander, BBVA, Deutsche Bank, UBS, Credit Suisse, BNP Paribas, Societe Generale, Barclays, Lloyds Banking and Royal Bank of Scotland Group.

The liquidity and financing needs of the operating cycle (purchasing, production and inventories) of SME/SMIs have become the fatal weakness of the "Supply Chain" and the competitiveness of industrial sectors. Paradoxically, companies are demanding billions of aid from banks and public authorities to finance their Working Capital Requirement (WCR), even though almost one-third of these needs result from a lack of collaboration between customers and suppliers, and the weak synchronization between operational flows and financial flows. An Ernst \& Young study on the WCR performance indicates almost 100 billion euros in excess needs among a panel of 130 French companies in 2010 .

\section{Definition of Working capital and Working capital requirement}

Coordination between physical flows and financial flows is essential to ensure economic profitability, customer satisfaction and to ensure the sustainability of the company. In practice, both academics and practitioners agree that the consolidation of the physical and financial supply chain enhance the cash flow predictability, reduce risk-related costs, improve working capital and cash flow level as presented in Zeballos et al. (2013). However, it can be very difficult to achieve this transversal subject concerning specific objectives which may be in conflict with each other. Decisions of operation are made from an operational point of view considering inventory, service levels and capacities which drive the results on profit, working capital requirement (WCR) and return on investment (ROI).

In this thesis, we adopt the francophone expressions of working capital and working capital requirement :

- Working capital: Corresponds to the difference between permanent capital and immobilized assets. It allows a company to verify if the long-term resources are able to cover the immobilized assets. When positive, it is a surplus of resources to finance part of the company's short-term business. Negative, it can reveal a detrimental financial imbalance, especially if 
the company is in the growth phase.

- Working capital requirement: The measurement of the financial resources that an enterprise must use to cover the financial need arising from cash flow mismatch. This mismatch is between the disbursements (expenses and operating revenues required for production) and cash receipts (selling goods and services) which are linked to its operating activity.

\section{The concept of Working capital requirements}

The WCR has two components: operating working capital requirements (OWCR) and nonoperating working capital requirements (NOWCR). The NOWCR is generated by the time mismatch of cash flows which are related to non-operating operations (debt on investment, dividends to be collected or to be disbursed, exceptional events ...). Given its marginal importance, the NOWCR has no particular economic significance. Furthermore, the NOWCR is difficult to predict and analyze and sometime event-based, contrary to the OWCR which is recurrent. Therefore, we only consider OWCR in this thesis which will be referred as WCR.

The operating cycle corresponds to the regular and recurring activity of the company. This regularity entails permanent financial consequences because they are commonly renewed. Consideration of this dynamic in financial analysis is ensured by the notion of financing need for operating or working capital requirements. The WCR is thus analyzed as a financial need linked to the company's activities which requires a financial resource to cover.

The WCR finds its justification in very simple principles:

- A receivable (or payable), although acquired and certain, is not usually paid immediately by the customer (to the supplier);

- A stock is not sold immediately and products remain in stock for a period before being sold.

These different gaps will generate a financial need for the company, which will have to be financed either by the settlement period negotiated with the suppliers, by the working capital or by the treasury. Consequently, the WCR can be expressed by the following formula :

$$
\mathrm{WCR}=\text { Account receivable }+ \text { Inventory value - Account payable }
$$

The operating WCR is characteristic of the sector according to their activities pattern. However, the WCR is usually positive. The concept of WCR thus highlights the fact that companies sometime do not finance all their operating jobs (stock requirements and customer loans) with resources coming from the operating cycle but by additional funds from working capital or free cash flow. The free cash flow is calculated as the difference between the working capital and the working capital requirement. It represents the cash remaining after financing the operating cycle of the company. Therefore, the relation between these elements is formulated as follows:

\section{Working capital $=$ Working capital requirement + Free cash flow}

Thus, the lower the working capital requirement, the greater the cash position, which shows a strong relation and a solid financial structure between these two terms.

The WCR results from the existence of the time mismatch between the operating flows (expenses / income) and cash flows (receipts / disbursements). These differences are of different origins:

- They first originate from the production process itself, where exists a production lead-time which already separates the exit of raw material from inventory and the entry of finished product into inventory;

- Second, the storage duration of finished products represents the time of immobilizing the product value before its sale;

- Third, the delay in payment from the client settled by negotiation which delays the payment collection; 
- Finally, the company benefits from the payment delay granted by its own suppliers, which is considered as a financial resource.

Generally, firms with negative WCR and firms with a positive WCR can be distinguished:

- Positive WCR:

The positive WCR is characteristic of general industrial firms whose operating cycle includes a major production phase. The operating cycle of an industrial enterprise includes purchasing the raw material, intermediate and finished product production, storage of these items, and selling the finished product. For example, the suppliers of raw materials grant an average credit of 6 weeks, the manufacturing costs are settled on average 3 weeks after their commitment. The company gives its customers a settlement period of around 4 weeks, which means that companies pay almost all the expenses incurred in the production process before the products are sold.

The WCR is thus equal to the financing requirement of the operating activities plus the need due to the credits granted to the customers and less the resources coming from the credits granted by the suppliers.

- Negative WCR:

A negative WCR is characteristic of retail, hospitality and e-commerce sectors. In the case of large retailers, and hotel and restaurant customers, customers pay in advance or with a significantly small delay, supplier payment delays are high (on average 60 days in France) and inventory turnover is often rapid (around 15 days in the case of a prominent retailer). Similarly, the e-commerce sector (Amazon, other e-shops...), benefits from a negative WCR because customers pay for the product before the delivery which is deferred and sometime in a great delay of a month and the supplier delays are also significant.

This negative financing requirement therefore constitutes a financing resource. These shortterm resources are stable because they are due to the market structure and the operation of the sector and can therefore finance capital investments.

In some sectors, seasonality is also a factor to be taken into account. In highly seasonal sectors (e.g. textiles, coats and swim-wear production, ice-cream production, etc.), the working capital requirements may be very different between two periods. An aforementioned static analysis of the WCR is limited to an aggregated situation by the end of the considered period. It can therefore be very far from the economic and financial reality of the company. Thus, this type of business may experience an important demand at a time of year, which will result in a high turnover of stock. It is therefore necessary for the company to have a minimum security inventory level throughout the year, but it must also be able to predict these fluctuations so that it can finance the operating cycles with high production activity to avoid out-of-stock situations. In these cases, it is interesting to analyze the evolution of this indicator during the year.

\section{Working capital requirement: link between the physical and financial flows}

In practice, the WCR is known as a key indicator to monitor and control the financial situation of a company. The WCR is the minimum amount of financial resources to cover the logistic cost before receiving the clients' payment. As we mentioned in previous paragraphs, the level of cash flow is remaining cash after covering the WCR by using working capital. Optimizing the WCR generates additional cash flow that will allow efficiently allocating resources (for paying suppliers, employees... ) and to continue business development (for mergers and acquisitions, etc.). By this direct link with cash flow, the WCR increasingly draws attention of finance departments especially in economic crisis when funding is difficult to obtain. On the other hand, financial managers are always limited by operational performance concerning levels of desired financial performance . A trade-off is generally difficult to reach in practice since the outcome of a decision on both the financial and 
operational side is not always predictable. For example, improving the working capital position might lead to counterproductive outcomes: supplier prices will be increased due to an exaggerated decrease of accounts payable which may consequently increase the WCR. Thereby, complexities in both theoretical and implementational levels should be carefully considered.

In this thesis, we consider WCR as the link between the physical and financial flows by integrating the financial cost of the WCR in the classic lot-sizing that only minimizes the total logistic cost. The new models thus take into account the financial consequences involved by the operation decisions in order to obtain new optimal production plans. The main reason of combing the WCR financial cost and the logistic cost is that the annual average inventory value is involved in the accounting formula of the WCR. Fundamentally, the inventory level is the key factor we optimize with tactical planning model. However, in accounting, the WCR is calculated using information provided by the Profit \& loss (P\&L) statement and balance sheet. All this information only represents an aggregated situation by the end of the financial year. For this reason, we can not directly adopt the traditional calculation of the WCR and new generic models of the WCR are proposed in following chapters.

\subsection{Project "Risk, Credit and Supply Chain Management"}

This thesis is financed by the project "Risk, Credit and Supply chain Management" (RCSM). In an economic and financial context, the credit crisis has led to the emergence of new solidarity practices based on the pooling of excess cash between companies. For example, the Airbus group played the banker for some of its subcontractors who were in cash shortage. Airbus has mobilized 1 billion Euros for this purpose. In the same spirit, the shipbuilder STX France of Saint-Nazaire, with its 4,000 suppliers and subcontractors, proposes to consolidate and pool the financing of shipbuilding projects (projects based on operating cycles of 18-24 months and are very risky and very demanding in financing) for shipowners who refuse to pay any down payment and pay almost only on the delivery day. For this purpose, STX France has recruited specialized teams in financial engineering to facilitate the implementation of financing for these shipbuilding projects. Even if the actual cases are very few, such approaches to pool financial needs and excess cash motivate the establishment of the RCSM project.

Therefore, the RCSM project aims at developing a pooled and consolidated cash flow and working capital requirement financing platform within the supply chain for industrial sectors (see Figure 2.8).

The objectives are as follows:

- Reducing excess WCR (stocks and payment delays) at the sector level through better collaboration between clients, their networks of multi-ranked SME / SMI suppliers, and funding agencies;

- Free up financing capacities (and thus free up growth potential) for SMEs / SMIs, by pooling and smoothing the financial needs and cash surpluses between the actors in the supply chain;

- Reduce the financing cost and the cost of risks (operational, financial) for all supply chain actors, thereby improving the competitiveness of industrial sectors;

- Accelerate cash flow in the supply chain by synchronizing payment flows and physical flows across the supply chain and by optimizing the management of inter-company loans;

- Secure and improve the performance of the financial Supply Chain through risk pooling and supervision.

This project is fonded by both the industrial partner like Weave Consulting, Kyriba Corporation, People's Bank of the Alps, Marsh group, and academic institutions like IGR-IAE of Rennes, Lip 6, Armines (IMTA Nantes) and INSA of Rouen. 


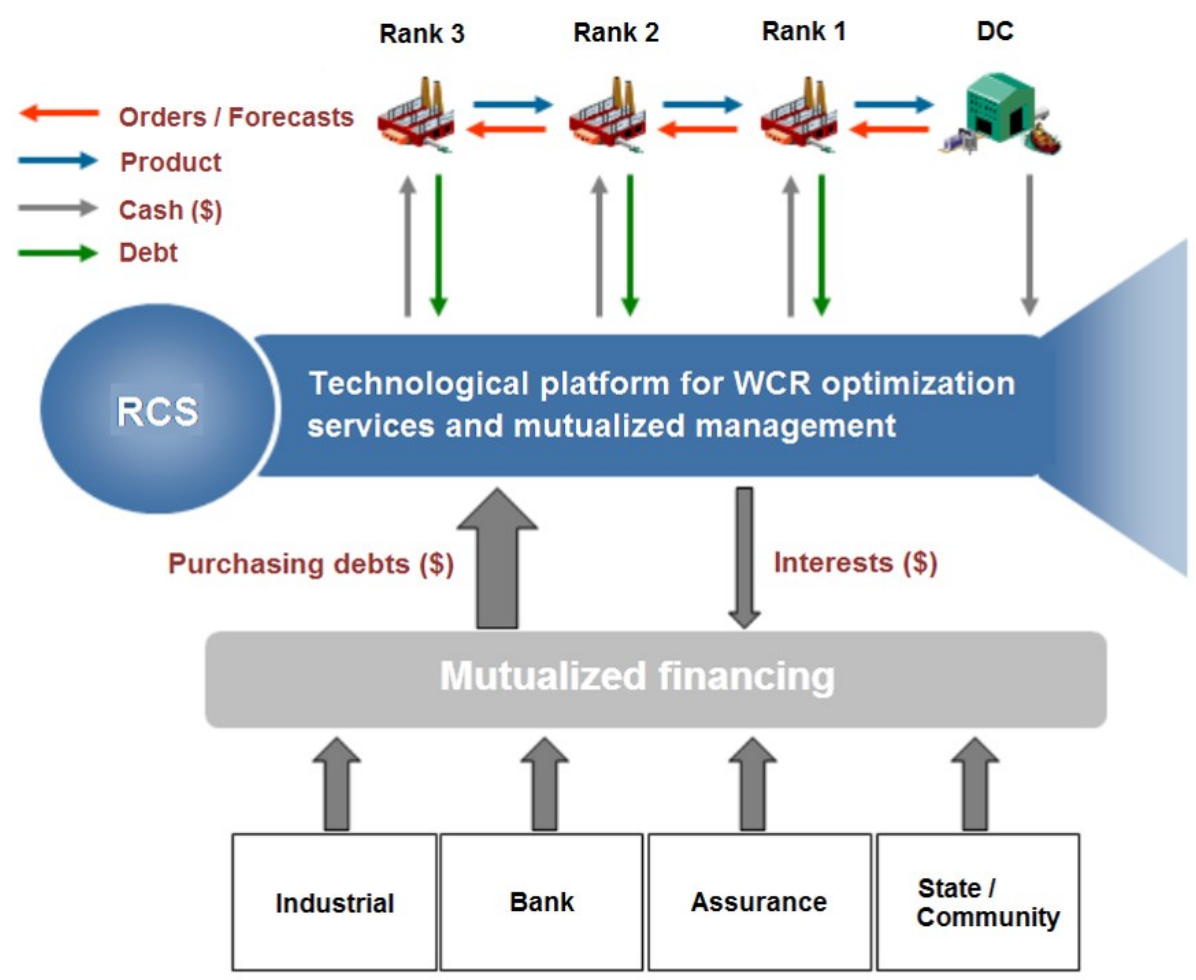

Figure 2.8 - Project RCSM

\subsection{Problematic and contribution}

This thesis is positioned in the main part of the RCSM project and its objective is to develop tactical planning models based on the classical lot-sizing models considering the financial cost of WCR. In other words, we consider not only the classic logistic costs (purchasing, setup, production and inventory costs), but also the financial cost to cover the production-related costs before the receipt of the customer's payment. This integration of the WCR cost reveals the financial consequences of the operation decisions that can not be directly derived from the classic lot-sizing models. In this thesis, we assume that the financial needs of the supply chain members are covered by one and only one financial resource (e.g., a bank or its own working capital). Thus, the associated portfolio problem as addressed in Serrano et al. (2017) is not considered in this thesis.

The main contributions of this thesis are summarized as follows:

- Chapter 2: Study of the constant demand case with infinite capacity.

- Proposition of WCR model (for this continuous time case);

- Integration of WCR financing cost in the classic Economic Order Quantity (EOQ) model;

- Formulation of optimal quantities in different cases with analytical solutions;

- Comparison with the classic model;

- Numerical illustrations.

- Chapter 3: Study of a single-level, time-varying demand case with infinite capacity

- Proposition of WCR modeling (for this discrete time case);

- Integration of the WCR financing cost in the classic Uncapacitated Lot-Sizing (ULS) model;

- Proof of the Zero-Inventory-Ordering (ZIO) property;

- Proposition of a polynomial algorithm to solve the problem;

- Comparison with the classic model and sensitivity analysis of the new parameters.

- Chapter 4: Study of a multi-level case with time-varying demand and infinite capacity

- Proposition of WCR modeling (for two-level "supplier-client" case); 
- Integration of the WCR financing cost in the classical two-Level Lot-sizing (2ULS) model;

- Proof of the Zero-Inventory-Ordering (ZIO) property;

- Proposition of a polynomial algorithm to solve the problem;

- Comparison with the classic model and sensitivity analysis of the new parameters;

- Generalization to multi-level case; 



\section{Integration of working capital requirement financing cost in EOQ model}

\subsection{Introduction}

At the beginning of our work, we are interested in problematics of single-level, single-product planning. In this chapter, we firstly provide a state of the art of financial aspects which have been integrated into the Economic-Ordering-Quantity (EOQ) model in the literature. This investigation shows that only a few studies have involved the working capital requirement in the past and absence of consideration of working capital requirement in the tactical production planning context. Secondly, we focus on establishing a suitable model of the working capital requirement (WCR) in single-level, single product, and for constant demand over continuous-time planning horizon case. It is further integrated into the classic EOQ model.

\subsection{The Economic Order Quantity model}

In daily business, operations managers deal with the question of quantities to produce or buy for satisfying external (e.g., customer demand) or internal needs of some products. This decision should be carefully made due to its significant consequences in both operational and financial aspects. As indicated by Schwarz (2008), the fundamental decision about the "order-quantity" is to identify "the circumstances under which the choice of the order-quantity makes a lot of overall differences" comparing with current policy. Since it is simple to implement the EOQ model which is able to provide an optimal ordering quantity with assumptions. It deserves a great deal of management attention and thoughtful judgments if a significant overall difference is revealed by the result of the EOQ model. For this reason, as the creator of the EOQ model Harris (1913) suggests that using the Wilson's formula "as a check for a trained judgment, is at least warranted".

\subsubsection{The EOQ model}

This model focuses on to a problem concerning management of a single-product production over an infinite horizon under the following hypotheses:

- the demand is constant and intervenes uniformly over time, when $d$ pieces being withdrawn from the stock at each unit of time; 
- at each production, a fixed cost, $K \geq 0$, must be paid, as well as a variable cost $a \cdot Q$ that is proportional to number of produced units, $Q(a \geq 0)$;

- production is immediately executed (which means that no lead time of production is considered);

- backlogging is not allowed;

- for each unit of time and each product in the stock, a constant unit cost of storage, $h \geq 0$, is to be paid (that is proportional to product value).

In the EOQ model, three types of costs, namely unit purchase cost, inventory holding cost and the fixed order costs, are taken into accounts. First, the unit purchase cost $a$, measured in dollars per unit, is assumed to be fixed and independent of the order (manufacturing) quantity. Although, the order quantity-related discounts exists in the literature. Second, the inventory-holding cost $h$ is considered in this model as a product value depreciation. It may be caused, for example, by the deterioration of products, holding costs for storage, taxes or insurance fees and time value of money during a holding period. $h$ is measured in dollars per (unit $\times$ time). In real world scenarios, the value of $h$ is approximately accounted between $25 \%$ and $50 \%$ of product value. Besides, it also depends on the company's profitability (i.e. cost of capital) and the product's risk of obsolescence. However, determining the exact value of this cost is generally difficult for numerous aspects that are to be considered, which may also vary over time. Third, the fixed order cost is denoted as $s$ and measured in dollars in the EOQ model. Therefore, it is independent of the order quantity and represents all the costs for placing an order excluding the cost of units themselves. For example, $s$ may include any administrative cost of placing and/or receiving an order (e.g., paperwork and inspection); or, in a production scenario, it represents the cost of machine setup for the production of demand. In practice, fixed order costs are always very significant costs. As stated in Schwarz (2008), "a set-up (i.e., equipment changeover) in pharmaceutical manufacturing, for example, can shut down production and engage dozens of workers for weeks at a time; thereby costing thousands of dollars." For the similar reason for $h$, determining the exact dollar value of $s$ is also challenging.

The objective of the EOQ model is to determine the optimal ordering quantity, $Q^{*}$, that minimizes the sum of up-mentioned costs in the function of $Q$ over the considered horizon. The result represents an ordering/production cycle that repeats over the infinite time horizon. The overall cost is denoted as $C(Q)$ that is in function of $Q$. Mathematically, this total cost includes average unit cost, average inventory holding cost and average fixed ordering cost, as written as follows:

$$
C(Q)=a \times D+h \times \frac{Q}{2}+s \times \frac{D}{Q}
$$

Detailed explanation of this equation is presented in Schwarz (2008) for each term.

Trade-off between costs: Since the unit cost is independent of the order quantity and other terms vary inversely following the variation of $Q$, we essentially look for a trade-off between the inventory holding cost and the fixed order cost, as shown in Figure 3.1. This trade-off that minimizes the sum of these costs is obtained when the derivative of (3.1) is equal to zero:

$$
\frac{d C(Q)}{d Q}=\frac{h}{2}-\frac{s D}{Q^{2}}=0
$$

Therefore, the optimal ordering quantity, $Q^{*}$, is obtained when $\frac{h}{2}=\frac{s D}{Q^{2}}$. Named as Wilson's formula, $Q^{*}$ can thus be expressed as follows:

$$
Q^{*}=\sqrt{\frac{2 D s}{h}}
$$

A formal sensitivity analysis can be found in Lowe \& Schwarz (1983) for different management scenarios. 


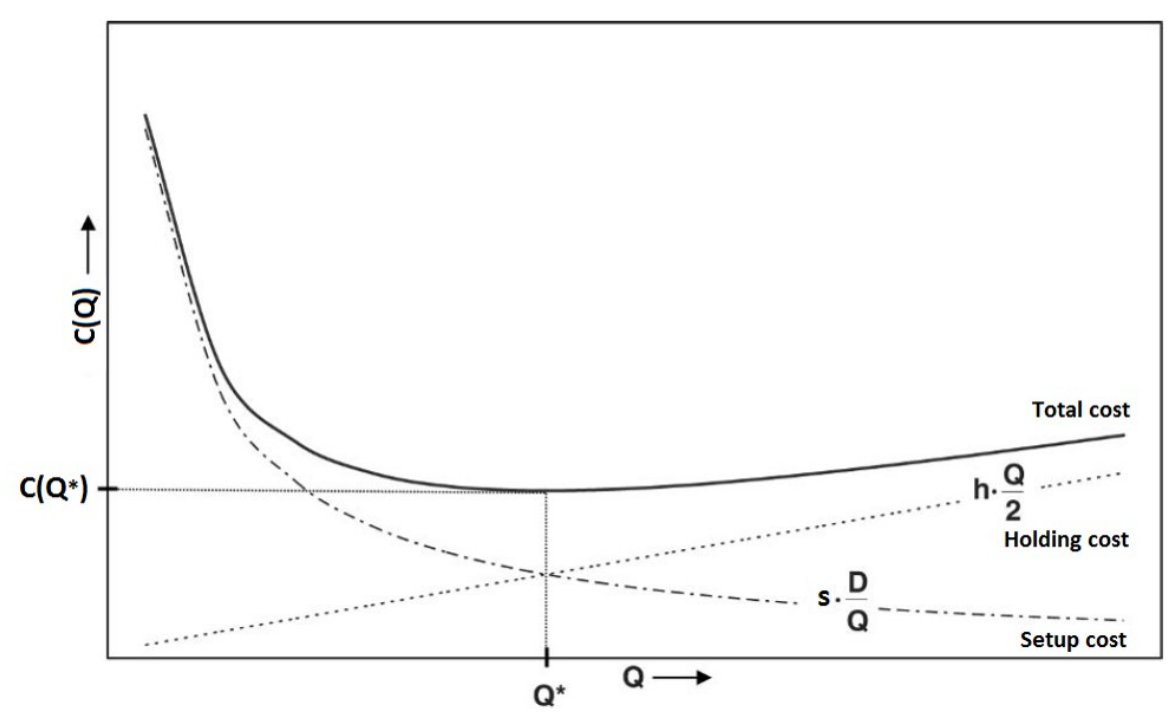

Figure 3.1 - Illustration of trade-off between costs in basic EOQ model

Classification: During a century of evolution from Harris's basic EOQ model, this problem has continuously attracted researchers' attention. In the review of Andriolo et al. (2014), the authors analyze 219 papers selected from 1913 to 2012 which can be immediately classified into three major sub-systems by the type of input data: deterministic, stochastic and fuzzy models. In Figure 3.2, Andriolo et al. (2014) illustrate the historical evolution of the EOQ model according to the chronological appearance of new aspects and assumptions being considered based on the reviewed papers. Then, the authors further propose a detailed classification of these three types of models based on their extensions. For instance, the extensions of the deterministic model exist for integrating aspects of time-varying demand, backlogging, quantity discounts, trade credit, deteriorating items, imperfect quality items, inflation effect, remanufacturing, finite production rate, sustainability into the classic EOQ model. Some other extensions of the classic EOQ model are discussed in Schwarz (2008).

This thesis focuses on the integrating financial aspects in tactical production planning problem. In the following paragraphs, we thus only discuss the financial aspects considered in the literature of EOQ problems.

\subsubsection{Financial aspects considered in the literature}

As shown in the classification of Andriolo et al. (2014) and after reviewing other articles in inventory management literature, it is indicated that four financial aspects are mainly considered:

- Trade credit;

- Time value of money;

- Inflation;

- Cost of capital.

\section{Trade credit}

Trade credit is an agreement that is determined between two companies, where a customer can delay payment in cash to the supplier after receiving purchased goods. In practice, trade credit is usually given for a fixed number of days, such as 30,60 or 90 days. Its main purpose is to stimulate the firm's sales and it is considered as a financial effort to strengthen the relationship with its customers. It is commonly used as a source of short-term financing for the purchase of goods and services. Essentially, trade credit terms offer firms contractual solutions to informational asymmetries between buyers and sellers. The credit period allows buyers to reduce uncertainties 


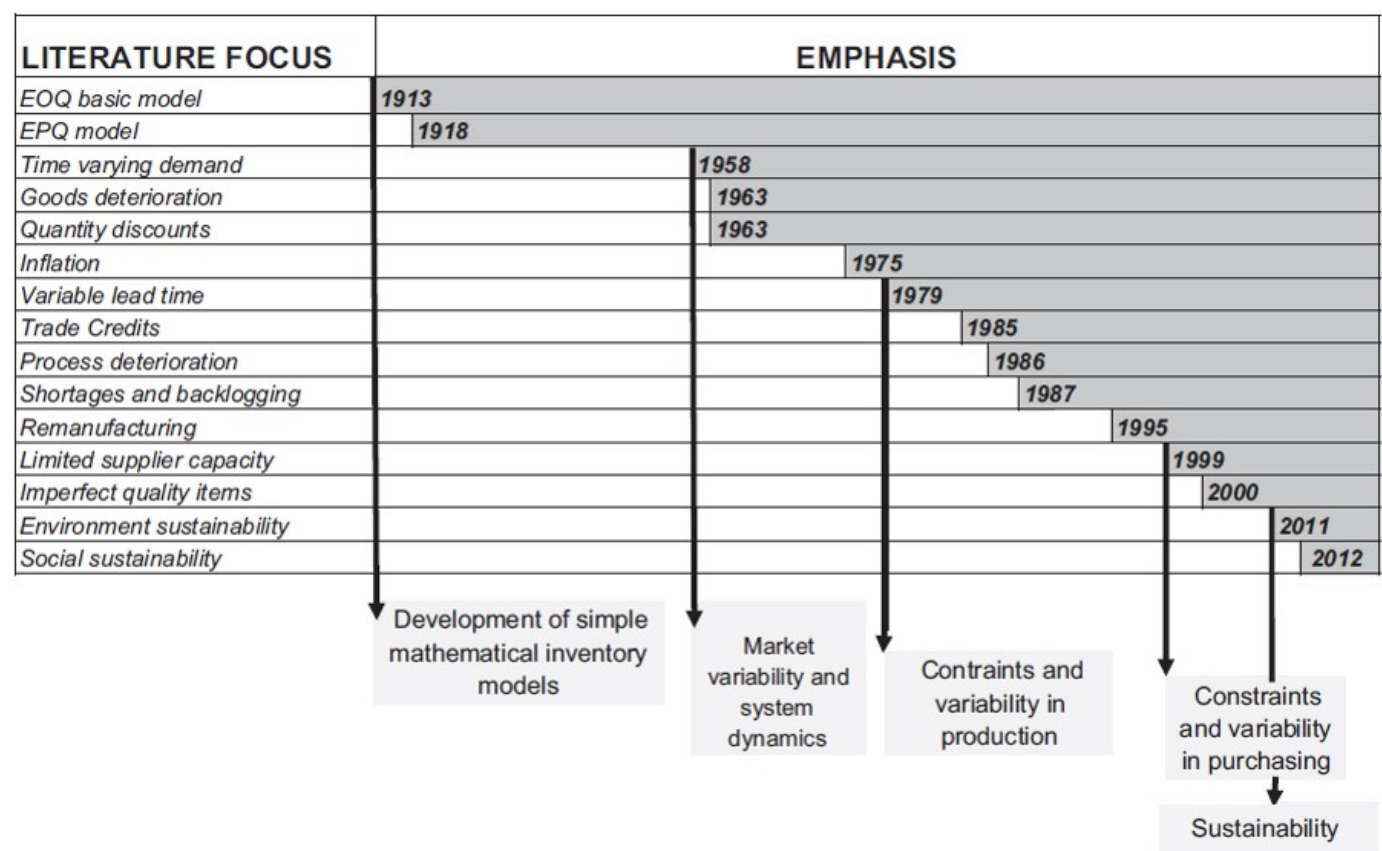

Figure 3.2 - Historical evolution in relation to the aspects and assumptions considered in EOQ literature (Andriolo et al. (2014))

concerning product quality prior to payment, while the seller can reduce uncertainties concerning buyers' payment intentions by prescribing payment before/on delivery or through two-part payment terms and other mechanisms. Variation in trade credit terms also offers firms price-discriminating opportunities.

The first basic work on this topic is conducted by Goyal (1985). In his model, two cases are discussed:

- Case 1: The replenishment period (the stime interval between successive orders) $\tau=\frac{D}{Q}$ exceeds the trade credit period $t(\tau \geq t)$. In this case, the customer has to pay an interest charge for items kept in stock, for the time that exceeds the trade credit period. The similar notation is adopted as in aforementioned EOQ model with $C$ referring to total annual costs, $\alpha_{c}$ is added as interest charges per dollar of investment in stocks per year and $\alpha_{d}$ as interest that can be earned per dollar per year. The annual total cost plus the charged interest minus the earned interest is formulated as follows:

$$
C(\tau)=\frac{s}{\tau}+\frac{h D \tau}{2}+\frac{a D \tau \alpha_{c}}{2}+\frac{a D t^{2} \alpha_{c}}{2 \tau}-a D t \alpha_{c}-\frac{a D t^{2} \alpha_{d}}{2 \tau}
$$

By deriving equation (3.4), the economic order quantity, obtained when the derivative is equal to zero, can be expressed as below:

$$
Q^{*}=\sqrt{\frac{D\left[2 s+a D t^{2}\left(\alpha_{c}-\alpha_{d}\right)\right]}{h+a \alpha_{c}}}
$$

- Case 2: The replenishment period never exceeds the trade credit period $(T<t)$. No additional interest charges are to be paid for holding inventory. In this case, the total cost and associated economic order quantity are formulated as follows:

$$
\begin{gathered}
C(\tau)=\frac{s}{\tau}+\frac{D \tau\left(h+a \alpha_{d}\right)}{2}-a D t \alpha_{d} \\
Q^{*}=\sqrt{\frac{2 s D}{h+a \alpha_{d}}}
\end{gathered}
$$


In tactical planning, the aspect of trade credit has been largely investigated in the EOQ model. Jaggi et al. (2008) develop a new inventory model under two levels of trade credit policy with creditlinked demand to reflect real-life situations. Chang et al. (2009) discuss the effects of the inflation rate, the deterioration rate and delay in payment in determining the optimal payment period and the replenishment cycle. Chang et al. (2010) propose an inventory model for deteriorating items with two kind of possible trade credits on the order quantity. Furthermore, Kouvelis \& Zhao (2011) compare short-term debt with supplier-financed trade credit via a newsvendor model and study the interplay between operations and finance. In recent studies, the classical EOQ model has been further developed considering deteriorating items under a two-level trade credit policy. Min et al. (2010) establish a lot-sizing model for deteriorating items with a current-stock-dependent demand and delays in payment in order to maximize a retailer's average profit per unit time. Mo et al. (2014) extend the previous model to a multi-item inventory model for perishable items. The work of Seifert et al. (2013) is referred for the detailed review of trade credit terms that are considered with the EOQ model.

\section{Time value of money and inflation}

The time value of money (TVM) indicates the fact that money values more at the present time than the same amount in the future for its potential earning capacity over time. The TVM is also referred as present discounted value or net present value (NPV). Essentially, money has time value because of the following reasons: first, since risk and uncertainties exist in the future and are extremely difficult to be predicted. A common preference for present consumption generally exists which may result from urgent financial needs and it is to avoid payment in the future. Second, sufficient money at present ensures the availability when facing better investment opportunities. Thus, payment in the future generates an extra opportunity cost. Last, in an inflationary economy, the money received today, has stronger purchasing power than the money to be received in future.

Inflation refers to a continuous increase in the price level and is an index of the prices of all goods in the economic aspect. Actually, inflation occurs when the government creates money at a faster rate than the economy growth. The government should print enough money to fuel economic growth but not too much money that may destroy the value of money. Even though money can potentially generate more money, inflation itself will devalue money that you earn in the future in terms of purchase power. For example, prices for many consumer goods are twice higher than that of 20 years ago.

The TVM and inflation are the first two financial aspects integrated into the EOQ model according to Andriolo et al. (2014). A present value formulation based on the EOQ model is proposed in the early work of Trippi \& Lewin (1974). As presented by Andriolo et al. (2014), this inflation effect is not considered before the study of Buzacott (1975) who considers both the inflation and time value of money in a lot-sizing problem. The author examines the influence of inflation on different types of pricing policies in order to study how TVM impacts inventory decisions. In the work of Trippi \& Lewin (1974), the authors regard the inventory as a form of investment that serves as part of firms' financial structure and is discounted by a continuous-time interest rate, $\alpha_{n p v}$. After substituting the Taylor series expansion, an expression of the optimal order quantity is obtained. The authors then illustrate the insensitivity of the discount cost to the error in the expression. As a result, a near-optimal formulation of the optimal quantity can be written as below:

$$
Q^{*}=\sqrt{\frac{2 D s}{\alpha_{n p v} a}}
$$

In the work of Buzacott (1975), the near-optimal formula considering the inflation is provided using the same up-mentioned reasoning:

$$
Q^{*}=\sqrt{\frac{2 D s}{\left(\alpha_{n p v}-\gamma\right) a}}, \text { where, } \gamma \text { is the inflation rate }
$$


Other formulations can be found in Jesse et al. (1983).

— without inflation

$$
Q^{*}=\sqrt{\frac{2 D s}{\left(h+\alpha_{n p v}\right) a}}
$$

- under inflation

$$
Q^{*}=\sqrt{\frac{2 D s}{\left[h+\left(\alpha_{n p v}-\gamma\right)\right] a}}
$$

During last three decades, this topic is largely investigated in the EOQ literature for more realistic cases. More related works are referred to the review of Andriolo et al. (2014), Taleizadeh \& Nematollahi (2014) and Martínez-Costa et al. (2014). In this work, production planning is executed in a medium-term horizon that the TVM and the inflation effect can thus be neglected.

\section{Cost of capital}

The cost of capital is the rate of return on a portfolio company's existing securities. It is often used to compute the net present value of the cash flows in a proposed investment. Besides, it is also considered to be the minimum after-tax internal rate of return to be earned by new investments. The cost of capital is specific to each particular type of capital that a company uses. At the highest level, they are the cost of equity and the cost of debt, but each class of shares, each class of debt securities, and each loan will have its own cost. The Weighted Average Cost of Capital (WACC) combines all these into a single number for a company's cost of capital.

$W A C C$ : The weighted average cost of capital (WACC) normalizes the cost of capital by combining the interest rates being incurred from both debt and equity. The cost of debt refers to the effective rate that a company pays on its current debt.

$$
\text { Rate }_{\text {debt }}=\frac{I E}{D e}
$$

, where $I E_{t}$ represents interest expenses while $D e$ is the value of total debt.

The cost of equity is the return that stockholders require for their investment in a company.

$$
\text { Rate }_{\text {equity }}=\text { Rate }_{\text {free }}+\beta\left(\text { Rate }_{\text {market }}-\text { Rate }_{\text {free }}\right)
$$

, where Rate $_{\text {free }}$ is the risk-free rate of interest, Rate market $_{\text {is }}$ the expected market return, $\beta$ is to measure the sensitivity of the stock to the market evolution, namely the stock beta, and Rate $e_{\text {market }}-$ Rate $_{\text {free }}$ is the expected risk premium of the stock market. (see in Brealey (2001))

The tax-adjusted formulation can be written as:

$$
W A C C=\text { Rate }_{\text {equity }} \frac{E}{E+D e}+\text { Rate }_{\text {debt }} \frac{D e}{E+D e}\left(1-T_{c}\right)
$$

, where $E$ represents the value of equity, $T_{c}$ is the corporate tax rate. A firm's WACC increases as $\beta$ and the rate of return on equity increase, when an increase in the WACC denotes a decrease in valuation and an increase in risk. A common practice in business admits that the financial part of inventory holding cost is the WACC of the firm (around 12\%) as presented in Serrano et al. (2017).

When defining the cost of capital, it is useful to frame it from either the borrower's point of view (i.e. the organization) or the lender's point of view (the investor). For the organization borrowing the capital, while the cost of capital is the cumulative rate of interest (usually derived as an average rate, combining all capital inputs) applied to the borrowed capital to fund a project. From the investor's point of view, the cost of capital is the relative required return rate considering the risk of the investment being made. 


\subsubsection{Positioning the work of this chapter}

This thesis is relatively close to the literature which involves the cost of capital in operational decision, especially with EOQ-based models. As shown in Zipkin (2000), the cost of capital is added in the Wilson's formula alongside the inventory holding cost. However, it is not frequently considered by the inventory planner as stated by Rumyantsev \& Netessine (2007). Therefore, the authors provide an empirical investigation using the data panel that contains 722 public US companies for the period from 1992 to 2002. Their study shows that classical inventory models continue to provide good policies in an aggregated way at the firm level. Even though the authors prove the influence of the demand uncertainty, company size and margin on the inventory level. No relations can be found between the inventory and the cost of capital since it is affected by the time trend inclusion. This inconclusiveness joins the finding of Lai (2006) which indicates the need of finding new calculation of the cost of capital for operations models will be helpful in the future research. Recent effort of Hoberg et al. (2017) reveals the interplay between a firm's financial situation and its inventory ownership from single- and two-firm perspectives. In their empirical study, the authors propose a refined formulation of the cost of capital and points out that higher costs of capital are weakly associated with lower inventories. Other related studies using the classical way to consider the cost of capital are conducted by Serrano et al. (2009) and Serrano et al. (2017). The former presents the influence of operational decisions on the shareholders' cost of capital. The latter addresses how the cost of capital is more precisely measured when the financial risk depends on the inventory decision made in place of using the WACC.

However, to the best of our knowledge, in the EOQ literature, only a few studies involve the aspect of working capital. Zeballos et al. (2013) explain the evolution of the Modigliani and Miller theorem in the field of inventory management and propose a single-product, finite horizon model to study how different sources of financing and how long-term and short-term debts affect the optimal ordering policy considering the financial aspects of working capital constraints, payment delays and lead times. Although, this model is not exactly based on the EOQ model.

In this work, the financial consequences of the production decision on the production policy are addressed considering the cost of working capital requirement based on the classic EOQ model. The financial cost of the WCR is to cover all costs of production-related operations in medium-term level. It is generated by the time mismatch of paying for the operations and receiving the customer's payment after the payment delay. Since, in the framework of the project RCSM, the planning horizon is defined at the medium-term level, there is thus no NPV and inflation effects being considered in the proposed model.

\subsection{EOQ based profit maximization model, $E O Q_{P(W C R)}$}

\subsubsection{OWCR modeling}

We propose a generic OWCR formulation adapted for the EOQ-based model integrating the financial costs linked to operational decisions (i.e., the financing cost of OWCR). Both the delay in payment to supplier and from client are taken into account in this formulation. To establish the model, we adopt the cash to cash cycle methodology presented in Theodore Farris \& Hutchison (2002) and Hofmann \& Kotzab (2010) where the OWCR for producing a unit of product depends on the amount invested into the related operations (e.g., purchasing, setup, production and inventory holding) and the financing duration of these investments before recovering from corresponding sales. However, we first assume that using the profit portion of the sales revenue to cover the OWCR is not allowed. Since the profit margin of the company has never been required, it does not correspond to the OWCR definition. Furthermore, this assumption supposes that the company does not prioritize its profit allocation to WCR financing. Profit can be allocated to any of a number of objectives for 
the firm including debt reduction, internal or external investment, or dividend payments. Since we do not designate the allocation of profit (to WCR financing or others objectives), our model is thus a partial model for company. Moreover, following the Modigliani Miller Theorem, a company should not affect a specific financial resource (here profit) to a specific object (here WCR financing). In consequence, we adopt the scheme that the WCR generated by producing a product is only effectively recovered when that product is sold. Thus, we progressively and uniformly receive all production related cost from the sales revenue of products over time.

\subsubsection{Assumptions}

In this section, we present an EOQ-based model with a profit maximization objective considering the financing cost of OWCR. The OWCR formulation and the corresponding profit maximization model proposed in this paper are built under the following assumptions :

- Production:

- Demand is constant and uniform during the planning horizon;

- For each production lot, all purchasing, setup, production and inventory holding costs must be financed;

- Production capacity is infinite;

- No backlogging is allowed;

- Only inventory of final product is considered;

- Initial and final stocks are defined as zero;

- No delivery delay of material and production is immediate.

- Financial:

- Production, inventory and setup costs should be paid instantaneously (but may be financed);

- The profit margin from selling products is not be used for financing the WCR in the OWCR calculation;

- The fixed setup cost is earned back uniformly and progressively by selling products of the same production lot.

As previously discussed, we only consider the cost portion in revenue (not the profit margin) for financing the OWCR because the margin may be reinvested in another operation cycle. With this assumption, we do not need to consider the opportunity cost of using the margin to finance the OWCR. Moreover, we consider that the OWCR is generated due to the timing mismatch between revenue and costs to finance. Therefore, we set the last assumption in the list in order to precisely calculate the setup cost in revenue of selling each product. More specifically, all products in the same production lot share the fixed setup cost equally and it will be refunded progressively by selling the products.

\subsubsection{Parameters et decision variables}

Notation is defined for the single-site, single-level, single-product with infinite capacity case in Table 3.1. These parameters are all assumed to be nonnegative. The decision variable is the production lot size, denoted as $Q$. Accordingly, $Q^{*}$ represents the optimal production lot size.

\subsubsection{OWCR formulation}

Following the concept of the cash conversion cycle methodology, we propose a generic OWCR formulation for purchasing, setup, production and holding inventory by taking into account the interest accrued by financing the OWCR during the planning horizon.

In the EOQ model, a uniform time division is established in which, for each cycle, a quantity $Q$ is instantly supplied at the beginning of the cycle and uniformly consumed over the cycle. Thus, for 


\begin{tabular}{ll}
\hline Parameter & Definition \\
\hline$T$ & Horizon length \\
$D$ & Total demand in units \\
$d$ & Demand rate in units per time unit $\left(d=\frac{D}{T}\right)$ \\
$v$ & Unit selling price in dollars per unit \\
$h$ & Unit inventory cost in dollars per unit during a time unit \\
$p$ & Unit production cost in dollars per unit \\
$s$ & Unit setup cost per time in dollars per lot \\
$a$ & Unit raw material cost in dollars per unit \\
$r_{c}$ & Delay in payment from client in time units \\
$r_{f}$ & Delay in payment to supplier in time units \\
$\alpha$ & Interest rate for financing OWCR per dollar per time unit \\
\hline
\end{tabular}

Table 3.1 - Parameters for OWCR modeling

a horizon with total duration, $T$ and a global demand, $D$, each period will last $\frac{Q T}{D}$ time units. Since the OWCR represents the financing need for these operations between when we pay for them and when we collect the payment from customer, these two timings are the key elements of the OWCR measurement. Explicitly, we product a lot of $Q$ products at the beginning of the cycle (i.e., instant 0 ) with the following cost and revenue timing assumptions:

- The setup and production costs are paid immediately, while the purchasing cost is paid at a delay to the supplier at instant $0+r_{f}$.

- A product sold (from inventory) at instant $t \in\left[0, \frac{Q T}{D}\right]$ will be paid at a delay by the customer at the instant $t+r_{c}$.

The three diagrams in Figure 3.3 illustrate the financing needs for purchase, production and setup operations over time. Since the OWCR is the financial need to cover between the timing of paying for expenses and collecting the revenue, the surfaces in these figures represent the OWCR of these operations in each production cycle.
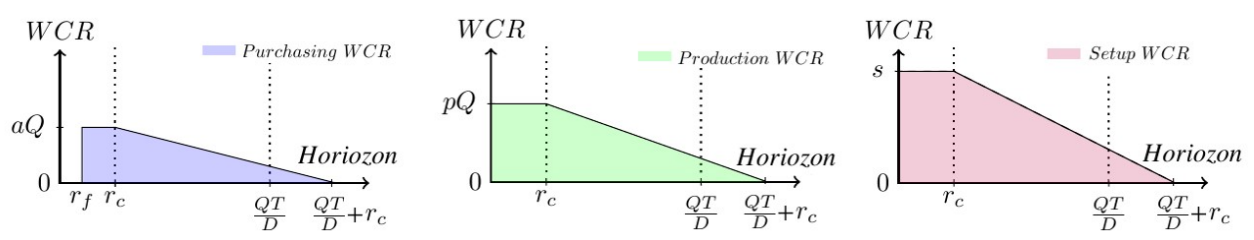

Figure 3.3 - Illustration of OWCR of purchasing, setup and production costs in a cycle

We can then easily establish the formulation of the OWCR for each lot, generated by purchasing, setup and production, as follows:

$$
\begin{aligned}
W C R_{\text {purchasing }}(Q) & =a\left[Q\left(r_{c}-r_{f}\right)+\frac{Q^{2} T}{2 D}\right] \\
W C R_{\text {setup }}(Q) & =s\left(r_{c}+\frac{Q T}{2 D}\right) \\
W C R_{\text {production }}(Q) & =p\left(Q r_{c}+\frac{Q^{2} T}{2 D}\right)
\end{aligned}
$$

Contrary to purchasing, set-up and production, which are all one-time payments, a unitary inventory holding cost must be paid regularly for each item for the entire duration of its storage. 
Therefore, there is a cumulative effect in financing the inventory holding costs. More precisely, for one product sold at $t \in\left[0, \frac{Q T}{D}\right]$, we must pay its inventory holding costs generated in all instants over the entire duration from 0 to $t$. Thus, all these costs must be financed from the instant when they occur (instant 0) until the arrival of client's payment at $t+r_{c}$, as presented in Figure 3.4 .

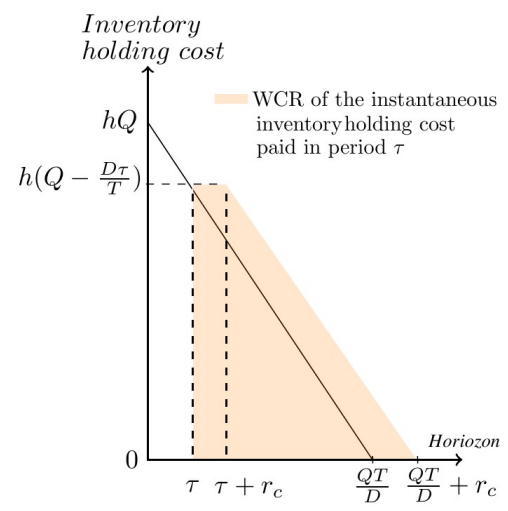

Figure 3.4 - Illustration of the OWCR calculation for inventory holding cost

Consider an item that is sold at insant $t$, where $\left.t \in\left[0, \frac{Q T}{D}\right]\right)$ and one of its inventory payment which occurs at $\tau \in[0, t]$. This cost must be financed until the customer pays the product at $t+r_{c}$. Therefore, the financing duration will be $t+r_{c}-\tau$. Consequently, the OWCR for inventory holding cost in a production cycle is formulated as follows:

$$
\begin{aligned}
W C R_{\text {inventory }}(Q) & =\int_{0}^{\frac{Q T}{D}} \int_{0}^{t} h \frac{Q T}{D}\left(t+r_{c}-\tau\right) d \tau d t \\
& =h\left(\frac{Q^{3} T^{2}}{6 D^{2}}+\frac{r_{c} Q^{2} T}{2 D}\right)
\end{aligned}
$$

Following the above formulations of OWCR, the total OWCR for a production cycle can be expressed as follows:

$$
\begin{aligned}
W C R(Q) & =W C R_{\text {purchasing }}+W C R_{\text {setup }}+W C R_{\text {production }}+W C R_{\text {inventory }} \\
& =h\left(\frac{Q^{3} T^{2}}{6 D^{2}}+\frac{r_{c} Q^{2} T}{2 D}\right)+a\left[Q\left(r_{c}-r_{f}\right)+\frac{Q^{2} T}{2 D}\right]+p\left(r_{c} Q+\frac{Q^{2} T}{2 D}\right)+s\left(r_{c}+\frac{Q T}{2 D}\right) \\
& =\frac{h T^{2}}{6 D^{2}} Q^{3}+\frac{T}{2 D}\left(h r_{c}+a+p\right) Q^{2}+\left[a\left(r_{c}-r_{f}\right)+p r_{c}+\frac{s T}{2 D}\right] Q+s r_{c}
\end{aligned}
$$

Accordingly, the total WCR is formulated as follows:

$$
\begin{aligned}
W C R_{\text {total }}= & \frac{D}{Q} \times W C R(Q)=\frac{h T^{2}}{6 D} Q^{2}+\frac{T}{2}\left(h r_{c}+a+p\right) Q+D\left[a\left(r_{c}-r_{f}\right)+p r_{c}+\frac{s T}{2 D}\right] \\
& +s r_{c} D \frac{1}{Q}
\end{aligned}
$$

\subsubsection{Objective function}

The aim of our contribution is to maximize the profit by satisfying constant demands. According to the profit definition proposed by Chen \& Teng (2015), the profit can be defined as the difference between sales revenue and all expenses including logistic costs and interests to pay. In our case, the interest is the cost of financing the OWCR. The formulations of these components are

- The sales revenue (denoted as $R$ ): $R=v D$; 
- The logistic cost (denoted as $C L$ ) is the sum of purchasing, setup, production and inventory holding costs: $C L=a D+s \frac{D}{Q}+p D+h T \frac{Q}{2}$;

- The interest to pay for financing the OWCR (denoted as $C F$ ) based on an interest rate $\alpha$ : $C F=\alpha W C R_{\text {total }}$.

Consequently, the objective function is formulated as

$$
\begin{aligned}
\text { Profit }= & R-C L-C F \\
= & v D-\left(a D+s \frac{D}{Q}+p D+h T \frac{Q}{2}\right) \\
& -\alpha\left\{\frac{h Q^{2} T^{2}}{6 D}+\frac{Q T}{2}\left(h r_{c}+a+p\right)+D\left[a\left(r_{c}-r_{f}\right)+p r_{c}+\frac{s T}{2 D}\right]+\frac{s r_{c} D}{Q}\right\} \\
= & D\left\{v-a-p-\alpha\left[a\left(r_{c}-r_{f}\right)+p r_{c}+\frac{s T}{2 D}\right]\right\} \\
& -\frac{h T+\alpha\left(h r_{c}+a+p\right) T}{2} Q-\frac{\alpha h T^{2}}{6 D} Q^{2}-\frac{D s\left(1+\alpha r_{c}\right)}{Q}
\end{aligned}
$$

We observe that the objective function is concave because all negative terms are either constant, linearly increase or concave which means a unique maximum solution exists. In the context of this work, since we assume a constant sales unit price, the revenue is rather constant and independent of the production quantity. This profit maximization objective is thus equivalent to the cost minimization objective (i.e. minimization of $C L+C F$ ). However, this conversion may not remain valid in a more general case, for example, with consideration of the time value of money.

\subsection{Optimal solution and structural properties}

To calculate the optimal production lot size, $Q^{*}$, we take the first-order derivative of Profit.

$$
\begin{aligned}
\frac{\mathbf{d} \text { Profit }}{\mathbf{d} Q} & =-\frac{s D}{Q^{2}}+\frac{h T}{2}+\frac{2 \alpha h Q T^{2}}{6 D}+\frac{\alpha\left(h r_{c}+a+p\right) T}{2}-\frac{\alpha D s r_{c}}{Q^{2}} \\
& =-\frac{s\left(1+\alpha r_{c}\right) D}{Q^{2}}+\frac{\alpha h Q T^{2}}{3 D}+\frac{\left[h+\alpha\left(h r_{c}+a+p\right)\right] T}{2}
\end{aligned}
$$

\subsubsection{Optimal solution}

The optimal solution is obtained when the equation (3.17) is set equal to zero. In order to calculate it, we adopt the Cardano method which is devoted to resolve a cubic equation in a special form.

Proposition 3.4.1. We denote that $\Delta=\frac{3 \alpha^{2} h^{2} T^{4}}{s^{2}\left(1+\alpha r_{c}\right)^{2} D^{4}}-\frac{\left[h+\alpha\left(h r_{c}+a+p\right)\right]^{3} T^{3}}{2 s^{3}\left(1+\alpha r_{c}\right)^{3} D^{3}}$

$$
\begin{aligned}
& \text { - if } \Delta>0 \text {, }
\end{aligned}
$$

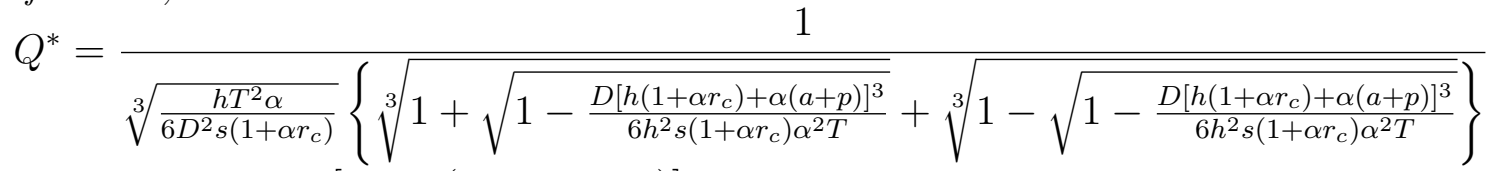

$$
\begin{aligned}
& \text { - if } \Delta=0, Q^{*}=\frac{D\left[h+\alpha\left(h r_{c}+a+p\right)\right]}{2 \alpha h T}
\end{aligned}
$$


- if $\Delta<0$,

$$
Q^{*}=\frac{1}{2 \sqrt{\frac{\left[h\left(1+\alpha r_{c}\right)+\alpha(a+p)\right] T}{6 D s\left(1+\alpha r_{c}\right)}} \cos \left\{\frac{1}{3} \arccos \left[\sqrt{\left.\frac{6 h^{2} s\left(1+\alpha r_{c}\right) \alpha^{2} T}{\left[h\left(1+\alpha r_{c}\right)+\alpha(a+p)\right]^{3} D}\right]}\right\}\right.}
$$

Proof. We seek to maximize the objective function given by:

$$
\begin{aligned}
\text { Profit }= & D\left\{v-a-p-\alpha\left[a\left(r_{c}-r_{f}\right)+p r_{c}+\frac{s T}{2 D}\right]\right\}-\frac{h T+\alpha\left(h r_{c}+a+p\right) T}{2} Q \\
& -\frac{\alpha h T^{2}}{6 D} Q^{2}-\frac{D s\left(1+\alpha r_{c}\right)}{Q}
\end{aligned}
$$

The objective function is strictly concave as a sum of concave functions and a strictly concave function $(Q>0)$. Thus, if there is a maximum, it will be unique and will occur when function (3.17) will be equal to zero. Thus,

$$
\begin{aligned}
\frac{\mathbf{d} \text { Profit }}{\mathbf{d} Q}=0 & \Leftrightarrow-\frac{s\left(1+\alpha r_{c}\right) D}{Q^{2}}+\frac{\alpha h Q T^{2}}{3 D}+\frac{\left[h+\alpha\left(h r_{c}+a+p\right)\right] T}{2}=0 \\
\left(Q \in \mathbb{R}_{+}^{*}\right) & \Leftrightarrow-\frac{s\left(1+\alpha r_{c}\right) D}{Q^{3}}+\frac{\left[h+\alpha\left(h r_{c}+a+p\right)\right] T}{2 Q}+\frac{\alpha h T^{2}}{3 D}=0 \\
\left(X=\frac{1}{Q}\right) & \Leftrightarrow s\left(1+\alpha r_{c}\right) D X^{3}-\frac{\left[h+\alpha\left(h r_{c}+a+p\right)\right] T}{2} X-\frac{\alpha h T^{2}}{3 D}=0 \\
\left(s\left(1+\alpha r_{c}\right) D>0\right) & \Leftrightarrow X^{3}-\frac{\left[h+\alpha\left(h r_{c}+a+p\right)\right] T}{2 s\left(1+\alpha r_{c}\right) D} X-\frac{\alpha h T^{2}}{3 s\left(1+\alpha r_{c}\right) D^{2}}=0
\end{aligned}
$$

We compute the discriminant of this cubic equation. According to Cardano's method, for a cubic equation, $x^{3}+p x+q=0$, the discriminant $\Delta=4 p^{3}+27 q^{2}$. Therefore, for our case, $p=$ $-\frac{\left[h+\alpha\left(h r_{c}+a+p\right)\right] T}{2 s\left(1+\alpha r_{c}\right) D}$ and $q=-\frac{\alpha h T^{2}}{3 s\left(1+\alpha r_{c}\right) D^{2}}$. Consequently,

$$
\Delta=-4\left\{\frac{\left[h+\alpha\left(h r_{c}+a+p\right)\right] T}{2 s\left(1+\alpha r_{c}\right) D}\right\}^{3}+27\left[-\frac{\alpha h T^{2}}{3 s\left(1+\alpha r_{c}\right) D^{2}}\right]^{2}
$$

Three cases must be considered:

$-\Delta>0$ : In this case, there is only one real root given by:

$$
X^{*}=\frac{1}{3}\left\{\sqrt[3]{\frac{\frac{27 \alpha h T^{2}}{3 s\left(1+\alpha r_{c}\right) D^{2}}+27 \sqrt{\Delta}}{2}}+\sqrt[3]{\frac{\frac{27 \alpha h T^{2}}{3 s\left(1+\alpha r_{c}\right) D^{2}}-27 \sqrt{\Delta}}{2}}\right\}
$$

Thus,

$$
\begin{aligned}
& Q^{*}=\frac{1}{X^{*}}=\frac{1}{\sqrt[3]{\frac{h T^{2} \alpha}{6 D^{2} s\left(1+\alpha r_{c}\right)}}} \times \\
& {\left[\sqrt[3]{1+\sqrt{1-\frac{D\left[h\left(1+\alpha r_{c}\right)+\alpha(a+p)\right]^{3}}{6 h^{2} s\left(1+\alpha r_{c}\right) \alpha^{2} T}}}+\sqrt[3]{1-\sqrt{1-\frac{D\left[h\left(1+\alpha r_{c}\right)+\alpha(a+p)\right]^{3}}{6 h^{2} s\left(1+\alpha r_{c}\right) \alpha^{2} T}}}\right]}
\end{aligned}
$$


$-\Delta=0:$ There are two real roots:

$$
\begin{aligned}
& X_{1}^{*}=\frac{3 \alpha h T^{2}}{3 s\left(1+\alpha r_{c}\right) D^{2}} \times \frac{2 s\left(1+\alpha r_{c}\right) D}{\left[h+\alpha\left(h r_{c}+a+p\right)\right] T}=\frac{2 \alpha h T}{D\left[h+\alpha\left(h r_{c}+a+p\right)\right]}>0 \\
& X_{2}^{*}=-\frac{3 \alpha h T^{2}}{3 s\left(1+\alpha r_{c}\right) D^{2}} \times \frac{s\left(1+\alpha r_{c}\right) D}{\left[h+\alpha\left(h r_{c}+a+p\right)\right] T}=\frac{-2 \alpha h T}{D\left[h+\alpha\left(h r_{c}+a+p\right)\right]}<0
\end{aligned}
$$

Thus,

$$
Q^{*}=\frac{1}{X_{1}^{*}}=\frac{D\left[h(1+\alpha)+\alpha\left(h r_{c}+a+p\right)\right]}{2 \alpha h T}
$$

$-\Delta<0$ : In this case, there are three real roots. Nevertheless, due to the strict concavity of the objective function in $\mathbb{R}_{+}^{*}$, there will be only one positive root. The three real roots are given by:

$$
\begin{aligned}
X_{k}^{*}=2 \sqrt{\frac{\left[h\left(1+\alpha r_{c}\right)+\alpha(a+p)\right] T}{6 D s\left(1+\alpha r_{c}\right)}} \times \\
\quad \cos \left[\frac{1}{3} \arccos \left(\sqrt{\frac{6 h^{2} s\left(1+\alpha r_{c}\right) \alpha^{2} T}{\left[h\left(1+\alpha r_{c}\right)+\alpha(a+p)\right]^{3} D}}+\frac{2 k \pi}{3}\right)\right], \text { where } k \in\{0,1,2\}
\end{aligned}
$$

As $\Delta<0$, then we can deduce that

$$
\begin{aligned}
& 4\left\{\frac{\left[h+\alpha\left(h r_{c}+a+p\right)\right] T}{2 s\left(1+\alpha r_{c}\right) D}\right\}^{3}>27\left[-\frac{\alpha h T^{2}}{3 s\left(1+\alpha r_{c}\right) D^{2}}\right]^{2} \\
\Leftrightarrow & \frac{6 h^{2} s\left(1+\alpha r_{c}\right) \alpha^{2}}{\left[h\left(1+\alpha r_{c}\right)+\alpha(a+p)\right]^{3}} \frac{T}{D}<1 \\
\Rightarrow & \sqrt{\frac{6 h^{2} s\left(1+\alpha r_{c}\right) \alpha^{2}}{\left[h\left(1+\alpha r_{c}\right)+\alpha(a+p)\right]^{3}} \frac{T}{D}}<1
\end{aligned}
$$

As a consequence, $0<\frac{1}{3} \arccos \left(\sqrt{\frac{6 h^{2} s\left(1+\alpha r_{c}\right) \alpha^{2} T}{\left[h\left(1+\alpha r_{c}\right)+\alpha(a+p)\right]^{3} D}}\right)<\frac{\pi}{6}$. Therefore, we obtain negative values of $\cos \left[\frac{1}{3} \arccos \left(\sqrt{\frac{6 h^{2} s\left(1+\alpha r_{c}\right) \alpha^{2} T}{\left[h\left(1+\alpha r_{c}\right)+\alpha(a+p)\right]^{3} D}}+\frac{2 k \pi}{3}\right)\right]$ when $k=1$ or 2 . Thus, the only positive root is $X_{0}^{*}$ in this case. Then,

$$
Q^{*}=\frac{1}{X_{0}^{*}}=\frac{1}{2 \sqrt{\frac{\left[h\left(1+\alpha r_{c}\right)+\alpha(a+p)\right] T}{6 D s\left(1+\alpha r_{c}\right)}} \cos \left\{\frac{1}{3} \arccos \left[\sqrt{\frac{6 h^{2} s\left(1+\alpha r_{c}\right) \alpha^{2} T}{\left[h\left(1+\alpha r_{c}\right)+\alpha(a+p)\right]^{3} D}}\right]\right\}}
$$

We can immediately remark that the optimal solution does not depend on the delay in payment to the supplier. This is not surprising as it remains constant whatever the value of $\mathrm{Q}$ in the OWCR formulation. We can also deduce from this proposition the two following corollaries.

Corollary 3.4.1.1. If $\alpha=0, Q^{*}=\sqrt{\frac{2 D s}{h T}}$. Thus, by considering the interest to pay for financing the OWCR, our result extends the Wilson formula. 
Proof. It suffices to remark that if $\alpha=0$, then $\Delta<0$ and $\cos \frac{\pi}{6}=\frac{\sqrt{3}}{2}$.

Corollary 3.4.1.2. For the optimal production quantity, there is an equilibrium between (1) the setup costs and their associated financing costs during the delay of payment from client and (2) the holding costs and their associated financing costs during the delay of payment from client plus the financing cost of purchasing and productions cost during the planning horizon.

Proof. The optimality occurs when $(3.17)=0$. Consequently,

$$
\frac{D}{Q} s\left(1+\alpha r_{c}\right)=\frac{Q}{2} T\left[h+\alpha\left(h r_{c}+a+p\right)\right]+\alpha \frac{h T^{2}}{3 D} Q^{2}
$$

Compared to the trade-off in the classic EOQ problem, considering the financial cost leads to a cumulative effect on $W C R_{\text {inventory }}$. Therefore, our proposed model favors the inventory holding cost much more in the classic trade-off versus the setup cost. Moreover, we must also consider the minor influence given by the financial cost of purchasing and production costs due to the small value of the interest rate.

Proposition 3.4.2. For any value of $\Delta$,

$-Q^{*}$ is an increasing function of $s$ and $r_{c}$

- $Q^{*}$ is a decreasing function $h, a, p$ and $\alpha$

Proof. In order to analyze the variation of the optimal order quantity with varying parameters, we focus on the first derivative of the objective function at optimality ( by

$$
-\frac{s\left(1+\alpha r_{c}\right) D}{Q^{* 2}}+\frac{\alpha h Q^{*} T^{2}}{3 D}+\frac{\left[h+\alpha\left(h r_{c}+a+p\right)\right] T}{2}=0
$$

In the following analysis, only one of the parameters is varied at a time and we will be able to deduce the variation of $Q^{*}$ for keeping the equation valid. To do so, the equation (3.19) can be rewritten as follows:

$$
D s=\left[\frac{T}{3\left(\frac{1}{\alpha}+r_{c}\right) D} Q^{* 3}+\frac{1}{2} Q^{* 2}\right] T h+\frac{(a+p) T}{2\left(\frac{1}{\alpha}+r_{c}\right)} Q^{* 2}
$$

By examining this equation, we observe that

- If $s$ increases, then $Q^{*}$ will increase to maintain the equality because $D$ and the coefficients of all terms of right side of the equation (3.20) are fixed;

- If $h$ increases, then $Q^{*}$ have to decrease because $D s, \frac{T}{3\left(\frac{1}{\alpha}+r_{c}\right) D}$ and $\frac{(a+p) T}{2\left(\frac{1}{\alpha}+r_{c}\right)}$ are constant;

- If $a$ or $p$ increases, then $Q^{*}$ must decrease because $D s$ and the coefficient of the other terms remain the same;

- If $r_{c}$ increases, then $Q^{*}$ must increase because $\frac{T}{3\left(\frac{1}{\alpha}+r_{c}\right) D}$ and $\frac{(a+p) T}{2\left(\frac{1}{\alpha}+r_{c}\right)}$ will decrease plus $D s$ and $T h$ are the same;

- if $\alpha$ increases, then $Q^{*}$ will inversely decrease proportionally as in the previous case.

The Table 3.2 summarizes these results.

Proposition 3.4.3. For any value of $\Delta$, let $Q_{E O Q}^{*}$ be the optimal solution for the classic EOQ model, then $Q^{*} \leq Q_{E O Q}^{*}=\sqrt{\frac{2 D s}{h T}}$ 


\begin{tabular}{c|c|c}
\hline Parameter & Variation & Influence on $\mathrm{Q}^{*}$ \\
\hline$s$ & $\nearrow$ & $\nearrow$ \\
\hline$r_{c}$ & $\nearrow$ & $\nearrow$ \\
\hline$h$ & $\nearrow$ & $\searrow$ \\
\hline$a$ & $\nearrow$ & $\searrow$ \\
\hline$p$ & $\nearrow$ & $\searrow$ \\
\hline$\alpha$ & $\nearrow$ & $\searrow$ \\
\hline
\end{tabular}

Table 3.2 - Analysis of influences of varying parameters on optimal batch size

Proof. In order to compare between the optimal solutions of this model and of the classic EOQ model (Denoted as $Q_{E O Q}^{*}$ ), the equation $(3.20)$ is reformulated as follows.

$$
\begin{aligned}
D s & =\left[\frac{T}{3\left(\frac{1}{\alpha}+r_{c}\right) D} Q^{* 3}+\frac{1}{2} Q^{* 2}\right] T h+\frac{(a+p) T}{2\left(\frac{1}{\alpha}+r_{c}\right)} Q^{* 2} \\
\Leftrightarrow D s & =\left[\frac{h T}{2}+\frac{h T^{2} Q^{*}}{3\left(\frac{1}{\alpha}+r_{c}\right) D}+\frac{(a+p) T}{2\left(\frac{1}{\alpha}+r_{c}\right)}\right] Q^{* 2} \\
\Leftrightarrow Q^{*} & =\sqrt{\frac{2 D s}{h T+\frac{2 h T^{2} Q^{*}}{3\left(\frac{1}{\alpha}+r_{c}\right) D}+\frac{(a+p) T}{\left(\frac{1}{\alpha}+r_{c}\right)}}}
\end{aligned}
$$

Since $\frac{2 h T^{2} Q^{*}}{3\left(\frac{1}{\alpha}+r_{c}\right) D}+\frac{(a+p) T}{\left(\frac{1}{\alpha}+r_{c}\right)}$ can only be a positive value, we are able to obtain that $Q^{*} \leq$ $\sqrt{\frac{2 D s}{h T}}=Q_{E O Q}^{*}$. This proposition shows that taking into account the interest generated by financing the OWCR during the planning horizon always leads to a decrease of the optimum batch size compared with the classical Economic Order Quantity.

Corollary 3.4.3.1. The optimal production quantity obtained by the proposed model is less sensitive to variation of the fixed setup cost and of the inventory holding unit cost compared with the optimal quantity of classic EOQ model

Proof. In Corollary 3.4.3.1, we study the sensibility of the optimal production quantities with varying parameter of fixed setup cost and inventory holding unit cost. In order to compare to EOQ model, we set the values of purchasing and production unit cost at zero. Then, we focus on the trade-offs in this problem and the $E O Q$ problem which are respectively presented as follows:

- In the $E O Q_{P(W C R)}$ problem, the trade-off is obtained when the equation is equal to zero. Thus,

$$
\begin{gathered}
\frac{s\left(1+\alpha r_{c}\right) D}{Q^{* 2}}=\frac{\alpha h T^{2} Q^{*}}{3 D}+\frac{h T\left(1+\alpha r_{c}\right)}{2} \\
s D=\frac{h T Q^{* 2}}{2}+\frac{\alpha h T^{2} Q^{* 3}}{3 D\left(1+\alpha r_{c}\right)}
\end{gathered}
$$

- In EOQ problem, the trade-off can be formulated as follows:

$$
s D=\frac{h T Q^{* 2}}{2}
$$


Let denote that $Q^{*}$ and $Q_{E O Q}^{*}$ are the optimal quantities obtained by $E O Q_{P(W C R)}$ model and $E O Q$ model. With zero purchasing and production unit cost, the equation (3.21) is expressed as follow:

$$
\begin{aligned}
Q^{*} & =\sqrt{\frac{2 D s}{h T+\frac{2 h T^{2} Q^{*}}{3\left(\frac{1}{\alpha}+r_{c}\right) D}}} \\
\Leftrightarrow Q^{*} & =\sqrt{\frac{2 D s}{h T\left[1+\frac{2 \alpha T Q^{*}}{3 D\left(1+\alpha r_{c}\right)}\right]}}
\end{aligned}
$$

Since $Q_{E O Q}^{*}=\sqrt{\frac{2 D s}{h T}}$, then

$$
\begin{aligned}
\frac{Q_{E O Q}^{* 2}}{Q^{* 2}} & =\left\{\frac{\sqrt{\frac{2 D s}{h T}}}{\sqrt{\frac{2 D s}{h T\left[1+\frac{2 \alpha T Q^{*}}{3 D\left(1+\alpha r_{c}\right)}\right]}}}\right\}^{2} \\
& =1+\frac{2 \alpha T Q^{*}}{3 D\left(1+\alpha r_{c}\right)}>1
\end{aligned}
$$

This equation shows the relation between optimal quantity of these models with same set of instance. Next, with the same value of parameters expect for $s$, we denote that $Q_{1}^{*}\left(Q_{2}^{*}\right)$ and $Q_{E O Q 1}^{*}$ $\left(Q_{E O Q 2}^{*}\right)$ are the optimal quantities with $s_{1}\left(s_{2}\right.$, where $\left.s_{2}>s_{1}\right)$. According to the proposition 3.4 .2 , the optimal quantities in our problem will increase following the increase of $s$, i.e. $Q_{1}^{*}<Q_{2}^{*}$. Same relation can be found in EOQ problem, i.e. $Q_{E O Q 1}^{*}<Q_{E O Q 2}^{*}$. We express the relation between these optimal quantity in these two cases:

$$
\begin{aligned}
\frac{Q_{E O Q 1}^{* 2}}{Q_{1}^{* 2}} & =1+\frac{2 \alpha T Q_{1}^{*}}{3 D\left(1+\alpha r_{c}\right)} \\
\frac{Q_{E O Q 2}^{* 2}}{Q_{2}^{* 2}} & =1+\frac{2 \alpha T Q_{2}^{*}}{3 D\left(1+\alpha r_{c}\right)}
\end{aligned}
$$

Since $Q_{1}^{*}<Q_{2}^{*}$, we have $\frac{2 \alpha T Q_{1}^{*}}{3 D\left(1+\alpha r_{c}\right)}<\frac{2 \alpha T Q_{2}^{*}}{3 D\left(1+\alpha r_{c}\right)}$. We can thus deduce that $\frac{Q_{E O Q 1}^{*}}{Q_{1}^{*}}<\frac{Q_{E O Q 2}^{*}}{Q_{2}^{*}}$. Then, we assume that $X=Q_{E O Q 1}^{*} \frac{Q_{2}^{*}}{Q_{1}^{*}}$. According to the proposition 3.4.3, $Q_{1}^{*}<Q_{E O Q 1}^{*}$ and we get:

$$
\frac{X-Q_{E O Q 1}^{*}}{Q_{2}^{*}-Q_{1}^{*}}=\frac{Q_{E O Q 1}^{*}}{Q_{1}^{*}} \frac{\frac{Q_{2}^{*}}{Q_{1}^{*}}-1}{\frac{Q_{2}^{*}}{Q_{1}^{*}}-1}>1
$$

Thus, $X-Q_{E O Q 1}^{*}>Q_{2}^{*}-Q_{1}^{*}$. Furthermore, we study the relation between $Q_{E O Q 2}^{*}$ and $X$. Because 
we have $\frac{Q_{E O Q 1}^{*}}{Q_{1}^{*}}<\frac{Q_{E O Q 2}^{*}}{Q_{2}^{*}}$ which means $Q_{E O Q 2}^{*} Q_{1}^{*}>Q_{E O Q 1}^{*} Q_{2}^{*}$, we obtain that

$$
\begin{aligned}
Q_{E O Q 2}^{*}-X & =Q_{E O Q 2}^{*}-Q_{E O Q 1}^{*} \frac{Q_{2}^{*}}{Q_{1}^{*}} \\
& =\frac{Q_{E O Q 2}^{*} Q_{1}^{*}-Q_{E O Q 1}^{*} Q_{2}^{*}}{Q_{1}^{*}}>0
\end{aligned}
$$

To conclude, $Q_{E O Q 2}^{*}-Q_{E O Q 1}^{*}>Q_{2}^{*}-Q_{1}^{*}$. It signifies that with the same increase of the fixed setup cost, the optimal quantity in EOQ model will increase more than in our model which represents that it is more sensible to the $s$ in our model than in EOQ model.

Similar argument can be found for the inventory holding unit cost, $h$. The difference is, according to the proposition 3.4.2, when $h$ increases, the optimal quantity will decrease in our model (same in EOQ model). In this case, we fix all parameters expect for $h$ (in place of $s$ in the previous case). Therefore, the relations between up-mentioned optimal quantities are $Q_{E O Q 1}^{*}>Q_{E O Q 2}^{*}$ and $Q_{1}^{*}>Q_{2}^{*}$. Comparing between the equation $\sqrt{3.25}$ and $\sqrt{3.26}$, we obtain $\frac{Q_{E O Q 1}^{*}}{Q_{1}^{*}}>\frac{Q_{E O Q 2}^{*}}{Q_{2}^{*}}$ because $Q_{1}^{*}>Q_{2}^{*}$. We execute similar approach as in the previous case of $s$. We assume that $X=Q_{E O Q 1}^{*} \frac{Q_{2}^{*}}{Q_{1}^{*}}$. According to proposition 3.4 .3 , we have $Q_{E O Q 1}^{*}>Q_{E O Q 2}^{*}$. Then, we get:

$$
\frac{Q_{E O Q 1}^{*}-X}{Q_{1}^{*}-Q_{2}^{*}}=\frac{Q_{E O Q 1}^{*}}{Q_{1}^{*}} \frac{\frac{1-Q_{2}^{*}}{Q_{1}^{*}}}{\frac{1-Q_{2}^{*}}{Q_{1}^{*}}}>1
$$

Thus, $Q_{E O Q 1}^{*}-X>Q_{1}^{*}-Q_{2}^{*}$. Subsequently, we compare the relation between $X$ and $Q_{E O Q 2}^{*}$ with the relation $\frac{Q_{E O Q 1}^{*}}{Q_{1}^{*}}>\frac{Q_{E O Q 2}^{*}}{Q_{2}^{*}}$.

$$
\begin{aligned}
X-Q_{E O Q 2}^{*} & =Q_{E O Q 1}^{*} \frac{Q_{2}^{*}}{Q_{1}^{*}}-Q_{E O Q 2}^{*} \\
& =\frac{Q_{E O Q 1}^{*} Q_{2}^{*}-Q_{E O Q 2}^{*} Q_{1}^{*}}{Q_{1}^{*}}>0
\end{aligned}
$$

Therefore, $Q_{E O Q 1}^{*}-Q_{E O Q 2}^{*}>Q_{1}^{*}-Q_{2}^{*}$. We thus obtain similar result that the optimal quantity, $Q^{*}$, is less sensible to the $h$ than $Q_{E O Q}^{*}$.

\subsubsection{Comparison with classic EOQ based-formula with the cost of cap- ital}

In the literature and in practice, a traditional way to consider the cost of capital is to add it alongside the holding cost in Wilson's formula. For instance, as Jesse et al. (1983) proposes, in an inventory management context, the near-optimal formula considering the cost of capital can be written as $Q_{j}^{*}=\sqrt{\frac{2 D s}{h T+\alpha_{c} V T}}$ where $V$ represents unitary product value (unit cost in their work) and $\alpha_{c}$ as the cost of capital. As is customary in the EOQ literature, the unit cost is the sum of 
operation costs including purchasing raw material and production which is $V=a+p$. The setup is not included in the unit cost (Harris (1913)). Therefore, the approximate formula can be rewritten as :

$$
Q_{j}^{*}=\sqrt{\frac{2 D s}{\left[h+\alpha_{c}(a+p)\right] T}}
$$

Note that if we take equation (4.37) and set $r_{c}=0$ and remove the last term containing the quadratic variables we will obtain the approximation in exact same form as given in (3.31) (i.e. $\alpha$ in place of $\alpha_{c}$ ). We then seek to compare this approximation with our fully derived formula taking into account WCR financing from Proposition 3.4.1 without client payment delay as given in the following proposition.

Proposition 3.4.4. When $r_{c}=0$, the ratio between optimal production quantities obtained with our formula (proposition 3.4.1), denoted as $Q^{*}$, and with classic EOQ-based formula considering cost of capital, denoted as $Q_{j}^{*}$, can be expressed in different cases as follows :

- if $\Delta>0,0<\frac{Q^{*}}{Q_{j}^{*}}<\frac{\sqrt{3}}{2}$.

- if $\Delta=0, \frac{Q^{*}}{Q_{j}^{*}}=\frac{\sqrt{3}}{2}$

- if $\Delta<0, \frac{\sqrt{3}}{2}<\frac{Q^{*}}{Q_{j}^{*}}<1$.

Proof.

We compare the ratio between the optimal production quantities obtained by two formula with $r_{c}=0$ in three cases. These cases value of $\Delta$. Knowing that $\Delta=-4\left\{\frac{[h+\alpha(a+p)] T}{2 s D}\right\}^{3}+$ $27\left[-\frac{\alpha h T^{2}}{3 s D^{2}}\right]^{2}$, the three cases are discussed in the following paragraphs.

- When $\Delta>0$, the ratio can be expressed as follows :

$$
\begin{aligned}
\frac{Q^{*}}{Q_{j}^{*}}=\sqrt{3} \sqrt[6]{\frac{D[h+\alpha(a+p)]^{3}}{6 \alpha^{2} h^{2} s T}} & \times \\
& \frac{1}{\sqrt[3]{1+\sqrt{1-\frac{D[h+\alpha(a+p)]^{3}}{6 \alpha^{2} h^{2} s T}}}+\sqrt[3]{1-\sqrt{1-\frac{D[h+\alpha(a+p)]^{3}}{6 \alpha^{2} h^{2} s T}}}}
\end{aligned}
$$

Denote that $X=\frac{D[h+\alpha(a+p)]^{3}}{6 \alpha^{2} h^{2} s T}$ where $0<X<1$ since $\Delta>0$. Consequently, $\frac{Q^{*}}{Q_{j}^{*}}=$ $\sqrt{3} F(X)=\frac{\sqrt{3} \sqrt[6]{X}}{\sqrt[3]{1+\sqrt{1-X}}+\sqrt[3]{1-\sqrt{1-X}}}$. Then, we search the extreme value of this equation by calculating the derivate of $F(X)$. 


$$
\begin{aligned}
\frac{[F(X)]}{X}= & \frac{1}{6(\sqrt[3]{\sqrt{1-X}+1}+\sqrt[3]{1-\sqrt{1-X}}) x^{\frac{5}{6}}} \\
& -\frac{\left(\frac{1}{6(1-\sqrt{1-X})^{\frac{2}{3}} \sqrt{1-X}}-\frac{1}{6(\sqrt{1-X}+1)^{\frac{2}{3}} \sqrt{1-X}}\right) \sqrt[6]{X}}{(\sqrt[3]{\sqrt{1-X}+1}+\sqrt[3]{1-\sqrt{1-X}})^{2}} \\
= & \frac{\sqrt{1-X}-\sqrt[3]{X}(\sqrt[3]{\sqrt{1-X}+1}-\sqrt[3]{1-\sqrt{1-X}})}{6(\sqrt[3]{\sqrt{1-X}+1}+\sqrt[3]{1-\sqrt{1-X}}) X^{\frac{5}{6}} \sqrt{1-X}}
\end{aligned}
$$

Since we have $0<X<1$, we attempt to determinate the sign of the derivate. First, the denominator of $(3.32)$ is greater than zero with $0<X<1$. Second, for the nominator, we let $Y=\sqrt{1-X}$ where $0<Y<1$. Therefore, the nominator of (.11) can be rewritten as $N(Y)=Y-\sqrt[3]{1-Y^{2}}(\sqrt[3]{1+Y}-\sqrt[3]{1-Y})$. Its derivate is calculated as follows:

$$
\frac{N(Y)}{Y}=\frac{2 Y(\sqrt[3]{Y+1}-\sqrt[3]{1-Y})}{3\left(1-Y^{2}\right)^{\frac{2}{3}}}-\sqrt[3]{1-Y^{2}}\left(\frac{1}{3(Y+1)^{\frac{2}{3}}}+\frac{1}{3(1-Y)^{\frac{2}{3}}}\right)+1
$$

In order to determinate the sign of $\frac{N(Y)}{Y}$, we denote that $A=\sqrt[3]{1+Y}$ and $B=\sqrt[3]{1-Y}$. With this notation, 3.33 can be reformulate as follows:

$$
\begin{aligned}
\frac{N(Y)}{Y} & =\frac{\left(A^{3}-B^{3}\right)(A-B)}{3 A^{2} B^{2}}-A B\left(\frac{1}{3 A^{2}}+\frac{1}{3 B^{2}}\right)+1 \\
& =\frac{(A-B)^{2}\left(A^{2}+A B+B^{2}\right)-A B\left(A^{2}+B^{2}\right)+3 A^{2} B^{2}}{3 A^{2} B^{2}} \\
& =\frac{(A-B)^{2}\left(A^{2}+A B+B^{2}\right)-A B(A-B)^{2}+A^{2} B^{2}}{3 A^{2} B^{2}} \\
& =\frac{(A-B)^{2}\left(A^{2}+B^{2}\right)+A^{2} B^{2}}{3 A^{2} B^{2}}>0
\end{aligned}
$$

With positive nominator and denominator, 3.32 is always positive. Furthermore, $N(0)=1$. Consequently, $N(Y)>0$ with $0<Y<1$. Therefore, $\frac{[F(X)]}{X}>0$. Since we have that $0<X<1$, the derivate of $F(X)$ is always positive in corresponding interval and $F(1)=\frac{\sqrt{3}}{2}$, we deduct that $0<\frac{Q^{*}}{Q_{j}^{*}}<\frac{\sqrt{3}}{2}$.

- if $\Delta=0, \frac{Q^{*}}{Q_{j}^{*}}=\frac{\sqrt{3}}{2} \sqrt{\frac{D[h+\alpha(a+p)]^{3}}{6 \alpha^{2} h^{2} s T}}=\frac{\sqrt{3}}{2}=0.86$

- if $\Delta<0, \frac{Q^{*}}{Q_{j}^{*}}=\frac{\sqrt{3}}{2 \cos \left\{\frac{1}{3} \arccos \left[\sqrt{\left.\frac{6 \alpha^{2} h^{2} s T}{[h+\alpha(a+p)]^{3} D}\right]}\right\}\right.}$ 
As we showed in the last paragraphs of proof for the proposition 3.4.1

$0<\frac{1}{3} \arccos \left(\sqrt{\frac{6 h^{2} s\left(1+\alpha r_{c}\right) \alpha^{2} T}{\left[h\left(1+\alpha r_{c}\right)+\alpha(a+p)\right]^{3} D}}\right)<\frac{\pi}{6}$

With $r_{c}=0,0<\frac{1}{3} \arccos \left(\sqrt{\frac{6 h^{2} s \alpha^{2} T}{[h+\alpha(a+p)]^{3} D}}\right)<\frac{\pi}{6}$

because of $0<\frac{6 \alpha^{2} h^{2} s T}{D[h+\alpha(a+p)]^{3}}<1$ when $\Delta<0$.

Therefore, $\frac{\sqrt{3}}{2}<\cos \left[\frac{1}{3} \arccos \left(\sqrt{\frac{6 h^{2} s \alpha^{2} T}{[h+\alpha(a+p)]^{3} D}}\right)\right]<1$. As a result, we obtain that $\frac{\sqrt{3}}{2}<\frac{Q^{*}}{Q_{j}^{*}}<1$

As a conclusion, we demonstrate that

$$
\begin{aligned}
& \text { — if } \Delta>0,0<\frac{Q^{*}}{Q_{j}^{*}}<\frac{\sqrt{3}}{2} . \\
& \text { — if } \Delta=0, \frac{Q^{*}}{Q_{j}^{*}}=\frac{\sqrt{3}}{2} \\
& \text { — if } \Delta<0, \frac{\sqrt{3}}{2}<\frac{Q^{*}}{Q_{j}^{*}}<1 .
\end{aligned}
$$

As a result, we can observe that our optimal quantity, $Q^{*}$ is always less than $Q_{j}^{*}$. Moreover, a significant difference between these optimal production quantities in the first two cases. Even in the third case, the difference is not negligible. Despite the simplicity of the traditional formula, this comparison demonstrates that it only provides a rough approximation of consequence of considering operation related financing cost on the optimal production quantity.

\subsection{Numerical analysis}

In this paragraph, a numerical example illustrates variations of different operation costs on the total cost, including the financial cost. Values of parameters are given in Table 3.3. In order to compare with the EOQ model, the sale unit price is not considered in this analysis. Additionally, we set the interest rate at $12 \%$ which is the weighted average cost of capital (WACC) used in the practice as stated in Serrano et al. (2017).

Table 3.3 - Parameter values for the numerical example

\begin{tabular}{ll}
\hline Parameter & Value \\
\hline Demand rate, $d$ & 2000 \\
Number of periods, $T$ & 12 \\
Purchasing unit cost, $a$ & 10 \\
Setup cost, $s$ & 20000 \\
Production unit cost, $p$ & 10 \\
Inventory holding unit cost, $h$ & 1 \\
Delay in payment from customer, $r_{c}$ & 1 \\
Delay in payment to supplier, $r_{f}$ & 1 \\
Interest rate, $\alpha$ & 0.12 \\
Sales unit price & not considered \\
\hline
\end{tabular}




\subsubsection{Comparison betweens results obtained by the proposed model and the classic EOQ model}

We first compute the optimal production quantity with the proposed model and the classic EOQ model. By applying the formula presented in Proposition 3.4.1, we obtain that $Q^{*}=5507$ for the values in Table 3.3. Moreover, the optimal quantity with the EOQ model is $Q_{E O Q}^{*}=\sqrt{\frac{2 D s}{h T}}=8944$. These results verify Proposition 3.4.3. In figure 3.5, we illustrate the total costs calculated by these two models. We denote that $C_{W C R}$ represents the total cost in $E O Q_{P(W C R)}$ model including the logistic cost $C L$ and financial cost $C F$ (i.e. $C_{W C R}=C L+C F$, as presented in equation $(3.16)$ ). $C_{E O Q}$ is the total cost in the EOQ model which is mathematically formulated as $C_{E O Q}=D(a+$ $p)+\frac{s D}{Q}+\frac{h T Q}{2}$ which is equal to $C L$ in $E O Q_{P(W C R)}$ model. Moreover, the optimal quantities are indicated in the figure which verifies the proposition 3.4 .3 .

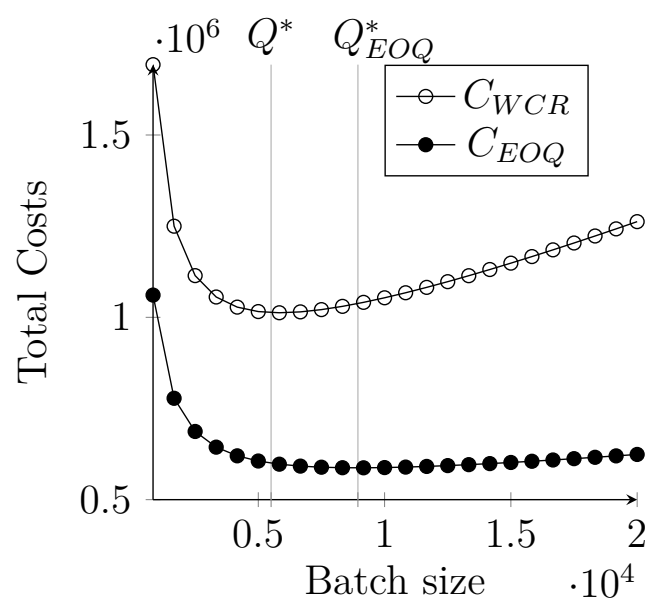

Figure 3.5 - Comparison of total cost between the EOQ and proposed model

Next, we measure the increase of total cost $(C L+C F)$ by using our proposed model in place of the EOQ model. To do so, we compare between the optimal total $\operatorname{cost} C_{W C R}^{*}$ with $Q^{*}$ and the total cost with $C_{W C R}^{*}\left(Q_{E O Q}^{*}\right)$ introducing $Q_{E O Q}^{*}$. As a result, we obtains that $\Delta C_{W C R}^{*}=$ $\frac{C_{W C R}^{*}\left(Q_{E O Q}^{*}\right)-C_{W C R}^{*}}{C_{W C R}^{*}}=2.5 \%$ which indicates a $2.5 \%$ increase in profit with our proposed model that considers the financing cost of OWCR versus the classic EOQ model.

\subsubsection{Illustration of the trade-off between costs}

As mentioned in Corollary 3.4.1.2, there exists a trade-off between the setup related costs and all other costs. In Figure 3.6, we illustrate the trade-off with the given instance. Knowing that only the terms in function of $Q$ are involved in the trade-off, the mathematical formulation of the setup related cost, $C_{s}$, and other costs, $C_{o}$ are thus presented as follows:

$$
\begin{aligned}
& C_{s}=\frac{\left(1+\alpha r_{c}\right) D s}{Q}=\frac{768000000}{Q} \\
& C_{o}=\frac{\alpha h T^{2}}{6 D} Q^{2}+\frac{h T+\alpha\left(h r_{c}+a+p\right) T}{2} Q=0.00012 Q^{2}+21.12 Q
\end{aligned}
$$




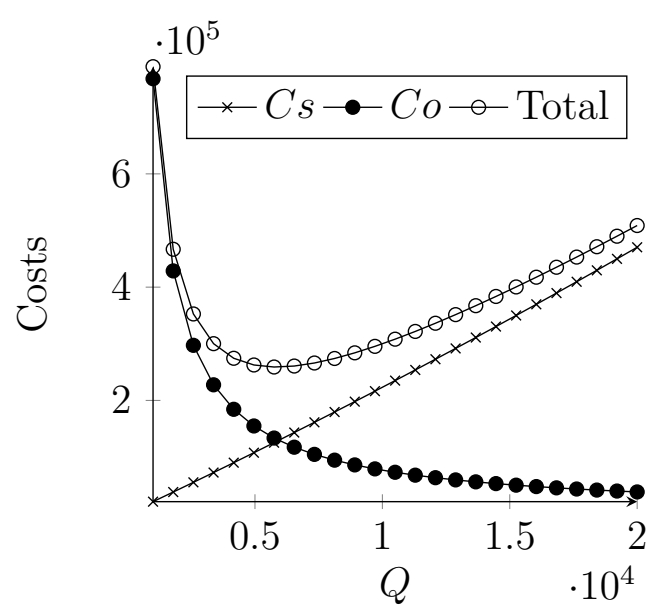

Figure 3.6 - Trade-off between batch size related costs

\subsubsection{Sensitivity of $Q^{*}$ to varying parameters in the classic and proposed $E O Q$ models}

In Figures 3.7 and 3.8, we illustrate the sensitivity of the optimal quantities of both models with varying setup cost and inventory holding costs as shown in Corollary 4.3.1. In these tests, we only consider the fixed setup cost and unit inventory holding cost which appear as parameters in both models. The purchasing and production unit costs are set to zero. As a result, we observe that the optimal batch size of the proposed model varies less than the one of the $E O Q$ model. By introducing the financial cost, the optimal production lot size of our propposed model is less sensitive than in the classic model to changes in $s$ and $h$ as we state in Corollary 3.4.3.1.



Figure 3.7 - Variations of optimal batch sizes to $s$ with both models

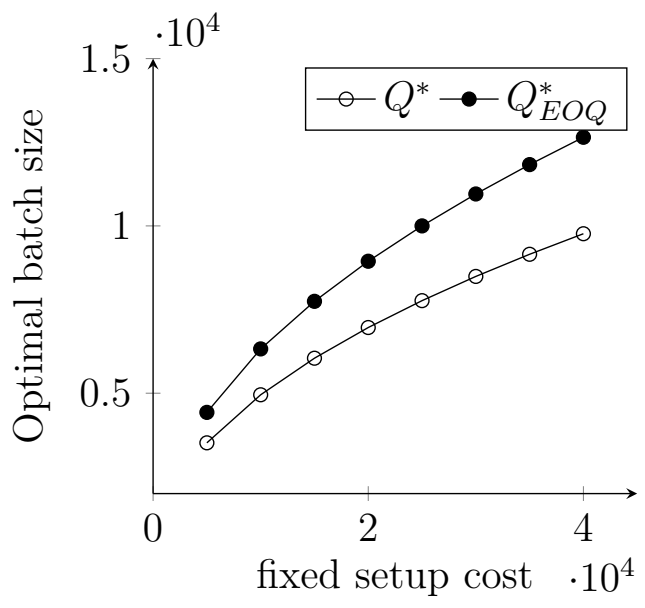

Figure 3.8 - Variations of optimal batch sizes with varying $s$ with the models

\subsection{Conclusions}

In this chapter, a new generic model of operations-related working capital requirements in the EOQ context is presented. Such a model allows us to measure the evolution of the WCR over the continuous-time planning horizon. Furthermore, an EOQ-based profit maximization model is developed considering the financing cost of the WCR. The analytical formula of the optimal EOQ is derived and associated analysis are provided to highlight some managerial insights. It is shown that 
an increase in purchasing, production unit costs or the interest rate reduces the optimal production lot size in order to obtain the maximum profit. Moreover, it is demonstrated that the economic quantity proposed by our model is always smaller than the optimal quantity of the traditional EOQ model. Thus, it is desirable to launch more frequent production with smaller lots in this joint management case in order to reduce inventory levels and their associated holding costs. Additionally, a comparison with the approximation of Jesse et al. (1983) demonstrates that our model provides a more coherent and accurate measure of the optimal production quantity for a profit maximization objective when considering financial aspects. Finally, the numerical example illustrates the reduced sensitivity of our model to several parameters when compared with the classic EOQ model.

As we reveal for this problem, there is a complex trade-off between the multitudes of different operational and financial costs in order to maximize the profit. For future research, we may first benefit from this trade-off to verify if the Zero-Inventory-Property is still valid in the dynamic lotsizing problem with time-varying demand. Second,Based on this trade-off, it could be interesting to establish heuristics for the capacitated lot-sizing problem considering OWCR financing costs. This is inspired by the way how the trade-off in the EOQ problem is used for the capacitated lot-sizing model proposed by Silver \& Meal (1973), the Least Unit Cost heuristic proposed by Gorham (1968) and the Part Period Balancing heuristic proposed by DeMatteis (1968). Lastly, it is shown that the delay in payment to the supplier has no influence on the optimal production quantity. However, it would be interesting to look into a two-echelon, supplier-customer relationship because, in this context, the delay in payment to supplier (accounts payable for the customer) would simultaneously influence the accounts receivable for the supplier. Thus, the customer's production policy would restrict the policy of the supplier and the delay in payment to supplier would no longer be negligible. 



\section{$-4$}

\section{Dynamic lot-sizing based discounted}

model considering the financing cost of working capital requirement

\subsection{Introduction}

In the first chapter, we take into consideration the financing cost of working capital requirements in the classic EOQ model in which the constant demand is assumed. To relax this assumption of constant demand to a more realistic time-varying demand, models of the dynamic lot-sizing problem (DLS) are proposed in the production planning literature. Hence, the objective here is to integrate the financing cost of WCR into the DLS models. We studies the case of Uncapacitated Lot-Sizing Problem (ULS) which is the case of single-level, single-product, constant parameter context and with infinite production capacity. First, a generic modeling of WCR is established in the ULS context that allows us to trace the evolution of WCR during the planning horizon. Second, a discounted cash flow model on the basis of the classic ULS model is built considering the financing cost of WCR. Subsequently, inspired by the classic Uncapacitated Lot-Sizing Problem (ULS), the Zero-InventoryOrdering (ZIO) property is proven valid in this problem. With possession of this property, a dynamic programming-based algorithm is constructed which resolves the problem in $O\left(T^{4}\right)$. A numerical analysis is effectuated that shows the difference between the classic ULS model and the proposed model in term of optimal production program. Moreover, the sensitivity analysis are performed with varying parameters.

\subsection{Dynamic lot sizing models}

After inquiring into the problem over continuous-time horizon with constant demand, the focus of this chapter is on the integration the financial cost of the WCR into the dynamic lot-sizing problem (DLS). In an Advanced Planning System (APS), DLS models are involved in the production planning as presented in Figure 2.4 of chapter 1. Thus, DLS models are close to the short term machine scheduling topic as well as sales planning as a Capable-To-Promise (CTP) logic (Fleischmann (2005)) to verify the fulfillment of client's demands. Additionally, supplier selection and capacity planning are frequently taken into consideration in DLS models and a large amount of literature is dedicated to the production planning problem by using DLS models. "This is due to the ubiquity of lot-sizing 
and scheduling decision problems in manufacturing firms and the large variety of production types in these firms, which often require specialized models" (Lang (2010)).

Early seminal work of Wagner \& Whitin (1958) is deemed as the initial effort to deal with this problem that extends the EOQ problem over discrete time horizon and handles time-varying demand. Similar to the EOQ problem, the DLS problem consist in determining production planning with setups periods and production quantities. The planned setup periods consequently derives the inventory holding level as well as the associated holding costs. Thus, the objective of DLS is to determine the best trade-off that minimizes the sum of production, setup and inventory holding costs. The main feature of the ULS models and the classification are given in Section 2.3.2. In the following paragraph, we will mainly focus on the ULS model.

\subsubsection{The uncapacitated Lot-Sizing (ULS) model and mathematical for- mulations}

As stated above, single-level, multi-product production planning problem with infinite production capacity can be solvable in polynomial time because it can be reduced to $N$ polynomial single production planning problems. Nevertheless, the problem becomes more difficult to handle with capacity constraints. NP-hardness is proven for both single- and multi-product cases with the production capacity in work of Bitran \& Yanasse (1982). In this chapter, we consider the financial cost of WCR in the single-site, single-product, big bucket and with infinite capacity case, i.e. the proposed model is based on $U L S$ model. The motivation of considering this case is mainly based on two points:

- With the above-presented classification, it is reasonable to start the study with the most basic problem.

- In studies of capacitated single-product or multi-product problems, the $U L S$ problem is often considered as a sub-problems to tackle. Examples can be found in the application of the Lagrangian relaxation (e.g. Thizy \& Van Wassenhove (1983)) to CLSP and column generation (Cattrysse et al. (1990)) to DLSP.

\section{ULS model and aggregate formulation}

The $U L S$ problem is described as follows and the associated formulation are given as well. First, the ULS model aims at satisfying time-varying demand over a planning horizon of $T$ periods and determining timing of setups and production lot size $Q_{t}$ in these periods. Such a planning should minimize the sum of the up-mentioned costs which includes:

- unit production cost $p_{t}$ (where $\mathrm{t}=1, . ., \mathrm{T}$ is the period);

- setup cost $s_{t}$, a fixed cost incurred when a production is planned in a period $t$,

- unit inventory holding cost $h_{t}$ per period.

The assumptions of this problem are:

- No replenishment and production delays as they are negligible compared to the period duration;

- Demand should be met on time (no backlogging);

- Only inventory of final product is considered;

- Initial and final stocks are set to zero;

- One unit of finished product is manufactured using one unit of raw material.

In the DLS literature, Mixed Integer Programming (MIP) formulation is not directly adopted for solving the $U L S$ problem as the case of Wagner \& Whitin (1958) and as stated by Brahimi et al. (2017). However, as above-mentioned, the $U L S$ problem is often considered as a subproblem of more complex DLS problem for which the MIP formulation is built for solution procedure. In the following, different MIP formulations of the ULS problem are presented in the following paragraphs and the associated parameters are summarized below. 


\begin{tabular}{ll}
\hline Parameter & Definition \\
\hline$T$ & Horizon length \\
$d_{t}$ & Demand in period $t$ \\
$d_{t k}$ & Sum of demands from period $t$ to period $k$ \\
$h_{t}$ & Unit inventory cost in period $t$ \\
$p_{t}$ & Unit production cost in period $t$ \\
$s_{t}$ & Fixed setup cost in period $t$ \\
\hline
\end{tabular}

Table 4.1 - Parameters

\begin{tabular}{ll}
\hline Variable & Definition \\
\hline$Q_{t}$ & Total production quantity in period $t$ \\
$X_{t k}$ & Production quantity in period $t$ for satisfying (a part of) demand in period $k$ \\
$I_{t}$ & Product quantity held in inventory in period $t$ \\
$Y_{t}$ & Binary variable which indicates whether a setup occurs in period $t$ or not \\
\hline
\end{tabular}

Table 4.2 - Decision variables

A natural formulation of the ULS problem is the aggregate formulation provided as below. In this formulation, products manufactured in a same period $t$ are aggregated as $Q_{t}$ which aims to satisfy demand(s) of following period(s).

$$
\begin{array}{ccc}
\operatorname{Min} & \sum_{t=1}^{T}\left(s_{t} Y_{t}+p_{t} Q_{t}+h_{t} I_{t}\right) & \\
\text { s.t. } & I_{t-1}+Q_{t}=d_{t}+I_{t} & \forall t \\
& d_{t T} Y_{t}-Q_{t} \geq 0 & \forall t \\
Q_{t}, I_{t} \geq 0 & \forall t \\
Y_{t}=0 \text { or } 1 & \forall t
\end{array}
$$

The objective function (4.1) minimizes the sum of setup, production and inventory holding costs over the entire horizon. Constraints (4.2) are the inventory balance equations. They express that the entering stock $I_{t-1}$ added to the current period production $Q_{t}$ is used to satisfy the demand $d_{t}$ and the remains is kept in stock at the end of the period $I_{t}$. Constraints (4.3) ensure that a setup only occurs when a production planned in period $t$. Constraints (4.4) and (4.5) relate the continuous production variables $Q_{t}$ to the binary setup variables $Y_{t}$.

\section{Reformulations of the ULS model}

Three reformulations of the ULS model are presented in this section. The first two reformulations are based on the fact that:

- Constraints 4.2 balance the inventory by the difference between the remaining in the inventory plus the new production and the consumption of inventory for satisfying demands. The first reformulation combines the unit production costs with the holding costs in order to replace the variable of inventory quantity.

- The second reformulation disaggregates the production variable, $Q_{t}$ because it merely represents all products manufactured in a certain period without knowing for which demand they are produced. Therefore, a disaggregate formulation can be formulated, namely, the facility location-based (FAL) formulation. 
Formulation without inventory variable (NIF):

$$
\begin{aligned}
& \operatorname{Min} \sum_{t=1}^{T} s_{t} Y_{t}+\sum_{t=1}^{T}\left(p_{t}+\sum_{i=t}^{T} h_{i}\right) Q_{t}-\sum_{t=1}^{T} h_{t} d_{1 t} \\
& \text { s.t. } \quad \sum_{t=1}^{T} Q_{t}=d_{1 T} \\
& \sum_{t=1}^{t} Q_{i} \geq d_{1 t} \quad t=1, \ldots, T-1 \\
& Q_{t} \leq d_{t T} Y_{t} \quad \forall t \\
& Q_{t} \geq 0, Y_{t}=0 \text { or } 1 \quad \forall t
\end{aligned}
$$

\section{Facility location-based formulation (FAL):}

In this disaggregate formulation, let us define $X_{t k}$ as the quantity of production in the period $t$ for the demand of period $k$. Moreover, $c_{t k}=p_{t}+h_{t}+h_{t+1}+h_{t+2}+\ldots++h_{t-2}+h_{t-1}$. The formulation can be written as:

$$
\begin{array}{ccc}
\operatorname{Min} & \sum_{t=1}^{T}\left(s_{t} Y_{t}+\sum_{i=t}^{T} c_{t i} X_{t i}\right) & \\
\text { s.t. } & \sum_{i=1}^{T} X_{i t}=d_{t} & \forall t \\
& d_{k} Y_{t}-X_{t k} \geq 0 & \forall t, k \\
X_{t k} \geq 0 & \forall t, k \\
Y_{t}=0 \text { or } 1 & \forall t
\end{array}
$$

In an equivalent plant location formulation, Krarup \& Bilde (1977) demonstrate that the linear programming relaxation of this formulation has an optimal solution with an integer value of the variable $Y$. The authors also propose an $\mathrm{O}\left(T^{2}\right)$ algorithm to solve the problem. Wagelmans et al. (1992) improve the complexity to $\mathrm{O}(T \log T)$ based on this formulation. The main idea of the algorithm is to identify periods that will never be used for production in an optimal plan.

\section{Shortest path formulation (SHP)}

In addition to former formulations, Evans (1985) provides a graph-based shortest path formulation (SHP). In the graph representation, nodes represent the production periods including a dummy period at the end of the planning horizon, $T+1$. Arcs that link each pair of nodes are also found in the graph. An arc between node $t$ and $k$ signifies a production option of full amount of demands between the periods $t$ and $k-1$ at the period $t$. Therefore, the objective is to find the shortest path from the first node to the last one. To do so, the associated production related costs are allocated to each arc. Let $E_{t k}$ be the total cost of sub-plan from period $t$ to $k-1$ including setup, production and inventory holding costs for demands $d_{t k}=d_{t}+d_{t+1}+\ldots+d_{k-1}, E_{t k}=s_{t}+p_{t} d_{t k}+\sum_{i=t+1}^{k-1} h_{i-1} d_{i k}$. Let $F_{t k}$ be the decision variable. If $F_{t k}=1$, the total demand from period $t$ to period $k-1$ (i.e., $d_{t k}$ ) is produced in period $t$, and 0 otherwise. 


$$
\begin{array}{cll}
\operatorname{Min} & \sum_{t=1}^{T}\left(s_{t} Y_{t}+\sum_{k=t+1}^{T+1} E_{t k} F_{t k}\right) & \\
\text { s.t. } & \sum_{i=1}^{T} F_{1 i}=1 & \\
\sum_{i=1}^{t-1} F_{i t}=\sum_{i=t+1}^{T+1} F_{t i} & t=2, \ldots, T \\
\sum_{i=t+1}^{T+1} F_{t i} \leq Y_{t} & \forall t \\
F_{t k} \geq 0 & \forall t, k \\
Y_{t}=0 \text { or } 1 & \forall t
\end{array}
$$

Constraints 4.17) ensure that no more than one arc outgoing from the node 1. Constraints 4.18 ensure that the flow balance is not violated at nodes $2, \ldots, T+1$. Constraints $(4.19)$ force the setup binary variable $Y_{t}$ to be one whenever there is production in period $t$. The linear relaxation of this formulation has an optimal solution with integer variables $Y$ (constraints (4.21)).

A comparison of the last two formulations is given in work of Gao et al. (2008) on the effectiveness of solving MIP formulation with tight LP relaxations by establishing specialized primal-dual algorithms . A research timeline with different formulations is also provided.

\section{Solution procedure}

The first exact algorithm for this problem has been proposed by Wagner \& Whitin (1958) based on dynamic programming (DP) which solve the problem in $\mathrm{O}\left(T^{2}\right)$. This kind of method explores the solution space of a problem by implicit enumeration to find its optimal solution. The authors proved a strong structural property of the optimal planning.

Theorem 4.2.1. There exists an optimal solution with $Q_{t} I_{t-1}=0$ for all $t$, where $I_{t}$ represents the inventory level, which means the production will only occur with no product held in inventory.

Using dynamic programming with the above property, the search space can be significantly reduced to at most $\frac{T(T+1)}{2}$. Another interpretation of this property is, in the optimal planning, the production quantities can only be the sum of demand(s) in following consecutive period(s). In this literature, this problem draw many attention as studied in Zabel (1964), Eppen et al. (1969) and Lundin \& Morton (1975). However, the complexity of the WW algorithm is further improved by respective efforts of Wagelmans et al. (1992), Aggarwal \& Park (1993) and Federgruen \& Tzur (1991). Reviewing literature of this problem, we notice that the DP technique has been largely considered which will be further discussed in the next section.

\subsection{The discounted cash flow model of the DLS problem}

The DLS models are always built based on given or estimated demand for short-term or mediumterm production planning problems. Thus, in the lot-sizing literature, the traditional planning model implicitly assumes that all the investment is financed by equity without debt and the financial budget limitations in expansions for incapacity are often neglected. It is rarely the case in real world because operational and financial decisions are interrelated for reaching the maximum profitability and performance of the company in an extremely competitive economic context. Consequently, a company may raise its capital from profits, external loans, or bonds and the financial decisions of strategic capacity development are usually set for a large planning horizon. Therefore, the time value of money should be considered for its significant impact on investment decision. 
However, the time value of capital is generally not taken into account or sometimes assumed that all (variable) costs and/or revenues are discounted, as in the work of Karabuk \& Wu (2003) and Claro \& De Sousa (2012). More similar case can be found in the review of Martínez-Costa et al. (2014). To the best of our knowledge, since 1990s, discounted cash flow (DCF) models have appeared in which the time value of money is considered using a discount factor or cost of capital. The formulation of Helber (1998), presented below, is considered as one of these early efforts introducing the DCF model into a lot-sizing problem. The authors treated the multi-level DLS problem under multi-capacity constraints and setup times in case of multi-level CLSP. In this work, the impact of discounted cash-flow on lot-sizing decision within MRP II context is analyzed. Furthermore, in Table 4.4, a comparison between the related works and the model developed in this chapter is provided.

\subsubsection{Mathematical Formulation of the discounted cash flow model for DSL problems}

Parameter and decision variable notations are presented in Table 4.3. In this formulation, the setup and production costs are constant over time.

\begin{tabular}{ll}
\hline Parameter & Definition \\
\hline$a_{i j}$ & number of units of item $i$ required to produce one unit of item $j$ \\
$b_{m t}$ & available capacity of resource $m$ in period $t$ \\
$\alpha$ & interest rate on a perfect capital market \\
$B$ & Big number \\
$d_{i t}$ & external demand of item $i$ in period $t$ \\
$E$ & number of items $i$ \\
$K_{m}$ & set of items that require resource $m$ \\
$M$ & number of resources $m$ \\
$P_{i}$ & set of predecessors of item $i$ (immediate or other) \\
$p s_{t}$ & direct expense related to a setup for item $i$ \\
$p o_{t}$ & direct expense related to an operation for item $i$ \\
$Q_{i t}$ & production quantity (lot-size) of item $i$ in period $t$ \\
$S_{i}$ & set of immediate successors of item $i$ \\
$T$ & number of period $t$ \\
$t o_{i}$ & operation (processing) time per unit if item $i$ \\
$t s_{i}$ & setup time of item $i$ \\
$Y_{i t}$ & binary setup decision variable of item $i$ in period $t$ \\
$I_{i t}$ & planned end-of-period inventory of item $i$ in period $t$ \\
\hline
\end{tabular}

Table 4.3 - Parameters

The DCF based multi-level CLSP model in Helber (1998) is written as: 


$$
\begin{array}{cll}
\text { Min } \quad Z=\sum_{t=1}^{T} \sum_{i=1}^{E}\left(p s_{i} Y_{i t}+p o_{i} Q_{i t}\right) \frac{1}{1+\alpha^{t}} & \\
\text { s.t. } I_{i, t-1}+Q_{i t}-\sum_{\substack{j \in S_{i} \\
t+1 \leq T}} a_{i j} Q_{j, t+1}-I_{i t}=d_{i t} & \forall i, t \\
\sum_{i \in K_{m}}\left(t s_{i} Y_{i t}+t o_{i} Q_{i t}\right) \leq b_{m t} & \forall m, t \\
Q_{i t}-B Y_{i t} \leq 0 & \forall i . t \\
Q_{i, 1}=0 & \forall i \text { with } P_{i} \neq \emptyset \\
Q_{i t}, I_{i t} \geq 0 & \forall i, t \\
Y_{i t}=0 \text { or } 1 & \forall i, t
\end{array}
$$

The objective function 4.22 minimizes the NPV of all payments for setup and productions. The constraints (4.23) are for the inventory balance between periods. (4.24) are the capacity constraints. Constraints (4.25) prohibit setup occurs in a period with no production. (4.26) reflect the assumption of no initial inventory for all items. (4.27) are for non-negativity of decision variables. (4.28) ensure the setup variables are binary.

\subsubsection{Literature review of discounted cash flow model for the DLS prob- lem}

In the literature of DLS problems, the time of value money (TVM) is often considered (among others, e.g. corporate tax) in order to take into account economic impact on operational decision. TVM measures the net present value (NPV) of future cash flows and reflects the financial impact of logistic cost in the operating cycle over time. As mentioned in the above paragraphs, since 1990s, this financial aspect has appeared in DLS models. To do so, either the associated costs are assumed discounted, or a discount rate is adopted as presented in the formulation of Helber (1998). Similar concepts can also be found in others works ever since. Papageorgiou et al. (2001) applied the MIP model to facilitate the strategic supply chain decision-making process in the context of pharmaceutical industries. The authors adopted a discount factor associated with the inflation rate. Levis \& Papageorgiou (2004) extended the previous work by determining both product portfolio and multi-site capacity planning in case of uncertain clinical trials. Fleischmann et al. (2006) built a strategic-planning model to optimize the allocation of various product to BMW's global production sites in an annual planning horizon. In the MIP model, the authors determined the investment decision for production related operations considering the financial impact on cash flow with NPV calculations. Wang et al. (2007a) addressed acquisition and allocation of LCD (Liquid-Crystal Display) manufacturing industries which requires a high amount of capital investments. In this problem, the authors included not only constraints of operational aspect, e.g., resource capacity constraints and backorder, but also financial aspects of finite budget and the time value of capital and asset. Wang et al. (2007b) proposed a mathematical programming model for a generalized resource portfolio problem in context of semiconductor testing industry considering the financial resource constraints and the time value of capital. Bihlmaier et al. (2009) proposed a mathematical formulation to deal with consecutively strategic decisions and both tactical and operational decisions in order to optimize the NPV of profits over a long planning horizon. The authors showed the strength of their solution procedure based on Benders' Decomposition which significantly reduce the solution time and is promising for an application of a real world scenario. Yang et al. (2009) investigated simultaneously task assignment, resource allocation and equipment acquisition/disposal decision within a scenario based-resource portfolio model. Thomas \& Bollapragada (2010) developed an analytical decision tool based on a capacity planning model to estimate product costs and demands in order 
to maximize the NPV of profit for General Electric. More related works are referred in You et al. (2010), Lim et al. (2013a), Lim et al. (2013b) and Mitra et al. (2014) in various industry contexts. A comparison between these works and our research is presented in Table 4.4 .

\begin{tabular}{|c|c|c|c|c|c|c|c|c|}
\hline Authors & $\begin{array}{c}\text { SC } \\
\text { structure }^{1}\end{array}$ & $\begin{array}{c}\text { Nb of } \\
\text { products }^{2}\end{array}$ & $\begin{array}{l}\text { Finite } \\
\text { budget }\end{array}$ & $\begin{array}{l}\text { Time value } \\
\text { of money }\end{array}$ & Inflation & $\begin{array}{l}\text { Delay of } \\
\text { payment }\end{array}$ & WCR & Objective $^{3}$ \\
\hline Helber (1998) & $\mathrm{S}$ & $\mathrm{M}$ & & $\sqrt{ }$ & & & & MinC \\
\hline Papageorgiou et al. (2001) & S & M & & $\sqrt{ }$ & $\sqrt{ }$ & & & MaxP \\
\hline Levis \& Papageorgiou (2004) & M & M & & $\sqrt{ }$ & $\sqrt{ }$ & & & MaxP \\
\hline Fleischmann et al. (2006) & M & M & $\sqrt{ }$ & $\sqrt{ }$ & & & & $\mathrm{MinC}$ \\
\hline Wang et al. (2007a) & $\mathrm{S}$ & M & $\sqrt{ }$ & $\sqrt{ }$ & & & & MaxP \\
\hline Wang et al. (2007b) & $\mathrm{S}$ & M & $\sqrt{ }$ & $\sqrt{ }$ & & & & MaxP \\
\hline Bihlmaier et al. (2009) & M & M & & $\sqrt{ }$ & & & & MinC \\
\hline Yang et al. (2009) & $\mathrm{S}$ & M & $\sqrt{ }$ & $\sqrt{ }$ & & & & MaxP \\
\hline Thomas \& Bollapragada (2010) & $\mathrm{S}$ & & $\sqrt{ }$ & $\sqrt{ }$ & & & & MaxP \\
\hline You et al. (2010) & M & M & & $\sqrt{ }$ & & & & $\mathrm{MaxP}$ \\
\hline Lim et al. (2013a) & $\mathrm{S}$ & M & & $\sqrt{ }$ & & & & MaxP \\
\hline Lim et al. (2013b) & M & M & & $\sqrt{ }$ & & & & $\operatorname{MaxP}$ \\
\hline Mitra et al. (2014) & $\mathrm{S}$ & M & & $\sqrt{ }$ & & & & MinC \\
\hline This work & $\mathrm{S}$ & $\mathrm{S}$ & & $\sqrt{ }$ & $\sqrt{ }$ & $\sqrt{ }$ & $\sqrt{ }$ & $\operatorname{MaxP}$ \\
\hline
\end{tabular}

Table 4.4 - Brief literature review

In the context of the project RCSM, we investigate the consolidation of financial needs of all actors of supply chain. We assume the budget is no longer a constraint. Therefore, we rather consider the integration of the financing cost of WCR in the ULS model. We choose the profit maximization objective because the WCR is presumed covered by the cost part of sales revenue. The detailed explanation is given in the following section. Compared to the related literature, we consequently take into account the delay in payment because the WCR is generated by the timing mismatch between receivables and payables.

\subsection{ULS-based profit maximization DCF model}

\subsubsection{OWCR modeling}

The discounted cash flows method explicitly takes into account the time difference between the cash flows but it does not take into account the financial borrowing costs because, in traditional models, these costs have supposedly no influence on thee optimal operational decision. As we challenge this assumption, we want to integrate only the financial costs linked to operational decisions in the cash flows (i.e., financing cost of OWCR). To do so, we propose a generic OWCR formulation in a tactical planning context. In this formulation, both the delay in payment to supplier and from client are taken into account and the time value of money is considered in order to calculate present values of incoming and outgoing cash flows. Furthermore, the level of inventory should be able to be precisely measured over the planning horizon with this OWCR formulation as inventory holding costs make up a substantial portion of the OWCR.

Therefore, we adopt the cash to cash cycle methodology presented in Theodore Farris \& Hutchison (2002) and Hofmann \& Kotzab (2010) where the OWCR for producing a unit of product depends on the amount invested into the related operations (e.g., purchasing, setup, production and inventory holding) and the number of periods to finance these investments before recovering from corresponding sales. However, we firstly consider that the profit portion of the sales revenue are not available to cover the OWCR. This is because the profit margin of the company does not correspond to the definition of 
working capital requirement since it has never been required. Furthermore, this assumption supposes that the company does not prioritize its profit allocation to WCR financing. Profit can be allocated to any of a number of objectives for the firm including debt reduction, internal or external investment, or dividend payments. Our model is a partial model of the company and we do not assume that allocation of profit to WCR financing rather than to other objectives (e.g., new investments) is optimal. Moreover, following the Modigliani Miller Theorem, a company should not affect a specific financial resource (here profit) to a specific object (here WCR financing). In consequence, we adopt the scheme that the WCR generated by producing a product is only effectively recovered when that product is sold.

This assumption complicates the calculation of the financing duration of setup costs because it is paid in its entirety at a single point in time for the entire production lot. Thus, we must progressively and uniformly recover the setup cost from the sales revenue of all products in the same production lot. Therefore, these products share the fixed setup cost equally as the invested amount and the number of periods to finance is the time difference between the period where the setup occurs and the period of sale of each product.

\subsubsection{Assumptions}

Since this model is built in context of the ULS (Uncapacitated Lot-Sizing) problem, the OWCR formulation and the corresponding profit maximization model proposed in this section are under the following assumptions:

- Production:

- No replenishment and production delays as they are negligible compared to period duration;

- Demand should be met on time (no backlogging);

- Only inventory of final product is considered;

- Initial and final stocks are defined as zero;

- One unit of finished product is manufactured using one unit of raw material.

- Financial:

- All logistic and financial costs are paid in the beginning of periods;

- The profit from selling products is not used for financing the OWCR;

- The fixed setup cost of a given lot is progressively and uniformly recovered over the different periods during which the products of the corresponding lot are sold;

- All holding costs paid for a product for all periods it remains in inventory are not recovered until its sale.

\subsubsection{Physical and financial flows illustration}

With the aforementioned assumptions, we express in detail, in Figure 4.1, the physical and financial flows generated by a production in period 1 for the demand of period 3 . In this case, we assume the delays in payment from client and to supplier are both one period.

- Physical flows are presented in the first bloc. They are generated by the decision made in the operating cycle, such as purchase, production, inventory holding and delivery. Therefore, the physical flows link all decisions in the graphic with the single line arrow.

- As financial flow, first, the logistic costs are immediately generated by the physical activities presented by small squares in the second bloc. Meanwhile, the WCR financing costs are also to be paid from the same period of corresponding logistic costs until the reception of revenue. These financial costs are presented with the double line arrow. 

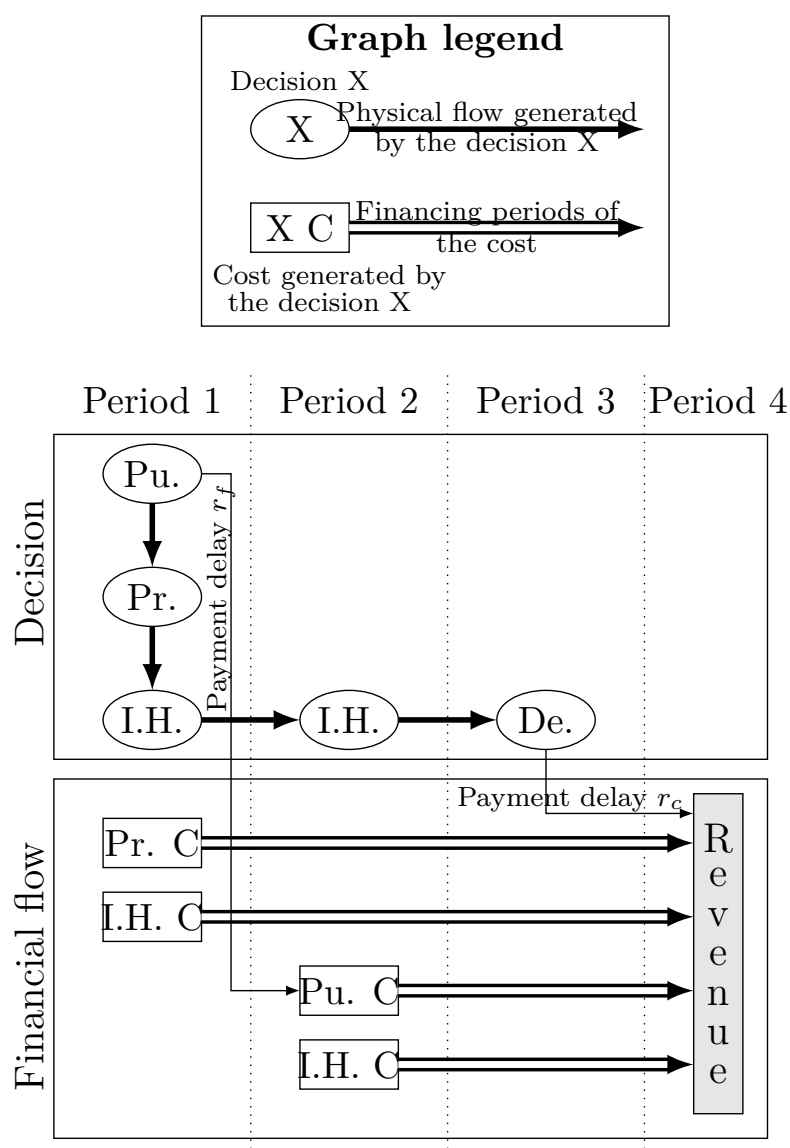

Pu. (Purchasing)/Pr. (Production)/I.H. (Inventory holding)/De. (Delivery)

Figure 4.1 - Physical and financial flows illustration 


\subsubsection{Parameters et decision variables}

In this model, parameters and decision variables are adopted for the single-site, single-level, singleproduct with infinite capacity case. They are respectively given in Tables 4.5 and 4.6. We assume these parameters are all nonnegative. We choose the decision variables of FAL (FAcility Location) formulation of the ULS. These disaggregated variables allow us to measure the exact amount of incoming and outgoing payments for all production related operations considered for calculating the OWCR.

\begin{tabular}{ll}
\hline Parameter & Definition \\
\hline$T$ & Horizon length \\
$d_{t}$ & Demand in period $t$ \\
$v$ & Unit product price \\
$h$ & Unit inventory cost per period \\
$p$ & Unit production cost \\
$s$ & Fixed setup cost \\
$a$ & Unit raw material cost \\
$r_{c}$ & Delay in payment from client \\
$r_{f}$ & Delay in payment to supplier \\
$\alpha$ & Discount rate per period \\
$\beta$ & Interest rate for financing OWCR per period \\
\hline
\end{tabular}

Table 4.5 - Parameters for OWCR modeling

\begin{tabular}{ll}
\hline Variable & Definition \\
\hline$Q_{t}$ & Total production quantity in period $t$ \\
$X_{t k}$ & Production quantity in period $t$ for satisfying (a part of) demand in period $k$ \\
$I_{t}$ & Product quantity held in inventory in period $t$ \\
$Y_{t}$ & Binary variable which indicates whether a setup occurs in period $t$ or not \\
\hline
\end{tabular}

Table 4.6 - Decision variables for OWCR modeling

\subsubsection{Mathematical formulation}

In our problem, OWCR includes all financial needs for the operations of purchasing, setup, production and inventory holding for satisfying a known, but variable demand in an operation cycle. The financial need of each $X_{t k}$ is modeled as the product of corresponding operation cost and the number of periods to finance.

- The operation cost is function of the operation unit cost and the value of $X_{t k}$. All unit costs of purchasing raw material, production and inventory holding are given as parameters. However, all products in a lot, $Q_{t}$, share the setup cost, $s$. Therefore, the setup unit cost per product is $\frac{s}{Q_{t}}$. However, when $Q_{t}=0$, it raises a problem of singularity. Therefore, it is further reformulated as $\frac{s Y_{t}}{Q_{t}+\left(1-Y_{t}\right)}$ to prevent the problem. Consequently, in case of $Q_{t}=0$, $Y_{t}$ will thus be zero. The denominator will be equal to one. In the other case, if $Q_{t}>0$, then $Y_{t}=1$ which leads that $\frac{s Y_{t}}{Q_{t}+\left(1-Y_{t}\right)}=\frac{s}{Q_{t}}$. Nevertheless, due to this non-linear formulation, we should prove the Zero-Inventory-Ordering (ZIO) property of this model to establish an effective resolution algorithm.

- The number of periods to finance depends firstly on the difference between $k$ and $t$. Furthermore, the mismatch between payment to supplier and payment from client may extend the number of periods of the horizon beyond that last demand for financing the production 
related costs. The delays in payment (i.e., $r_{c}$ and $r_{f}$ ) are introduced as parameters in order to quantify this mismatch. With these parameters, we define the beginning period to finance the corresponding production related cost as well as the end period as presented in table 4.7 .

\begin{tabular}{|c|c|c|c|c|c|c|c|}
\hline \multirow[b]{2}{*}{ Operations } & \multicolumn{3}{|c|}{ Operation cost } & \multicolumn{4}{|c|}{ Periods to finance } \\
\hline & Unit cost & $\times$ & Quantity & from & $\left(\begin{array}{c}\text { the period of } \\
\text { outgoing payment }\end{array}\right)$ & until & $\left(\begin{array}{c}\text { the period of } \\
\text { incoming payment }\end{array}\right)$ \\
\hline Purchasing & $\mathrm{a}$ & $x$ & $X_{t k}$ & from & $\left(t+r_{f}\right)$ & until & $\left(k+r_{c}-1\right)$ \\
\hline Setup & $\frac{s Y_{t}}{Q_{t}+\left(1-Y_{t}\right)}$ & $\times$ & $X_{t k}$ & from & $t$ & until & $\left(k+r_{c}-1\right)$ \\
\hline Production & $\mathrm{p}$ & $x$ & $X_{t k}$ & from & $t$ & until & $\left(k+r_{c}-1\right)$ \\
\hline Inventory holding & $\mathrm{h}$ & $x$ & $X_{t k}$ & & ancins & eriods & FP) \\
\hline
\end{tabular}

Table 4.7 - Formulation of OWCR components

Although the costs of purchasing, set-up and production are all one-time payments for each production lot, the inventory holding cost is regularly paid in all periods where the stock is held in inventory. For this reason, there is a cumulative effect of financing the inventory holding costs. Assuming that a production lot covers all demands from period $t$ to period $k$, which means that the production occurs in period $t$ at the latest. A unit of product of the demand in period $k$ will be sold in period $k$ and the client's payment will arrive in period $k+r_{c}$. Therefore, we should pay the inventory holding cost for this item from period $t$ until period $k-1$ (noted as "OWC $R_{\text {inv }}$ series"). The first of these payments occurs in period $t$ and must be financed until period $k+r_{c}-1$ (before the arrival of client's payment, so $k+r_{c}-t$ periods have to be financed in total.). The second is paid in period $t+1$ until the same period of $k+r c-1$ (in total, $k+r_{c}-(t+1)$ periods to finance.), etc. Consequently, for each $X_{t k}$, the financing period is $\frac{1+(k-t)}{2}(k-t)+r_{c}(k-t)$ periods including all periods to finance before and after the demand delivery.

Following this formulation of OWCR, the OWCR at the period $t$, noted $O_{t}$, can be expressed as follows:

$$
\begin{aligned}
O_{t}= & a \sum_{j=1}^{t-r_{f}} \sum_{l=t-r_{c}+1}^{T} X_{j, l}+p \sum_{j=1}^{t} \sum_{l=t-r_{c}+1}^{T} X_{j, l}+s \sum_{j=1}^{t} \sum_{l=t-r_{c}+1}^{T} \frac{Y_{j}}{Q_{j}+\left(1-Y_{j}\right)} X_{j, l} \\
& +h \sum_{j=1}^{t} \sum_{l=t-r_{c}+1}^{T} X_{j, l}(\min \{l, t+1\}-j)
\end{aligned}
$$

The formulation $\sum_{j=1}^{t-r_{f}} \sum_{l=t-r_{c}-1}^{T} X_{j, l}$ represents all products for which we still need to finance their production related operations at period $t$ considering the delays in payment. Especially for measuring the total amount of inventory holding cost to finance at period $t$, we need to count the number of completed payments in the $O W C R_{i n v}$ series until period $t$ for each $X_{j, l}$. However, we must consider whether we have sold the products of $X_{j, l}$ or not before reception of the client's payment (in other words, if $X_{t k}$ is still in inventory or not). $\min \{l, t+1\}-j$ precisely represents the total number of all payments for these two possible cases. The difference between these two cases as presented in formulation 4.29 is :

- If we have sold $X_{t k}$ at period $t$ (i.e. $l \leq t<l+r_{c}$ ), then the total amount to finance for inventory holding will not change during the periods before the arrival of client's payment. This is because we no longer need to pay the inventory holding cost for $X_{t k}$ in theses periods. Thus, no additional inventory holding cost must be financed which means the total amount is a function of $l-j$.

- On the contrary, if the $X_{t k}$ is still in inventory (i.e. $j \leq t<l$ ), then the total amount of inventory holding costs to finance depends on $t+1-j$. 
This formulation of OWCR allows us to follow the variation of OWCR over time in the planning horizon. It will be used in the following section for calculating the financing cost of OWCR.

\subsubsection{Objective function}

The objective of this problem is to maximize the NPV of the profit (denoted by Profit) by satisfying variable demand. To simplify the problem, the NPV of profit is defined as the difference between the NPV of revenue and the NPV of expenses. In our model, the revenue per period is a function of constant unit sales prices and demand quantity. The expenses include the production costs and the OWCR financing costs. The production cost is the sum of the costs for raw material purchasing, machine setup, production and inventory holding. The OWCR financing cost depends on the interest rate and the OWCR for satisfying all the demand on-time. Considering the delays of payment, the planning horizon must be extended to the period where the last incoming or outgoing cash flow is occurred. Mathematically, this accounting horizon is presented as $T+\max \left\{r_{f}, r_{c}\right\}$. For reason of simplification, $T$ denotes the accounting horizon in this section. Since the time value of money is considered in this model, both cash inflow (i.e., revenues) and outflow (i.e., expenses) are presented in NPV as follows:

- NPV of revenue in period $t, R_{t}$ : Because of the delay of the client's payment, we only receive the client's payment of demand of period $t-r_{c}$ in period $t$. Thus, $R_{t}=\frac{1}{(1+\alpha)^{t}} v D_{t-r_{c}}$.

- NPV of logistic cost in period $t, L C_{t}$ : All expenses of the production related operations must be immediately paid when they occur except for the purchasing cost which can be delayed $r_{f}$ periods according to the supplier agreement. Therefore, $L C_{t}=\frac{1}{(1+\alpha)^{t}}\left(a Q_{t-r_{f}}+\right.$ $\left.p Q_{t}+s Y_{t}+h I_{t}\right)$ with $Q_{t}=\sum_{k=t}^{T} X_{t k}$ and $I_{t}=\sum_{l=1}^{t} \sum_{k=t+1}^{T} X_{l k}$.

- NPV of OWCR financing cost in period $t, F C_{t}$ : The OWCR financing cost only depends on the interest rate per period and the amount of financial need (i.e., $O_{t}$ ) to cover in period $t . F C_{t}=\frac{1}{(1+\alpha)^{t}} \beta O_{t}$.

By summing over all periods of the accounting horizon, the objective function is formulated as follows :

$$
\text { Profit }=\sum_{t=1}^{T}\left(R_{t}-L C_{t}-F C_{t}\right)
$$

\subsubsection{ULS $S_{P(W C R)}$ model}

A mixed-integer model of the profit maximization problem, $U L S_{P(W C R)}$, is formulated as follows

$$
\begin{array}{ccl}
\text { Max } & \text { Profit } & \\
\text { s.t. } & Q_{t}=\sum_{k=t}^{T} X_{t k} & \forall t \\
& d_{k} Y_{t}-X_{t k} \geq 0 & \forall t, k \\
& Y_{t}-Q_{t} \leq 0 & \forall t \\
& \sum_{t=1}^{k} X_{t k}=d_{k} & \forall k \\
X_{t k} \geq 0, Y_{t} \in\{0,1\} & \forall t, k
\end{array}
$$

The objective function is to maximize the NPV of total profit (4.30). Then, constraints are similar to those of the FAL formulation of the ULS. Constraints (4.31) indicate that $Q_{t}$ is the total quantity of production that occurs in period $t$. Constraints 4.32 ensure that a setup is executed before production. Constraints (4.33) prevent a setup from occurring in a period with no production. Finally, constraints (4.34) assure all demands are satisfied. 


\subsection{Solution method}

In order to solve effectively the $U L S_{P(W C R)}$ problem, we propose an algorithm which avoids the difficulty of the non-linear formulation of the setup cost in the OWCR calculation. This algorithm is similar to the algorithm which is based on the Zero-Inventory-Ordering (ZIO) property established for the ULS problem with infinite capacity by Wagner \& Whitin (1958) as mentioned in section 4.2.1.

\subsubsection{Zero-Inventory-Ordering property}

In a ZIO-type planning, production would only be planned when there is no product remaining in inventory. We demonstrate that an optimal solution can always be found within all possible plans that respects the ZIO property. Moreover, since only ZIO type plans need to be investigated in finding the optimal solution, the number of feasible solutions to investigate is significantly reduced. The ZIO property can be expressed as follows:

Theorem 4.5.1. There exists an optimal production plan in which $I_{t-1} \times Q_{t}=0$ for all $t$ (where $I_{t}$ is the inventory at the end of the period $t)$ in $U L S_{P(B F R)}$ problem.

The proof is presented in the below sub-section. The main idea is to demonstrate that we can always improve the objective value by eliminating violation(s) of the property by transforming a production plan which does not comply with the ZIO property to a ZIO-type plan. Eventually, we can prove the ZIO-type production program provides the optimal objective value.

\subsubsection{Proof of Theorem}

Firstly, we denote that:

- $X^{U L S_{P(W C R)}}$ represents all feasible solution in term of $X_{t k}$;

- $P$ is a production plan;

- Profit $(P)$ stands for the objective value in the proposed model with $P$.

Definition 1. A production planning is complying with the ZIO property if and only if the production is only planned when the inventory level drops to zero. With our notation, it can mathematically can be expressed as

$$
Q_{t} \times\left[\sum_{k=1}^{t-1}\left(\sum_{l=t}^{T} X_{k, l}\right)\right]=0 \quad \forall t \in[2, T]
$$

In other words, it can be written as :

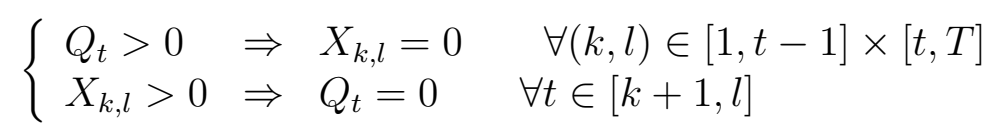

Conjecture 4.5.2. There is a plan $P^{*} \in X^{\left(U L S_{P(B F R)}\right)}$ which verifies the ZIO property while

$$
\operatorname{Profit}\left(P^{*}\right)=\max _{P \in X^{\left(U L S_{P(B F R)}\right)}} \operatorname{Profit}(P)
$$

Let $(P) \in X^{\left(U L S_{P(B F R)}\right)}$ be a planning which does not complies with the ZIO property. Note that $(t, m, n) \in[1, T]^{3}$ is a triplet with $m<t \leq n$ and a violation of the property can be presented as $Q_{t} \times X_{m, n}>0$. Then, we assume that several violations can be found in $P$. 
We consider a plan $P^{\prime}$ as a plan derived by $P$ in the following way :

$$
\begin{array}{ll}
X_{k, t}^{\prime}=X_{k, t} & \forall(k, t) \in[1, T]^{2}-\{(m, n),(t, n)\} \\
X_{m, n}^{\prime}=0 & \\
X_{t, n}^{\prime}=X_{t, n}+X_{m, n} &
\end{array}
$$

According to up-mentioned definition, we find one less violation of the property in $P^{\prime}$ than in $P$. Now, we need to prove that $\operatorname{Profit}(P)-\operatorname{Profit}\left(P^{\prime}\right) \leq 0$. To do so, we go through each term of the objective function. To simplify the NPV formulation, note that $a_{t}^{\prime}=a \frac{1}{(1+\alpha)^{t}}, s_{t}^{\prime}=s_{\frac{1}{(1+\alpha)^{t}}}$, $p_{t}^{\prime}=p \frac{1}{(1+\alpha)^{t}}, h_{t}^{\prime}=h \frac{1}{(1+\alpha)^{t}}$ and $v_{t}^{\prime}=v \frac{1}{(1+\alpha)^{t}}$. Essentially, these cost parameters are decreasing over time horizon.

In the following proof, we examine the difference of all terms in objective function with $P$ and $P^{\prime}$, respectively the revenue, the logistic cost and the OWCR financing cost. At first, there is no difference in NPV of Revenue between these two plans. It is because the demand and unit selling price (as well as discounted unit selling price.) are identical over horizon. Thus, $R(P)-R\left(P^{\prime}\right)=0$. Then, the difference in logistic cost can be written as :

$$
\begin{aligned}
L C(P)-L C\left(P^{\prime}\right) & =\sum_{j=1}^{T}\left[a_{j+r_{f}}^{\prime}\left(Q_{j}-Q_{j}^{\prime}\right)+p_{j}^{\prime}\left(Q_{j}-Q_{j}^{\prime}\right)+s_{j}^{\prime}\left(Y_{j}-Y_{j}^{\prime}\right)+h_{j}^{\prime}\left(I_{j}-I_{j}^{\prime}\right)\right] \\
& =X_{m, n}\left(a_{m+r_{f}}^{\prime}-a_{t+r_{f}}^{\prime}\right)+X_{m, n}\left(p_{m}^{\prime}-p_{t}^{\prime}\right)+s_{t}^{\prime}\left(Y_{m}-Y_{m}^{\prime}\right)+X_{m, n} \sum_{j=m}^{t-1} h_{j}^{\prime}
\end{aligned}
$$

Since $m<t$ and $Y_{m} \geq Y_{m}^{\prime}$, we obtain that :

$$
L C(P)-L C\left(P^{\prime}\right)>0
$$

Next, for OWCR financing cost, we examine its components one by one :

Firstly, since we apply constant unit costs in calculation of purchasing, production and inventory holding costs, comparison on these operations are relatively direct as follows :

$$
\begin{aligned}
& W C R^{\text {pur }}(P)-W C R^{\text {pur }}\left(P^{\prime}\right)=\sum_{t=1}^{T} \sum_{k=t}^{T} a X_{t k}\left[\sum_{j=t}^{T} \frac{1}{(1+\alpha)^{j+r_{f}}}-\sum_{j=k}^{T} \frac{1}{(1+\alpha)^{j+r_{c}}}\right] \\
& -\sum_{t=1}^{T} \sum_{k=t}^{T} a X_{t k}^{\prime}\left[\sum_{j=t}^{T} \frac{1}{(1+\alpha)^{j+r_{f}}}-\sum_{j=k}^{T} \frac{1}{(1+\alpha)^{j+r_{c}}}\right] \\
& =X_{m, n}\left[\sum_{j=m}^{T} a_{j}^{\prime}-\sum_{j=t}^{T} a_{j}^{\prime}\right]=X_{m, n} \sum_{j=m}^{t-1} a_{j}^{\prime}>0 \\
& W C R^{\text {prod }}(P)-W C R^{\text {prod }}\left(P^{\prime}\right)=\sum_{t=1}^{T} \sum_{k=t}^{T} p X_{t k} \sum_{j=t}^{k+r_{c}-1} \frac{1}{(1+\alpha)^{t}}-\sum_{t=1}^{T} \sum_{k=t}^{T} p X_{t k}^{\prime} \sum_{j=t}^{k+r_{c}-1} \frac{1}{(1+\alpha)^{t}} \\
& =X_{m, n}\left[\sum_{j=m}^{k+r_{c}-1} p_{j}^{\prime}-\sum_{j=t}^{k+r_{c}-1} p_{j}^{\prime}\right]=X_{m, n} \sum_{j=m}^{t-1} p_{j}^{\prime}>0 \\
& W C R^{i n v}(P)-W C R^{i n v}\left(P^{\prime}\right)=\sum_{t=1}^{T} \sum_{k=t}^{T} h X_{t k} \sum_{l=t}^{k-1} \sum_{j=l}^{k+r_{c}-1} \frac{1}{(1+\alpha)^{t}}-\sum_{t=1}^{T} \sum_{k=t}^{T} h X_{t k}^{\prime} \sum_{l=t}^{k-1} \sum_{j=l}^{k+r_{c}-1} \frac{1}{(1+\alpha)^{t}} \\
& =X_{m, n}\left(\sum_{l=m}^{n-1} \sum_{j=l}^{n+r_{c}-1} h_{j}^{\prime}-\sum_{l=t}^{n-1} \sum_{j=l}^{n+r_{c}-1} h_{j}^{\prime}\right) \\
& =X_{m, n}\left(\sum_{l=m}^{m+n-t-1} \sum_{k=m}^{t-1}\left(h_{k}^{\prime} \frac{1}{(1+\alpha)^{l}}\right)+\sum_{l=m+n-t}^{n-1} \sum_{j=l}^{n+r_{c}-1} h_{j}^{\prime}\right)>0
\end{aligned}
$$


Since the cost parameters are all positive, we confirm that the OWCR of these three operations is greater with $P$ than the OWCR with $P^{\prime}$.

At last, we focus on the difference in OWCR of set-up. The difficulty comes from the fact that the shared set-up cost per product (in period $m$ and $t$ ) changes with the quantity of $X_{t k}$ :

$$
\begin{aligned}
& W C R^{s e t}(P)-W C R^{s e t}\left(P^{\prime}\right)=\sum_{t=1}^{T} \sum_{k=t}^{T} \frac{s Y_{t} X_{t k}}{Q_{t}+\left(1-Y_{t}\right)} X_{t k} \sum_{j=t}^{k+r_{c}-1} \frac{1}{(1+\alpha)^{t}} \\
& -\sum_{t=1}^{T} \sum_{k=t}^{T} \frac{s Y_{t} X_{t k}}{Q_{t}+\left(1-Y_{t}\right)} X_{t k} \sum_{j=t}^{k+r_{c}-1} \frac{1}{(1+\alpha)^{t}} \\
& =\sum_{k=m}^{T} X_{m, k} \sum_{j=m}^{k+r_{c}-1} \frac{s_{j}^{\prime} Y_{m}}{Q_{m}+\left(1-Y_{m}\right)}+\sum_{k=t}^{T} X_{t k} \sum_{j=t}^{k+r_{c}-1} \frac{s_{j}^{\prime} Y_{t}}{Q_{t}+\left(1-Y_{t}\right)} \\
& -\sum_{k=m}^{T} X_{m, k}^{\prime} \sum_{j=m}^{k+r_{c}-1} \frac{s_{j}^{\prime} Y_{m}^{\prime}}{Q_{m}^{\prime}+\left(1-Y_{m}^{\prime}\right)}-\sum_{k=t}^{T} X_{t k}^{\prime} \sum_{j=t}^{k+r_{c}-1} \frac{s_{j}^{\prime} Y_{t}^{\prime}}{Q_{t}^{\prime}+\left(1-Y_{t}^{\prime}\right)} \\
& =\underbrace{\sum_{k=m}^{T}\left(X_{m, k} \sum_{j=m}^{k+r_{c}-1} \frac{s_{j}^{\prime} Y_{m}}{Q_{m}+\left(1-Y_{m}\right)}-X_{m, k}^{\prime} \sum_{j=m}^{k+r_{c}-1} \frac{s_{j}^{\prime} Y_{m}^{\prime}}{Q_{m}^{\prime}+\left(1-Y_{m}^{\prime}\right)}\right)}_{\text {Part } 1} \\
& +\underbrace{\sum_{k=t}^{T}\left(X_{t k} \sum_{j=t}^{k+r_{c}-1} \frac{s_{j}^{\prime} Y_{t}}{Q_{t}+\left(1-Y_{t}\right)}-X_{t k}^{\prime} \sum_{j=t}^{k+r_{c}-1} \frac{s_{j}^{\prime} Y_{t}^{\prime}}{Q_{t}^{\prime}+\left(1-Y_{t}^{\prime}\right)}\right)}_{\text {Part } 2}
\end{aligned}
$$

Next, we evaluate the part 1. Two possible cases are discussed in the following paragraphs :

Case 1: $Q_{m}^{\prime}=0$. In this case,

$$
\text { Part } 1=\sum_{k=m}^{T} X_{m, k} \sum_{j=m}^{k+r_{c}-1} \frac{s_{j}^{\prime} Y_{m}}{Q_{m}+\left(1-Y_{m}\right)}=\sum_{k=m}^{T} X_{m, k} \sum_{j=m}^{k+r_{c}-1} \frac{s_{j}^{\prime}}{Q_{m}}>0
$$

Case 2: $Q_{m}^{\prime}>0$. In this case,

$$
\begin{aligned}
\text { Part 1 } & =\sum_{k=m}^{T}\left(X_{m, k} \sum_{j=m}^{k+r_{c}-1} \frac{s_{j}^{\prime} Y_{m}}{Q_{m}+\left(1-Y_{m}\right)}-X_{m, k}^{\prime} \sum_{j=m}^{k+r_{c}-1} \frac{s_{j}^{\prime} Y_{m}^{\prime}}{Q_{m}^{\prime}+\left(1-Y_{m}^{\prime}\right)}\right) \\
& =\sum_{k=m}^{T}\left(X_{m, k} \sum_{j=m}^{k+r_{c}-1} \frac{s_{j}^{\prime}}{Q_{m}}-X_{m, k}^{\prime} \sum_{j=m}^{k+r_{c}-1} \frac{s_{j}^{\prime}}{Q_{m}^{\prime}}\right) \\
& =\frac{1}{Q_{m} Q_{m}^{\prime}} \sum_{k=m}^{T} \sum_{j=m}^{k+r_{c}-1} s_{j}^{\prime}\left(X_{m, k} Q_{m}^{\prime}-X_{m, k}^{\prime} Q_{m}\right) \\
& =\frac{1}{Q_{m} Q_{m}^{\prime}}\left[\sum_{j=m}^{n+r_{c}-1} s_{j}^{\prime} X_{m, n} Q_{m}^{\prime}+\sum_{k=m, k \neq n}^{T} \sum_{j=m}^{k+r_{c}-1} s_{j}^{\prime}\left(X_{m, k}^{\prime} Q_{m}^{\prime}-X_{m, k}^{\prime} Q_{m}\right)\right]
\end{aligned}
$$




$$
\begin{aligned}
& =\frac{1}{Q_{m} Q_{m}^{\prime}}\left[\sum_{j=m}^{n+r_{c}-1} s_{j}^{\prime} X_{m, n} Q_{m}^{\prime}+\sum_{k=m, k \neq n}^{T} \sum_{j=m}^{k+r_{c}-1} s_{j}^{\prime}\left(X_{m, k}^{\prime} Q_{m}^{\prime}-X_{m, k}^{\prime}\left(Q_{m}^{\prime}+X_{m, n}\right)\right)\right] \\
& =\frac{1}{Q_{m} Q_{m}^{\prime}}\left[\sum_{j=m}^{n+r_{c}-1} s_{j}^{\prime} X_{m, n} Q_{m}^{\prime}-\sum_{k=m, k \neq n}^{T} \sum_{j=m}^{k+r_{c}-1} s_{j}^{\prime} X_{m, k}^{\prime} X_{m, n}\right] \\
& =\frac{X_{m, n}}{Q_{m} Q_{m}^{\prime}}\left[\sum_{j=m}^{n+r_{c}-1} s_{j}^{\prime} Q_{m}^{\prime}-\sum_{k=m, k \neq n}^{T} \sum_{j=m}^{k+r_{c}-1} s_{j}^{\prime} X_{m, k}^{\prime}\right] \\
& =\frac{X_{m, n}}{Q_{m} Q_{m}^{\prime}}\left[\sum_{j=m}^{n+r_{c}-1} s_{j}^{\prime} \sum_{k=m}^{t-1} X_{m, k}^{\prime}-\sum_{k=m}^{t-1} \sum_{j=m}^{k+r_{c}-1} s_{j}^{\prime} X_{m, k}^{\prime}\right] \\
& =\frac{X_{m, n}}{Q_{m} Q_{m}^{\prime}} \sum_{k=m}^{t-1} X_{m, k}\left(\sum_{j=m}^{n+r_{c}-1} s_{j}^{\prime}-\sum_{j=m}^{k+r_{c}-1} s_{j}^{\prime}\right) \\
& =\frac{X_{m, n}}{Q_{m} Q_{m}^{\prime}} \sum_{k=m}^{t-1} X_{m, k} \sum_{j=k+r_{c}-1}^{n+r_{c}-1} s_{j}^{\prime}>0
\end{aligned}
$$

Thus, the part 1 is always positive in all cases. Then, we go through the part 2 :

$$
\begin{aligned}
\text { Part } 2 & =\sum_{k=t}^{T}\left(X_{t k} \sum_{j=t}^{k+r_{c}-1} \frac{s_{j}^{\prime} Y_{t}}{Q_{t}+\left(1-Y_{t}\right)}-X_{t k}^{\prime} \sum_{j=t}^{k+r_{c}-1} \frac{s_{j}^{\prime} Y_{t}^{\prime}}{Q_{t}^{\prime}+\left(1-Y_{t}^{\prime}\right)}\right) \\
& =\sum_{k=t}^{T}\left(\frac{X_{t k}}{Q_{t}} \sum_{j=t}^{k+r_{c}-1} s_{j}^{\prime}-\frac{X_{t k}^{\prime}}{Q_{t}^{\prime}} \sum_{j=t}^{k+r_{c}-1} s_{j}^{\prime}\right) \\
& =\frac{1}{Q_{t} Q_{t}^{\prime}} \sum_{k=t}^{T}\left(X_{t k} Q_{t}^{\prime} \sum_{j=t}^{k+r_{c}-1} s_{j}^{\prime}-X_{t k}^{\prime} Q_{t} \sum_{j=t}^{k+r_{c}-1} s_{j}^{\prime}\right) \\
& =\frac{1}{Q_{t} Q_{t}^{\prime}} \sum_{k=t}^{T}\left(X_{t k}\left(Q_{t}+X_{m, n}\right) \sum_{j=t}^{k+r_{c}-1} s_{j}^{\prime}-X_{t k}^{\prime} Q_{t} \sum_{j=t}^{k+r_{c}-1} s_{j}^{\prime}\right) \\
& =\frac{1}{Q_{t} Q_{t}^{\prime}} \sum_{k=t}^{T}\left(X_{t k} X_{m, n} \sum_{j=t}^{k+r_{c}-1} s_{j}^{\prime}-Q_{t}\left(X_{t k}-X_{t k}^{\prime}\right) \sum_{j=t}^{k+r_{c}-1} s_{j}^{\prime}\right) \\
& =\frac{1}{Q_{t} Q_{t}^{\prime}}\left(\sum_{k=t}^{T} X_{t k} X_{m, n} \sum_{j=t}^{k+r_{c}-1} s_{j}^{\prime}-Q_{t} X_{m, n} \sum_{j=t}^{n+r_{c}-1} s_{j}^{\prime}\right)
\end{aligned}
$$

The element which clearly causes a problem in the different cases is $\frac{X_{m, n}}{Q_{t}^{\prime}} \sum_{j=t}^{n+r_{c}-1} s_{j}^{\prime}$. Two possibilities are to examine:

Case A : $Q_{t}^{\prime} \geq Q_{m}$. We have

$$
\frac{X_{m, n}}{Q_{t}^{\prime}} \sum_{j=n}^{n+r_{c}-1} s_{j}^{\prime}<\frac{X_{m, n}}{Q_{m}} \sum_{j=n}^{n+r_{c}-1} s_{j}^{\prime}
$$

Then, we attempt to compare this element with the part 1 : 
Case A-1: $Q_{m}^{\prime}=0$. In this case,

$$
\begin{aligned}
\text { Part 1 } & =\sum_{k=m}^{T} \frac{X_{m, k}}{Q_{m}} \sum_{j=m}^{k+r_{c}-1} s_{j}^{\prime} \\
& =\sum_{k=m, k \neq n}^{T} \frac{X_{m, k}}{Q_{m}} \sum_{j=m}^{k+r_{c}-1} s_{j}^{\prime}+\frac{X_{m, n}}{Q_{m}} \sum_{j=n}^{n+r_{c}-1} s_{j}^{\prime} \\
& \geq \frac{X_{m, n}}{Q_{m}} \sum_{j=n}^{n+r_{c}-1} s_{j}^{\prime} q \\
& \geq \frac{X_{m, n}}{Q_{m}} \sum_{j=n}^{n+r_{c}-1} s_{j}^{\prime}
\end{aligned}
$$

Then, Part $1+$ Part $2 \geq \frac{X_{m, n}}{Q_{m}} \sum_{j=n}^{n+r_{c}-1} s_{j}^{\prime}-\frac{X_{m, n}}{Q_{t}^{\prime}} \sum_{j=n}^{n+r_{c}-1} s_{j}^{\prime}$

$$
\geq\left(\frac{X_{m, n}}{Q_{t}^{\prime}}-\frac{X_{m, n}}{Q_{t}^{\prime}}\right) \sum_{j=n}^{n+r_{c}-1} s_{j}^{\prime}>0 \text { with } Q_{t}^{\prime} \geq Q_{m}
$$

Case A-2: $Q_{m}^{\prime}>0$. In this case,

$$
\begin{aligned}
\text { Part 1 } & =\frac{X_{m, n}}{Q_{m} Q_{m}^{\prime}} \sum_{k=m}^{t-1} X_{m, k} \sum_{j=k+r_{c}-1}^{n+r_{c}-1} s_{j}^{\prime} \\
& =\frac{X_{m, n}}{Q_{m} Q_{m}^{\prime}} \sum_{k=m}^{t-1} X_{m, k}\left(\sum_{j=m}^{n+r_{c}-1} s_{j}^{\prime}-\sum_{j=m}^{k+r_{c}-1} s_{j}^{\prime}\right) \\
\geq & \frac{X_{m, n}}{Q_{m} Q_{m}^{\prime}} \sum_{k=m}^{t-1} X_{m, k}\left(\sum_{j=m}^{n+r_{c}-1} s_{j}^{\prime}-\sum_{j=m}^{t+r_{c}-1} s_{j}^{\prime}\right) \\
& \geq \frac{X_{m, n}}{Q_{m}}\left(\sum_{j=m}^{n+r_{c}-1} s_{j}^{\prime}-\sum_{j=m}^{t+r_{c}-1} s_{j}^{\prime}\right) \\
& \geq \frac{X_{m, n}}{Q_{t}^{\prime}}\left(\sum_{j=m}^{n+r_{c}-1} s_{j}^{\prime}-\sum_{j=m}^{t+r_{c}-1} s_{j}^{\prime}\right) \\
& =\frac{X_{m, n}}{Q_{t}^{\prime}} \sum_{j=t+r_{c}-1}^{n+r_{c}-1} s_{j}^{\prime}
\end{aligned}
$$

Moreover, we have :

$$
\begin{gathered}
\frac{1}{Q_{t} Q_{t}^{\prime}} \sum_{k=t}^{T} X_{t k} X_{m, n} \sum_{j=t}^{k+r_{c}-1} s_{j}^{\prime} \geq \\
\frac{X_{m, n}}{Q_{t} Q_{t}^{\prime}} \sum_{k=t}^{T} X_{t k} \sum_{j=t}^{k+r_{c}-1} s_{j}^{\prime} \geq \\
\frac{X_{m, n}}{Q_{t} Q_{t}^{\prime}} \sum_{k=t}^{T} X_{t k} \sum_{j=t}^{t+r_{c}-1} s_{j}^{\prime} \geq \\
\frac{X_{m, n}}{Q_{t} Q_{t}^{\prime}} \sum_{j=t}^{t+r_{c}-1} s_{j}^{\prime} \sum_{k=t}^{T} X_{t k} \geq \\
\frac{X_{m, n}}{Q_{t}^{\prime}} \sum_{j=t}^{t+r_{c}-1} s_{j}^{\prime}
\end{gathered}
$$


Thus, with 4.37) and 4.38,

$$
\text { Part } 1+\text { Part2 } \geq \frac{X_{m, n}}{Q_{t}^{\prime}} \sum_{j=t+r_{c}-1}^{n+r_{c}-1} s_{j}^{\prime}+\frac{X_{m, n}}{Q_{t}^{\prime}} \sum_{j=t}^{t+r_{c}-1} s_{j}^{\prime}-\frac{X_{m, n}}{Q_{t}^{\prime}} \sum_{j=t}^{n+r_{c}-1} s_{j}^{\prime} \geq 0
$$

In this case, the sum of Part 1 and Part 2 is also positive.

\section{Case B : $Q_{t}^{\prime}<Q_{m}$.}

We distinguish again both cases of part 1:

Case B-1: $Q_{m}^{\prime}=0$. Impossible because $Q_{t}^{\prime} \geq Q_{m}$.

Case B-2: $Q_{m}^{\prime}>0$. The part 1 can be transformed as follows :

$$
\begin{aligned}
\frac{X_{m, n}}{Q_{m} Q_{m}^{\prime}} \sum_{k=m}^{t-1} X_{m, k} \sum_{j=k+r_{c}-1}^{n+r_{c}-1} s_{j}^{\prime} & \geq \\
\frac{X_{m, n}}{Q_{m} Q_{m}^{\prime}} \sum_{j=t+r_{c}-2}^{n+r_{c}-1} s_{j}^{\prime} \sum_{k=m}^{t-1} X_{m, k} & \geq \\
\frac{X_{m, n}}{Q_{m} Q_{m}^{\prime}} \sum_{j=t+r_{c}-2}^{n+r_{c}-1} s_{j}^{\prime} Q_{m}^{\prime} & \geq \\
\frac{X_{m, n}}{Q_{m}} \sum_{j=t+r_{c}-2}^{n+r_{c}-1} s_{j}^{\prime} &
\end{aligned}
$$

The part 2 can be formulated as :

$$
\begin{aligned}
& \frac{1}{Q_{t} Q_{t}^{\prime}}\left(\sum_{k=t}^{T} X_{t k} X_{m, n} \sum_{j=t}^{k+r_{c}-1} s_{j}^{\prime}-Q_{t} X_{m, n} \sum_{j=t}^{n+r_{c}-1} s_{j}^{\prime}\right)= \\
& \frac{X_{m, n}}{Q_{t} Q_{t}^{\prime}}\left(\sum_{k=t}^{T} X_{t k} \sum_{j=t}^{k+r_{c}-1} s_{j}^{\prime}-Q_{t} \sum_{j=t}^{n+r_{c}-1} s_{j}^{\prime}\right) \geq \\
& \frac{X_{m, n}}{Q_{t} Q_{t}^{\prime}}\left(\sum_{j=t}^{t+r_{c}-1} s_{j}^{\prime}\left(\sum_{k=t}^{T} X_{t k}\right)-Q_{t} \sum_{j=t}^{n+r_{c}-1} s_{j}^{\prime}\right) \geq \\
& \frac{X_{m, n}}{Q_{t} Q_{t}^{\prime}}\left(\sum_{j=t}^{t+r_{c}-1} s_{j}^{\prime} Q_{t}-Q_{t} \sum_{j=t}^{n+r_{c}-1} s_{j}^{\prime}\right) \geq \\
& -\frac{X_{m, n}}{Q_{t}^{\prime}} \sum_{j=t+r_{c}-1}^{n+r_{c}-1} s_{j}^{\prime} \geq \\
& -\frac{X_{m, n}}{Q_{m}} \sum_{j=t+r_{c}-1}^{n+r_{c}-1} s_{j}^{\prime}
\end{aligned}
$$

In consequence,

$$
\text { Part } 1+\text { Part } 2=\frac{X_{m, n}}{Q_{m}} \sum_{j=t+r_{c}-2}^{n+r_{c}-1} s_{j}^{\prime}-\frac{X_{m, n}}{Q_{m}} \sum_{j=t+r_{c}-1}^{n+r_{c}-1} s_{j}^{\prime} \geq \frac{X_{m, n}}{Q_{m}} s_{t+r_{c}-2}^{\prime} \geq 0
$$

To summarize, the sum of part 1 and part 2 is always positive in all cases which means $P$ generates a bigger OWCR financing cost than $P^{\prime}$. In summary of all elements, the NPV of revenue is equal with these two plans, but the NPV of the total cost is greater with $P$. Therefore, we favorite the $P^{\prime}$ which have one less violation of ZIO property. In general, we are able to deduce that the optimal planning is the ZIO type planning with no violation at all. 


\subsubsection{Algorithm description}

Since the demand, the delay in payment from the customers, and the discounted rate are all given, the NPV of revenue is fixed for each instance. Thus, we only need to minimize the NPV of all costs. Then, like the classical shortest path problem, the $U L S_{P(B F R)}$ problem is formulated with an acyclic oriented graph $G=\{V, E\}$ (see Figure 4.2). The nodes $V_{t}$ represent the $T$ periods in the planning horizon including a dummy node at the end (i.e., node 6 in Figure 4.2). Moreover, determining the production quantity benefits from the ZIO property which means that the production quantity can only be the sum of demands in the following periods. Therefore, we avoid the difficulty caused by the nonlinear formulation of setup cost to finance. Consequently, an arc $E_{t k}$ denotes a production planned in period $t$ for all demands between periods $t$ and $k-1$. With this concept and the ZIO property, we determine the precise quantity and period of a production as well as the delivery periods. We are thus able to deduce the period of client's payment for financial cost calculation. Therefore, finding a shortest path allows us to obtain the optimal production program with the minimum total NPV of all costs generated. Furthermore, with the dummy node, we are able to express all types of production lots in this acyclic graph including the "make-to-order" type production.

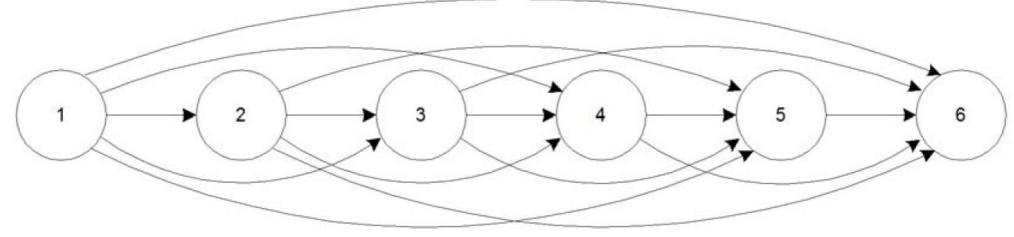

Figure 4.2 - Reformulation in shortest path problem

In the objective function of $U L S_{P(W C R)}$, an aggregate OWCR formulation is given. However, a $X_{t k}$-based formulation of NPV of OWCR is required for the arc value calculations. In the former formulation, the OWCR of an operation is the product of the corresponding unit cost, $X_{t k}$ and the number of periods to finance. Considering the NPV of OWCR, the discount rates are different (decreasing) during the periods to finance. Thereby, the discounted OWCR depends on the period where the payment occurred. $W C R_{t k}^{p u r}, W C R_{t k}^{s e t}, W C R_{t k}^{p r o d}$ and $W C R_{t k}^{I n v}$ respectively stand for the OWCR generated by operations of purchasing, set-up, production and inventory holding for producing the $X_{t k}$. For example, in purchasing raw material for the production of $X_{t k}$, the payment to supplier is executed in period $t+r_{f}$ and the client's payment is received in period $k+r_{c}$. Therefore, the corresponding discounted OWCR is all financial needs to financed between $t+r_{f}$ and $k+r_{c}$. However, we cannot determinate whether the payment to supplier or the client's payment would occur first. For this reason, we consider that we continue to finance the payment to the supplier until the end of the planning horizon as well as the payment from customer. The mathematical formulation can be written as $W C R_{t k}^{p u r}=a X_{t k}\left[\sum_{j=t+r_{f}}^{T} \frac{1}{(1+\alpha)^{j}}-\sum_{j=k+r_{c}}^{T} \frac{1}{(1+\alpha)^{j}}\right]$. (By considering the financing cost of OWCR, the horizon is extended because some payments may be received from client or be sent to supplier after the last period of the planning horizon.)

This formula allows us to correctly cover the two cases whether the payment to supplier or client's payment occurs first. In the first case, a negative OWCR in purchasing will be obtained which can be considered as an additional refund for the OWCR of other operations. In the second case, $W C R_{t k}^{p u r}$ is a positive financial need to finance.

Following the same concept, the OWCR in other operations can be formulated as

$$
\begin{aligned}
& -W C R_{t k}^{s e t}=\frac{s Y_{t}}{Q_{t}+\left(1-Y_{t}\right)} X_{t k} \sum_{j=t}^{k+r_{c}-1} \frac{1}{(1+\alpha)^{j}}, \\
& -W C R_{t k}^{p r o d}=p X_{t k} \sum_{j=t}^{k+r_{c}-1} \frac{1}{(1+\alpha)^{j}},
\end{aligned}
$$


— and $W C R_{t k}^{I n v}=h X_{t k} \sum_{l=t}^{k-1} \sum_{j=l}^{k+r_{c}-1} \frac{1}{(1+\alpha)^{j}}$.

For WCR of inventory holding, if $k=t$ (i.e., $X_{t k}$ is produced for demand in the same period), then there is no inventory holding cost. Combining all these components, the total OWCR for all $X_{t k}$, denoted as $W C R_{\text {total }}$, can be written as follows :

$$
W C R_{t o t a l}=\sum_{t=1}^{T} \sum_{k=1}^{T}\left(W C R_{t k}^{p u r}+W C R_{t k}^{s e t}+W C R_{t k}^{\text {prod }}+W C R_{t k}^{I n v}\right)
$$

To simplify the NPV formulation, note that $a_{t}^{\prime}=a_{\frac{1}{(1+\alpha)^{t}}}, s_{t}^{\prime}=s \frac{1}{(1+\alpha)^{t}}, p_{t}^{\prime}=p \frac{1}{(1+\alpha)^{t}}$ and $h_{t}^{\prime}=$ $h \frac{1}{(1+\alpha)^{t}}$. With the ZIO property, the arc values are presented as follows (with $L C_{t k}$ representing all the production-related costs):

$$
\begin{aligned}
E_{t k}= & L C_{t k}+\beta \sum_{l=t}^{k-1}\left(W C R_{t k}^{\text {pur }}+W C R_{t k}^{s e t}+W C R_{t k}^{\text {prod }}+W C R_{t k}^{I n v}\right) \\
= & \left(a_{t+r_{f}}^{\prime}+p_{t}^{\prime}\right) \sum_{l=t}^{k-1} d_{l}+s_{t}^{\prime}+\sum_{l=t}^{k-1} d_{l} \sum_{i=t}^{l-1} h_{i}^{\prime} \\
& +\beta \sum_{l=t}^{k-1} d_{l}\left[\left(\sum_{j=t}^{T} a_{j+r_{f}}^{\prime}-\sum_{j=l}^{T} a_{j+r_{c}}^{\prime}\right)+\sum_{j=t}^{l+r_{c}-1} p_{j}^{\prime}+\sum_{j=t}^{l+r_{c}-1} \frac{s_{j}^{\prime}}{\sum_{i=t}^{k-1} d_{i}}+\sum_{i=t}^{l-1} \sum_{j=i}^{l+r_{c}-1} h_{j}^{\prime}\right]
\end{aligned}
$$

The computation of $E_{t k}$ is done in $\mathrm{O}\left(T^{2}\right)$. The objective of this algorithm is to find the shortest path to the last node. To do so, we need to progressively locate the shortest path to other nodes. Thereby, we examine all possible paths to each node from all its predecessors through the arc in between. This can be realized by the recursive function presented as follows :

$$
\operatorname{Cost}[t]=\min _{j \in[1, t-1]}\left\{\operatorname{Cost}[j]+E_{j t}\right\}
$$

with $\operatorname{Cost}[t]$ the minimal total cost to finance to satisfy all demands until period $t$. Therefore, a dynamic programming algorithm is established as presented in Algorithm 1. The complexity of the algorithm is $\mathrm{O}\left(T^{4}\right)$, thus the $U L S_{P(W C R)}$ problem can be solved in polynomial time.

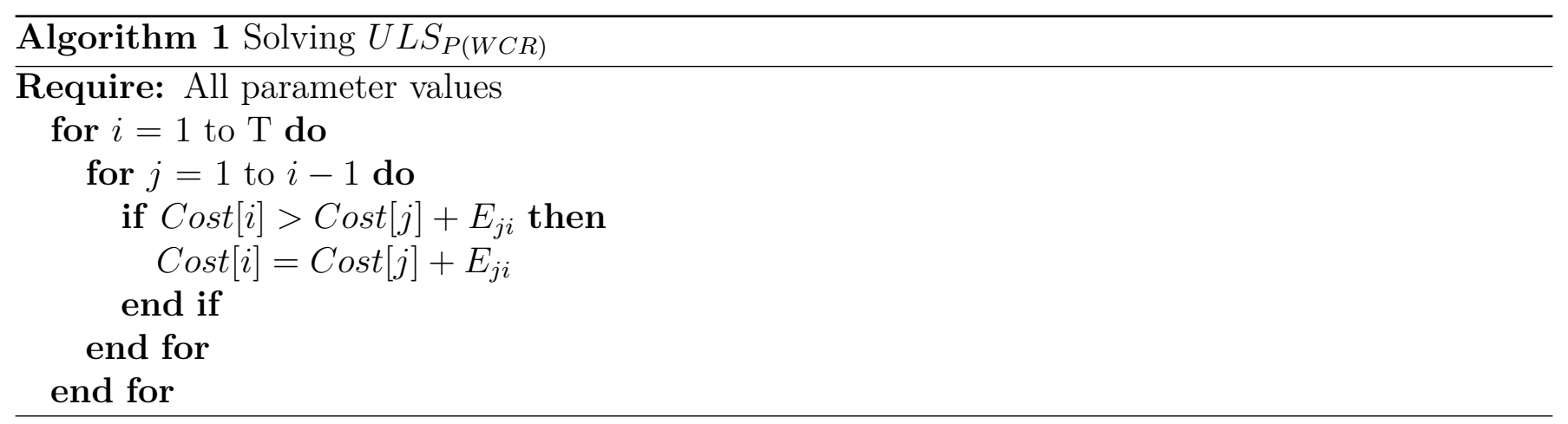

\subsection{Numerical analysis}

Through the following numerical tests, we reveal differences between optimal production plans for profit maximization or costs minimization objectives. These tests illustrate the influence of considering the financing cost of OWCR on an optimal production program. With the proven ZIO 
property, the optimal program of profit maximization problem is calculated and denoted as $P_{w c r}^{o p t}$. Moreover, the optimal program of the traditional $U L S$ model is denoted as $P_{u l s}^{o p t}$. The tests are organized as follows:

- Firstly, a comparison between $P_{w c r}^{o p t}$ and $P_{u l s}^{o p t}$ with a same set of parameter values is provided to show the differences in the production programs;

- Secondly, the evolution of a production program following the variation of discount and interest rates is presented as well as the change of average inventory level;

- Thirdly, we compare the objective value of $U L S$ and $U L S_{P(W C R)}$ models by applying respectively $P_{u l s}^{o p t}$ and $P_{w c r}^{o p t}$ in both models.

For the following tests, we adopt the demand of the instance of Trigeiro et al. (1989) (G-72, demand 7). Values of other parameters are set to adapt the ULS concept which considers only setup and inventory holding costs (see Table 4.8):

- Since the purchasing and production cost are not considered in traditional ULS model, these unit costs are given as zero in order to compare the optimal programs in the same conditions. Accordingly, the delay in payment to the supplier involved only in purchasing is irrelevant in determining the production planning. Thus, $a=p=r_{f}=0$;

- The setup cost, $s$, is given as 600 and the unit cost of inventory holding per period, $h$, is fixed to 1 ;

- The delay in payments from customer, $r_{c}$ is set to 5 periods;

- The unit selling price will not be considered as it is a constant parameter and will not impact the production program. However, it should be taken into account for the periodic OWCR maximization objective.

Table 4.8 - Numerical example parameter values

\begin{tabular}{ll}
\hline Parameter & Value \\
\hline Purchasing unit cost, $a$ & 0 \\
Setup cost, $s$ & 600 \\
Production unit cost, $p$ & 0 \\
Inventory holding unit cost per period, $h$ & 1 \\
Delay in payment from customer, $r_{c}$ & 5 \\
Delay in payment to supplier, $r_{f}$ & 0 \\
Unit sales price & not considered \\
\hline
\end{tabular}

\subsubsection{Production program comparisons}

We compare optimal programs separately calculated by these two models with $\alpha=0.08$ and $\beta=0.1 . P_{w c r}^{o p t}$ and $P_{u l s}^{o p t}$ are illustrated in figure 4.3 . We observe that

- The production programs are different. The $U L S$ model can not always provide the optimal solution for maximizing the profit;

- The production lots of $P_{w c r}^{o p t}$ are smaller with a profit maximization objective compared to the ones in $P_{u l s}^{o p t}$. More setups are planned in $P_{w c r}^{o p t}$ that results in a lower average inventory levels;

This difference in production plans is mainly caused by the cumulative effect of financing the inventory holding costs. In $U L S_{P(W C R)}$ model, all purchasing, setup, production and inventory holding costs have a financial consequence by considering the financial cost to cover them before receiving customer's payment. As shown in Table 4.7, the duration to be financed is much more greater for inventory holding cost compared to the one for setup cost. It means that the financial cost of inventory holding cost varies more rapidly than the one of setup cost. Therefore, when we are looking for a trade-off between the setup related and inventory holding related costs, we would 


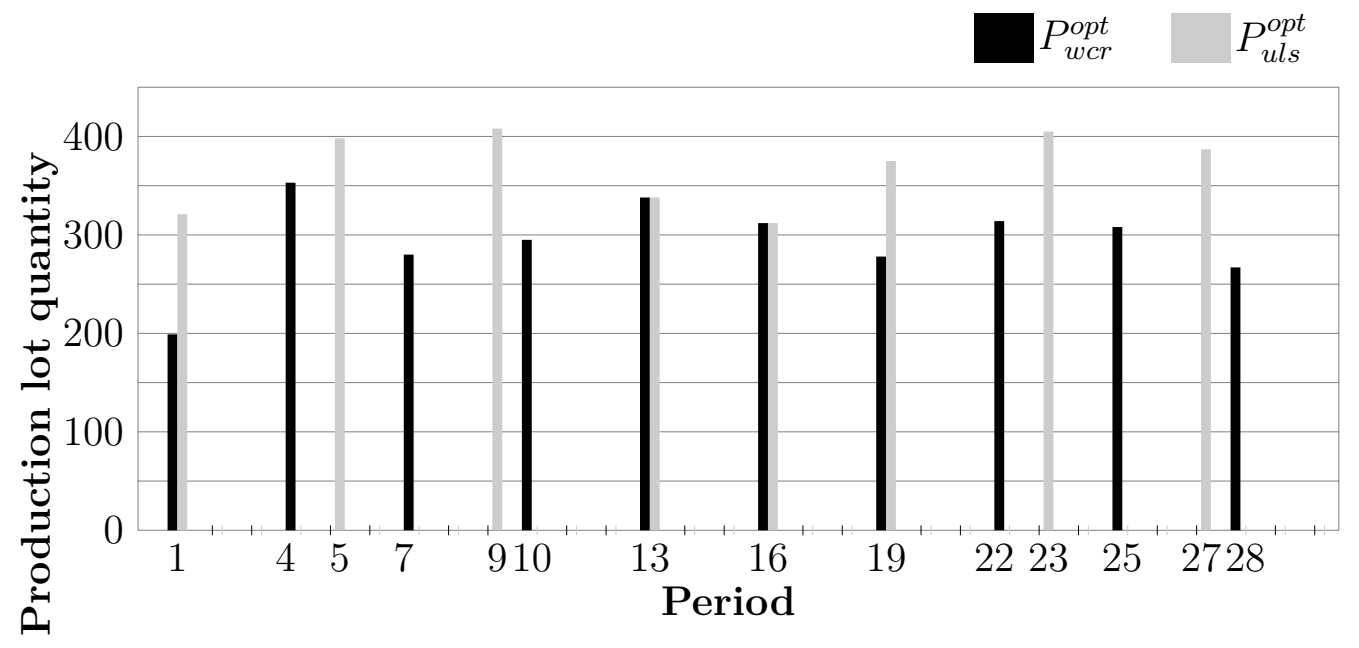

Figure 4.3 - Optimal production program comparison with fixed parameter values

favor the planning with smaller lot size that leads to a smaller inventory holding cost as well as its financial cost.

\subsubsection{Production program evolution with different rates of discount and interest}

In order to illustrate the influences of the two financial aspects (time value of money and financing cost of OWCR) on a production program, the discount and interest rates are respectively varied from 0.01 to 0.1 in steps of 0.01 . The result of the comparison is presented in Figure 4.4. The bold grid represents the numbers of setups in optimal programs proposed by $U L S_{P(W C R)}$ model and the light grid shows the number of setups in optimal programs in the ULS case (which remain unchanged). In this figure, observations can be summarized as follows:

- When the rates are very small, we obtain the same production program with $U L S_{P(W C R)}$ as the optimal ULS program;

- In other cases, the $P_{w c r}^{o p t}$ are different from $P_{u l s}^{o p t}$. Two more production lots may be planned for the financial model when the rates are relatively large.

Consequently, we first deduce from the second observation that considering the financial aspects radically changes the production program from the traditional ULS program which only optimizes logistic costs. Next, $P_{w c r}^{o p t}$ generally proposes more setups which means less products held in inventory during the planning horizon compared with the ULS case. This decrease of average inventory level is numerically presented in Table 4.9 comparing the average inventory level with $P_{u l s}^{o p t}$. For each rate combination, the difference in numbers of setups (\#Setup) and average level of inventory in percentage, $\Delta A v g$. Stock $=\frac{I n v_{w c r}-I n v_{u l s}}{I n v_{u l s}} \times 100 \%$, as presented in Table 4.9

The difference between optimal programs becomes more significant when the financial rates increase. A large decrease of $26 \%$ of average inventory level can be reached with relatively high discount and interest rate according to these tests. Essentially, the financing cost of inventory holding cost is quickly enlarged due to the cumulative effect of a higher inventory level. As a consequence, it is undesirable to hold too much inventory when seeking to maximize the NPV of profit i.e., more frequent setups are preferred. 


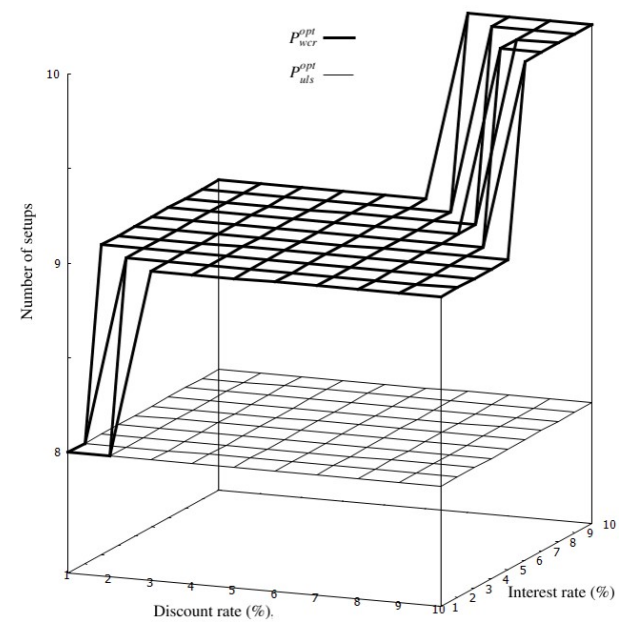

Figure 4.4 - Comparison of number of setups between optimal plans with different interest rates

Table 4.9 - Differences in number of setups and average inventory level between optimal programs of $U L S_{P(W C R)}$ and $U L S$

\begin{tabular}{c|c|c|c|c|c}
\multicolumn{2}{c|}{ Parameter } & $\beta=0.01$ & $\beta=0.02$ & $\beta=0.08$ & $\beta=0.1$ \\
\hline \multirow{3}{*}{$\alpha=0.01$} & \#Setup & $=$ & $=$ & +1 & +1 \\
\cline { 2 - 6 } & $\Delta$ Avg.Stock & 0 & 0 & $-14.2 \%$ & $-14.2 \%$ \\
\hline \multirow{3}{*}{$\alpha=0.02$} & \#Setup & $=$ & +1 & +1 & +1 \\
\cline { 2 - 6 } & $\Delta$ Avg.Stock & 0 & $-14.2 \%$ & $-14.2 \%$ & $-14.2 \%$ \\
\hline \multirow{3}{*}{$\alpha=0.08$} & \#Setup & +1 & +1 & +1 & +2 \\
\cline { 2 - 6 } & $\Delta$ Avg.Stock & $-14.08 \%$ & $-14.08 \%$ & $-14.08 \%$ & $-26.7 \%$ \\
\hline \multirow{2}{*}{$\alpha=0.1$} & \#Setup & +1 & +1 & +2 & +2 \\
\cline { 2 - 6 } & $\Delta$ Avg.Stock & $-14.08 \%$ & $-14.08 \%$ & $-26.32 \%$ & $-26.32 \%$ \\
\hline
\end{tabular}

\subsubsection{Production and financial cost comparisons}

Within these tests, we find 3 cases where $P_{u l s}^{o p t}$ and $P_{w c r}^{o p t}$ are identical when the rates are small. For other cases, we compare the NPV of total costs including the financing cost of OWCR generated by $P_{u l s}^{o p t}$ and $P_{w c r}^{o p t}$, denoted respectively as $N P V_{u l s}$ and $N P V_{w c r}$. Since $P_{w c r}^{o p t}$ generates a minimum NPV of total cost, the increase of NPV in applying the $P_{u l s}^{o p t}$ is computed as $\Delta_{N P V} U L S=$ $\frac{N P V_{u l s}-N P V_{w c r}}{N P V_{w c r}} \times 100 \%$. The result is presented in Figure $4.5\left(\Delta_{N P V} U L S_{P(W C R)}\right.$ represents the minimum NPV of total cost with $P_{w c r}^{o p t}$. $P_{u l s}^{o p t}$ generates a lower NPV of total cost by at most $3 \%$ compared to the NPV obtained by $P_{u l s}^{o p t}$.

On the other hand, because the production cost generated by $U L S$ model, denoted as $\log _{u l s}$ is already optimal, the production cost generated by applying $P_{w c r}^{o p t}$ (denoted as $\log _{w c r}$ ) is logically higher. The increase is calculated as $\Delta_{L O G} U L S_{P}(W C R)=\frac{\log _{w c r}-\log _{u l s}}{\log _{u l s}} \times 100 \%$ and presented in Figure 4.6 .

\subsubsection{Program evaluation with different purchasing costs and delays in payment to supplier}

Considering the delay in payment to supplier only affects the purchasing cost, we thus combine the tests of these two parameters, see Figure 4.7. In these tests, we set the purchasing unit cost varying between 0 to 90 in step of 10 . Moreover, the delay in payment to supplier varies from 0 to 20 in step of 2. Moreover, the rates are fixed at 3\%. First, we observe that following the increase 


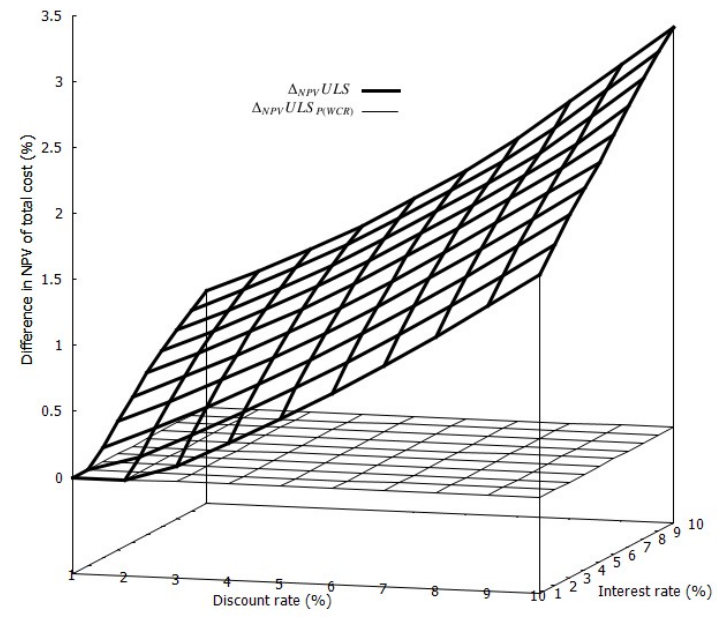

Figure 4.5 - Difference in NPV of total cost including the financing cost



Figure 4.6 - Difference in logistic cost

of purchasing unit cost and with a fixed delay, more production lots are launched which signifies less products held in inventory. The reason is when the purchasing cost is more expensive, we are interested to launch the production later considering the discount effect which makes early activities more costly in term of NPV. Second, with a fixed purchasing unit cost and an increasing delay to supplier, the production size will generally increase for keeping more NPV of inventory value in the system and cash back to the supplier as last as possible.

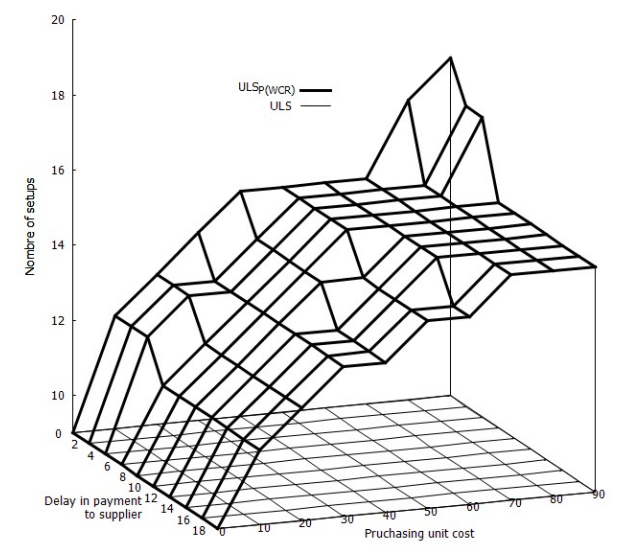

Figure 4.7 - Production plan comparison with varying purchasing unit cost and downstream delay



Figure 4.8 - Variation of number of setups with varying production unit cost

\subsubsection{Program evaluation with different production unit costs}

Based on the parameter value given in Table 4.8, we set the production unit cost at 5 and the rates at $3 \%$. The result is presented in Figure 4.8. The production lot size will decrease with an increasing production unit cost. It is because when the production cost increase, we have less interest to significantly advance the production in an early period considering the discount effort and increased number of periods to finance. 


\subsubsection{Program evaluation with different delays in payment from client}

Similar tests are performed with the rates set at 5\%. Moreover, the purchasing and production unit cost are equal to 10 . Then, we vary the delay in payment from client from 0 to 20 in step of 2 . The result is shown in Figure 4.9. We observe that increasing the delay in payment from client causes a larger the production lot size. The reason is to postpone the production periods for manufacturing the demands and for paying the associated costs in later periods of horizon.

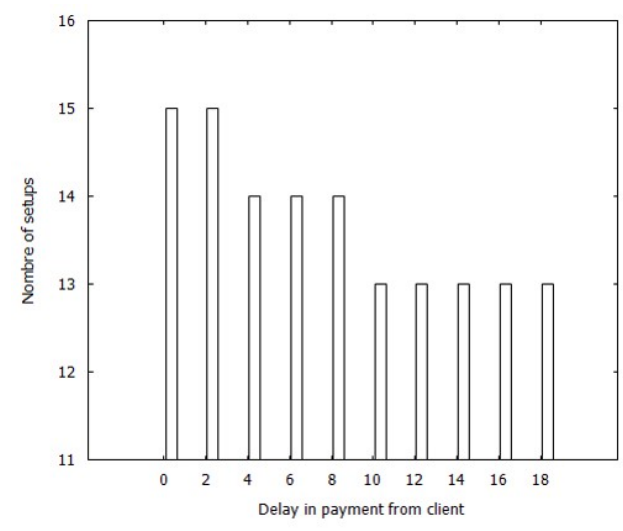

Figure 4.9 - Production plan comparison with varying delays in payment from client

\subsection{Conclusions}

In this chapter, firstly, the generic model of WCR is reformulated to adopt the discrete time horizon. Such a model allows us to measure the evolution of OWCR over the discrete-time planning horizon. Secondly, a ULS-based profit maximization model is developed considering both the financing cost of the WCR and the time value of money. This model is established for the single-site, single-level, single-product and infinite production capacity case. After proving the Zero-InventoryOrdering property for this problem, an exact method is developed based on dynamic programming. With this polynomial algorithm, we are able to not only reach the optimal plan which maximizes the net present value of profit, but also evaluate the profitability of satisfying a series of demands. Numerical tests are provided to show the interest of applying our WCR model through a comparison with the classical $U L S$ model. It is found that we have less interest in holding products in the inventory due to their dramatic amplification of financial costs. In addition, we are aware that this model has limitations as the first effort which theoretically considers the WCR cost in the classic dynamic lot-sizing problem. Immediate application for practical problem is still difficult. However, it will contribute to further studies in more complex and realistic cases as a polynomial subproblem.

For future research, it seems promising to reduce the complexity of this algorithm to $\mathrm{O}\left(T^{3} \log T\right)$ by examining the approaches presented in Wagelmans et al. (1992), Aggarwal \& Park (1993) and Federgruen \& Tzur (1991) that improve the complexity of the Wagner-Whitin algorithm. Moreover, future research can be undertaken to consider time-varying cost parameters for this case, such as the Wagner-Whitin cost in the case of the ULS. With this cost, the complexity of the fast backward dynamic programing algorithm of Wagelmans et al. (1992) can be improved from $O(T \log T)$ to $O(T)$. In order to establish a global profitability evaluation, a two-level (customer-factory) profit maximization problem will be addressed in following chapter. Furthermore, the production capacity and risks in supply chain management should be taken into account among other extensions. 


\section{Multi-level uncapacitated lot sizing based discounted model considering the financing cost of working capital requirement}

\subsection{Introduction}

In this chapter, firstly, we extend the aforementioned single-level model to a two-level (suppliercustomer) model and further generalize it to a multi-level case based on the Multi-level Lot-sizing (MLLP) model. The OWCR model is then modified for adapting to this multi-level scenario. Considering the OWCR cost in multi-level case, we propose sequential and centralized approaches to solve both the two-level and the multi-level problem with a serial chain structure. The ZIO property still unaffectedly stands in the sequential approach and is further proven valid in the centralized approach. The property allows us to establish a dynamic programming algorithm which solves the problem in $O\left(N T^{6}\right)$ (where, $L$ represents the number of level in the considered supply chain). Finally, we analyze the disparities in the optimal solutions obtained by both the sequential and the centralized approaches.

\subsection{The multi-level uncapacitated lot sizing-based models}

\subsubsection{MRP and the multi-level lot-sizing problem}

Since the introduction of materials requirement planning (MRP) in the late 1960's, companies have rapidly implemented it when facing increasing customer needs (Davis (1975)). This situation requires manufacturing production systems to deal with larger and more complex product structures. This evolution has magnificently revealed the significance of the MLLP problem in the MRP process. The principal concept is to translate the production program of a finished item into the programs of its components. Those programs are calculated on the basis of the bill-of-material (BOM) and leadtime information. Nevertheless, several difficulties and limits of the MRP are revealed in practice that restricts the use of MRP setting:

- No capacity constraints considered is MRP planning. 
- Unpredictable demands of finished items and production lead times deeply affect the accuracy of the planning with MRP.

- Integrity of the data considerably impacts the accuracy of the result.

First, in the fundamental concept of the MRP system, capacity constraints are not considered. No limit of production quantity restraints the planning at all levels. Consequantly, the feasibility of the result obtained by the MRP system can thus not be guaranteed. Moreover, the leeway of operation capacity can also be used for reallocation at the practical level via the shop floor based on initial plan obtained by MRP.

Second, demand uncertainty causes the difficulty of accurately estimating customer demands in the years to come. This problem leads to a significant challenge of inventory control and management. Indeed, uncertain demands may result from a variety of reasons. For instance, demands of seasonal merchandises may significantly fluctuate over time and sometimes strongly correlate with a particular event. Moreover, changes in trade credit terms and customer's needs, and alteration in technology development and situation of competitors can influence the demands as well. The bull-whip effect is a common result of the demand fluctuation resulting in an important adjustment especially required in the upstream of the supply chain.

Furthermore, in a tactical production planning context, the longer the lead time, the higher the level of total inventory. The lead time, computed in days, is typically the sum of the supply delay, that is to say, the time delay between placing order and demand delivery incorporating the transit time, and the reordering delay, which is the time until an ordering opportunity arises again.

Third, if there exists any errors in the inventory data, the bill of materials (commonly referred to as 'BOM') data, or the master production schedule, the output data will then be incorrect.

Despite all this restrictions, the MRP yet have been largely implemented for the production planning. However, for a direct application of the MRP planning, all calculations should be repeated for each adjustment in accordance with demand or lead time changes. To meet the requirement, simple "heuristic" solutions have been developed. In this chapter, we address the multi-site lot sizing problem with a serial supply chain structure while taking into account the financing cost of the OWCR. In this problem, we exclusively consider the transport delay, without the consideration of the transport cost. According to the comparison provided in Lemoine (2008), the multi-level models can be applied in our case where the transport aspect is overlooked. In the following paragraph, the problem description, mathematical model and solution procedure are put forward.

\subsubsection{The description of the MLLP problem and associated mathemat- ical formulation}

The problem of the multi-level production planning considers products made from several other components. Specifically, finished products require components for which it is also necessary to determine the corresponding production plans. These components may also have subcomponents, etc. Meaning that different levels of BOM are taken into account, hence the term "multi-level". In these models, we thus find the two types of demand (external and internal) and the internal demand is deduced from the external demand according to the BOM structure.

Generally speaking, the BOM is represented by an acyclic graph (as in Bookbinder \& Koch (1990)) where the nodes represent the products (or its components) and the arcs stand for the links between the different nodes. For each finished product $i$, one can list all the products $j$ directly involved in its manufacture and the technical coefficient $r_{i j}$ is defined as the quantity of product $i$ directly used to manufacture a unit of product $j$. The valued graph is named "graph gozinto". The acyclic character of the graph allows it to be split into several levels. By convention and based on the definition of the product BOM, the products of level 0 are the finished products. However, they merely represent the external demand. If a product $i$ is at level $n$, all products directly involved in its manufacture will be at level $n+1$. This graph can also be formulated using a square matrix 

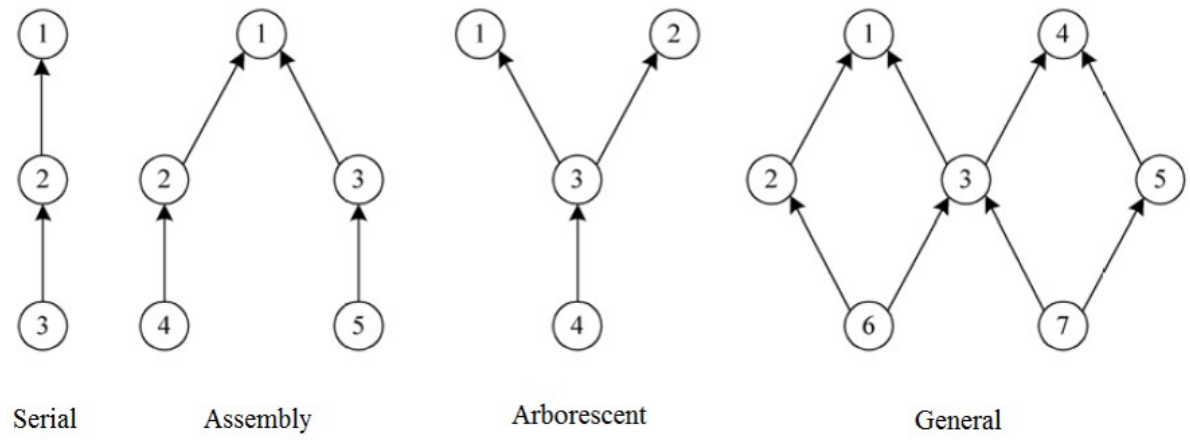

Figure 5.1 - Four product BOM structures

called the "gozinto matrix". Its dimensions are equal to the number of products to be planned for production, and where each element $r_{i j}$ represents the number of products $i$ directly involved in the manufacture of the product $j$. Since the graph is acyclic, the matrix can be represented in a lower triangular form with a diagonal of zero.

In the literature, there are four types of structure (gozinto graphs) illustrated in Figure 5.1 .

- the structure in series: each product has at most a predecessor and at most one successor,

- the assembly structure: each product has at most one successor,

- the arborescent/tree structure: each product has at most a predecessor,

- the general structure where there is no limitation on the number of all predecessors and successors.

In the literature, the MLLP model is built for the multi-level, big bucket and infinite capacity case to satisfy time-varying demand. The mathematical formulation can be found in Steinberg \& Napier (1980) and Clark \& Armentano (1995) and the latter is presented below. The parameters and decision variables are given in Table 5.1 and 5.2 .

\begin{tabular}{ll}
\hline Parameter & Definition \\
\hline$N$ & number of levels, \\
$S(i)$ & number of periods, \\
$P(i)$ & the set of all immediate successor components to component $i$, \\
& the set of immediate predecessors of component i; if $\mathrm{P}(\mathrm{i})=\emptyset$, then $i$ \\
$r_{i j}$ & is called end item, \\
& the number of units of $i$ required for production of one unit of component \\
$d_{i t}$, & $j \in S(i)$. \\
$p_{i t}$ & the independent demand for component $i$ in period $t$, \\
$s_{i t}$ & the unit production cost of component $i$ in period $t$, \\
$h_{i t}$ & the fixed setup cost occurred if component $i$ is produced in period $t$, \\
$M$ & the unit holding cost of component $i$ at the end of period $t$, \\
$L(i)$ & upper bound of possible production lot-size, \\
$T(i)+1$ & the production lead time of component $i$, in unit of periods, to ensure the \\
& lot $Q_{i t}$ is available for consumption only at the beginning of period $t+L(i)$, \\
& starts over the $T$ periods from $T(i)+1$ to $T(i)+T$ in order to synchronize its \\
& production with that of the other components, in particular with that of \\
& the finished item.
\end{tabular}

Table 5.1 - Parameters 


\begin{tabular}{ll}
\hline Variable & Definition \\
\hline$Q_{i t}$ & production lot-size of component $i$ in period $t$, \\
$Y_{i t}$ & binary variables equal to 1 if component $i$ is produced in period $t$ and 0 if not, \\
$I_{i t}$ & the quantity of component $i$ held in inventory at the end of period $t$, \\
\hline
\end{tabular}

Table 5.2 - Decision variables

Thus, the mathematical formulation can be written as below:

$$
\begin{array}{ccl}
\text { Min } & \sum_{i=1}^{N} \sum_{t=T(i)+1}^{T(i)+T}\left[s_{i t} Y_{i t}+p_{i t} Q_{i t}+h_{i, t+L(i)} I_{i, t+L(i)}\right] \\
\text { s.t. } & I_{i, t+L(i)-1}+Q_{i t}-I_{i, t+L(i)} & \\
=d_{i, L(i)+t}+\sum_{j \in S(i)} r_{i j} Q_{j, L(i)+t} & \forall i \in[1, N] \\
& & \forall t \in[T(i)+1, T(i)+T] \\
M Y_{i t}-Q_{i t} \geq 0 & \forall(i, t) \in[1, N] \times[1, T] \\
Q_{i t}, I_{i, t+L(i)} \geq 0 & \forall i \in[1, N] \\
Y_{t}=0 \text { or } 1 & \forall t \in[T(i)+1, T(i)+T]
\end{array}
$$

The objective is to minimize the sum of the costs of setup, production and holding to satisfy all the demands over the planning horizon. It is noted that the production of each component $i$ takes place on a rolling horizon. Constraints (4.2) represent the equilibrium of inventory in each period. Constraints (4.3) require that a setup for product $i$ be carried out in period $t$ if there is production of it during this period. Constraints (4.4) define the setup variables as binary and (4.5) are non-negativity conditions.

In contrast to the complexity of the ULS model, even though the optimal solution complies with the zero-inventory property (Veinott Jr (1969)), this model is proven NP-hard by Arkin et al. (1989). Optimal solutions can only be obtained in special cases and small size problems, as reviewed in Bahl et al. (1987), Goyal \& Gunasekaran (1990) and Brahimi et al. (2017). In next section, related works are tersely presented based on the BOM structures and types of the solution approach.

\subsubsection{Review of the multi-level problem}

In the MLLP literature, few exact methods have been developed:

- One of the early works can be found in paper of Zangwill (1966) which proposes a dynamic programming (DP) algorithm for the case of a serial BOM adopting flow constraints in a singlesource network. The complexity of this algorithm for $N=2$ is $O\left(T^{3}\right)$ and for $N>2$ is $O\left(N T^{4}\right)$ where $N$ is the number of levels. Based on this work, Love (1972) takes a serial structure problem into consideration under level-related costs assumptions. The author also proposes a dynamic programming algorithm to determine "nested extreme optimal schedule". In such a schedule, the production of an item and of all its successors are in the same period. In the extension of the previous work, Crowston \& Wagner (1973) develop dynamic programming and Branch \& Bound (B\&B) methods for assembly systems. Moreover, Steinberg \& Napier (1980) consider commonality of items and propose a Mixed Integer Programming (MIP) formulation of a constrained generalized network with fixed charge arcs and side constraints. This work serves as a benchmark to evaluate heuristic algorithms and is limited to small size problem.

- Furthermore, Afentakis \& Gavish (1986) focus on the general structure BOM by transforming complex structures into assembly ones whose optimal solutions can be efficiently computed (Afentakis et al. (1984)). In their work, MLLP model has been reformulated by using the notion of "echelon stock" initially introduced by Clark \& Scarf (1960). It allows us to simplify the stock equilibrium constraint in the MLLP model (i.e. constraints (4.2)) by replacing the 
terms of internal demand. This reformulation makes the constraint in a similar form to the ones of the single-level models and allows us to build a strong single-item relaxation which leads the authors to an efficient B\&B algorithm using Lagrangian relaxation. More relevant early works are summarized in Dellaert \& Jeunet (2003) and Pochet \& Wolsey (2006).

- Recently, Van Hoesel et al. (2005) give a DP algorithm for two-level and multi-level problems in which constant capacity constraint merely exists in the initial production level. Melo \& Wolsey (2010) consider the uncapacitated two-level problem with serial structure and develop a DP algorithm that runs in $O\left(T^{2} \log T\right)$ using the approach of Eppen \& Martin (1987). For the same model but with intermediate independent demand, Zhang et al. (2012) give a partial description of the convex hull of solutions in the original variable space. Besides, the authors develop a polynomial-time dynamic programming algorithm, which leads to a tight and compact extended formulation.

In addition to those exact methods, considerable efforts have been continuously invested for developing heuristic and meta-heuristic methods over decades in order to tackle the computational difficulty. In the literature, these methods can be classified into three main types:

- Sequential approach, which determines the production program level by level following the MRP logic.

- Simultaneous (centralized) approach, with a global optimization objective including simultaneously all levels.

- Decentralized or distributed approach, which considers an interaction between the actors of the supply chain.

The sequential approach is the one of the first methods developed for the multi-level problem in the MRP framework which decomposed the multi-level problem into polynomial single-level problems, for instance, with the Wagner-Whitin algorithm (Veral \& LaForge (1985)). States of the art on various related methods are provided in Bahl et al. (1987) and Gupta \& Keung (1990). The basic idea of this approach is to consider the optimal production program calculated for the upper level as the demand to satisfy for the adjacent lower level. The direct application of the single-level method after the decomposition in case of complex product structure may induce unnecessary high setup and holding costs because the interdependence between levels has been ignored. In the literature, cost modification (revision) is proposed for taking into account some degree of interdependence of demands between levels. This demand interdependence stems from the fact that planning a production of item $i$ would possibly trigger the production of its components that may cause an additional setup cost. In some work, the cost modification also contains the holding cost. In these revisited costs, the single-level method is used to each item in the product structure. Blackburn \& Millen (1982) compare five types of modifications for setup and inventory holding costs in case of the single end-item assembly system. Bookbinder \& Koch (1990) later extend this cost revision type method for more general product structure cases. Recent work of Dellaert \& Jeunet (2003) pursue ideas of early cost modification methods and give a randomized MLLP heuristic for general product structure. Two main contributions of this work are considering time-varying setup cost and randomizing their algorithm multiplying the modification term with Monte Carlo simulation. More related methods are also reviewed in this paper.

The simultaneous (centralized) approach, often based on metaheuristic algorithms, has emerged much more recently. Its performance are reported for providing highly cost-efficient solutions with a reasonable computation effort. Kuik \& Salomon (1990) implement simulated annealing for six neighborhood systems and compare their effectiveness. Dellaert \& Jeunet (2000) and Dellaert et al. (2000) propose a hybrid genetic algorithm (HGA) for this problem with a general product structure. In those works, a competitive strategy is introduced to mix five operators in the chromosome evolutions between consecutive generations. Tang (2004) raises a novel neighborhood system based on a property called "Inner corner" and develop a simulated annealing algorithm to handle serial structure problems. Jeunet \& Jonard (2005) also propose a simulated annealing based on solution 
coding, the two best performing neighborhood systems given by Kuik \& Salomon (1990) and three new neighborhood systems. Homberger (2008) present a parallel genetic algorithm (PGA) with an empirical policy for subpopulations migrations. This method benefits from sharing calculation load over multiple processors. Other metaheuristics algorithms, such as the particle swarm optimization (PSO) algorithm by Han et al. (2009) and Deroussi \& Lemoine (2009), the ant colony optimization (ACO) system by Pitakaso et al. (2007) and Homberger \& Gehring (2009), the segmentation-based soft optimization approach (SOA) by Kaku et al. (2010) and variable neighborhood search (VNS) by Xiao et al. (2014), have been proposed for solving the MLLP problems.

Third, in the MLLP model, we implicitly assume that only one decision maker who decides the production planning for all items with all necessary information on cost parameters exists. This assumption is normally fulfilled within a single company. Nevertheless, in practice and in a global supply chain, the components are often supplied by different independent companies (Christopher (1999)). Hence, this assumption is not practicable in some cases (Dudek \& Stadtler (2005), Straube \& Beyer (2006)), i.e. no decision maker is authorized to make production decisions for the entire supply chain. Distributed (Decentralized) approaches are developed to fill this gap in MLLP context, for instance, in the recent work of Homberger (2010), Buer et al. (2013) and Homberger et al. (2015) with different negotiation mechanisms.

In addition, the NPV principle has been identified as a natural approach to reveal the economic consequences in MRP theory in the early work of Grubbström (1967). The author shows that cash flows can be immediately transformed by exchanging the Laplace frequency for the continuous interest rate. The author and his colleagues have made an significant contribution to the MRP theory in view of economic aspect while using NPV approach, input-output analysis and Laplace transform with a continuous-time model. Related works are referred to Grubbström (1998), Grubbström (1999), Bogataj \& Grubbström (2012) and other works in its references. Furthermore, discrete-time models with consideration of the NPV have also been developed in the literature. Helber (1998) treats the multi-level problem in the framework of Manufacturing Resource Planning (MRP II) with multiple capacity constraints and setup times in a discrete-time horizon. Grubbström \& Wang (2003) present a model of multi-level capacity-constrained system with a stochastic external demand and a constant lead time for maximizing expected NPV of profit. A dynamic programming procedure is applied to solve the problem. Based on the previous work, Grubbström \& Huynh (2006) establish a similar model with deterministic demand and extend the model for any non-zero lead time. Other model considering the NPV approach also exists in multi-site models which are reviewed in Martínez-Costa et al. (2014).

In this chapter, we extend our single-level model to two-level, and then to multi-level with a serial chain structure. The corresponding OWCR model is presented for multi-level case and the associated non-linear Mix Integer Programming (MIP) formulation with an objective to maximize the NPV of profit is given. To study the structural property of optimal planning, we first prove the Zero-Inventory-Ordering (ZIO) property in the two-level case, and then we generalize it to the multi-level problem. With this property, dynamic programming algorithms are developed for both cases. The financial consequences of the operation decisions on the production programs are analyzed through numerical tests.

\subsection{Two-level MLLP discounted models considering OWCR financing cost}

\subsubsection{Problem description}

This problem is named as $2 U L S_{P(W C R)}$ problem in which we consider a supply chain composed of two sites which extends the previous single-level model. In this system, the supplier purchases 
and process the raw material to produce intermediate goods in site $S_{0}$. These goods are delivered to the manufacturer for further processing in site $S_{1}$ in order to satisfy the external demand. Only one type of item is manufactured in each site which are respectively denoted as $P_{0}$ in $S_{0}$ and $P_{1}$ in $S_{1}$. More assumptions are presented in the paragraphs below.

Each site is characterized by:

- logistic costs: purchasing costs (noted $a_{i}, i \in\{0,1\}$ ), setup costs (noted $s_{i}, i \in\{0,1\}$ ), production costs (noted $p_{i}, i \in\{0,1\}$ ) and inventory holding costs (noted $h_{i}, i \in\{0,1\}$ ). According to common sense, the purchasing unit cost of the manufacturer should be higher than the total unit cost for its production in supplier (i.e. including all four type of costs);

- term of payment: one of the differences compared to the single-level case is found in the payment delay between the site $i-1$ and site $i$ (denoted as $r_{i}$ ). In this two-level case, the supplier will receive the payment from the manufacturer after $L_{1}+r_{1}$ periods which includes both the delivery and payment delays between these sites. Furthermore, no particular index is given for the final customer who command the external demand and the tier 2 supplier who provides the raw material. The payment delay from the final customer to the manufacturer is denoted as $r_{2}$ and the payment from the supplier to the tier 2 supplier is denoted as $r_{0}$. Moreover, the delivery delays of raw martial and finished product are not considered in this problem, i.e. $L_{0}=L_{2}=0$.

- financial aspects: the same financial aspects (discount and interest rate) as in the previous single-level model are considered in this case. The discount rate is denoted as $\alpha_{i}, i \in\{0,1\}$ and the interest rate (the rate of financing the WCR) are denoted as $\beta_{i}, i \in\{0,1\}$.

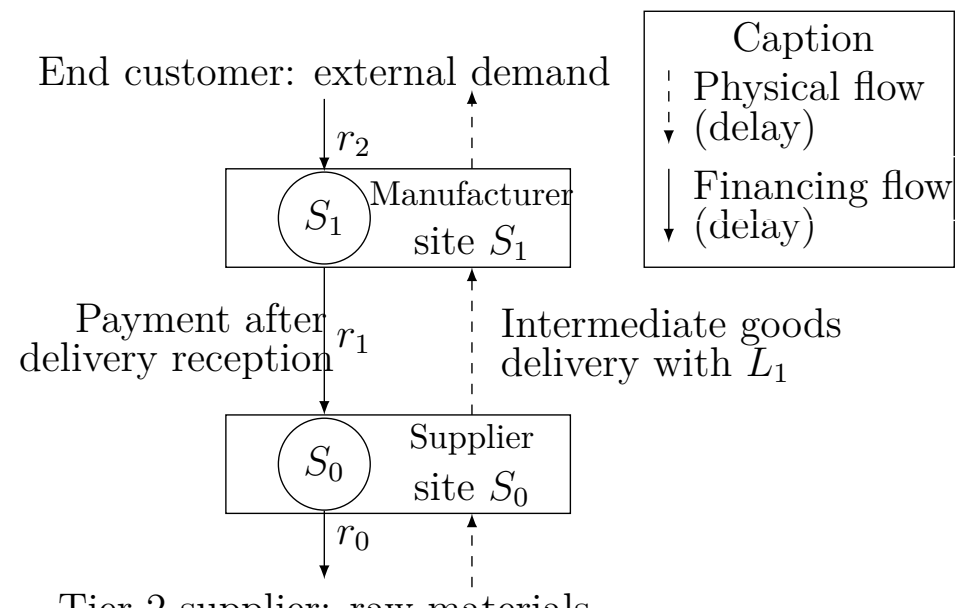

Tier 2 supplier: raw materials

Figure 5.2 - Supply chain and delay

According to Hofmann et al. (2011) (chapter5, pp64), buying company often has a lower capital cost rate than its supplier resulted from a shortened cash-to-cash cycle. Moreover, in practice, the unit sales price of supplier (i.e. the unit purchasing cost of manufacturer) is usually greater than average cost per product including all logistic and financial cost in order to gain profit. Furthermore, "The supplier frequently has a lower inventory carrying rate since its goods are warehoused in large quantities and scaling effects are achieved." (Hofmann et al. (2011), chapter5, pp63). Thus, the inventory holding cost is greater in $S_{1}$ than in $S_{0}$ (i.e. $h_{0}<h_{1}$ ). In addition, we assume the $r_{1} \geq r_{2}$ for this problem which is the case where the manufacturer take the dominant plan in the chain. We also assume that the interest rates are equal in the centralized approach. To conclude, the two-level model is established under the following assumptions:

- Production aspects:

- No replenishment and production delays as they are negligible compared to the period duration; 
- Demand should be met on time (no backlogging);

- Initial and final stock of all items are defined as zero;

- One unit of item is manufactured using one unit of component or raw material;

- Delivery delays of raw material and finished product are not considered;

- Intermediate goods are only held in the inventory of the supplier;

- Inventory holding unit cost is greater in the manufacturer level than the one in the supplier level.

- Financial aspects:

- The payment of the manufacturer to the supplier for the intermediate goods is executed after receipt of the goods;

- Payments of all logistic and financial costs are made at the beginning of periods;

- The margin resulting from the sale of selling products will not be used for financing the WCR;

- Products in the same lot uniformly share the setup cost. However, the inventory holding cost is measured for each product according to its total holding time in the inventory;

- The purchasing unit cost of one level should be higher than the unit cost for producing one unit at the lower (adjacent) level;

- The discount rate is greater at the supplier level than the one of the manufacturer level;

- The payment delay from the manufacturer is shorter or equal to the one from the supplier;

- The interest rates of the two levels are assumed equal in the centralized approach.

\subsubsection{OWCR model in two-level case}

The notations of parameters and decision variables are similar to the single-level model and adapted for the two-level case, see Table 5.3 and 5.4. In the two-level case, $i$ can take three values, 0 for the supplier and 1 for the manufacturer ( 2 for the external customer).

\begin{tabular}{ll}
\hline Parameter & Definition \\
\hline$T$ & Horizon length \\
$d_{i t}$ & Demand in period $t$ for site $i$ \\
$v_{i}$ & Unit selling price \\
$h_{i}$ & Unit inventory cost per period \\
$p_{i}$ & Unit production cost \\
$s_{i}$ & Fixed setup cost \\
$a_{i}$ & Unit purchasing cost \\
$r_{i}$ & Delay in payment from site $i$ to site $i-1$ \\
$L_{i}$ & Delivery lead time of intermediate goods from site $i-1$ to site $i$ \\
$\alpha_{i}$ & Discount rate per period of site $i$ \\
$\beta_{i}$ & Interest rate for financing OWCR per period of site $i$ \\
\hline
\end{tabular}

Table 5.3 - Parameters for OWCR modeling in two-level case

\begin{tabular}{ll}
\hline Variable & Definition \\
\hline$Q_{i t}$ & Total production quantity in period $t$ of site $i$ \\
$X_{i t k}$ & Production quantity in period $t$ for satisfying (a part of) demand in period $k$ of site $i$ \\
$I_{i t}$ & Product quantity held in inventory in period $t$ of site $i$ \\
$Y_{i t}$ & Binary variable which indicates whether a setup occurs in period $t$ or not in site $i$ \\
\hline
\end{tabular}

Table 5.4 - Decision variables for OWCR modeling in two-level case

At each period, the WCR is composed of the same terms as the single-level case, for covering the purchasing (purch), setup (setup), production ( prod) and inventory holding (inv) costs. The 
formulation of the WCR in this case is similar to the previous case for covering the different logistic costs associated to the disaggregated production quantity, $X_{i t k}$ (where $i \in\{0,1\}, t \in[0, T]$ and $k \in[t, T])$. For instance, $W C R_{i t k}^{\text {purch }}$ represents the WCR for financing the purchasing cost related to $X_{i t k}$. In the general formulation proposed thereafter, we consider the beginning of the supplier's production as the beginning of the global planning horizon. For this reason, the delivery delay to site $i$ will postpone its planning in the global planning horizon and add additional discount effects to the incoming and outgoing cash flows of site $i$. Therefore, for instance, the first term in the formulation of the manufacturer's WCR, $\frac{1}{\left(1+\alpha_{i}\right)^{L_{1}}}$ reflects the discount effect of this backward shifting of planning horizon for $L_{1}$ periods. The WCR of the supplier and of the manufacturer are respectively formulated with the following equations, where $i \in\{0,1\}, t \in[0, T]$ and $k \in[t, T]$.

Supplier's WCRs are written as:

$$
\begin{gathered}
W C R_{0 t k}^{\text {purch }}=a_{0} \cdot X_{0 t k} \cdot\left(\sum_{j=t+r_{0}}^{T} \frac{1}{\left(1+\alpha_{0}\right)^{j}}-\sum_{\substack{j=k+L_{1} \\
+r_{1}}}^{T} \frac{1}{\left(1+\alpha_{0}\right)^{j}}\right) \\
W C R_{0 t k}^{\text {prod }}=p_{0} \cdot X_{0 t k} \cdot \sum_{j=t}^{+r_{1}-1} \frac{1}{\left(1+\alpha_{0}\right)^{j}} \\
W C R_{0 t k}^{\text {setup }}=\frac{s_{0} \cdot Y_{0 t}}{Q_{0 t}+1-Y_{0 t}} \cdot X_{0 t k} \cdot \sum_{j=t}^{+L_{1}-1} \frac{1}{\left(1+\alpha_{0}\right)^{j}} \\
W C R_{0 t k}^{i n v}=h_{0} \cdot X_{0 t k} \cdot \sum_{w=t}^{k-1} \sum_{j=w}^{k+r_{1}-1} \frac{1}{\left(1+\alpha_{0}\right)^{j}}
\end{gathered}
$$

Manufacturer's WCRs are formulated as:

$$
\begin{gathered}
W C R_{1 t k}^{\text {purch }}=\frac{1}{\left(1+\alpha_{1}\right)^{L_{1}}} \cdot a_{1} \cdot X_{1 t k} \cdot\left(\sum_{j=t+r_{1}}^{T} \frac{1}{\left(1+\alpha_{1}\right)^{j}}-\sum_{j=k+r_{2}}^{T} \frac{1}{\left(1+\alpha_{1}\right)^{j}}\right) \\
W C R_{1 t k}^{\text {prod }}=\frac{1}{\left(1+\alpha_{1}\right)^{L_{1}}} \cdot p_{1} \cdot X_{1 t k} \cdot \sum_{j=t}^{r_{2}-1} \frac{1}{\left(1+\alpha_{1}\right)^{j}} \\
W C R_{1 t k}^{\text {setup }}=\frac{1}{\left(1+\alpha_{1}\right)^{L_{1}}} \cdot \frac{s_{1} \cdot Y_{1 t}}{Q_{1 t}+1-Y_{1 t}} \cdot X_{1 t k} \cdot \sum_{j=t}^{r_{2}-1} \frac{1}{\left(1+\alpha_{1}\right)^{j}} \\
W C R_{1 t k}^{i n v}=\frac{1}{\left(1+\alpha_{1}\right)^{L_{1}}} \cdot h_{1} \cdot X_{1 t k} \cdot \sum_{w=t}^{k-1} \sum_{j=w}^{k+} \frac{1}{\left(1+\alpha_{1}\right)^{j}}
\end{gathered}
$$

Combining all these terms, the total WCR for the site $i \in\{0,1\}$ is given by the eq. 5.9

$$
W C R_{i}=\sum_{t=1}^{T} \sum_{k=t}^{T}\left(W C R_{i t k}^{\text {purch }}+W C R_{i t k}^{\text {prod }}+W C R_{i t k}^{\text {setup }}+W C R_{i t k}^{i n v}\right)
$$

and the financing cost of $W C R_{i}$ is thus $\beta_{i} \times W C R_{i}$. 


\subsection{Proposed approaches}

With the WCR model for the two-level problem, we develop the two traditional approaches as presented in the Section 5.2.3. the sequential and the centralized approaches:

1. A sequential approach based on the resolution of two independent single-level problems with a MRP-like logic and the maximization of the manufacturer's profit is tackled as a matter of priority;

2. A centralized approach that consists to solve the global problem that maximizes the sum of profits of both sites;

Since the constraints in these two approaches are the same as those adopted from the classical MLLP model presented in the Section 5.2.2. The only difference is on the objective function formulated as in the Section 5.4.1.

\subsubsection{Mathematical formulation of objective functions in sequential and centralized approaches}

The objective functions of theses approaches mainly depend on the total logistic and financial costs and the profit of each site. They can be written in a similar form as in the single-level problem. The formulations of the logistic cost for each site are composed of four components over the entire horizon computed with the following equations. For the supplier,

$$
\begin{gathered}
L C_{0}^{\text {purch }}=a_{0} \sum_{t=1}^{T} \frac{Q_{0 t}}{\left(1+\alpha_{0}\right)^{t+r_{0}}} \\
L C_{0}^{\text {prod }}=p_{0} \cdot \sum_{t=1}^{T} \frac{Q_{0 t}}{\left(1+\alpha_{0}\right)^{t}} \\
L C_{0}^{\text {setup }}=s_{0} \cdot \sum_{t=1}^{T} \frac{Y_{0 t}}{\left(1+\alpha_{0}\right)^{t}} \\
L C_{0}^{\text {inv }}=h_{0} \cdot \sum_{t=1}^{T} \sum_{k=t}^{T} \sum_{q=t}^{k-1} \frac{X_{0 t k}}{\left(1+\alpha_{0}\right)^{q}}
\end{gathered}
$$

For the manufacturer,

$$
\begin{gathered}
L C_{1}^{\text {purch }}=\frac{1}{\left(1+\alpha_{1}\right)^{L_{1}}} \cdot a_{1} \sum_{t=1}^{T} \frac{Q_{1 t}}{\left(1+\alpha_{1}\right)^{t+r_{1}}} \\
L C_{1}^{\text {prod }}=\frac{1}{\left(1+\alpha_{1}\right)^{L_{1}}} \cdot p_{1} \cdot \sum_{t=1}^{T} \frac{Q_{1 t}}{\left(1+\alpha_{1}\right)^{t}} \\
L C_{1}^{\text {setup }}=\frac{1}{\left(1+\alpha_{1}\right)^{L_{1}}} \cdot s_{1} \cdot \sum_{t=1}^{T} \frac{Y_{1 t}}{\left(1+\alpha_{1}\right)^{t}} \\
L C_{1}^{\text {inv }}=\frac{1}{\left(1+\alpha_{1}\right)^{L_{1}}} \cdot h_{1} \cdot \sum_{t=1}^{T} \sum_{k=t}^{T} \sum_{q=t}^{k-1} \frac{X_{1 t k}}{\left(1+\alpha_{1}\right)^{q}}
\end{gathered}
$$

In total, we denote that $L C_{i}=L C_{i}^{\text {purch }}+L C_{i}^{\text {prod }}+L C_{i}^{\text {setup }}+L C_{i}^{\text {inv }}$. The overall revenue of selling the intermediate goods and the finished products are respectively $R_{i}=\sum_{t=1}^{T} v_{i} \cdot d_{i t}$ where $i \in\{0,1\}$. It should notice that $d_{0 t}=Q_{1, t+L_{1}}$, with $\forall t$ and $d_{1 t}$ is the external demand. Consequently, the objective 
function of the sequential approach in this two-level problem is to maximize the manufacturer's profit at first:

$$
\operatorname{Max} \quad R_{1}-\left(L C_{1}+\beta_{1} W C R_{1}\right)
$$

Then, using the obtained production program as the demand for the supplier, we further tackle the profit maximization problem for the supplier:

$$
\operatorname{Max} \quad R_{0}-\left(L C_{0}+\beta_{1} W C R_{0}\right)
$$

Then, the objective function of the centralized approach is written as:

$$
\operatorname{Max} \sum_{i=0}^{1}\left[R_{i}-\left(L C_{i}+\beta_{i} W C R_{i}\right)\right]
$$

For both approaches, the constraints are the same as the ones in classic MLLP problem except for the inventory equilibrium constraints. Ther are reformulated based on the one-to-one serial structure we consider in this problem as in the equation 5.18 .

$$
I_{i, t+L_{i}-1}+Q_{i t}-I_{i, t+L_{i}}=d_{i, L_{i}+t}+Q_{j, L_{i}+t} \quad \forall i \in\{0,1\}
$$

\subsubsection{Sequential approach}

The sequential approach is based on the MRP logic in which products are planned level by level according to the bill of materials. In this approach, the two-level problem could be considered as two independent single-level problems. Thus, the resolution consists in applying twice the single-level algorithm as presented in Figure 5.3.

1. Firstly, the optimal production plan of the manufacturer site $S_{1}$ is calculated with the singlelevel dynamic programming algorithm for satisfying the external demand of item $P_{1}$.

2. Secondly, using the previous optimal plan, the corresponding optimal production plan of the supplier site $S_{0}$ is computed with the same algorithm.

Since the complexity of the single-level algorithm is shown as $\mathrm{O}\left(T^{4}\right)$, the computation time of the sequential is thus $\mathrm{O}\left(T^{4}\right)$.

\subsubsection{Centralized approach}

In a centralized problem, we maximize the global profit of the two levels. In the early work of Zangwill (1966), the author proposed a dynamic programming based algorithm to solve the MLLP problem with serial BOM structure with a computation time of $O\left(N T^{4}\right)$, where $N$ is the number of levels in the problem. Zangwill models the problem in the form of a network with nodes presented as in Figure 5.4. Such a node $(i, j)$ represents the period $i$ in the site at the level $j$. Passing the node, the vertical arcs represent the production quantity (for example, $Q_{i, j}$ is the production quantity in the period $i$ at the level $j$ ); the horizontal arc, $I_{i, j}$, denotes the quantity of products held in inventory in the period $i$ at the level $j$. Therefore, the production decision at the node $(i, j)$ consists in determining the value of $Q_{i, j+1}$ which is for satisfying the demand of the level $j+1$. This decision is made based on the upstream incoming flows, i.e. $Q_{i, j}$ and $I_{i-1, j}$. This decision will also determine the value of the other downstream flow, i.e. $I_{i j}$. With the values of these arcs, we are able to calculate the setup and production cost in function of $Q_{i, j}(\forall i, j)$ and the inventory holding cost in function of $I_{i, j}(\forall i, j)$.

However, the provenances of upstream flows can not be precisely traced in this network representation. Therefore, we can not get the information about the production timing of the products in 


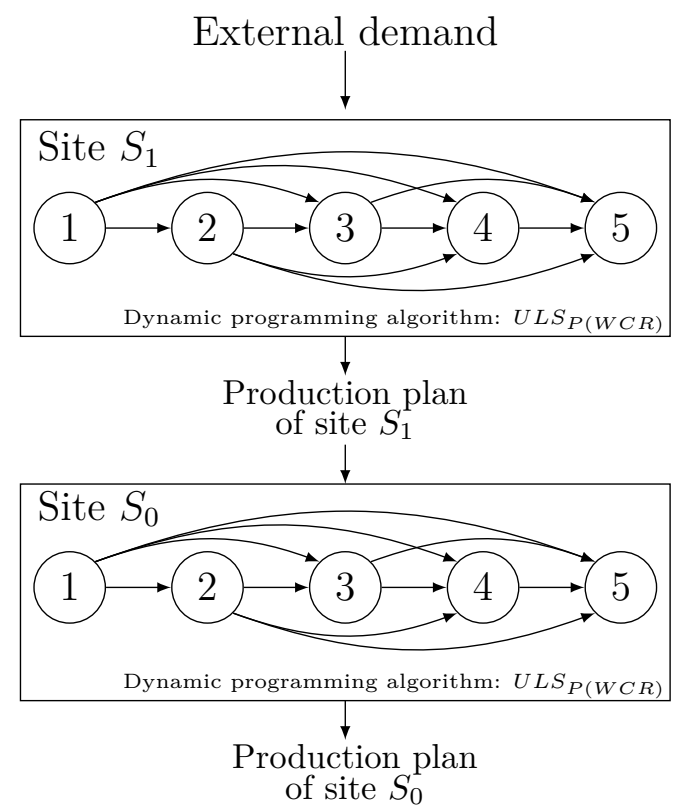

Figure 5.3 - Sequential approach

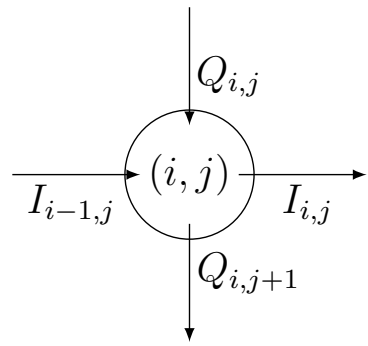

Figure 5.4 - A node and associated arcs in the Zangwill's network presentation of the multi-level problem 
$I_{i-1, j}$. Consequently, the associated WCR can not be correctly measured. For this reason, Zangwill's algorithm can not be directly applied for this problem. As a consequence, a new concept is required to tackle this problem. To do so, we first prove the ZIO property remains valid for this two-level problem. Then, the new concept will be explicitly described in the following. In this centralized problem, the delivery delay is not considered because it will significantly amplify the discount effect on the cost of the manufacturer when the delivery delay is theoretically great. It makes the NPV of the costs and the revenues of the manufacturer negligible and has no impact in the optimization of two-level plans. To avoid this unrealistic situation, the delivery is not considered in this approach.

Theorem 5.4.1. There exists a set of optimal production plans of the supplier and the manufacturer in which $I_{i, t-1} \times Q_{i t}=0$ for all $t$ and $i \in\{0,1\}$ (where $I_{i t}$ is the inventory of site $i$ at the end of the period $t)$ in $2 U L S_{P(B F R)}$ problem with associated assumptions in section 5.3.1.

\section{Proof of the ZIO property in centralized approach for $2 U L S_{P(W C R)}$ problem}

In the single-level case, the NPV of revenue is fixed by the deterministic demand that will not vary during the planning process. However, in two-level case, the revenue NPV of the supplier depends on the production program of the manufacturer. This program is not fixed a priori and is determined with a centralized optimization objective. Therefore, the revenue NPV of the supplier may vary according to the manufacturer's production plan and needs to be considered in the optimization procedure. However, the revenue NPV of the manufacturer is still fixed by the known external demands which do not vary in the planning process. It is omitted in the following proof.

The basic idea of this proof is similar to the previous one for single-level problem. The details of the proof are described as follows:

Firstly, we denote that :

- $X^{\left(2 U L S_{P(W C R)}\right)}$ represents all feasible solutions fro the two sites;

- $P$ denotes a set of feasible production plans of both sites;

- Profit $(P)$ stands for the objective value in the proposed model with the set of plans $P$.

Definition 2. A production planning is complied with the ZIO property if and only if the production is only planned when the inventory level drops to zero. With the notation of the two-level problem, it can mathematically be expressed as

$$
Q_{i t} \times\left[\sum_{k=1}^{t-1}\left(\sum_{l=t}^{T} X_{i l k}\right)\right]=0 \quad \forall t \in[2, T], i \in\{0,1\}
$$

In other words, it can be written as :

$$
\left\{\begin{array}{lll}
Q_{i t}>0 & \Rightarrow & X_{i k l}=0 \\
X_{i k l}>0 & \Rightarrow & \forall(k, l) \in[1, t-1] \times[t, T] \\
\text { it }=0 & \forall t \in[k+1, l], i \in\{0,1\}
\end{array}\right.
$$

Then, we denote that there is the optimal plan set $P^{*} \in X^{\left(2 U L S_{P(W C R)}\right)}$, where

$$
\operatorname{Profit}\left(P^{*}\right)=\max _{P \in X^{\left(2 U L S_{P(B F R)}\right)}} \operatorname{Profit}(P)
$$

Let $P_{i}$ (with $i \in\{0,1\}$ ) be a feasible planning which does not comply with the ZIO property. Note that $(t, m, n) \in[1, T]^{3}$ is a triplet with $m<t \leq n$. Thus, a violation of the property can be presented as $Q_{t} \times X_{m, n}>0$. Then, we assume that at least one violation can be found in $P_{i}$.

We consider a plan $P_{i}^{\prime}$ as a plan derived from $P_{i}$ in the following way and the ZIO structure can thus be found in $P_{i}^{\prime}$ :

$$
\begin{aligned}
& X_{i k t}^{\prime}=X_{i k t} \quad \forall(k, t) \in[1, T]^{2}-\{(m, n),(t, n)\}, i \in\{0,1\} \\
& X_{i m n}^{\prime}=0 \\
& X_{i t n}^{\prime}=X_{i t n}+X_{i m n}
\end{aligned}
$$


The violation can be found in the planning of either level. Therefore, we examine the objective value variations in the two following cases. In each case, we have a feasible plan at one level and we eliminate a violation of the ZIO property at the other level:

- case 1: Compare $P=\left\{P_{0}, P_{1}^{\prime \prime}\right\}$ with $P^{\prime}=\left\{P_{0}^{\prime}, P_{1}^{\prime \prime}\right\}$, where $P_{1}^{\prime \prime}$ is any feasible plan of the manufacturer;

- case 2: Compare $P=\left\{P_{0}^{\prime \prime}, P_{1}\right\}$ with $P^{\prime}=\left\{P_{0}^{\prime \prime}, P_{1}^{\prime}\right\}$, where $P_{0}^{\prime \prime}$ is any feasible plan of the supplier;

To simplify the NPV formulation, denote that $a_{i t}^{j}=a_{i} \frac{1}{\left(1+\alpha_{j}\right)^{t}}, s_{i t}^{j}=s_{i} \frac{1}{\left(1+\alpha_{j}\right)^{t}}, p_{i t}^{j}=$ $p_{i} \frac{1}{\left(1+\alpha_{j}\right)^{t}}, h_{i t}^{j}=h_{i} \frac{1}{\left(1+\alpha_{j}\right)^{t}}$ and $v_{i t}^{j}=v_{i} \frac{1}{\left(1+\alpha_{j}\right)^{t}}$.

\section{Case 1 :}

In this case, we change one period of the supplier's planning into ZIO structure. The change at supplier level will not impact the profit of the manufacturer. For this reason, this case can be considered as a single-level problem. According to the ZIO property in the single-level problem, changing into $P_{0}^{\prime}$ decreases the total cost and increases the profit of supplier. In other word, eliminating ZIO violation globally improves the profit of the chain in this case.

\section{Case 2 :}

In case 2, we move backward the production of $X_{1 m n}$ in $S_{1}$ from the period $m$ to the period $t$ in order to ensure a ZIO structure in the period $t$ at manufacturer level. It delays the delivery from $S_{0}$ which increases the NPV of the inventory holding cost and of the financial costs of supplier. The latter is due to the delayed payments of manufacturer. However, it will simultaneously decrease the NPV of all logistic and financial costs of the manufacturer. All NPV variations due to this transformation are summarized in Table 5.5 .

\begin{tabular}{|c|c|c|c|c|}
\hline & \multicolumn{4}{|c|}{ Variation of revenue } \\
\hline $\begin{array}{c}\text { Supplier } \\
\text { Manufacturer }\end{array}$ & \multicolumn{4}{|c|}{$\searrow$} \\
\hline \hline Variation of LC & Purchasing & Setup & Production & Holding \\
\hline Supplier & $=$ & $=$ & $=$ & $\nearrow$ \\
Manufacturer & $\searrow$ & $\searrow$ or $=$ & $\searrow$ & $\searrow$ \\
\hline \hline Variation of WCR & $W C R^{\text {pur }}$ & $W C R^{\text {set }}$ & $W C R^{\text {prod }}$ & $W C R^{\text {inv }}$ \\
\hline Supplier & $\nearrow$ & $\nearrow$ & $\nearrow$ & $\nearrow$ \\
Manufacturer & $\searrow$ & $\searrow$ & $\searrow$ & $\searrow$ \\
\hline
\end{tabular}

Table 5.5 - Variations due to the transformation for moving $X_{1 m n}$

Thus, we examine the objective value and express the mathematical formulation of these variations at two levels.

Firstly, at supplier level, we calculate the difference in logistic costs, $L C_{0}$. Obviously, since the planning of $S_{0}$ remains the same, the only difference can be found in inventory holding cost.

$$
\Delta L C_{0}=L C_{0}\left(P_{0}, P_{1}\right)-L C_{0}\left(P_{0}, P_{1}^{\prime}\right)=\Delta L C_{0}^{i n v}=-X_{1 m n} \sum_{j=m}^{t-1} h_{0 j}^{0}<0
$$

Next, we examine the variation of the financial cost through all its components. All of them 
increase because the delayed delivery of $X_{1 m n}$ extends the time length of financing these expenses.

$$
\begin{aligned}
& \Delta W C R_{0}^{\text {pur }}=W C R_{0}^{\text {pur }}\left(P_{0}, P_{1}\right)-W C R_{0}^{\text {pur }}\left(P_{0}, P_{1}^{\prime}\right) \\
& =\sum_{t=1}^{T} \sum_{k=t}^{T} a_{0} X_{0 t k}\left[\sum_{j=t+r_{0}}^{T} \frac{1}{\left(1+\alpha_{0}\right)^{j}}-\sum_{j=k+r_{1}}^{T} \frac{1}{\left(1+\alpha_{0}\right)^{j}}\right] \\
& -\sum_{t=1}^{T} \sum_{k=t}^{T} a_{0} X_{0 t k}^{\prime}\left[\sum_{j=t+r_{0}}^{T} \frac{1}{\left(1+\alpha_{0}\right)^{j}}-\sum_{j=k+r_{1}}^{T} \frac{1}{\left(1+\alpha_{0}\right)^{j}}\right] \\
& =-X_{1 m n}\left[\sum_{j=m}^{T} a_{0 j}^{0}-\sum_{j=t}^{T} a_{0 j}^{0}\right]=-X_{1 m n} \sum_{j=m}^{t-1} a_{0 j}^{0}<0 \\
& \Delta W C R_{0}^{\text {prod }}=W C R_{0}^{\text {prod }}\left(P_{0}, P_{1}\right)-W C R_{0}^{\text {prod }}\left(P_{0}, P_{1}^{\prime}\right) \\
& =\sum_{t=1}^{T} \sum_{k=t}^{T} p_{0} X_{0 t k} \sum_{j=t}^{k+r_{1}-1} \frac{1}{(1+\alpha)^{t}}-\sum_{t=1}^{T} \sum_{k=t}^{T} p_{0} X_{0 t k}^{\prime} \sum_{j=t}^{k+r_{1}-1} \frac{1}{(1+\alpha)^{t}} \\
& =-X_{1 m n}\left[\sum_{j=m}^{k+r_{1}-1} p_{j}^{0}-\sum_{j=t}^{k+r_{1}-1} p_{j}^{0}\right]=-X_{1 m n} \sum_{j=m}^{t-1} p_{0 j}^{0}<0 \\
& \begin{aligned}
\Delta W C R_{0}^{i n v} & =W C R_{0}^{i n v}\left(P_{0}, P_{1}\right)-W C R_{0}^{i n v}\left(P_{0}, P_{1}^{\prime}\right) \\
& =\sum_{t=1}^{T} \sum_{k=t}^{T} h_{0} X_{0 t k} \sum_{l=t}^{k-1} \sum_{j=l}^{k+r_{1}-1} \frac{1}{(1+\alpha)^{t}}-\sum_{t=1}^{T} \sum_{k=t}^{T} h_{0} X_{0 t k}^{\prime} \sum_{l=t}^{k-1} \sum_{j=l}^{k+r_{1}-1} \frac{1}{(1+\alpha)^{t}} \\
& =X_{1 m n}\left(\sum_{l=0}^{n-t-1} \sum_{k=m}^{t-1} h_{0, k+l}^{0}+\sum_{l=m+n-t}^{n-1} \sum_{j=l}^{n+r_{2}-1} h_{0 j}^{0}\right) \\
& =-X_{1 m n}\left(\sum_{l=0}^{n-t-1} \sum_{k=m}^{t-1} h_{0, k+l}^{0}+\sum_{l=m+n-t}^{n-1} \sum_{j=l}^{n+r_{1}-1} h_{0 j}^{0}\right)<0
\end{aligned} \\
& \Delta W C R_{0}^{\text {set }}=W C R_{0}^{\text {set }}\left(P_{0}, P_{1}\right)-W C R_{0}^{\text {set }}\left(P_{0}, P_{1}^{\prime}\right) \\
& =\sum_{t=1}^{T} \sum_{k=t}^{T}\left(\frac{s_{0 t} Y_{0 t} X_{0 t k}}{Q_{0 t}+\left(1-Y_{0 t}\right)} \sum_{j=t}^{k+r_{1}-1} \frac{1}{(1+\alpha)^{t}}\right) \\
& -\sum_{t=1}^{T} \sum_{k=t}^{T}\left(\frac{s_{0 t} Y_{0 t} X_{0 t k}^{\prime}}{Q_{0 t}^{\prime}+\left(1-Y_{0 t}\right)} \sum_{j=t}^{k+r_{1}-1} \frac{1}{(1+\alpha)^{t}}\right) \\
& =-\frac{X_{1 m n}}{Q_{0 m}} \sum_{j=m}^{t-1} s_{0 j}^{0}<0
\end{aligned}
$$

The NPV changes of the supplier's revenue can be expressed as $\Delta R_{0}=X_{1 m n}\left(v_{0, m+r_{1}}^{0}-v_{0, t++r_{1}}^{0}\right)>0$ because the $X_{1 m n}$ will be paid in a later period due to this transformation and the payment is further discounted over time.

Secondly, we calculate the differences at manufacturer level : By transforming from $P_{1}$ to $P_{1}^{\prime}$, the 
production of $X_{1 m n}$ is delayed from the period $m$ to $t$. The difference of the logistic cost is thus :

$$
\begin{aligned}
\Delta L C_{1} & =L C_{1}\left(P_{0}, P_{1}\right)-L C_{1}\left(P_{0}, P_{1}^{\prime}\right) \\
& =X_{1 m n}\left[\left(a_{1, m+r_{1}}^{1}-a_{1, t+r_{1}}^{1}\right)+\left(p_{1 m}^{1}-p_{1 t}^{1}\right)+\sum_{j=m}^{t-1} h_{1 j}^{1}\right]+\left(s_{m}^{1} Y_{1 m}-s_{m}^{1} Y_{1 m}^{\prime}\right)
\end{aligned}
$$

We need to separate the purchasing cost and inventory holding cost from the others. Thus, the variation of

- the purchasing cost of the manufacturer: $\Delta L C_{1}^{\text {pur }}=X_{1 m n}\left(a_{1, m+r_{1}}^{1}-a_{1, t+r_{1}}^{1}\right)>0$

- the inventory holding cost of the manufacturer: $\Delta L C_{1}^{i n v}=X_{1 m n} \sum_{j=m}^{t-1} h_{1 j}^{1}>0$

- the setup and production of the manufacturer:

$$
\begin{gathered}
X_{1 m n}\left(p_{1 m}^{1}-p_{1 t}^{1}\right)+\left(s_{m}^{1} Y_{1 m}-s_{m}^{1} Y_{1 m}^{\prime}\right) \\
>\quad 0 \quad \text { with } Y_{1 m} \geq Y_{1 m}^{\prime} \text { and } Y_{1 t}=Y_{1 t}^{\prime}=1
\end{gathered}
$$

For the financial cost, the relation of $W C R_{1}(P)>W C R_{1}\left(P^{\prime}\right)$ has been proved in the single-level case. However, we need to evaluate the difference on the purchasing WCR cost which is in a form similar to the ones at supplier level:

$$
\begin{aligned}
\Delta W C R_{1}^{\text {pur }}= & W C R_{1}^{\text {pur }}\left(P_{0}, P_{1}\right)-W C R_{1}^{\text {pur }}\left(P_{0}, P_{1}^{\prime}\right) \\
= & \sum_{t=1}^{T} \sum_{k=t}^{T} a_{1} X_{1 t k}\left[\sum_{j=t+r_{1}}^{T} \frac{1}{(1+\alpha)^{j}}-\sum_{j=k+r_{2}}^{T} \frac{1}{(1+\alpha)^{j}}\right] \\
& -\sum_{t=1}^{T} \sum_{k=t}^{T} a_{1} X_{1 t k}^{\prime}\left[\sum_{j=t+r_{1}}^{T} \frac{1}{(1+\alpha)^{j}}-\sum_{j=k+r_{2}}^{T} \frac{1}{(1+\alpha)^{j}}\right] \\
= & X_{1 m n}\left[\sum_{j=m}^{T} a_{1 j}^{1}-\sum_{j=t}^{T} a_{1 j}^{1}\right]=X_{1 m n} \sum_{j=m}^{t-1} a_{1 j}^{1}>0 \\
\Delta W C R_{1}^{\text {prod }}= & X_{1 m n} \sum_{j=m}^{t-1} p_{1 j}^{1}>0 \\
\Delta W C R_{1}^{\text {inv }}= & X_{1 m n}\left(\sum_{l=0}^{n-t-1} \sum_{k=m}^{t-1} h_{1, k+l}^{1}+\sum_{l=m+n-t}^{n-1} \sum_{j=l}^{n+r_{2}-1} h_{1 j}^{1}\right)>0
\end{aligned}
$$

The change in WCR for the setup cost at the manufacturer level is positive as proven in the singlelevel case:

$$
\begin{aligned}
\Delta W C R_{1}^{s e t}= & \sum_{t=1}^{T} \sum_{k=t}^{T}\left(\frac{s Y_{1 t} X_{1 t k}}{X_{1 t}+\left(1-Y_{1 t}\right)} \sum_{j=t}^{k+r_{2}-1} \frac{1}{(1+\alpha)^{t}}\right) \\
& -\sum_{t=1}^{T} \sum_{k=t}^{T}\left(\frac{s Y_{1 t} X_{1 t k}^{\prime}}{X_{1 t}^{\prime}+\left(1-Y_{1 t}\right)} \sum_{j=t}^{k+r_{2}-1} \frac{1}{(1+\alpha)^{t}}\right)>0
\end{aligned}
$$

Once expressed all variations of NPVs of the related revenue and costs, we combine them to calculate the global difference due to this transformation in following order: 
1. The variation of revenue NPV of the supplier minus the variation of purchasing cost NPV of the manufacturer;

2. The sum of the NPV variations of inventory holding costs of both levels;

3. The sum of the WCR variations of purchasing cost of the manufacturer and all WCR of the supplier excluding the WCR of inventory holding cost;

4. The sum of the WCR variations of inventory holding cost of both levels.

First, the variation of purchasing cost NPV of the manufacturer minus the variation of revenue NPV of the supplier is calculated as below:

$$
\Delta L C_{1}^{\text {pur }}-\Delta R_{0}=X_{1 m n}\left(a_{1, m+r_{1}}^{1}-a_{1, t+r_{1}}^{1}\right)-X_{1 m n}\left(v_{0, m+r_{1}}^{0}-v_{0, t+r_{1}}^{0}\right)
$$

As assumed, $\alpha_{1}<\alpha_{0}$, we thus have $\frac{1}{1+\alpha_{1}}>\frac{1}{1+\alpha_{0}}$. Since $v_{0}=a_{1}, a_{1, i}^{1}=a_{1} \frac{1}{\left(1+\alpha_{1}\right)^{i}}=$ $v_{0} \frac{1}{\left(1+\alpha_{1}\right)^{i}}>v_{0} \frac{1}{\left(1+\alpha_{0}\right)^{i}}=v_{0, i}^{0}$. Therefore, $\Delta R_{0}-\operatorname{Pur}_{1}<0$

Second, since we assume $h_{0}<h_{1}$, the sum of the NPV variations of inventory holding costs of both levels is positive:

$$
\begin{gathered}
\Delta L C_{0}^{i n v}+\Delta L C_{1}^{i n v} \\
=\quad X_{1 m n} \sum_{j=m}^{t-1}\left(h_{1 j}^{1}-h_{0 j}^{0}\right) \\
>\quad X_{1 m n} \sum_{j=m}^{t-1}\left(h_{1 j}^{0}-h_{0 j}^{0}\right) \\
>\quad X_{1 m n} \sum_{j=m}^{t-1}\left(h_{0 j}^{0}-h_{0 j}^{0}\right)=0
\end{gathered}
$$

Third, in practice, the unit sales price of supplier (i.e. the unit purchasing cost of manufacturer) is usually greater than average cost per intermediate product including all logistic and financial cost in order to gain profit for the supplier site. Therefore, we adopt a simplified formulation to express the relation between $a_{1}$ and all cost parameters of the supplier that $a_{1} \geq a_{0}+\overline{s_{0}}+p_{0}+h_{0}$ (where, $\overline{s_{0}}=\max _{t \in T \text { and } Q_{0 t} \neq 0}\left\{\frac{s_{0}}{Q_{0 t}}\right\}$ ). For this reason, we could obtain that

$$
\begin{gathered}
\Delta W C R_{1}^{\text {pur }}+\Delta W C R_{0}^{\text {pur }}+\Delta W C R_{0}^{\text {prod }}+\Delta W C R_{0}^{\text {set }} \\
=\quad X_{1 m n} \sum_{j=m}^{t-1} a_{1 j}^{1}-X_{1 m n} \sum_{j=m}^{t-1} a_{0 j}^{0}-X_{1 m n} \sum_{j=m}^{t-1} p_{0 j}^{0}-\frac{X_{1 m n}}{Q_{0 m}} \sum_{j=m}^{t-1} s_{0 j}^{0} \\
>\quad X_{1 m n} \sum_{j=m}^{t-1} a_{1 j}^{0}-X_{1 m n} \sum_{j=m}^{t-1} a_{0 j}^{0}-X_{1 m n} \sum_{j=m}^{t-1} p_{0 j}^{0}-\frac{X_{1 m n}}{Q_{0 m}} \sum_{j=m}^{t-1} s_{0 j}^{0} \\
>\quad X_{1 m n} \sum_{j=m}^{t-1}\left(a_{0}^{0}+\overline{s_{0}^{0}}+p_{0}^{0}\right)-X_{1 m n} \sum_{j=m}^{t-1} a_{0 j}^{0}-X_{1 m n} \sum_{j=m}^{t-1} p_{0 j}^{0}-\frac{X_{1 m n}}{Q_{0 m}} \sum_{j=m}^{t-1} s_{0 j}^{0}>0
\end{gathered}
$$

Last, we assume the $r_{1} \geq r_{2}$. In addition, we have $h_{0}<h_{1}$ and $\alpha_{1}<\alpha_{0}$. Consequently, $h_{1, i}^{1}>h_{0, i}^{0}$. Therefore, 


$$
\begin{gathered}
\Delta W C R_{0}^{i n v}+\Delta W C R_{1}^{i n v} \\
-X_{1 m n}\left(\sum_{1 m n}\left(\sum_{l=0}^{n-t-1} \sum_{k=m}^{t-1} h_{1, k+l}^{1}+\sum_{l=m+n-t}^{n-t-1} \sum_{j=l}^{n-1} \sum_{k=m}^{t-1} h_{0, k+l}^{0}+\sum_{l=m+n-t}^{n-1} \sum_{j=l}^{n+r_{1}-1} h_{0 j}^{0}\right)>0\right.
\end{gathered}
$$

In summary, combining the equation (5.22), (5.25), (5.26), (5.27) and (5.28), the sum of total logistic and financial cost minus the difference in revenue NPV of the supplier is positive. This means that the total cost will be reduced by changing $P_{1}$ to $P_{1}^{\prime}$. Thus, we favor the ZIO type planning which improves the global profit.

To conclude, as proven above, the ZIO property remains valid in this two-level problem. It allows us to established a dynamic programming-based algorithm considering the financial cost of the WCR and the interdependency between levels.

\section{Description of the centralized approach}

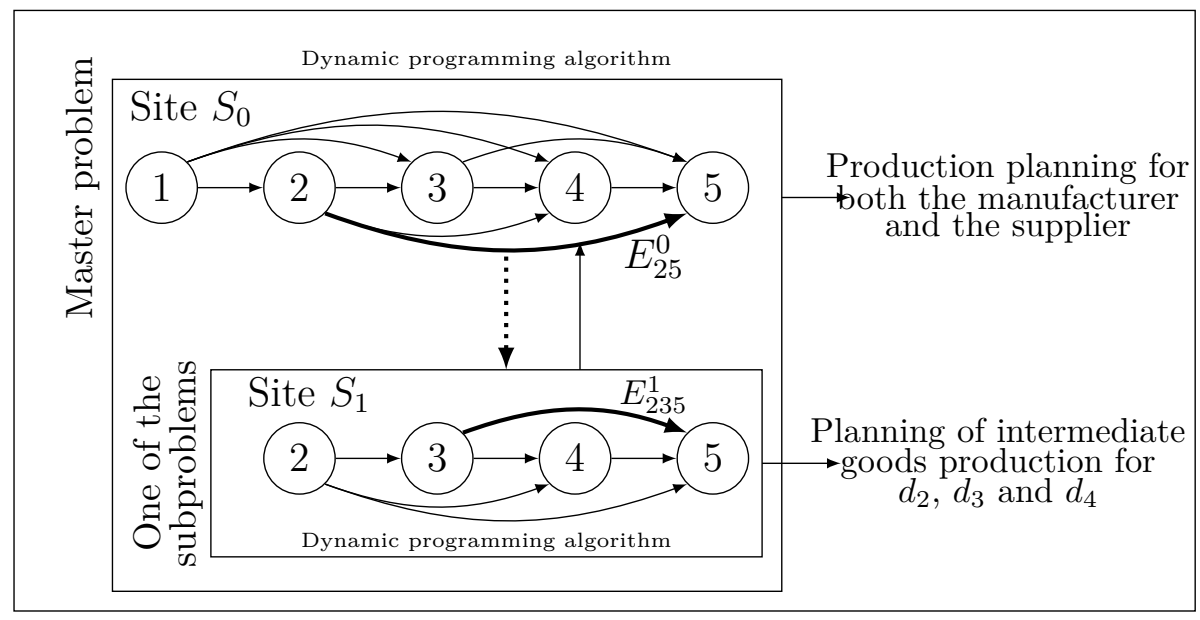

Figure 5.5 - Centralized approach for $2 U L S_{P(W C R)}$

For this centralized approach, we propose a reformulation in the form of two-level shortest paths which represents the two-level supply chain as shown in Figure 5.5. In the reformulation, for both levels, the nodes represent the planning periods with a dummy period at the end. As the ZIO property have been proven valid and the one-to-one production assumption, passing an arc, $E$, from the period $t$ to $k$ represents a production of corresponding products (semi-finished at the supplier level or finished at the manufacturer level) for all external demands between periods $t$ and $k-1$ at the period $t$ in site $S_{i}$ with $i \in\{0,1\}$. We firstly consider a master problem to determine the shortest path at the supplier level. In order to take into account the interdependency between these two levels, the value of the arc at the supplier level includes not only the NPV of the revenue and all logistic and financial cost of the supplier, but also the NPV of all costs of the manufacturer. To calculate these costs and revenue associated to an $\operatorname{arc} E_{t k}^{0}$ at supplier level from $t$ to $k$,

- on the one hand, we first calculate the discounted costs of the supplier for such a production that do not depend on the manufacturer's plan. To do so, since we have all intermediate products made in the period $t$ at the supplier level, we can directly compute the discounted costs of the supplier including:

— purchasing cost; 
- setup cost;

- production cost.

- on the other hand, we should also add all the costs and the revenue that depend on the manufacturer's plan that includes the NPV of

— the inventory holding cost of the supplier;

- the WCR financial cost of the supplier;

- the revenue of the supplier;

- all logistic and financial costs of the manufacturer between period $t$ to $k$.

These terms are computed by solving a sub-problem which determines the optimal plan of the manufacturer that minimizes the sum of these costs minus the revenue of the supplier, for satisfying the external demands from $t$ to $k-1$, denoted as $d_{t k}=\sum_{i=t}^{k-1} d_{i}$.

For the $E_{t k}^{0}$ of the master problem and the associated sub-problem, the productions at both levels are only organized for satisfying $d_{t k}$ in order to comply with the ZIO property at both levels. It means that, firstly, the intermediate product for $d_{j}$ with $j>k$ would not be produced between the periods $t$ and $k-1$ in the supplier because there is another production planned in the period $k$; secondly, the final product for $d_{j}$ with $j>k$ would not be produced between the period $t$ and $k-1$ because the corresponding intermediate product have not be prepared at the supplier level during these periods.

Therefore, in the sub-problem of $E_{t k}^{0}$, the arc $E_{t t^{\prime} k^{\prime}}^{1}$, with $t \leq t^{\prime}, k^{\prime} \leq k$, of the sub-problem at manufacturer level represents a production for $d_{t^{\prime} k^{\prime}}$ at the period $t^{\prime}$. For this production, all intermediate products have been manufactured at the period $t$ by the supplier and delivered to the manufacturer at the period $t^{\prime}$. Therefore, we are able to calculate the value of the arc $E_{t t^{\prime} k^{\prime}}^{1}$ that consists of:

- the inventory holding cost of the supplier for holding the intermediate products for the external demands between the period $t^{\prime}$ and $k^{\prime}-1$ (denoted as $d_{t^{\prime} k^{\prime}}$ ) during the periods from $t$ to $t^{\prime}-1$;

- the WCR financial cost to cover all operations costs associated to the intermediate goods production for $d_{t k}$ of the supplier until the correspondent payment arrived from the manufacturer in the period $t^{\prime}+r_{1}$;

- the revenue of the supplier of selling the intermediate goods for $d_{t^{\prime} k^{\prime}}$ that will be received in the period $t^{\prime}+r_{1}$.

- All logistic and financial costs for a production of $d_{t^{\prime} k^{\prime}}$ at the period $t^{\prime}$ by the manufacturer. The manufacturer would receive the payment from the external customer according to its demand and the payment delay, $r_{2}$.

After formulating the problem in the form of a two-level shortest paths, both the master problem and sub-problem can be solved by a dynamic programming algorithm similar to the single-level one (whose complexity is $O\left(T^{4}\right)$ ). Since there are $O\left(T^{2}\right)$ sub-problems to be dealt with and each one is computed at worst in $O\left(T^{4}\right)$, the complexity of this centralized approach is thus $O\left(T^{6}\right)$. The detail of the algorithm is presented in the following section. The optimality of this approach is ensured by the fact that this approach enumerates all possible combinations of production plans of both levels complying with the ZIO property.

As we state in the introduction of the decentralized approach, the application of the centralized approach may not be applicable in a supply chain with independent companies. However, in the context of the RCSM project, the optimization platform provides a global management for all members of the supply chain and financial solutions for cover their WCRs which are gathered in a WCR pooling. It is still reasonable to employ the centralized approach. Furthermore, we assume that these members benefit from a same interest rate for financing the pooled WCR. 


\section{Algorithm of centralized approach}

As presented in Figure 5.5, this approach has a Russian doll type procedure. This procedure consists in dynamically calculating the optimal (sub)plan of the manufacturer every time we compute the arc value for the master problem at the supplier level in the recursion. Therefore, key elements are the formulations of the arc value of the sub-problem and of the master problem. The former allows us to integrate the interdependency between levels by including all costs and revenues that depend on the manufacturer's plan. Once these arc values are calculated, we are able to directly apply the recursion of the single-level algorithm to obtain the optimal value of the subproblem. The latter differs from the ones in the single-level problem by taking into account the optimal value of the corresponding subproblem. Let denote that

- We denote a sub-problem, associated to the $E_{t k}^{0}$ of the master problem, is $S u b_{t k}$;

- The arc value of the master problem between period $t$ and $k$, is $E_{t k}^{0}$;

- For valuing $E_{t k}^{0}$, we need to optimally resolve the $S u b_{t k}$. The arc value between $t^{\prime}$ and $k^{\prime}$ in $S u b_{t k}$ at level 1 is denoted $E_{t k t^{\prime} k^{\prime}}^{1}$.

- The optimal value to reach the node $k^{\prime}$ at level 1 from node $t$ in $S u b_{t k}$ is denoted as $O p t_{t k^{\prime}}^{1}$. We know that all required intermediate goods are delivered at period $t$ by the supplier.

First, in $S u b_{t k}$, the arc value of $E_{t k t^{\prime} k^{\prime}}^{1}$ consists of the inventory holding costs (denoted $I n v_{t t^{\prime} k^{\prime}}^{0}$ ), all WCR financing costs $\left(W C R_{t k t^{\prime} k^{\prime}}^{0}\right)$ and the revenue $\left(R_{t^{\prime} k^{\prime}}^{0}\right)$ of the supplier as well as all logistic and financial cost of the manufacturer for a production of $d_{t^{\prime} k^{\prime}}$ in the period $t^{\prime}$. The four index of $W C R_{t k t^{\prime} k^{\prime}}^{0}$ are all necessary. $W C R_{t k t^{\prime} k^{\prime}}^{0}$ presents the WCR of the supplier logistic cost that depends on the production of $d_{t^{\prime} k^{\prime}}$. In addition, $t$ and $k$ are used to link this calculation to the associated sub-problem $S u b_{t k}$. Especially, all products manufactured at the period $t$ by the supplier uniformly share the setup cost. With this unit setup cost, the WCR of setup cost is computed. The quantity of these products is found as $d_{t k}$ is the equation 5.30 . Since we calculate $E_{t k t^{\prime} k^{\prime}}^{1}$, the intermediate goods to satisfy the external demand from $d_{t^{\prime}}$ to $d_{k^{\prime}-1}$ are already produced by the supplier at the period $t$ and will be delivered to the manufacturer at period $t^{\prime}$. Furthermore, according to the ZIO property proven for the $2 U L S_{P(W C R)}$ problem, the production quantity can only be the sum of demands in the following periods. Thus, the components of $E_{t k t^{\prime} k^{\prime}}^{1}$ can be respectively written as:

$$
\begin{gathered}
\operatorname{Inv}_{t t^{\prime} k^{\prime}}^{0}=\sum_{l=t}^{t^{\prime}-1} \sum_{q=t^{\prime}}^{k^{\prime}-1} h_{0 l}^{0} d_{q} \\
W C R_{t k t^{\prime} k^{\prime}}^{0}=a_{0} \cdot \sum_{q=t^{\prime}}^{k^{\prime}-1} d_{q} \cdot\left(\sum_{j=t+r_{0}}^{T} \frac{1}{\left(1+\alpha_{0}\right)^{j}}-\sum_{j=t^{\prime}+r_{1}}^{T} \frac{1}{\left(1+\alpha_{0}\right)^{j}}\right) \\
+p_{0} \cdot \sum_{q=t^{\prime}}^{k^{\prime}-1} d_{q} \cdot \sum_{j=t}^{t^{\prime}+r_{1}-1} \frac{1}{\left(1+\alpha_{0}\right)^{j}} \\
+\frac{s_{0}}{d_{t k}} \cdot \sum_{q=t^{\prime}}^{k^{\prime}-1} d_{q} \cdot \sum_{j=t}^{t^{\prime}+r_{1}-1} \frac{1}{\left(1+\alpha_{0}\right)^{j}} \\
+h_{0} \cdot \sum_{q=t^{\prime}}^{k^{\prime}-1} d_{q} \cdot \sum_{w=t}^{t^{\prime}-1} \sum_{j=w}^{t^{\prime}+r_{1}-1} \frac{1}{\left(1+\alpha_{0}\right)^{j}} \\
R_{t^{\prime} k^{\prime}}^{0}=v_{0, t^{\prime}+r_{1}}^{0} \sum_{l=t^{\prime}}^{k^{\prime}-1} d_{l}
\end{gathered}
$$

The revenue, $R_{t^{\prime} k^{\prime}}^{0}$, only depends the manufacturer's production plan which is to produce the $d_{t^{\prime} k^{\prime}}$ in the period $t^{\prime}$. Thus, the supplier would receive the payment in the period $t^{\prime}+r_{1}$. Moreover, the two 
other components, the logistic cost and the financial cost of the manufacturer, $L C_{t^{\prime} k^{\prime}}^{1}$ and $\beta_{1} W C R_{t^{\prime} k^{\prime}}^{1}$ are directly modeled with the proposed WCR model in section 5.3.2. Remind that, for instance, $a_{i t}^{j}=a_{i} \cdot \frac{1}{\left(1+\alpha_{j}\right)^{t}}$.

$$
\begin{gathered}
L C_{t^{\prime} k^{\prime}}^{1}=\left(a_{1, t^{\prime}+r_{1}}^{1}+p_{1 t^{\prime}}^{1}\right) \sum_{l=t^{\prime}}^{k^{\prime}-1} d_{l}+s_{1 t^{\prime}}^{1}+\sum_{l=t^{\prime}}^{k^{\prime}-1} d_{l} \sum_{q=t^{\prime}}^{l-1} h_{1 q}^{1} \\
W C R_{t^{\prime} k^{\prime}}^{1}=\sum_{l=t^{\prime}}^{k^{\prime}-1} d_{l}\left[\left(\sum_{j=t^{\prime}}^{T} a_{1, j+r_{1}}^{1}-\sum_{j=l}^{T} a_{1, j+r_{2}}^{1}\right)+\sum_{j=t^{\prime}}^{l+r_{2}-1} p_{1 j}^{1}+\sum_{j=t^{\prime}}^{l+r_{2}-1} \frac{s_{1 j}^{1}}{d_{t^{\prime} k^{\prime}}}+\sum_{q=t^{\prime}}^{l-1} \sum_{j=q}^{l+r_{2}-1} h_{1 j}^{1}\right]
\end{gathered}
$$

Combining the holding cost and the financial cost of the supplier and all costs of the manufacturer, the formulation of $E_{t t^{\prime} k^{\prime}}^{1}$ is written as below :

$$
E_{t k t^{\prime} k^{\prime}}^{1}=\operatorname{Inv}_{t t^{\prime} k^{\prime}}^{0}+\beta_{0} W C R_{t k t^{\prime} k^{\prime}}^{0}-R_{t^{\prime} k^{\prime}}^{0}+L C_{t^{\prime} k^{\prime}}^{1}+\beta_{1} W C R_{t^{\prime} k^{\prime}}^{1}
$$

Using this formulation, we can compute the optimal value of this subproblem, $O p t_{t k k^{\prime}}^{1}$, by adopting a similar recursion than the one in the single-level algorithm which is :

$$
O p t_{t k k^{\prime}}^{1}=\min _{t^{\prime} \in\left[t, k^{\prime}-1\right]}\left\{O p t_{t k t^{\prime}}^{1}+E_{t k t^{\prime} k^{\prime}}^{1}\right\}
$$

Values of $O p t_{t k t}^{1}$ are set to zero. With this recursion, we sequentially compute the optimal value of $O p t_{t, k, t+1}^{1}$, then $O p t_{t, k, t+2}^{1}, \ldots$, until $O p t_{t, k, k}^{1}$. $O p t_{t k k}^{1}$ is the optimal value of the $S u b_{t k}$ which is needed to determine the value of $E_{t k}^{0}$ in the master problem.

Second, in the recursion of the master problem, we calculate the arc values at the supplier level. $E_{t k}^{0}$ thus includes the costs that are independent of the optimal plan fo the corresponding sub-problem (purchasing, setup and production costs) and the optimal value of the corresponding sub-problem. Therefore, the arc value at supplier level is written as:

$$
E_{t k}^{0}=\left(a_{0, t+r_{f}}^{\prime}+p_{0 t}^{\prime}\right) \sum_{l=t}^{k-1} d_{l}+s_{0 t}^{\prime}+O p t_{t k k}^{1}
$$

With all up-mentioned elements, the final recursion is formulated as below, where Cost $_{t}$ represents the minimal total cost of both sites minus the revenue of the supplier. Moreover, the beginning of the planning horizon is set to zero. Thus, Cost $_{0}=0$.

$$
\text { Cost }_{t}=\min _{j \in[0, t-1]}\left\{\operatorname{Cost}_{j}+E_{j t}^{0}\right\}
$$

To obtain the optimal value of the problem, we should sequentially compute Cost $_{t}$ from $t=1$ to $t=T$. The optimal value is Cost $_{T}$

The pseudo-code is given in Algorithm 2

\section{Illustrative example of arc value calculation and of the recursion}

The proposed arc valuation and the recursion are more intricate than in the single-level problem. Therefore, the associated calculation will be illustrated by solving a simple problem with a four periods horizon. The external demand and parameter values are provided in Table 5.6 et 5.7.

In this example, the calculations associated to the arc values of $E_{04}^{0}$ and $E_{0414}^{1}$ are gradually elaborated as well as the whole recursion. 




Table 5.6 - External demand of the illustrative example

\begin{tabular}{lllll}
\hline Period & 1 & 2 & 3 & 4 \\
\hline Demand & 10 & 20 & 15 & 30 \\
\hline
\end{tabular}

Table 5.7 - Parameter values of the illustrative example

\begin{tabular}{lll}
\hline Parameter & Supplier & Manufacturer \\
\hline Unit sales price & 15 & 25 \\
Purchasing unit cost, $a_{i}$ & 1 & 15 \\
Setup cost, $s_{i}$ & 60 & 75 \\
Production unit cost, $p_{i}$ & 2 & 1 \\
Inventory holding unit cost per period, $h_{i}$ & 1 & 3 \\
Discount rate, $\alpha$ & 0.03 & 0.01 \\
Interest rate, $\beta$ & 0.05 & 0.05 \\
Delays in payment & $r_{2}=r_{1}=r_{0}=1$ \\
\hline
\end{tabular}

In order to execute the recursion, all arc values at both levels are calculated and given in Table 5.8. These values are listed from the left to the right following the sequence of use in the recursion. The calculations are computed based on a planning horizon from period 0 to 3 and all results are rounded to two decimal places.

Firstly, according to the formulation presented in the previous section, $E_{0414}^{1}=\operatorname{Inv} v_{014}^{0}+\beta_{0} W C R_{0414}^{0}-$ $R_{14}^{0}+L C_{14}^{1}+\beta_{1} W C R_{14}^{1}$. For each term,

$$
\operatorname{Inv} v_{014}^{0}=\sum_{l=0}^{1-1} \sum_{q=1}^{4-1} h_{0 l}^{0} d_{q}=\left(\frac{1}{1+0.03}\right)^{0} \times 2 \times(20+15+30)=130
$$


Table 5.8 - Arc values of both levels

\begin{tabular}{|l|l|l|l|l|l|l|l|l|l|}
\hline$E_{01}^{0}$ & 185.84 & $E_{02}^{0}$ & 358.31 & $E_{03}^{0}$ & 550.41 & $E_{04}^{0}$ & $\mathbf{9 9 5 . 6 4}$ & & \\
\hline$E_{0101}^{1}$ & 96.13 & $E_{0201}^{1}$ & 94.13 & $E_{0301}^{1}$ & 93.80 & $E_{0401}^{1}$ & 93.53 & $E_{0423}^{1}$ & 179.03 \\
& & $E_{0202}^{1}$ & 209.19 & $E_{0302}^{1}$ & 208.19 & $E_{0402}^{1}$ & 207.39 & $E_{0404}^{1}$ & 780.30 \\
& & $E_{0212}^{1}$ & 162.87 & $E_{0312}^{1}$ & 161.55 & $E_{0412}^{1}$ & 160.50 & $E_{0414}^{1}$ & $\mathbf{6 5 7 . 3 5}$ \\
& & & & $E_{0303}^{1}$ & 356.72 & $E_{0403}^{1}$ & 355.51 & $E_{0424}^{1}$ & 505.43 \\
& & & & $E_{0313}^{1}$ & 286.57 & $E_{0413}^{1}$ & 284.73 & $E_{0434}^{1}$ & 360.31 \\
& & & & $E_{0323}^{1}$ & 180.20 & & & & \\
\hline \hline$E_{12}^{0}$ & 230.87 & $E_{13}^{0}$ & 361.99 & $E_{14}^{0}$ & 730.86 & $E_{23}^{0}$ & 207.92 & $E_{24}^{0}$ & 473.31 \\
\hline$E_{1212}^{1}$ & 114.93 & $E_{1312}^{1}$ & 113.68 & $E_{1412}^{1}$ & 112.91 & $E_{2323}^{1}$ & 109.36 & $E_{2423}^{1}$ & 107.47 \\
& & $E_{1313}^{1}$ & 202.79 & $E_{1413}^{1}$ & 201.44 & & & $E_{2424}^{1}$ & 290.74 \\
& & $E_{1323}^{1}$ & 143.15 & $E_{1423}^{1}$ & 142.01 & & & $E_{2434}^{1}$ & 212.42 \\
& & & & $E_{1414}^{1}$ & 502.67 & & & & \\
& & & & $E_{1424}^{1}$ & 394.37 & & & & \\
\hline \hline$E_{34}^{0}$ & 281.59 & & & & & & & & \\
\hline$E_{3434}^{1}$ & 145.12 & & & & & & & & \\
\hline
\end{tabular}

$$
\begin{aligned}
W C R_{0414}^{0} & =a_{0} \cdot \sum_{q=1}^{4-1} d_{q} \cdot\left(\sum_{j=0+r_{0}}^{T} \frac{1}{\left(0+\alpha_{0}\right)^{j}}-\sum_{j=1+r_{1}}^{T} \frac{1}{\left(0+\alpha_{0}\right)^{j}}\right) \\
& +p_{0} \cdot \sum_{q=1}^{4-1} d_{q} \cdot \sum_{j=1}^{1+r_{1}-1} \frac{1}{\left(1+\alpha_{0}\right)^{j}} \\
& +\frac{s_{0}}{d_{04}} \cdot \sum_{q=1}^{4-1} d_{q} \cdot \sum_{j=0}^{1+r_{1}-1} \frac{1}{\left(1+\alpha_{0}\right)^{j}} \\
& +h_{0} \cdot \sum_{q=1}^{4-1} d_{q} \cdot \sum_{w=0}^{1-1} \sum_{j=w}^{1+r_{1}-1} \frac{1}{\left(1+\alpha_{0}\right)^{j}} \\
& =\left(\frac{1}{1+0.03}\right)^{\times} \times 1 \times(20+15+30) \\
& +\left[\left(\frac{1}{1+0.03}\right)^{0}+\frac{1}{1+0.03}\right] \times 2 \times(20+15+30) \\
& +\left[\left(\frac{1}{1+0.03}\right)^{0}+\frac{1}{1+0.03}\right] \times \frac{60}{75} \times(20+15+30) \\
& +\left[\left(\frac{1}{1+0.03}\right)^{0}+\frac{1}{1+0.03}\right] \times 2 \times(20+15+30) \\
& =63.11+256.21+102.49+256.21 \\
& =678.02
\end{aligned}
$$

$$
R_{14}^{0}=v_{0,1+r_{1}}^{0} \sum_{l=1}^{4-1} d_{l}=\left(\frac{1}{1+0.03}\right)^{2} \times 15 \times(20+15+30)=919.03
$$




$$
\begin{aligned}
L C_{14}^{1} & =\left(a_{1,1+r_{1}}^{1}+p_{11}^{1}\right) \sum_{l=1}^{4-1} d_{l}+s_{11}^{1}+\sum_{l=1}^{4-1} d_{l} \sum_{q=1}^{l-1} h_{1 q}^{1} \\
& =\left(\frac{1}{1+0.01}\right)^{2} \times 15 \times(20+15+30)+\left(\frac{1}{1+0.01}\right) \times 1 \times(20+15+30) \\
& +\left(\frac{1}{1+0.01}\right) \times 75+3 \times\left[\left(\frac{1}{1+0.01}\right) \times(30+15)+\left(\frac{1}{1+0.01}\right)^{2} \times 30\right] \\
& =955.79+64.36+74.26+221.89 \\
& =1316.30
\end{aligned}
$$

$$
\begin{aligned}
W C R_{14}^{1}= & \sum_{l=1}^{4-1} d_{l}\left[\left(\sum_{j=1}^{T} a_{1, j+r_{1}}^{1}-\sum_{j=l}^{T} a_{1, j+r_{2}}^{1}+\sum_{j=1}^{l+r_{2}-1} p_{1 j}^{1}+\sum_{j=1}^{l+r_{2}-1} \frac{s_{1 j}^{1}}{d_{14}}+\sum_{q=1}^{l-1} \sum_{j=q}^{l+r_{2}-1} h_{1 j}^{1}\right]\right. \\
= & \left\{0+\left(\frac{1}{1+0.01}\right)^{2} \times 15 \times 15+\left[\left(\frac{1}{1+0.01}\right)^{2}+\left(\frac{1}{1+0.01}\right)^{3}\right] \times 15 \times 30\right\} \\
+ & \left\{\left(\frac{1}{1+0.01}\right) \times 1 \times 20+\left[\left(\frac{1}{1+0.01}\right)+\left(\frac{1}{1+0.01}\right)^{2}\right] \times 1 \times 15\right. \\
& \left.+\left[\left(\frac{1}{1+0.01}\right)+\left(\frac{1}{1+0.01}\right)^{2}+\left(\frac{1}{1+0.01}\right)^{3}\right] \times 1 \times 30\right\} \\
+ & \left\{\left(\frac{1}{1+0.01}\right) \times \frac{75}{65} \times 20+\left[\left(\frac{1}{1+0.01}\right)+\left(\frac{1}{1+0.01}\right)^{2}\right] \times \frac{75}{65} \times 15\right. \\
& \left.+\left[\left(\frac{1}{1+0.01}\right)^{3}+\left(\frac{1}{1+0.01}\right)^{2}+\left(\frac{1}{1+0.01}\right)^{3}\right] \times \frac{75}{65} \times 30\right\} \\
+ & \left\{\left[\left(\frac{1}{1+0.01}\right)^{1}+\left(\frac{1}{1+0.01}\right)^{2}\right] \times 3 \times 15\right. \\
& +\left[\left(\frac{1}{1+0.01}\right)^{1}+\left(\frac{1}{1+0.01}\right)^{2}+\left(\frac{1}{1+0.01}\right)^{3}\right] \times 3 \times 30 \\
= & 1098.47+137.58+158.76+528.94 \\
= & 1923.75 \times 3 \times 30\} \\
& {\left[\left(\frac{1}{1+0.01}\right)^{2}+\left(\frac{1}{1+0.01}\right)^{3}\right] \times 3 \times 30 }
\end{aligned}
$$

To sum up, $E_{0414}^{1}=130+0.05 \times 678.02-919.03+1316.3+0.05 \times 1923.75=657.35$.

Secondly, the value of $E_{04}^{0}$ consists of the fixed logistic cost of the supplier for a production of $d_{04}$ in the period 0 and $O p t_{044}^{1}$ calculated based on the optimal production plan of the manufacturer from period 0 to 3 . It is formulated as below: 


$$
\begin{aligned}
E_{04}^{0}= & \left(a_{0,0+r_{f}}^{\prime}+p_{0 t}^{\prime}\right) \sum_{l=0}^{4-1} d_{l}+s_{00}^{\prime}+O p t_{044}^{1} \\
= & {\left[\left(\frac{1}{1+0.03}\right)^{1}+\left(\frac{1}{1+0.03}\right)^{0}\right] \times 1 \times(10+20+15+30) } \\
& +\left(\frac{1}{1+0.03}\right)^{0} \times 60+O p t_{044}^{1} \\
= & 282.82+O p t_{044}^{1}
\end{aligned}
$$

In order to obtain the value of $O p t_{044}^{1}$, we should sequentially compute the $O p t_{04 k^{\prime}}^{1}$ from $k^{\prime}=1$ to $k^{\prime}=4$ using the recursion (5.4.3). These calculations are presented as follows:

$$
\begin{aligned}
& O p t_{041}^{1}=O p t_{040}^{1}+E_{0401}^{1}=\mathbf{0}+\mathbf{9 3 . 5 3}=93.53 \\
& O p t_{042}^{1}=\min \left\{O p t_{040}^{1}+E_{0402}^{1}, O p t_{041}^{1}+E_{0412}^{1}\right\} \\
& =\min \{\mathbf{0}+\mathbf{2 0 7 . 3 9}, 93.53+160.50\}=207.39 \\
& O p t_{043}^{1}=\min \left\{O p t_{040}^{1}+E_{0403}^{1}, O p t_{041}^{1}+E_{0413}^{1}, O p t_{042}^{1}+E_{0423}^{1}\right\} \\
& =\min \{\mathbf{0}+\mathbf{3 5 5 . 5 2}, 93.53+284.73,207.39+179.03\}=355.52 \\
& O p t_{044}^{1}=\min \left\{O p t_{040}^{1}+E_{0404}^{1}, O p t_{041}^{1}+E_{0414}^{1}, O p t_{042}^{1}+E_{0424}^{1}, O p t_{043}^{1}+E_{0434}^{1}\right\} \\
& =\min \{780.30,93.53+657.35, \mathbf{2 0 7 . 3 9}+\mathbf{5 0 5 . 4 3}, 355.52+360.31\}=712.82
\end{aligned}
$$

With this value, we obtain that $E_{04}^{0}=282.82+712.82=995.64$.

Once we obtain all values of $E_{t k}^{0}$ where $t \in\{0, T-1\}$ and $t<k \leq T$ as given in Table 5.8 , the optimal value of the problem Cost $_{T}$ can be calculated as follows:

$$
\begin{aligned}
\text { Cost }_{1} & =\text { Cost }_{0}+E_{01}^{0}=\mathbf{0}+\mathbf{1 8 5 . 8 4}=185.84 \\
\text { Cost }_{2} & =\min \left\{\text { Cost }_{0}+E_{02}^{0}, \text { Cost }_{1}+E_{12}^{0}\right\} \\
& =\min \{\mathbf{0}+\mathbf{3 5 8 . 3 1}, 185.84+230.87\}=358.31 \\
\text { Cost }_{3} & =\min \left\{\text { Cost }_{0}+E_{03}^{0}, \text { Cost }_{1}+E_{13}^{0}, \text { Cost }_{2}+E_{23}^{0}\right\} \\
& =\min \{0+550.41, \mathbf{1 8 5 . 8 4}+\mathbf{3 6 1 . 9 9}, 358.31+207.92\}=547.83 \\
\text { Cost }_{4} & =\min \left\{\text { Cost }_{0}+E_{04}^{0}, \text { Cost }_{1}+E_{14}^{0}, \text { Cost }_{2}+E_{24}^{0}, \text { Cost }_{3}+E_{34}^{0}\right\} \\
& =\min \{0+995.64,185.84+730.86,358.31+473.31, \mathbf{5 4 7 . 8 3}+\mathbf{2 8 1 . 5 9}\}=829.42(5.42)
\end{aligned}
$$

To summarize, the optimal value of this problem is 829.42. The corresponding optimal plans of the two levels are also obtained as shown in Table 5.9. To remain that the fixed revenue of the manufacturer is not considered in the optimization.

\subsection{Numerical tests for the $2 U L S_{P(W C R)}$ model}

In this section, we show differences between results (optimal production plans and different costs) obtained by classic $M L L P$ model, $2 U L S_{P(W C R)}$ model with sequential approach and with centralized 
Table 5.9 - Optimal production plans of the illustrative example

\begin{tabular}{lllll}
\hline Period & 1 & 2 & 3 & 4 \\
\hline Demand & 10 & 20 & 15 & 30 \\
\hline Supplier & 10 & 35 & 0 & 30 \\
Manufacturer & 10 & 35 & 0 & 30 \\
\hline
\end{tabular}

approach. These differences illustrate the influence of considering the financing cost of OWCR on an optimal production program at both levels. In following tests, the optimal program using the traditional $M L L P$ model for two level problem is denoted as $P_{o p t}^{i M}$ ( $i=0$ for the supplier's plan and $i=1$ for the manufacturer's plan). Moreover, $P_{o p t}^{i S}$ and $P_{o p t}^{i C}$ respectively represents the optimal programs of the supplier (with $i=0$ ) and of the manufacturer (with $i=1$ ) calculated by $2 U L S_{P(W C R)}$ model with the sequential and the centralized approaches. The tests are organized as follows:

- Firstly, a comparison between $P_{o p t}^{i M}, P_{o p t}^{i S}$ and $P_{o p t}^{i C}$ with a same set of parameter values is provided to show the differences in the production programs;

- Secondly, a comparison of the logistic cost, financial cost, total cost of both levels and the revenue of the supplier between the results obtained by the sequential and the centralized approaches;

- Thirdly, the evolution of a production program following the variation of discount and interest rates respectively with the centralized approach;

- Lastly, the influence of the variation of the selling price of the supplier on the production plans with the centralized approach.

For the following tests, we adopt the demand of one instance of Dellaert et al. (2000) (ph2in01st1de01, demand 1) over 24 periods. Values of other parameters are given in Table 5.10). These values will be used by default if without further specification. The main feature of these inputs are:

- since the purchasing and production cost are not considered in traditional MLLP problem, these unit costs are set to zero in order to compare the optimal programs in the same conditions in test 1 . Accordingly, the delays in payment involved only in purchasing are thus irrelevant in determining the production planning. Thus, $a=p=r_{0}=0$ in test 1 as well as the unit selling price;

- to remind that, some parameters is set with respect to the assumptions:

- the discount rate is greater at the supplier level than the one of the manufacturer level;

- the inventory holding unit cost is greater at the manufacturer level than the one at the supplier level;

- the payment delay from the manufacturer is shorter or equal to the one from the supplier.

- the unit selling price of the manufacturer is given in the following table. However, the manufacturer's revenue is constant with a fixed unit price and fixed external demand. Thus, this unit price do not impact the production program. However, the unit price of the supplier should be taken into account and be tested;

— the delivery delay is not considered in the following tests.

\subsubsection{Test 1: production program comparisons}

We first compare optimal programs separately calculated with the classic $M L L P$ model, $2 U L S_{P(W C R)}$ model with sequential approach and with centralized approach. Difference between $P_{o p t}^{i M}, P_{o p t}^{i S}$ and $P_{o p t}^{i C}($ with $i=0,1)$ are respectively illustrated in Figure 5.6 and 5.7 by level. As mentioned above, in order to compare with the same parameters considered in the classic MLLP model, we thus set $a_{i}=p_{i}=v_{i}=0$ with $i=0,1$ and $r_{0}=0$ to focus on the setup cost and inventory holding cost. Moreover, since the purchasing cost is not considered in test 1 , the payment delay for the raw material, $r_{0}$ is thus irrelevant in this test. We also compare the total number of products held in the 
Table 5.10 - Parameter values for the 24 periods instance

\begin{tabular}{lll}
\hline Parameter & Supplier & Manufacturer \\
\hline Unit sales price & 35 & 50 \\
Production unit cost, $p_{i}$ & 3 & 3 \\
Setup cost, $s_{i}$ & 800 & 600 \\
Purchasing unit cost, $a_{i}$ & 3 & 35 \\
Inventory holding unit cost per period, $h_{i}$ & 1 & 2 \\
Discount rate, $\alpha$ & 0.05 & 0.01 \\
Interest rate, $\beta$ & 0.03 & 0.03 \\
Delays in payment & $r_{2}=r_{1}=2, r_{0}=1$ \\
\hline
\end{tabular}

inventory of both level as well as the number of setups in Table 5.11. As a result, we observe that

- through the plans obtained by MLLP model, we prefer to hold the finished products in the manufacturer than holding the semi-finished products in the supplier. This is because that the setup cost of the supplier is more expensive than the one of the manufacturer and the difference of unit holding cost between the two levels is relatively small. A quasi synchronized plannings of two level shown in Table 5.12 allows to reduce the inventory level at the supplier.

- comparing the plans without and with the financial aspects we consider in $2 U L S_{P(W C R)}$ problem, the number of setups is generally increased (from 5 to 6 at the supplier level and from 6 to 10 or 8 at the manufacturer level). This confirms the observation we found in the tests for single-level problem that we have more interest to produce with a smaller lot size in order to suffer less from the financial consequences;

- on the one hand, using the sequential approach will significantly reduce the manufacturer's inventory level for its prioritized optimization objective. On the other hand, the centralized approach balances the inventory levels between the two levels for a global optimization objective.

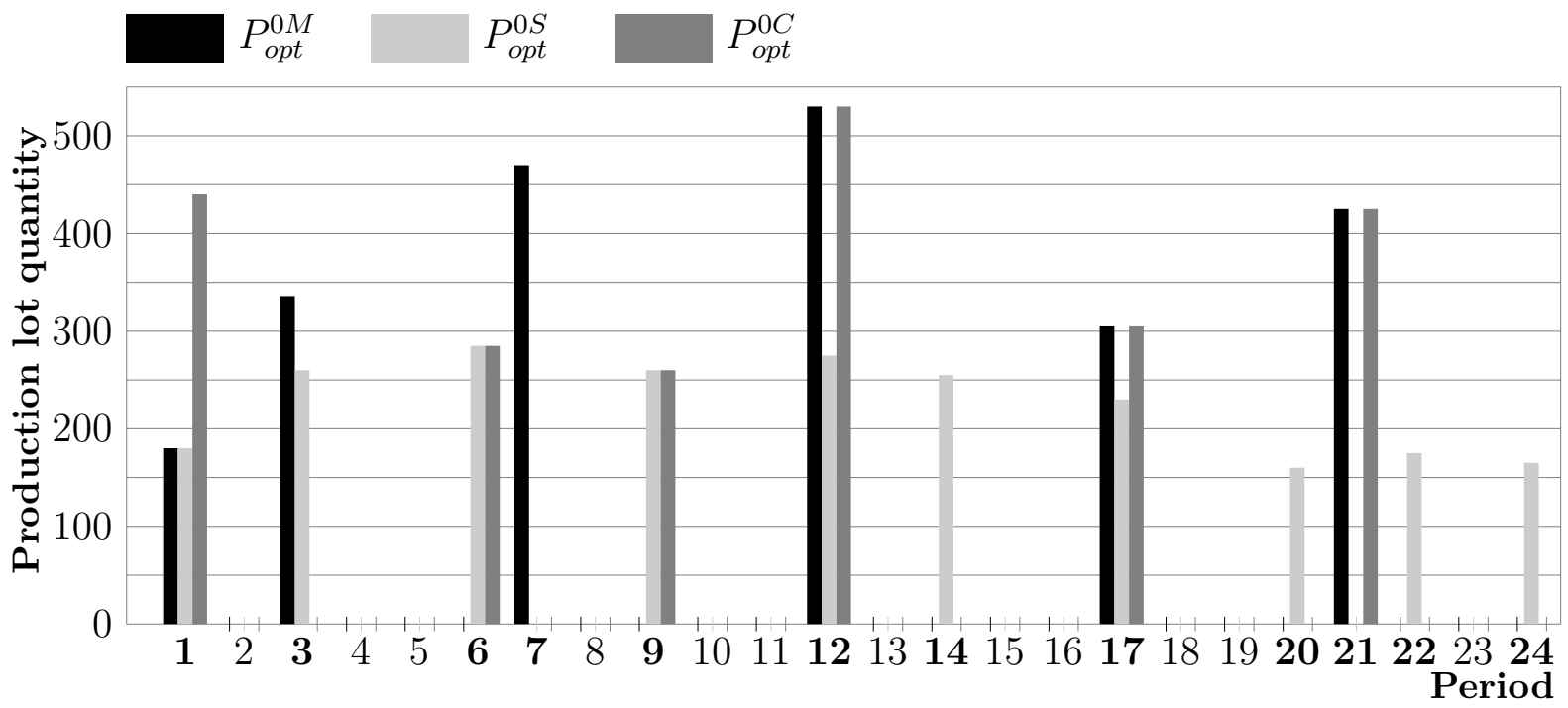

Figure 5.6 - Comparison of optimal production programs of the manufacturer with different approaches 


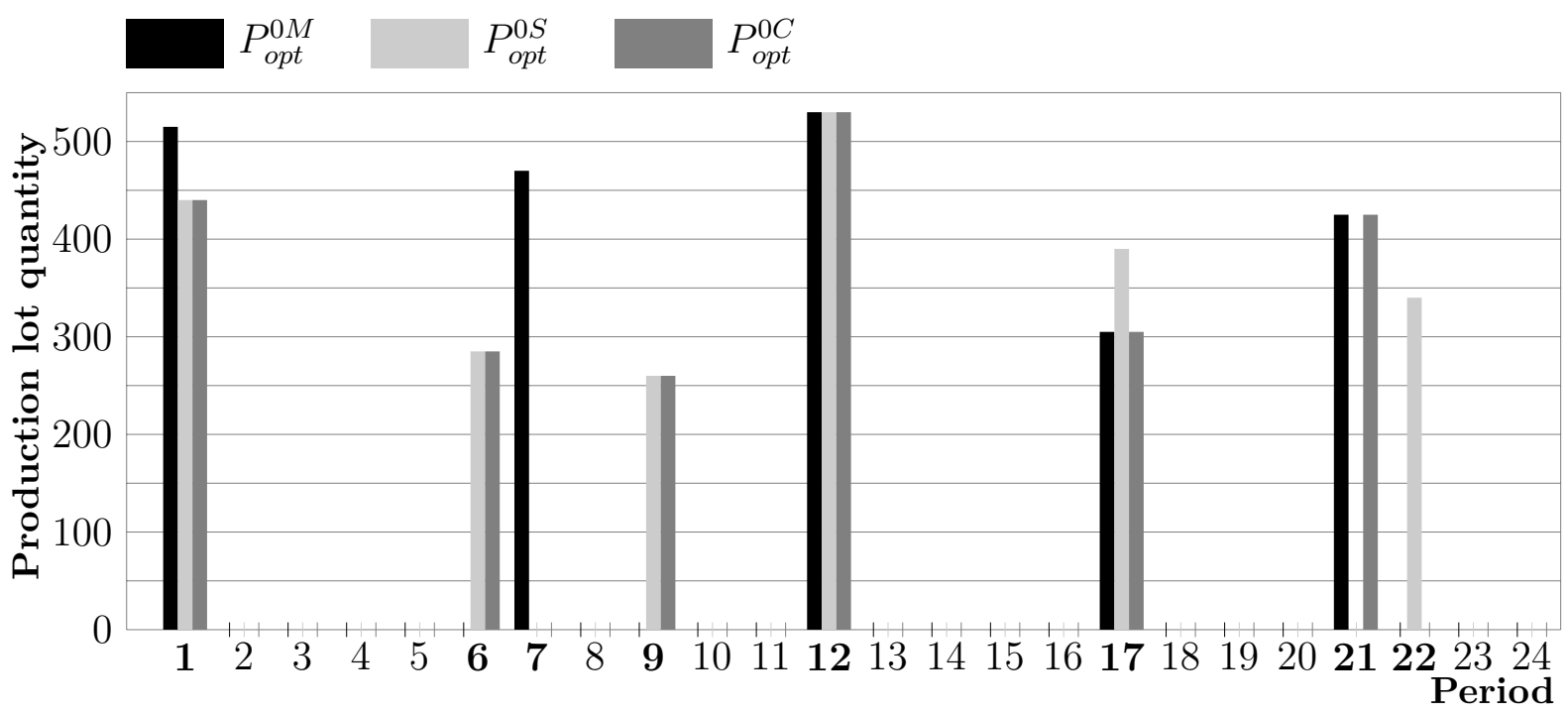

Figure 5.7 - Comparison of optimal production programs of the supplier with different approaches

Table 5.11 - Comparison of total products held in inventory over time and of the number of setups

\begin{tabular}{lcc}
\hline Approaches & Supplier & Manufacturer \\
\hline & Total products held (in unit/period) \\
\hline MLLP & 670 & 3085 \\
Sequential & 1840 & 1230 \\
Centralized & 1005 & 2065 \\
\hline \multicolumn{3}{c}{ Number of setups } \\
\hline Sequential & 6 & 6 \\
Centralized & 6 & 10 \\
\hline
\end{tabular}

Table 5.12 - Setup timings comparison between optimal programs

\begin{tabular}{lllllllllllllllllllllllll}
\hline Period & 1 & 2 & 3 & 4 & 5 & 6 & 7 & 8 & 9 & 10 & 11 & 12 & 13 & 14 & 15 & 16 & 17 & 18 & 19 & 20 & 21 & 22 & 23 & 24 \\
\hline$P_{\text {opt }}^{1 M}$ & 1 & & 1 & & & & 1 & & & & & 1 & & & & & 1 & & & & 1 & & & \\
$P_{\text {opt }}^{0 M}$ & 1 & & & & & & 1 & & & & & 1 & & & & & 1 & & & & 1 & & & \\
\hline
\end{tabular}



1 , represents $Y_{i t}=1$ which means that a setup is occurred in the period $t$ at the level $i$

\subsubsection{Test2: production program evolution with different ratios between two-level discount rates}

In previous test for the single-level problem, we have tested the production program evolution respectively following the variations of the discount rate and the interest rate. Generally, comparing with the optimal program with the classic MLLP model, we would naturally obtain the optimal programs with equal or small lot size using either the sequential or the centralized approach. Therefore, in following test, we rather focus on the influence of the variation of the discount rate ratio between levels. Since we assume that $\alpha_{1}<\alpha_{0}$ in the chapter, we set the $\alpha_{1}$ at 0.01 and vary the $\alpha_{0}$ from 0.01 
to 0.09 in step of 0.02 . Values of other parameters are the same as in test 1 . We show the result of comparisons between the two approaches in Figure 5.8 and 5.9. Firstly, we compare the number of setups of the optimal program of both levels respectively with the two approaches. Observations can be summarized as follows:

- With the sequential approach, the manufacturer's plan remains the same since $\alpha_{1}$ is fixed. Then, the setups are more frequent when $\alpha_{1}$ is sufficiently greater. This complies with the observation we made in the test of the single-level problem.

- Moreover, using the centralized approach, the plans of the two levels synchronize when $\alpha_{0}=$ $\alpha_{1}=0.01$. However, when $\alpha_{0}$ increases, the change of plan occurs not necessarily at the supplier's level as shown in Figure 5.8. In this test, the lot size of the manufacturer decreases when the discount rate of the supplier increases for a centralized objective.

- Nevertheless, when $\alpha_{0}$ reaches 0.09 , its influence is expanded to both levels that we generally produce with smaller lots at both levels.
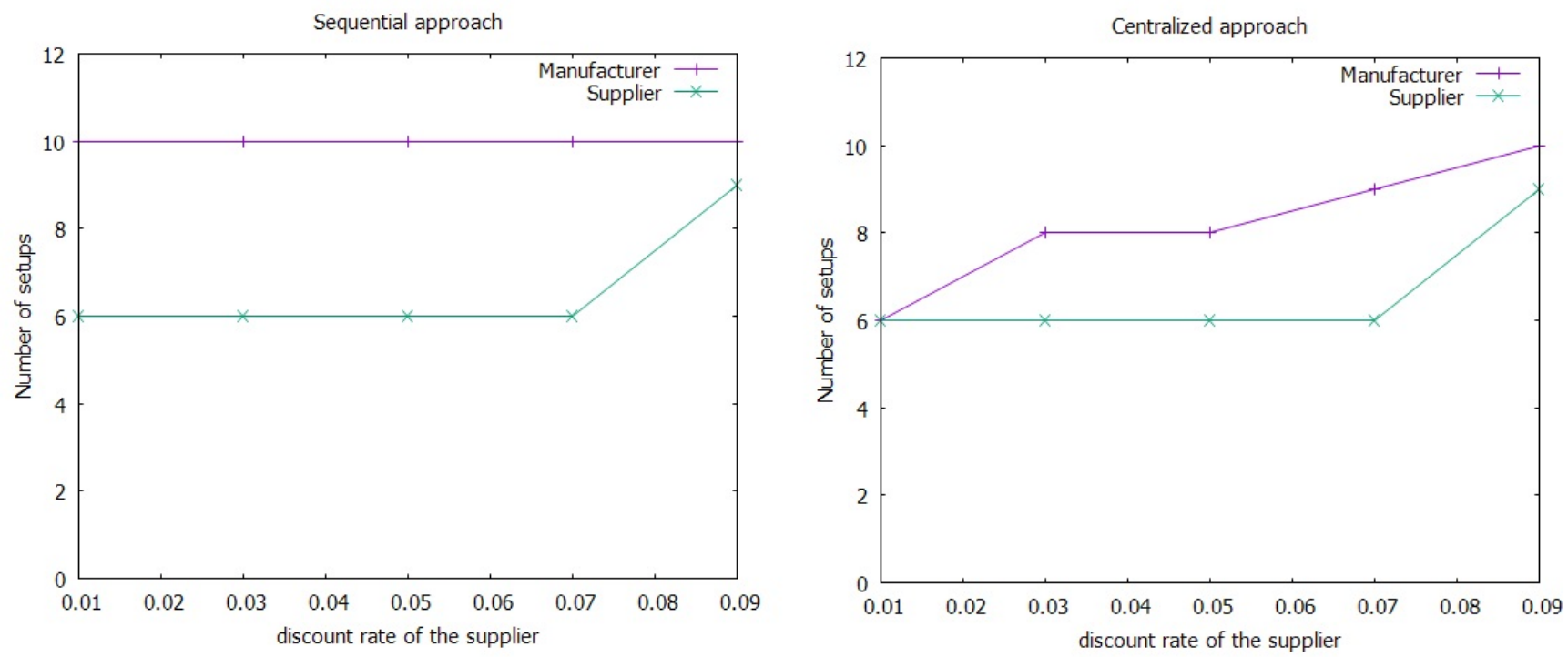

Figure 5.8 - Comparison of number of setups between optimal plans with different $\alpha_{0}$

Secondly, we focus on the difference of the cumulative inventory (denoted as $C I$ ) of both levels (i.e. total products held in inventory over the horizon, in "item/period") following the increase of $\alpha_{0}$. We observe that:

- there is not too many to state with the sequential approach. Only decrease of the CI level of the supplier is caused by the increase of the number of the setup following the increase of the $\alpha_{0}$.

- in the result with the centralized approach shown in 5.9, we find that, when $\alpha_{0}=0.01$, the CI level benefits from the synchronized plans and remains at zero over the planning horizon. With an increasing $\alpha_{0}$, the manufacture no longer advances too much its productions and let the semi-finished items stay longer in the supplier's inventory because the NPV of all costs of the supplier is smaller with an increased $\alpha_{0}$. Therefore, the supplier is interested to share the inventory stress from the manufacturer for a centralized objective. This trend continues until the $\alpha_{0}$ is greater enough $(\alpha=0.09)$, then the production of both levels are completely reorganized.

\subsubsection{Test3: program comparisons with different payment delays}

In following test, we focus on the influence of all payment delays on the two level plans with the two approaches: 

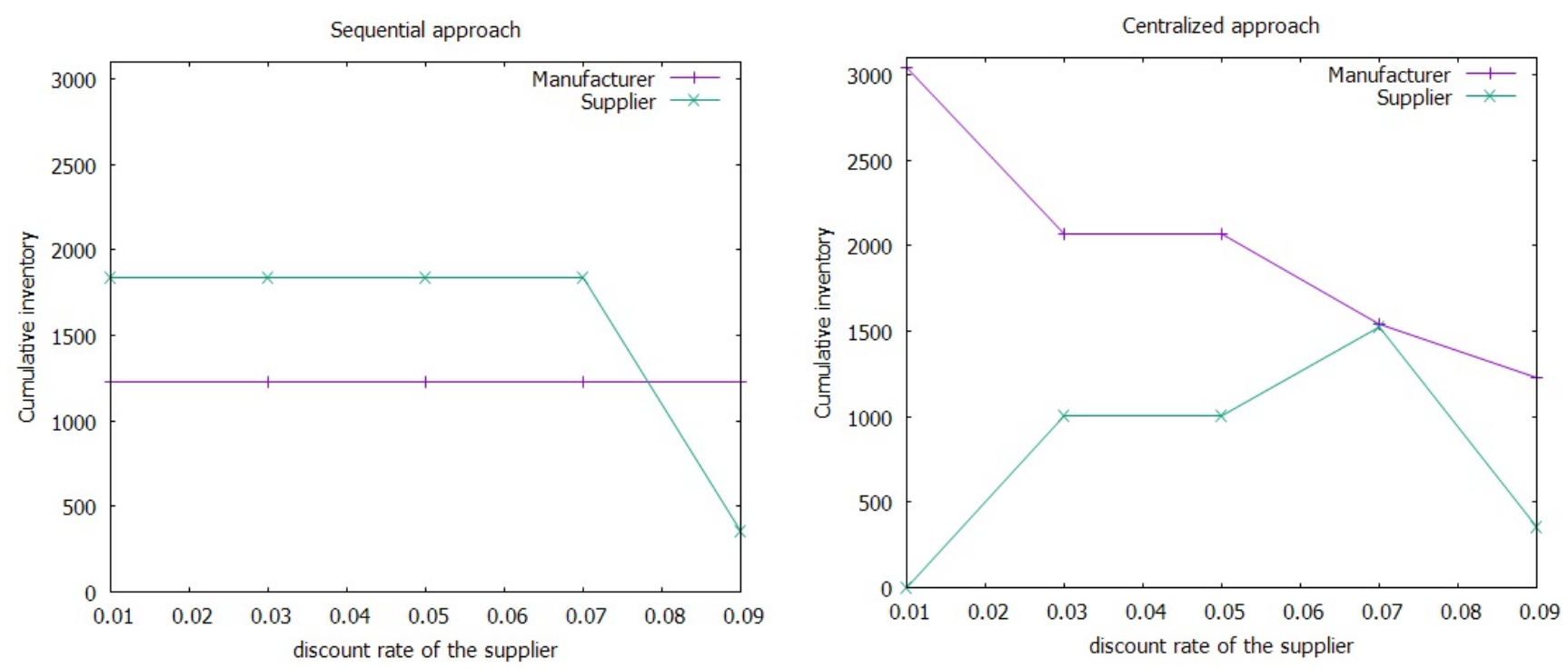

Figure 5.9 - Comparison of cumulative inventory between optimal plans with different $\alpha_{0}$

- with the sequential approach, we only investigate the variation of $r_{1}$ and $r_{2}$ because the variation of $r_{0}$ only change the WCR financial cost of the supplier. It is equal to the singlelevel problem that we have already tested in the previous chapter.

— with the centralize approach, we test the variation of all payment delays, i.e. $r_{0}, r_{1}$ and $r_{2}$.

Parameter values are adopted as in Table 5.10. We change $\beta_{0}$ and $\beta_{1}$ to 0.05 . In each series of test, the considered delay, $r_{i}$ with $i \in\{0,1,2\}$, varies from 2 to 18 in step of 8 .

firstly, we test with the varying $r_{2}$ using the two approaches. Since we assume that $r_{1} \geq r_{2}, r_{1}$ is thus set to 18 in this part of test and $r_{2}$ vary from 2 to 18 in step of 8 .

- with the sequential approach, we confirm the finding in Section 4.6.6 that the increase of the payment delay from the client decreases the number of setups of the optimal program through the result presented in left side of the Figure 5.10. Moreover, as shown in Table 5.13 , this decrease of number of setups at the manufacturer level may lead to a decrease of the NPV of supplier's total costs. The reason of this decrease is that the productions of the external demand in manufacturer's site are generally advanced caused by the decrease of the number of setups. Therefore, the supplier will receive some payments from the manufacturer in an early period. Its WCR will thus be reduced as well as the associated WCR costs. In the meantime, the revenue of the supplier increases for the same reason. Consequently, the profit of the supplier increases. Same results are also found in other tests, e.g., with $h_{0}=3$. To sum up, the increase of the $R_{2}$ consequently decreases the lot size of the manufacturer's production, then potentially influences the profit of the supplier in a positive way. Besides, the negative profit only results from the choice of the selling price of the supplier and $r_{1}=18$ that significantly discounts the revenue.

- with the centralized approach, we shown the number of setups in Figure 5.10 following the variation of $r_{2}$. Same increase of the supplier's profit is observed in this centralized case. In addition, we confirm that the centralized approach favors the supplier's profit through a smaller total cost and a higher revenue compared with the result in Table 5.13. However, it may cause a higher total cost of the manufacturer for the centralized objective.

Secondly, we test with the varying $r_{1}$ using both approaches with $r_{2}=2$ and $r_{0}=1$.

- with the sequential approach, the variation of $r_{1}$ decrease the number of setups of the supplier's optimal as shown in Figure 5.11. It confirms the observation in Section 4.6.6. However, the manufacturer's plans are still the same with the three $r_{1}$. The reason is explained in Test 4.6.4 that the downstream payment delay only involves in the purchasing cost and its associated 

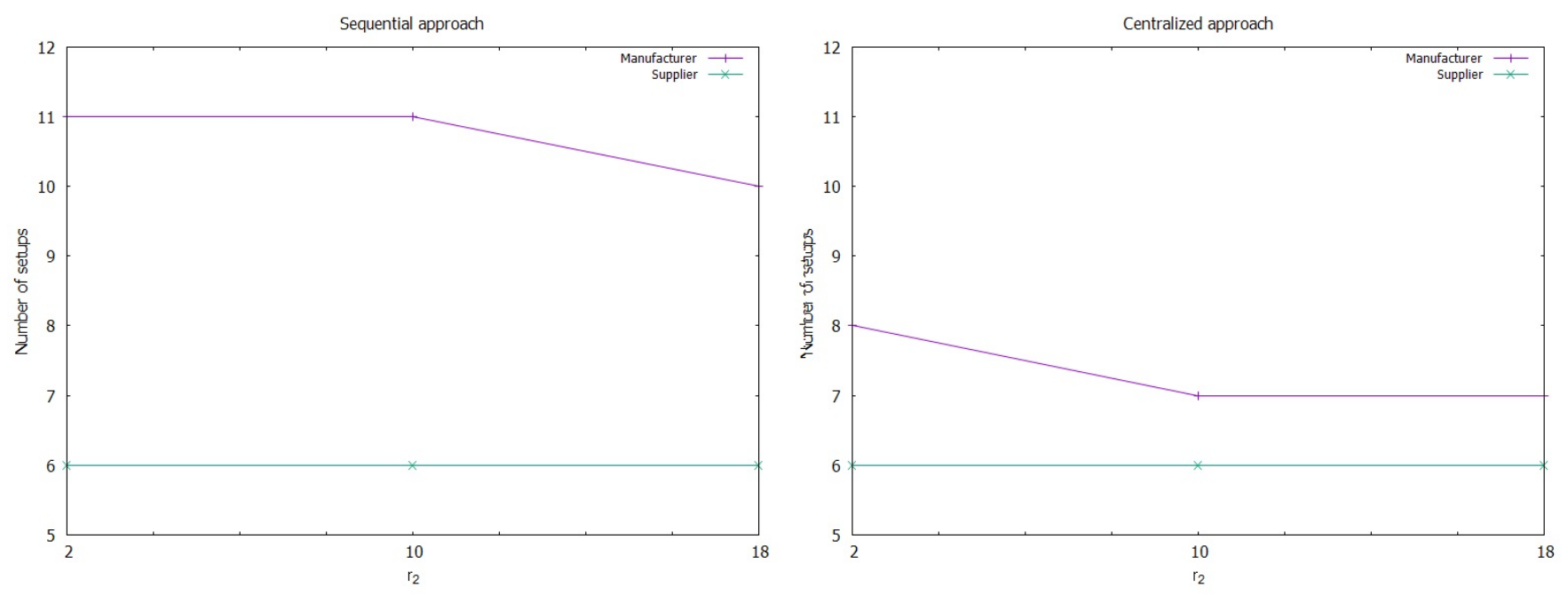

Figure 5.10 - Production plan comparison with varying $r_{2}$

Table 5.13 - Comparison of NPVs of financial terms with different $r_{2}$ using the sequential approach

\begin{tabular}{l|ccc|c}
\hline & \multicolumn{3}{|c|}{ Supplier } & Manufacturer \\
\hline$r_{2}$ & Total costs & Revenue & Profit & Total cost \\
\hline 2 & 21530.7 & 20156.5 & -1374.2 & 23465.4 \\
10 & 21530.7 & 20156.5 & -1374.2 & 54918.8 \\
18 & 21290.1 & 20307.4 & -982.7 & 83908 \\
\hline
\end{tabular}

Table 5.14 - Comparison of NPVs of financial terms with different $r_{2}$ using the centralized approach

\begin{tabular}{l|ccc|c}
\hline & \multicolumn{3}{|c|}{ Supplier } & Manufacturer \\
\hline$r_{2}$ & Total costs & Revenue & Profit & Total cost \\
\hline 2 & 19462.3 & 20749.4 & 1287.1 & 24943.8 \\
10 & 18904.5 & 20954.5 & 2050 & 56904.8 \\
18 & 18904.5 & 20954.5 & 2050 & 85686.5 \\
\hline
\end{tabular}

WCR financial cost that has only a small impact on the optimal plan.

- similar changes are found using the centralized approach. Moreover, a significant decrease of the supplier's profit are found with both approaches as presented in Table 5.15 and 5.16. It is mainly due to the enlarged payment delay from the manufacturer (i.e. $r_{1}$ ).

Table 5.15 - Comparison of NPVs of financial terms with different $r_{1}$ using the sequential approach

\begin{tabular}{l|ccc|c}
\hline & \multicolumn{3}{|c|}{ Supplier } & Manufacturer \\
\hline$r_{1}$ & Total costs & Revenue & Profit & Total cost \\
\hline 2 & 14691.5 & 43999 & 29307.5 & 85056.6 \\
10 & 18817.5 & 29780.3 & 10962.8 & 53035.9 \\
18 & 21530.7 & 20156.5 & -1374.2 & 23465.4 \\
\hline
\end{tabular}

Lastly, $r_{0}$ is varied from 2 to 18 and other parameters are adopted from the Table 5.10. As illustrated in Figure 5.12, the small impact of the downstream payment delay do not change the optimal plans in the three cases. However, it still decreases the total cost of the supplier because it shortens the financing duration for its logistic costs and decrease the WCR financial cost. Consequently, the 



Figure 5.11 - Production plan comparison with varying $r_{1}$

Table 5.16 - Comparison of NPVs of financial terms with different $r_{1}$ using the centralized approach

\begin{tabular}{l|ccc|c}
\hline & \multicolumn{3}{|c|}{ Supplier } & Manufacturer \\
\hline$r_{1}$ & Total costs & Revenue & Profit & Total cost \\
\hline 2 & 13136.9 & 45293.3 & 32156.4 & 86848.2 \\
10 & 16937.6 & 30656.3 & 13718.7 & 54664.7 \\
18 & 19462.3 & 20749.4 & 1287.1 & 24943.8 \\
\hline
\end{tabular}

supplier's profit increases.
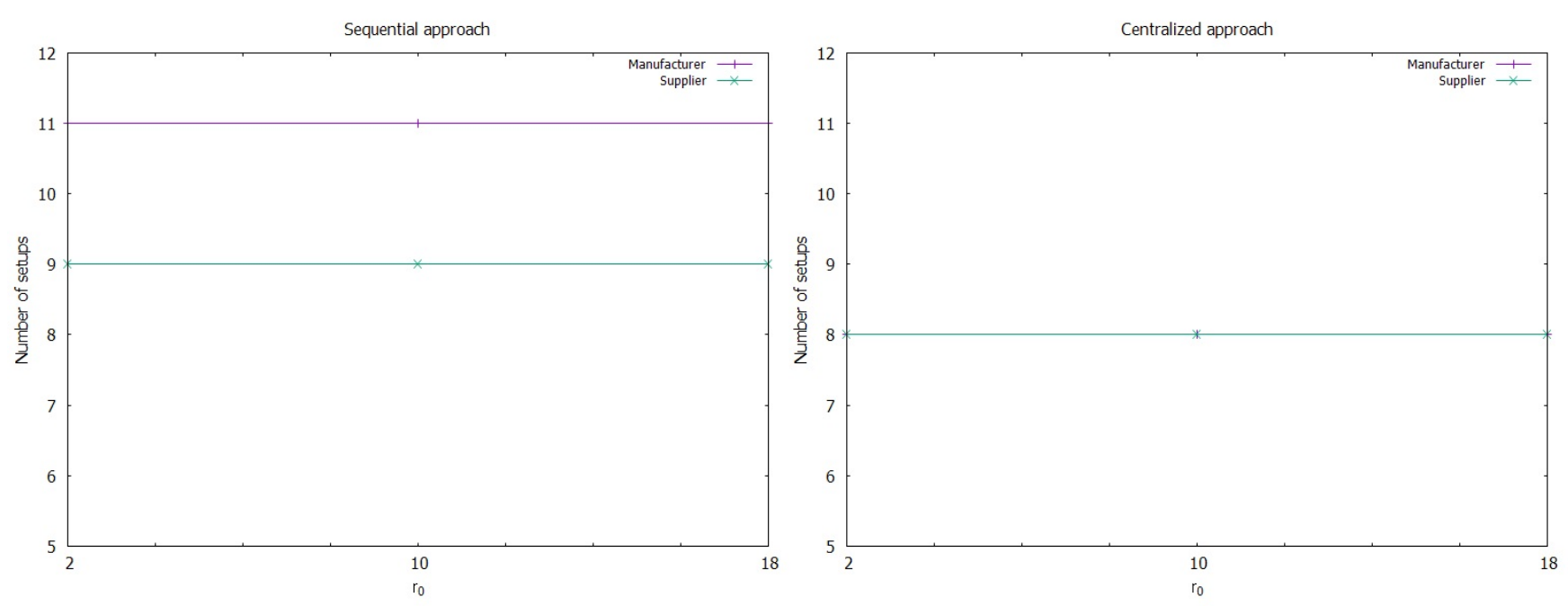

Figure 5.12 - Production plan comparison with varying $r_{0}$

Furthermore, focusing on the centralized approach, a comparison of the supplier's profit (Profit $\left.{ }_{0}\right)$, the manufacturer's total cost $\left(T C_{1}\right)$ and the objective value $(O V)$ changes between $r_{i}=2$ and $r_{i}=18$ $(i \in\{0,1,2\})$ is given in Table 5.19. For example, $\Delta$ Profit $_{0}=$ Profit $_{0}\left(r_{i}=18\right)-$ Profit $_{0}\left(r_{i}=2\right)$. To remind that $O V$ represent the sum of the logistic costs and the WCR financial costs of both levels minus the supplier's revenue. Since we attempt to minimize the $O V$, it is obvious that $r_{2}$ should be shorten as much as possible in order to reduce the unnecessary WCR financial cost of the manufacturer. Moreover, it is beneficial to lengthen $r_{1}$ and $r_{0}$ that may further decrease the OV. 
Table 5.17 - Comparison of NPVs of financial terms with different $r_{0}$ using the sequential approach

\begin{tabular}{l|ccc|c}
\hline & \multicolumn{3}{|c|}{ Supplier } & Manufacturer \\
\hline$r_{0}$ & Total costs & Revenue & Profit & Total cost \\
\hline 2 & 14300.1 & 43999 & 29698.9 & 85056.6 \\
10 & 11770.5 & 43999 & 32228.5 & 85056.6 \\
18 & 10058.3 & 43999 & 33940.7 & 85056.6 \\
\hline
\end{tabular}

Table 5.18 - Comparison of NPVs of financial terms with different $r_{0}$ using the centralized approach

\begin{tabular}{l|ccc|c}
\hline & \multicolumn{3}{|c|}{ Supplier } & Manufacturer \\
\hline$r_{0}$ & Total costs & Revenue & Profit & Total cost \\
\hline 2 & 12738.9 & 45293.3 & 32554.4 & 86848.2 \\
10 & 10167 & 45293.3 & 35126.3 & 86848.2 \\
18 & 8426.2 & 45293.3 & 36867.1 & 86848.2 \\
\hline
\end{tabular}

Table 5.19 - Comparison of changes with the variations of payment delays using the centralized approach

\begin{tabular}{c|ccc}
\hline Varying parameter & $\Delta$ Profit $_{0}$ & $\Delta$ TC $_{1}$ & $\Delta O V$ \\
\hline$r_{2}$ & 762.9 & 60742.7 & 59979.8 \\
$r_{1}$ & -30869.3 & -61904.4 & -31035.1 \\
$r_{0}$ & 4321.69 & 0 & -4312.7 \\
\hline
\end{tabular}

With the increasing the $r_{1}$, the decrease of the OV mainly results from the decreased total cost of the manufacturer and the decrease of the supplier's profit. However, the increasing $r_{0}$ only influences the supplier's profit in a positive way that decreases the OV. According to the amplitude of these changes, from the management point of view of each site, it should preferentially ensure the upstream payment that more significantly impact its profit and the global profit than the changes brought by the downstream payment. It is reinforced by the fact that the WCR financial cost of all operation costs depends on the upstream payment delay that should be managed as a higher-priority task. In addition, from the global point of view, since the payment delay between sites conversely impacts the two site, it should be carefully managed and certain negotiation mechanisms may be involved in order to satisfy both sites.

\subsubsection{Production program evolution with different unit selling prices of the supplier}

In single-level problem, the revenue does not change with a deterministic and fixed the external demand. Therefore, the unit selling price gives no influence in determining the optimal production plan. However, it is no longer the case in this two-level problem because the demand of the supplier is the production plan of the manufacturer and the unit selling price of the supplier is the unit purchasing cost of the manufacturer. Consequently, the supplier's revenue depends on the manufacturer's plan considering the discount effect. In following tests, the $v_{0}\left(=a_{1}\right)$ is varied from 35 to 65 in step of 15. Other parameter values are the same as in Table 5.10 .

- with the sequential approach, the increase of $v_{0}$ (i.e. $a_{1}$ ) will decrease the production lot sizes in the optimal plan of the manufacturer and the optimal plans of the supplier remain the same in the three cases. This observation corresponds to the result presented in Section 4.6.4. As a result of the increase of setups at the manufacturer level, the payments from the manufacturer 
to the supplier are postponed. This delay leads to a increase of the WCR financial cost of the supplier.

- subsequently, the influence of the increase of $v_{0}$ on the optimal plans within the centralized approach is tested. The optimal plans of both levels are presented in Figure 5.13 and 5.14 . Generally, an increase of the number of setups is found on both levels. The increase of setups of the manufacturer's level confirms the finding in Section 4.6.4. As a consequence of the centralized objective, the increase of the selling price will thus decrease the production quantities of the supplier. As shown in Table 5.20, the increase of $v_{0}$ will obviously increase the supplier's profit but more significantly increase the total cost of the manufacturer that globally increase the objective value. Therefore, pricing problem for the supplier should also consider the consequences at the manufacturer level with the centralized objective.

Table 5.20 - Comparison of the profit of the supplier, the total cost of the manufacturer and the objective value with different selling prices using the centralized approach

\begin{tabular}{c|ccc}
\hline Varying parameter & Profit $_{0}$ & $T_{1}$ & $O V$ \\
\hline$v_{0}=35$ & 32156.4 & 86848.2 & 54691.8 \\
$v_{0}=50$ & 51065.9 & 117431 & 66364.6 \\
$v_{0}=65$ & 68431.2 & 146412 & 77981.1 \\
\hline
\end{tabular}

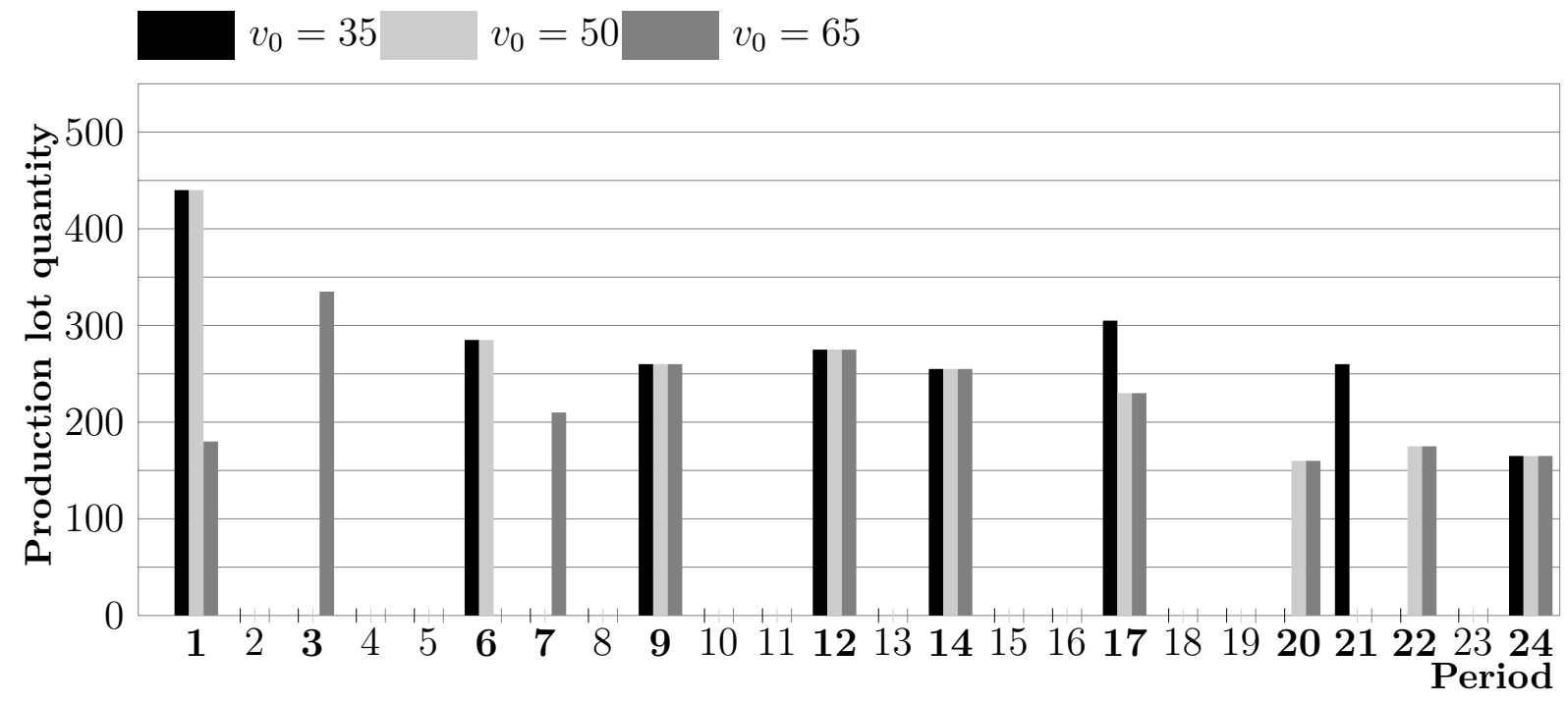

Figure 5.13 - Comparison of optimal production programs of the manufacturer with different selling prices of the supplier

\subsection{Generalization to multi-level problem}

In this section, the two-level problem is generalized to multi-level, namely $M L L P_{P(W C R)}$ problem. Since the sequential approach can be naturally extended to multi-level, we here focus on the centralized approach. To do so, first, the WCR model in multi-level case will be firstly given. Secondly, the ZIO property will be further examined for multi-level case. In the end of this section, the algorithm for $2 U L S_{P(W C R)}$ will be extended to the multi-level case. 


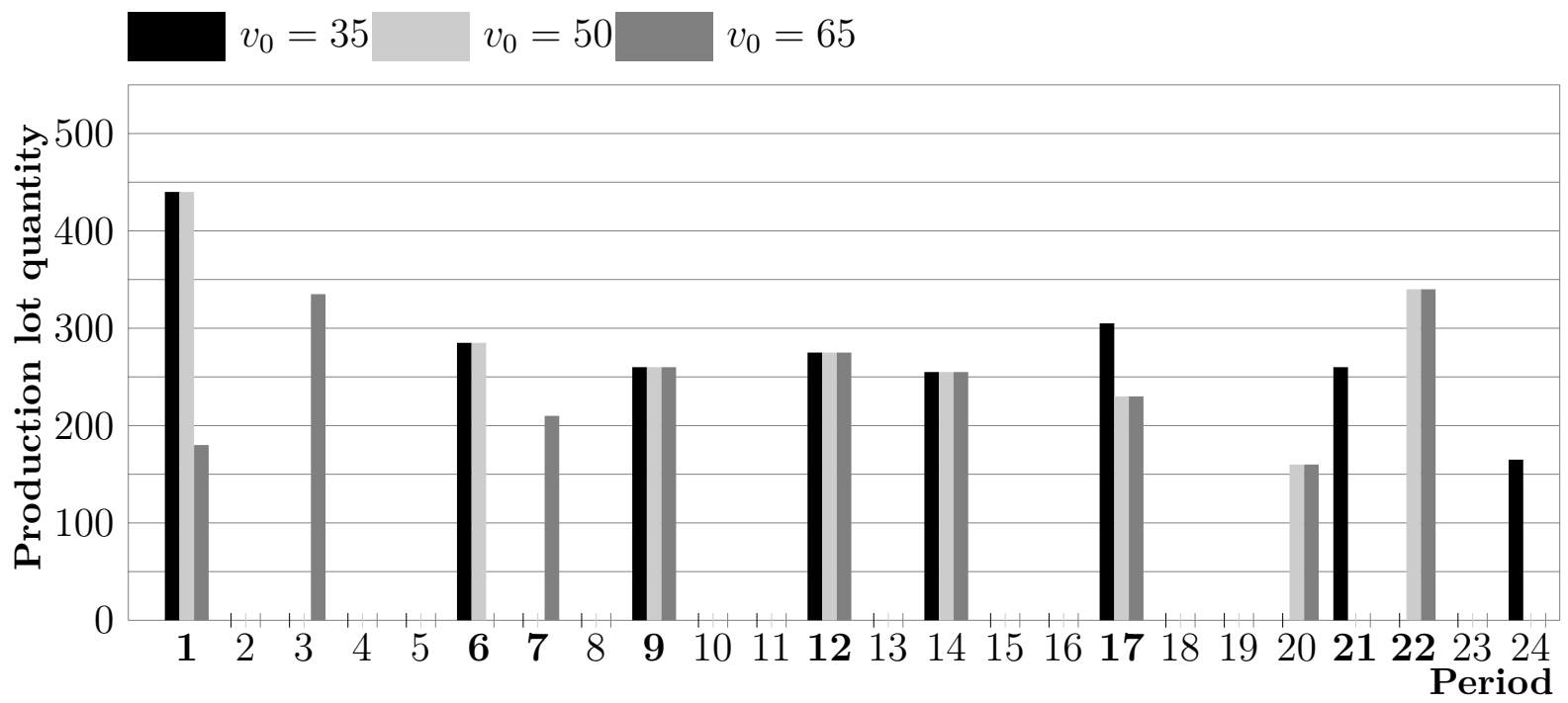

Figure 5.14 - Comparison of optimal production programs of the supplier with different selling prices of the supplier

\subsection{1 $M L L P_{P W C R}$ model and centralized approach}

In multi-level case, we tackle the system with a serial structure which is the same case presented in Zangwill (1966). Therefore, we here consider a $N$ level supply chain and in order to provide the final product, several processes are required and respectively executed by a site at different level. We additionally assume that only one process takes place in one facility. For example, the site 0 processes the raw material and delivers the output to the second site for further manufacturing until the final product is completed in the site $N-1$ for satisfying the external demand. Following the fixed sequence with which the physical flow passes through the chain, we thus assume that the site $i$ always provides the input of the site $i+1$. Only the site $N-1$ directly responds to the final customer. The same assumption is made as for the two-level problem in section 5.3.1. As stated in Zangwill (1966), the site $i$ holds its output in its own inventory and delivers it only when it is needed as input to the site $i+1$. In the next paragraph, the WCR model for this case is provided with the same assumptions as for two-level case.

\subsubsection{WCR model in multi-level}

In multi-level case, the notation of the parameters and the decision variables are identical to the same for the two-level case, see in Table 5.3 and 5.4. We add $N$ for the number of levels and index $i \in 0, \ldots, N-1$. Furthermore, the financial need of the site $i$ to cover its logistic cost is only caused by the payment its supplier at level $i-1$ before the collection of payments from its customer at level $i+1$. Following the concept of the WCR model in two-level case, the general WCR model can be formulated as follows. The cumulation of the discount effect caused by the delivery delay is represented by the first term in the following formulation, $\prod_{l=0}^{i} \frac{1}{\left(1+\alpha_{i}\right)^{L_{l}}}$.

$$
\begin{gathered}
W C R_{i t k}^{\text {purch }}=\prod_{l=0}^{i} \frac{1}{\left(1+\alpha_{i}\right)^{L_{l}}} \cdot a_{i} \cdot X_{i t k} \cdot\left(\sum_{j=t+r_{i}}^{T} \frac{1}{\left(1+\alpha_{i}\right)^{j}}-\sum_{\substack{j=k+L_{i+1} \\
+r_{i+1}}}^{T} \frac{1}{\left(1+\alpha_{i}\right)^{j}}\right) \\
W C R_{i t k}^{\text {prod }}=\prod_{l=0}^{i} \frac{1}{\left(1+\alpha_{i}\right)^{L_{l}}} \cdot p_{i} \cdot X_{i t k} \cdot \sum_{j=t}^{\substack{k+L_{i+1} \\
+r_{i+1}-1}} \frac{1}{\left(1+\alpha_{i}\right)^{j}}
\end{gathered}
$$




$$
\begin{gathered}
W C R_{i t k}^{\text {setup }}=\prod_{l=0}^{i} \frac{1}{\left(1+\alpha_{i}\right)^{L_{l}}} \cdot \frac{s_{i} \cdot Y_{i t}}{Q_{i t}+1-Y_{i t}} \cdot X_{i t k} \cdot \sum_{j=t}^{\substack{k+L_{i+1} \\
+r_{i+1}-1}} \frac{1}{\left(1+\alpha_{i}\right)^{j}} \\
W C R_{i t k}^{i n v}=\prod_{l=0}^{i} \frac{1}{\left(1+\alpha_{i}\right)^{L_{l}}} \cdot h_{i} \cdot X_{i t k} \cdot \sum_{w=t}^{k-1} \sum_{j=w}^{k+r_{i+1}-1} \frac{1}{\left(1+\alpha_{i}\right)^{j}}
\end{gathered}
$$

The total WCR for a site $i \in\{0,1\}$ is in the same equation as the equation (5.9)

$$
W C R_{i}=\sum_{t=1}^{T} \sum_{k=t}^{T}\left(W C R_{i t k}^{\text {purch }}+W C R_{i t k}^{\text {prod }}+W C R_{i t k}^{\text {setup }}+W C R_{i t k}^{i n v}\right)
$$

\section{Mathematical formulation of $M L L P_{P W C R}$ model}

In addition to the up-mentioned WCR model, the logistic cost is also composed of the following terms:

$$
\begin{gathered}
L C_{i}^{\text {purch }}=\prod_{l=0}^{i} \frac{1}{\left(1+\alpha_{i}\right)^{L_{l}}} \cdot a_{i} \sum_{t=1}^{T} \frac{Q_{i t}}{\left(1+\alpha_{i}\right)^{t+r_{i}}} \\
L C_{i}^{\text {prod }}=\prod_{l=0}^{i} \frac{1}{\left(1+\alpha_{i}\right)^{L_{l}}} \cdot p_{i} \cdot \sum_{t=1}^{T} \frac{Q_{i t}}{\left(1+\alpha_{i}\right)^{t}} \\
L C_{i}^{\text {setup }}=\prod_{l=0}^{i} \frac{1}{\left(1+\alpha_{i}\right)^{L_{l}}} \cdot s_{i} \cdot \sum_{t=1}^{T} \frac{Y_{i t}}{\left(1+\alpha_{i}\right)^{t}} \\
L C_{i}^{\text {inv }}=\prod_{l=0}^{i} \frac{1}{\left(1+\alpha_{i}\right)^{L_{l}}} \cdot h_{i} \cdot \sum_{t=1}^{T} \sum_{k=t}^{T} \sum_{m=t}^{k-1} \frac{X_{i t k}}{\left(1+\alpha_{i}\right)^{m}}
\end{gathered}
$$

The general mathematical formulation of the centralized approach for multi-level problem can thus be written as:

$$
\begin{array}{ccl}
\text { Max } & \sum_{i=0}^{N-1}\left[R_{i}-\left(L C_{i}+\beta_{i} W C R_{i}\right)\right] \\
\text { s.t. } & & \\
& I_{i, t+L_{i}-1}+Q_{i t}-I_{i, t+L_{i}} & \\
& & \forall i \in[0, N-1] \\
M Y_{i t}-Q_{i t} \geq 0 & \forall t \in\left[T_{i}+1, T_{i}+T\right] \\
Q_{i t}, I_{i, t+L_{i}} \geq 0 & \forall(i, t) \in[0, N-1] \times[1, T] \\
& \forall i \in[0, N-1] \\
Y_{t}=0 \text { or } 1 & \forall t \in\left[T_{i}+1, T_{i}+T\right] \\
& \forall(i, t) \in[0, N-1] \times[1, T]
\end{array}
$$

\section{ZIO property in $M L L P_{P(W C R)}$}

In this multi-site context, the payments of one site $i$ only involve its two adjacent levels, $i-1$ and $i+1$, in the supply chain. Therefore, we adopt similar concepts of proof as in previous proof for $2 U L S_{P(W C R)}$. The transformation into ZIO-structure plan can generally occurs either in one of the two extreme levels (i.e. 0 or $N-1$ ) or one of the other intermediate levels, $i$ with $i \in[1, N-2]$.

- The former cases are exactly the same cases that we investigate in the proof for $2 U L S_{P(W C R)}$;

- Thus, only the latter case needs to be studied. In this case, a transformation is made at level $i$ in order to change the plan into ZIO structure at one period. However, this transformation does not affect the revenue and all costs of its "client" at level $i+1$. This case becomes the second case we examine in the previous proof. 
To conclude, the ZIO property remains valid in this multi-level problem with the same assumptions made for two-level problem. Overall, we also favor the ZIO type plans in this multi-level problem.

\section{Generalization of two-level algorithm for $M L L P_{P(W C R)}$}

The shortest path type algorithm for two level problem can naturally be extended to multi-level. However, the difference in the concept of the algorithm is found in the formulation of arc value. In multi-level case, the interdependency between levels should be carefully considered. Therefore, there are three different types of formulation that need to be distinguished in the extension of the Figure 5.5 one for the site only has a predecessor and no successor (the site $N-1$ ), one site only has a successor and no predecessor (the site 0) and the other intermediate sites have both.

- For the site 0, we still consider it as a master problem and the formulation of the arc value remains the same as in two-level problem as well as the recursion:

- Arc value: $E_{t k}^{0}=\left(a_{0, t+r_{f}}^{\prime}+p_{0 t}^{\prime}\right) \sum_{l=t}^{k-1} d_{l}+s_{0 t}^{\prime}+O p t_{t k k}^{1}$

- Recursion: $O p t_{t}^{0}=\min _{j \in[0, t-1]}\left\{O p t_{j}^{0}+E_{j t}^{0}\right\}$

- For a intermediate site $i$ where $i=1, \ldots, N-2$, in order to take into account the interdependency between levels, we should add not only the holding cost and the financial cost of arc $t^{\prime}$ to $k^{\prime}$ at level $i-1$ (the first two terms in $E_{t t^{\prime} k^{\prime}}^{i}$ ) and the purchasing, setup and production cost of its own (the three following terms), but also the optimal value of the associated subproblem at level $i+1$ (the last one). Therefore, we still need a four index parameter that provides all necessary information for WCR calculations. Therefore, the arc value and the recursion of arcs at level $i$ are written as :

- Arc value: $E_{t k t^{\prime} k^{\prime}}^{i}=I n v_{t t^{\prime} k^{\prime}}^{i-1}+\beta_{i-1} W C R_{t k t^{\prime} k^{\prime}}^{i-1}-R_{t^{\prime} k^{\prime}}^{i-1}+\left(a_{i, t^{\prime}+r_{1}}^{i}+p_{i t^{\prime}}^{i}\right) \sum_{l=t^{\prime}}^{k^{\prime}-1} d_{l}+s_{i t^{\prime}}^{i}+O p t_{t^{\prime} k^{\prime} k^{\prime}}^{i+1}$

- Recursion: $O p t_{t k k^{\prime}}^{i}=\min _{t^{\prime} \in[t, t-1]}\left\{O p t_{t k t^{\prime}}^{i}+E_{t k t^{\prime} k^{\prime}}^{i}\right\}$

- For the site $N-1$, it only responds to the external demand. No change is necessary for the formulation of its arc value and of the recursion (where $L C_{t^{\prime} k^{\prime}}^{N-1}$ is in the same form of the equation (5.32)):

- Arc value: $E_{t k t^{\prime} k^{\prime}}^{N-1}=\operatorname{Inv} v_{t t^{\prime} k^{\prime}}^{N-2}+\beta_{N-2} W C R_{t k t^{\prime} k^{\prime}}^{N-2}-R_{t^{\prime} k^{\prime}}^{N-2}+L C_{t^{\prime} k^{\prime}}^{N-1}+\beta_{N-1} W C R_{t^{\prime} k^{\prime}}^{N-1}$

- Recursion: $O p t_{t k k^{\prime}}^{N-1}=\min _{t^{\prime} \in\left[t, k^{\prime}-1\right]}\left\{O p t_{t k t^{\prime}}^{N-1}+E_{t k t^{\prime} k^{\prime}}^{N-1}\right\}$

After formulating these arc values and the recursion for each level, a difficulty arises during the programming phase. It seems that the Russian doll type loop is complicate to be directly coded since the depth (levels) of the loop is only determined by the number of levels. Thus, the loop structure can not be built a priori in a general form without knowing the number of levels a prior. Moreover, all the optimal values and shortest paths at all levels should be dynamically kept for establishing the final optimal planning of all levels in processing this loop. For this reason, an algorithm reformulation is required in order to facilitate solving this problem. In the up-presented arc value formulation, we observe that, thanks to the ZIO property, all expressions of the three types of arc value are in function of the (entire) external demand of one or several periods. Therefore, it is possible to "peel off" the computation of all the shortest paths between each pair of nodes by level. For any level $i$ except for the level 0 , all paths as well as the optimal value are saved for further utilization as the result of the corresponding subproblem in calculation of the arc value at level $i-1$. For the level 0, we only need to keep one shortest path from the beginning of the planning horizon to the end. However, we must start the algorithm from the site $N-1$ at the bottom level who has only (one) 
predecessor.

The pseudo-code is presented as follows:

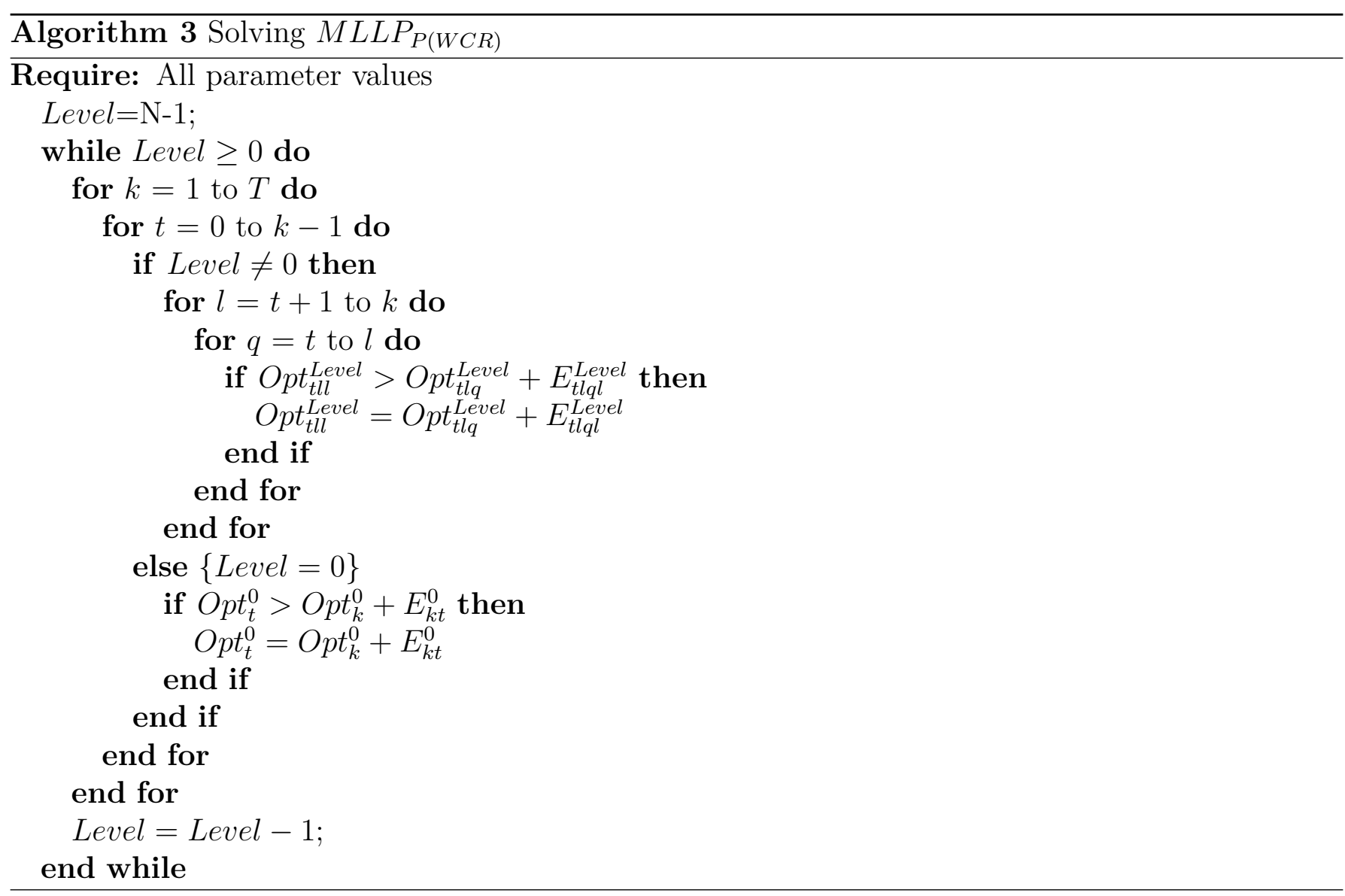

The loops $t$ and $k$ are for:

- generating all possible pairs of nodes for any level except for the level 0;

- the recursion of dynamic programming for the level 0 .

Then, the loops $l$ and $q$ are the recursion of the dynamic programming for other levels than level 0. In total, for each of these levels (other than 0 ), there are $O\left(T^{2}\right)$ pairs of nodes and each possible shortest path between one pair of nodes is determined in $O\left(T^{4}\right)$ because it is based on the single-level type algorithm. Besides, the resolution at level 0 takes $O\left(T^{2}\right)$ for one shortest path to solve. Globally, for this $N$ level problem, the overall complexity is $O\left(N T^{6}\right)$.

\subsection{Conclusions}

In this chapter, firstly, we extend the previous single-level problem to a supplier-manufacturer two-level problem with a serial chain structure. following similar steps in the previous chapter, after establishing the associated WCR model for this case, the corresponding mathematical model of this problem is established considering the WCR financial cost of the two levels. For the solution procedure, both the sequential approach, that prioritizes the maximization of manufacturer's profit than the supplier's, and the centralized approaches with a global profit maximization objective are proposed. For on thing, the sequential approach indeed consists in a direct application of the singlelevel algorithm at two levels. For another, it is proved that the ZIO property remains valid with certain assumptions for the centralized approach. This property allows us to develop a revised dynamic programming-based algorithm, in which the interdependency between levels is considered by the arc valuation. Through the numerical tests, the observations obtained in the tests of the single-level problem are confirmed. Moreover, other observations are related to the interdependency 
between levels. We found that the upstream payment delay and the selling price of the supplier should be preferentially managed.

Secondly, the generalization from the two-level problem to the multi-level problem is addressed. The mathematical model and the associated WCR model are further extended to the multi-level case as well as the two approaches and the proof of the ZIO property. In order to solve this multi-level problem with the centralized approach, the dynamic programming algorithm is modified to avoid the Russian doll type structure that is difficult to program. The new algorithm tackles the problem level by level and the final complexity of the algorithm is $O\left(N T^{6}\right)$ where $N$ represents the number of levels of the considered supply chain.

For future research, a generalization of the chain structure seems imperative. The first setup can focus on the assembly structure and then the general structure. Moreover, the development of heuristic or meta-heuristic algorithms helps us to solve problems with a more realistic size and with fewer assumptions. Furthermore, in addition to the two approaches proposed in this thesis, the decentralized approach has drawn increasing attention since the work of the Homberger (2010). The problem concerning the relation (domination) between supply chain members will thus be addressed that involves more negotiation mechanisms and game theory techniques as studied in work of Hovelaque et al. (2015). 



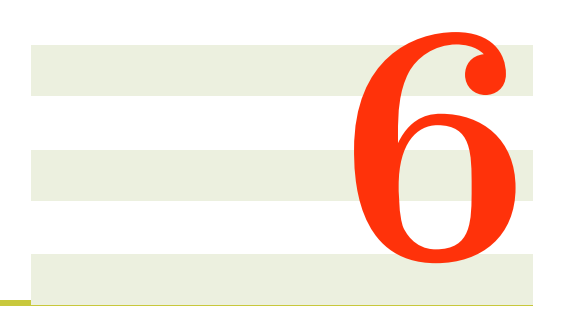

\section{Conclusion and future directions}

\section{Conclusion}

In this thesis, we introduce a first link between tactical planning models and the financial aspects of working capital requirements (WCR). Moreover, new generic WCR models are respectively proposed based on the EOQ, ULS and MLLP models. After integrating the WCR financial cost into these classic models, the financial consequences of the traditional logistic costs on the production planning are analyzed for each case.

In the first chapter, the research background and the motivation of our work are provided. We stress that the joint consideration of the physical and financial flows in the supply chain ensures a better alignment with the global objective of the company and improve the competitiveness in the increasing global market.

In this second chapter, to begin with, a new generic model of operations-related working capital requirements in the EOQ context is presented. Such a model allows us to measure the evolution of the WCR over the entire planning horizon. Furthermore, an EOQ-based profit maximization model is developed considering the financing cost of the WCR. The analytical formula of the optimal EOQ is derived and associated analysis are provided to highlight some managerial insights. It is shown that an increase in purchasing, production unit costs or the interest rate reduces the optimal production lot size in order to obtain the maximum profit. Moreover, it is demonstrated that the economic quantity proposed by our model is always smaller than the optimal quantity of the traditional EOQ model. Thus, it is desirable to launch more frequent production with smaller lots in this joint management case in order to reduce inventory levels and their associated holding costs. Additionally, a comparison with the approximation of Jesse et al. (1983) demonstrates that our model provides a more coherent and accurate measure of the optimal production quantity for a profit maximization objective when considering financial aspects. Finally, the numerical example illustrates the reduced sensitivity of our model to several parameters when compared with the classic EOQ model.

In the third chapter, firstly, the generic model of WCR is reformulated to adopt the discrete time horizon. Secondly, a ULS-based profit maximization model is developed considering both the financing cost of the WCR and the time value of money. This model is established for the single-site, single-level, single-product and infinite production capacity case. After proving the Zero-InventoryOrdering property for this problem, an exact method is developed based on dynamic programming. With this polynomial algorithm, we are able to not only reach the optimal plan which maximizes the net present value of profit, but also evaluate the profitability of satisfying a series of demands. 
Numerical tests are provided to show the interest of applying our WCR model through a comparison with the classical $U L S$ model. It is found that we have less interest in holding products in the inventory due to their dramatic amplification of financial costs.

In the fourth chapter, firstly, we extend the previous single-level problem to a supplier-manufacturer two-level problem with a serial chain structure. following similar steps in the previous chapter, after establishing the associated WCR model for this case, the corresponding mathematical model of this problem is established considering the WCR financial cost of the two levels. For the solution procedure, both the sequential approach, that prioritizes the maximization of manufacturer's profit than the supplier's, and the centralized approaches with a global profit maximization objective are proposed. For on thing, the sequential approach indeed consists in a direct application of the single-level algorithm at two levels. For another, it is proved that the ZIO property remains valid with certain assumptions for the centralized approach. This property allows us to develop a revised dynamic programming-based algorithm, in which the interdependency between levels is considered by the arc valuation. Through the numerical tests, the observations obtained in the tests of the single-level problem are confirmed. Moreover, other observations are related to the interdependency between levels. We found that the upstream payment delay and the selling price of the supplier should be preferentially managed.

Secondly, the generalization from the two-level problem to the multi-level problem is addressed. The mathematical model and the associated WCR model are further extended to the multi-level case as well as the two approaches and the proof of the ZIO property. In order to solve this multi-level problem with the centralized approach, the dynamic programming algorithm is modified to avoid the Russian doll type structure that is difficult to program. The new algorithm tackles the problem level by level and the final complexity of the algorithm is $O\left(N T^{6}\right)$ where $N$ represents the number of levels of the considered supply chain.

Besides, we are aware that this paper has some limitations as the first effort that theoretically considers the WCR cost in the classic dynamic lot-sizing problem. Immediate application for practical problem is still difficult. However, it will contribute to further studies in more complex and realistic cases as polynomial subproblems.

\section{Future directions}

In this thesis, an infinite production capacity and the constant value of parameters are assumed. Therefore, future works may naturally be extended to the case with varying parameter values and investigate whether the ZIO property is still valid for dynamic lot-sizing cases. Moreover, the consideration of the production capacity constraint is also an unavoidable extension of our work. A complexity analysis that reveals both its computational difficulty and some polynomial cases should be preformed. The classification proposed by Bitran \& Yanasse (1982) can be adopted to identify special cases. In addition, the financial capacity of the company is not considered in this thesis and a global financial resource is assumed to cover the WCR in the context of the project RSCM. Thus, the financial capacity can be taken into account as a model constraint. This financial capacity represents its cash flow reserve (free cash flow) for covering the production-related operation costs. Furthermore, this reserve can be provided by the debt holder (e.g., a bank) or by its own working capital (from its shareholders). Consequently, it raises a selection problem among all available financial resources as well as a problem of valuing their associated risks. Readers are referred to the work of Serrano et al. (2017) that considers the risk of different financial resources in the EOQ context and the work of Lusa et al. (2012) which involves the maximum amount of credit that can be borrowed as a financial constraint of a dynamic lot-sizing model. In addition, all extensions considered for the classic lot-sizing model, such as backlogging, perishable inventory and others mentioned in Martínez-Costa et al. (2014) and Brahimi et al. (2017), are all potential directions that can enrich our model. 
For the three classic models proposed in this work, some specific extensions may be respectively studied:

- for $E O Q_{P(W C R)}$ model: as we reveal for this problem, there is a complex trade-off between the multitudes of different operational and financial costs in order to maximize the profit. Based on this trade-off, it could be interesting to establish heuristics for the capacitated lot-sizing problem considering OWCR financing costs. This is inspired by the way how the trade-off in the EOQ problem is used for the capacitated lot-sizing model proposed by Silver \& Meal (1973), the Least Unit Cost heuristic proposed by Gorham (1968) and the Part Period Balancing heuristic proposed by DeMatteis (1968).

- for $U L S_{P(W C R)}$ model: it seems promising to reduce the complexity of this algorithm to $\mathrm{O}\left(n^{3} \log n\right)$ by examining the approaches presented in Wagelmans et al. (1992), Aggarwal \& Park (1993) and Federgruen \& Tzur (1991) that improve the complexity of the WagnerWhitin algorithm. Moreover, future research can be undertaken to consider time-varying cost parameters for this case, such as the Wagner-Whitin cost in the case of the ULS. With this cost, the complexity of the fast backward dynamic programing algorithm of Wagelmans et al. (1992) can be improved from $O(T \log T)$ to $O(T)$.

- for $M L L P_{P(W C R)}$ model: for future research, a generalization of the chain structure seems imperative. The first setup can focus on the assembly structure and then the general structure. Moreover, the development of heuristic or meta-heuristic algorithms helps us to solve problems with a more realistic size and with fewer assumptions. Furthermore, in addition to the two approaches proposed in this thesis, the decentralized approach has drawn increasing attention since the work of the Homberger (2010). The problem concerning the relation (domination) between supply chain members will thus be addressed that involves more negotiation mechanisms and game theory techniques as studied in work of Hovelaque et al. (2015). 



\section{Résumé en français}

\section{Introduction générale}

En période de crise financière, les entreprises ont besoin de trésorerie pour réagir efficacement aux aléas et assurer leur solvabilité. Cette thèse se focalise sur l'intégration de la gestion des opérations et de la finance d'entreprise. Pour ce faire, nous développons des modèles de planification tactique gérant simultanément les flux physiques et financiers. Le coût de financement des opérations basé sur le besoin en fonds de roulement (BFR) est intégré comme un nouvel aspect financier jamais considéré dans la littérature des problèmes de lot-sizing.

Le besoin en fonds de roulement (BFR) correspond au niveau de fonds nécessaire pour financer les activités d'exploitation d'une entreprise, c'est-à-dire pour acheter les matières premières, payer les fournisseurs, configurer et faire tourner les machines, etc. En bref, le besoin en fonds de roulement est généré par les créances des clients et les investissements réalisés à la production, et est réduit par les dettes dues aux fournisseurs. Pendant ces dernières années, la gestion du besoin en fonds de roulement attire de plus en plus l'attention des entreprises en raison de la crise financière. En effet les liquidités sont le fluide vital de toute entreprise et aucune entreprise ne peut survivre sans liquidités. Même si une entreprise réalise des profits substantiels, elle ne peut jamais garantir qu'il reste suffisamment de liquidités disponibles pour répondre aux créances impayées après impôts, dépenses en capital et allocation de dividendes. Donc, dans la pratique, le profit n'est pas automatiquement égal à la trésorerie, et la manière dont une entreprise gère son besoin en fonds de roulement dans un environnement en croissance peut affecter significativement son flux de trésorerie.

Dans cette thèse, cette problèmatique est étudiée dans trois différents cas. Nous proposons d'abord une extension du modèle EOQ intégrant les coûts de financement du BFR, l'objectif etant la maximisation du profit. Une quantité de production optimale est obtenue analytiquement, puis nous proposons l'analyse de la sensibilité du modèle. De plus, les comparaisons avec le modèle EOQ et un modèle qui considère le coût du capital sont étudiées. Ensuite, nous travaillons sur un modèle à demande dynamique. La propriété ZIO est démontrée, elle permet l'utilisation d'un algorithme en temps polynomial. Enfin le cas multi-niveau à capacité infini est étudié avec une approche séquentielle puis centralisée. La propriété ZIO est prouvée pour les deux approches. Des algorithmes de programmation dynamique sont utilisés pour obtenir une solution optimale. Cette thèse peut être considérée comme un premier, mais significatif, travail combinant la planification de production et la gestion du besoin en fond de roulement. Nous montrons que les aspects financiers ont un impact important sur les plans de production. Les cas étudiés dans cette thèse peuvent être 
considérés comme des sous-problèmes dans l'étude de problèmes plus réalistes.

\section{Intégration du coût de financement du BFR dans le modèle de l'EOQ}

Dans ce chapitre, nous établissons un nouveau modèle de maximization du profit basé sur le modèle classique de l'"Economic Order Quantity" (EOQ) en considérant le coût de financement du BFR. Depuis la création du modèle EOQ, il a été largement étudié pour des problèmes de gestion des stocks et est adapté aux cas de plus en plus réalistes. Dans la littérature récente, la valeur actuelle nette et le crédit commercial sont souvent considérés comme un aspect financier intégré dans le modèle EOQ (voir Taleizadeh \& Nematollahi (2014) et Martínez-Costa et al. (2014)). Cependant, aucun de ces modèles ne considère le financement du besoin en fonds de roulement comme nous le faisons. Attendu que le BFR est d'une importance critique car il est directement lié à la solvabilité de l'entreprise et peut générer des coûts financiers importants, ce travail vise à intégrer le coût de financement du BFR dans le modèle classique de l'EOQ. Dans ce cas, nous considérons la planification de la production tactique pour un horizon annuel. Ainsi, l'effet actualisation est négligeable et n'est pas intégrée dans le modèle proposé.

Par conséquent, ce travail introduit une gestion conjointe des flux physiques et financiers en utilisant ce nouveau modèle. Nous nous concentrons sur le problème de la maximisation du profit en tenant compte des coûts opérationnels classiques (achats, configuration, production et détention de stocks) et du coût de financement du BFR associé (par exemple, intérêts sur les prêts). Dans cette étude, seul le BFR lié à l'exploitation (Operating Working Capital Requirement) est pris en compte dans le calcul du coût de financement du BFR. Les taxes, par exemple, ne seront pas prises en compte. Le OWCR est principalement généré par un décalage entre les coûts des activités liées à la production et les revenus de la vente de produits. Dans cette thèse, le BFR est donc le besoin financier pour couvrir les coûts logistiques jusqu'à l'encaissement de paiement du client. La modélisation du BFR dans cette thèse suit le concept du cycle "cash-to-cash". Elle nous permet de suivre la traçabilité des investissements dans le cycle d'exploitation. Pour cette raison, des hypothèses sont prises afin de simplifier et standardiser la modélisation du BFR et surtout pour garantir la traçabilité:

- Les coûts de setup, production et stockage sont payés immédiatement;

- Le BFR n'est financé que par la partie "coût" du revenu;

— Le coût de setup est uniformément reparti sur les produits d'un même lot.

Nous supposons que les paramètres de coût sont constants sur l'horizon dans cette thèse. Une telle modélisation générique nous permet de mesurer l'évolution du BFR sur l'horizon continu de planification. Enfin, le coût de financement du BFR est calculé en multipliant le BFR par le taux d'intérêt.

Après avoir intégré ce coût de financement, le modèle de maximisation du profit basé sur le modèle de l'EOQ est établi. Le profit est défini comme le revenu moins l'ensemble du coût logistique et du coût de financement du BFR. Afin de mettre en évidence l'influence de l'intégration des coûts financiers, la formule analytique de l'EOQ optimale est dérivée avec la méthode Cardan et l'analyse associée est fournie dans ce chapitre. Le résultat démontre qu'une augmentation des coûts unitaires de production et d'achat ou du taux d'intérêt réduit la taille optimale du lot de production lors de la maximisation du profit. De plus, il est démontré que la quantité économique proposée par notre modèle est toujours inférieure à la quantité optimale du modèle EOQ traditionnel. Ainsi, il est profitable de lancer une production plus fréquente de lots plus petits afin de réduire les niveaux de stock, leurs coûts de détention associés et le BFR engendré. De plus, une comparaison avec l'approximation de Jesse et al. (1983) démontre que notre modèle fournit une mesure plus cohérente et plus précise de la quantité de production optimale pour un objectif de maximisation du profit 
intégrant des aspects financiers. Enfin, l'exemple numérique illustre la sensibilité réduite de notre modèle à plusieurs paramètres par rapport au modèle EOQ classique.

Un des résultats importants obtenus avec ce modèle concerne le compromis entre les coûts qui maximise le profit. Pour de futures recherches, ce compromis pourra être utilisé pour établir des heuristiques pour le problème de taille de lot à capacité finie en tenant compte des coûts de financement d'OWCR. Ceci est inspiré de la façon dont le compromis dans le problème EOQ est utilisé pour le modèle de dimensionnement par lot proposé par Silver \& Meal (1973), l'heuristique du moindre coût unitaire proposée par Gorham (1968) et l'heuristique Part Period Balancing proposé par DeMatteis (1968). Enfin, il est démontré que le retard de paiement au fournisseur n'a aucune influence sur la quantité de production optimale. Cependant, il serait intéressant de se pencher sur une relation fournisseur-client à deux échelons car, dans ce contexte, le retard de paiement au fournisseur (comptes créditeurs pour le client) influencerait simultanément les comptes débiteurs du fournisseur. Ainsi, la politique de production du client limiterait la politique du fournisseur et le retard de paiement au fournisseur ne serait plus négligeable.

\section{Modèle de lot-sizing dynamique considérant l'actualisation des coûts et le financement du BFR}

Dans le deuxième chapitre, nous étudions le cas plus realiste à demande variable sur l'horizon de planification en intégrant le coût de financement du BFR. Plus précisément, nous considérons dans ce chapitre le cas mono-site, mono-niveau, big backet et à capacité infinie. Dans la littérature du lot-sizing, ce problème est résolu par le modèle de lot-sizing dynamique, appelé "uncapacitated lot-sizing" (ULS).

Tout d'abord, le modélisation du BFR est modifiée et adaptée au cas discret en fonction de variables désagrégés. Ce type de variable est utilisé dans la formulation "Facility-Location" (FAL) du modèle ULS dans la littérature classique. Ces variables ont été sélectionnées car elles nous permettent d'indiquer les périodes de début et de fin de financement du BFR. Une fois la modélisation définie, elle est intégrée dans le modèle classique de l'ULS avec des contraintes similaires à celles de l'ULS $S_{F A L}$.

L'expression du coût de setup et du BFR induit rend le modèle non-liéaire. En effet, le coût de setup est partagé par tous les produits fabriqués dans un même lot. Ainsi, les méthodes de résolution classiques ne peuvent plus être appliquées pour ce nouveau problème. Afin d'établir une méthode efficace, la propriété Zéro-stock (ZIO) a été prouvée valide pour ce problème. Cette propriété est importante car elle nous permet de réduire significativement le nombre de plans à évaluer parmi lesquels le plan optimal se trouvera. L'idée de la preuve est de montrer que nous arrivons toujours à améliorer la valeur de la fonction objectif d'un plan qui ne respecte pas de propriété ZIO en le transformant à un plan de type ZIO. Grâce à cette propriété, nous sommes capables de reformuler ce problème sous la forme d'un problème de plus court chemin. Le nœud, $V_{t}$, représente la période $t$ de planification avec un noeud fictif à la fin de l'horizon. L'arc, $E_{t k}$, représente une production de l'ensemble des demandes entre la période $t$ et $k-1$. Après avoir reformulé le problème, un algorithme de programmation dynamique est construit en s'inspirant de celui de Wagner et Whitin. La valeur d'un arc peut être calculé au pire en $\mathrm{O}\left(T^{2}\right)$ où $T$ est le longueur de l'horizon. Puis, la récursion est réalisable en $\mathrm{O}\left(T^{2}\right)$. Ainsi, ce problème peut être résolu en $\mathrm{O}\left(T^{4}\right)$.

Enfin, des tests numériques sont effectués afin de démontrer l'influence de l'intégration du coût de financement du BFR dans le modèle classique de l'ULS et l'impact de la valeur des différents paramètres financiers sur le plan optimal de production. Ces tests est réalisés sur une instance basée sur une instance de Trigeiro. Premièrement, le résultat montre que la taille de productions du plan optimal du nouveau problème est généralement plus petite par rapport celle du plan optimal de l'ULS. Cela signifie que nous avons moins intérêt à stocker des produits afin de réduire le coût financement 
du BFR. Deuxièmement, l'impact de variations des paramètres financiers est testé et les résultats confirment ce que nous avons obtenu dans le chapitre précédent à l'exception de l'augmentation du délai de paiement au fournisseur qui a maintenant un impact sur le plan optimale. Ceci est dû à l'intégration de de l'actualisation des coûts.

Pour de futures recherches, il semble prometteur de réduire la complexité de cet algorithme à $\mathrm{O}\left(T^{3} \log T\right)$ en examinant les approches présentées dans Wagelmans et al. (1992), Aggarwal \& Park (1993) et Federgruen \& Tzur (1991) qui améliorent la complexité de l'algorithme de WagnerWhitin. Enfin, de futures recherches peuvent s'intéresser à des paramètres de coûts variables dans le temps cmome les coûts de Wagner-Whitin dans le cas de l'ULS. Avec ce coût, la complexité de l'algorithme de programmation dynamique backward rapide de Wagelmans et al. (1992) peut être améliorée de $O(T \log T)$ à $O(T)$. Afin d'établir une évaluation de la rentabilité globale, un problème de maximisation du profit à deux niveaux (fournisseur-fabricant) sera étudié dans le chapitre suivant. De plus, la capacité de production et la gestion des risques dans la chaîne logistique devraient être pris en compte parmi les autres extensions.

\section{Modèle multi-niveau en considérant le coût de financement du BFR et l'actualisation pour une chaîne logistique en série}

Après avoir étudié les problèmes mono-niveau, nous considérons une chaîne logistique à deux acteurs en série: un fournisseur et un fabricant. Ce dernier organise ses productions pour satisfaire les demandes externes $d_{t}, t \in T$. Pour ce faire, il commande des produits semi-finis auprès du fournisseur. Le fournisseur gère ses activités en respectant les commandes du fabricant, la livraison est soumise à un délai noté $L$. Nous supposons qu'aucun produit semi-fini n'est stocké chez le fabricant. Autrement dit, le fournisseur ne livre les produits semi-finis qu'à la période où le fabricant en a besoin. Chaque transaction est soumise à son propre délai de paiement. Le client final ne paie le fabricant qu'après un délai de paiement qui s'ajoute au délai de livraison, idem entre le fabricant et le fournisseur et entre le fournisseur et son fournisseur en matière première. De plus, dans ce problème, nous considérons également des aspects financiers tels que l'actualisation et le financement du BFR.

La modélisation du BFR est adaptée au cas à deux niveaux. Sachant que l'encaissement des paiements du fabricant au fournisseur est déterminé par le plan de production du fabricant. Par conséquent, le BFR du fournisseur dépend fortement du plan du fabricant. En revanche, les plans de production des deux niveaux peuvent être déduit selon l'objectif prédéfini. Dans cette thèse, deux approches de résolution sont présentées. Premièrement, une approche séquentielle qui représente le cas où le fabricant satisfait la demande externe à profit maximum et le fournisseur organise son activité de production selon le plan optimal de production du fabricant et une approche centralisée qui détermine des plans de production optimaux de chaque niveau en maximisant le profit total de la chaîne.

- Approche séquentielle:

Dans ce cas, nous considérons la planification des deux niveaux comme deux sous-problèmes séparés. Donc, deux étapes de calcul s'effectuent dans cette approche : d'abord, le plan optimal du fabricant peut être calculé en utilisant l'algorithme $U L S_{P(B F R)}$ proposé pour le problème mono-niveau qui est en $\mathrm{O}\left(T^{4}\right)$. Le plan de production ainsi obtenu sera la demande du fournisseur. Une seconde application de l'algorithme nous permet de calculer le plan optimal du fournisseur pour satisfaire les demandes du fabricant. Par conséquent, dans le cas, le complexité du problème est de $\mathrm{O}\left(T^{4}\right)$.

- Approche centralisée

Le problème avec un objectif centralisé a été traité dans la littérature du lot-sizing avec le 
modèle "Multi-Level Lot-sizing Problem" (MLLP). Initialement, il a été étudié par Zangwill (1966) sur un problème avec une nomenclature en série. Pour le résoudre, l'auteur a proposé un algorithme de programmation dynamique. Cependant, cet algorithme ne peut pas être directement appliqué à notre problème. C'est parce que le concept du réseau du flot ne permet pas d'identifier précisément les périodes de début et de fin de financement du BFR. Donc, après avoir prouvé la propriété "zéro-stock" (ZIO) pour ce problème et sous certains hypothèses, nous proposons également un algorithme de programmation dynamique pour le résoudre en $\mathrm{O}\left(T^{6}\right)$. Le principe de l'algorithme est le suivant :

- reformuler le problème sous forme de problème plus court chemin à chaque niveau ;

- considérer le problème au niveau fournisseur comme un problème maître ;

- pour calculer la valeur d'arc au niveau fournisseur, il faut résoudre préalablement un sousproblème au niveau fabricant. Ce sous-problème est entre les mêmes périodes que l'arc considéré au niveau fournisseur. La valeur d'arc du sous-problème comporte non seulement le profit et l'ensemble des coûts logistiques et financiers du fabricant, mais aussi les coûts du fournisseur qui dépendent du plan de production du fabricant (le coût de stockage et le coût de financement du BFR).

- la récursion du problème maître et de chaque sous-problème est similaire que celui du algorithme mono-niveau.

Le calcul du sous-problème est réalisé au pire en $\mathrm{O}\left(T^{4}\right)$ et la récursion du problème maître se réalise en $\mathrm{O}\left(T^{2}\right)$. Ainsi, la complexité du algorithme centralisé est en $\mathrm{O}\left(T^{6}\right)$.

Les tests numériques sont réalisés afin d'illustrer d'abord l'influence des coûts de financement du BFR sur le plan de production de chaque niveau. La taille de lot de production obtenue avec les deux approches est généralement plus petite que celle obtenue avec le modèle MLLP classique. Puis, l'importance du délai de paiement entre les acteurs sur le profit total est identifiée avec l'approche centralisée. Ensuite, le prix unitaire de vente n'impacte pas le plan de production dans le problème mono-niveau. En revanche, nous montrons que le prix unitaire de vente du fournisseur a une influence non négligeable car il est aussi le coût unitaire d'achat du fabricant.

Dans la dernière partie de la thèse, le problème à deux niveau est généralisé à $N$ niveau en série. La modélisation du BFR, le modèle mathématique et les deux approches sont généralisés au cas multi-niveau avec une nomenclature en série. Les complexités sont respectivement $\mathrm{O}\left(N T^{4}\right)$ et $\mathrm{O}\left(N T^{6}\right)$ où $N$ est le nombre de niveaux de la chaîne. Pour l'approche centralisée, trois types de valeur d'arc sont formulés afin de prendre en compte l'interdépendance entre les niveaux.

Comme perspectives, la généralisation de la structure de la chaîne nous permet de traiter le problème plus réaliste. Puis, l'interaction entre les acteurs devrait également être considérée à travers les mécanismes de négociation. 



\section{List of Tables}

3.1 Parameters for OWCR modeling . . . . . . . . . . . . . . . . . . . . . . . . . . . 45

3.2 Analysis of influences of varying parameters on optimal batch size . . . . . . . . . . . 51

3.3 Parameter values for the numerical example . . . . . . . . . . . . . . . . . . . 56

4.1 Parameters . . . . . . . . . . . . . . . . . . . . . . . . . . . 63

4.2 Decision variables $\ldots \ldots \ldots \ldots \ldots$

4.3 Parameters $\ldots \ldots \ldots \ldots \ldots$

$\begin{array}{lll}4.4 & \text { Brief literature review } \ldots \ldots \ldots & \ldots\end{array} \ldots \ldots \ldots$

4.5 Parameters for OWCR modeling . . . . . . . . . . . . . . . . . . . . . . . . 71

4.6 Decision variables for OWCR modeling $\ldots \ldots \ldots$. . . . . . . . . . . . . . . . . . . . . . 71

4.7 Formulation of OWCR components . . . . . . . . . . . . . . . . . . . . . . . . 72

4.8 Numerical example parameter values $\ldots \ldots \ldots$. . . . . . . . . . . . . . . . . . . . . 82

4.9 Differences in number of setups and average inventory level between optimal programs of $U L S_{P(W C R)}$ and $U L S \ldots \ldots \ldots \ldots$. . . . . . . . . . . . . . . . . . . . . . . . . . .

5.1 Parameters $\ldots \ldots \ldots \ldots \ldots \ldots$

5.2 Decision variables $\ldots \ldots \ldots \ldots \ldots$

5.3 Parameters for OWCR modeling in two-level case $\ldots \ldots \ldots$. . . . . . . . . . . . . . . 94

5.4 Decision variables for OWCR modeling in two-level case . . . . . . . . . . . . . . . . . . . 94

5.5 Variations due to the transformation for moving $X_{1 m n}$. . . . . . . . . . . . . . . . . 100

5.6 External demand of the illustrative example . . . . . . . . . . . . . . . . . . . . 108



5.8 Arc values of both levels $\ldots \ldots \ldots \ldots$. . . . . . . . . . . . . . . . . . 109

5.9 Optimal production plans of the illustrative example . . . . . . . . . . . . . . . . . 112

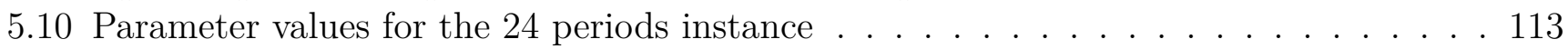

5.11 Comparison of total products held in inventory over time and of the number of setups 114

5.12 Setup timings comparison between optimal programs . . . . . . . . . . . . . . 114

5.13 Comparison of NPVs of financial terms with different $r_{2}$ using the sequential approach 117

5.14 Comparison of NPVs of financial terms with different $r_{2}$ using the centralized approach 117

5.15 Comparison of NPVs of financial terms with different $r_{1}$ using the sequential approach 117

5.16 Comparison of NPVs of financial terms with different $r_{1}$ using the centralized approach 118

5.17 Comparison of NPVs of financial terms with different $r_{0}$ using the sequential approach 119

5.18 Comparison of NPVs of financial terms with different $r_{0}$ using the centralized approach 119

5.19 Comparison of changes with the variations of payment delays using the centralized

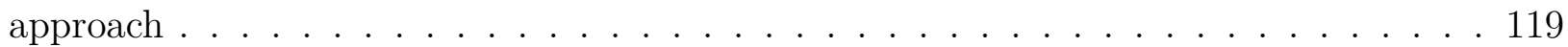

5.20 Comparison of the profit of the supplier, the total cost of the manufacturer and the objective value with different selling prices using the centralized approach . . . . . . . 120 



\section{List of Figures}

2.1 Activities in the logistic process in the supply chain . . . . . . . . . . . . . . . . . 17

2.2 Inter-organizational process in the supply chain . . . . . . . . . . . . . . . . . . 17

2.3 House of supply chain management (Stadtler, 2005) . . . . . . . . . . . . . . . . 20

2.4 Software modules covering the supply chain planning matrix (Meyr et al. (2008)) . . . 20

2.5 Classification of lot-sizing models (Comelli et al. (2008)) $\ldots$. . . . . . . . . . . . 26

2.6 Physical and financial supply chain $\ldots \ldots \ldots \ldots \ldots$

2.7 Increasing number of firms using supply chain finance method in Germany (Wuttke et al. (2016)) $\ldots \ldots \ldots \ldots \ldots \ldots \ldots \ldots \ldots$

2.8 Project RCSM $\ldots \ldots \ldots \ldots \ldots \ldots$

$3.1 \quad$ Illustration of trade-off between costs in basic EOQ model $\ldots$. . . . . . . . . . . . . . 39

\begin{tabular}{ll|l}
\hline 3.2 & Historical evolution in relation to the aspects and assumptions considered in EOQ & \\
\hline & literature (Andriolo et al. $(2014))$ & $\ldots \ldots \ldots$
\end{tabular}

3.3 Illustration of OWCR of purchasing, setup and production costs in a cycle . . . . . . 45

$3.4 \quad$ Illustration of the OWCR calculation for inventory holding cost $\ldots$. . . . . . . . . . . 46

3.5 Comparison of total cost between the EOQ and proposed model . . . . . . . . . . . . 57

3.6 Trade-off between batch size related costs . . . . . . . . . . . . . . . . . . . . . 58

$3.7 \quad$ Variations of optimal batch sizes to $s$ with both models . . . . . . . . . . . . . . . . 58

3.8 Variations of optimal batch sizes with varying $s$ with the models . . . . . . . . . . . . 58

4.1 Physical and financial flows illustration . . . . . . . . . . . . . . . . . . . 70

4.2 Reformulation in shortest path problem . . . . . . . . . . . . . . . . . . . 80

4.3 Optimal production program comparison with fixed parameter values . . . . . . . . . 83

4.4 Comparison of number of setups between optimal plans with different interest rates . 84

$4.5 \quad$ Difference in NPV of total cost including the financing cost . . . . . . . . . . . . . 85

4.6 Difference in logistic cost $\ldots \ldots \ldots \ldots \ldots$. . . . . . . . . . . . . . . . . 85

4.7 Production plan comparison with varying purchasing unit cost and downstream delay 85

4.8 Variation of number of setups with varying production unit cost . . . . . . . . . . 85

$4.9 \quad$ Production plan comparison with varying delays in payment from client . . . . . . . . 86

$5.1 \quad$ Four product BOM structures $\ldots \ldots \ldots$. . . . . . . . . . . . . . . . . 89

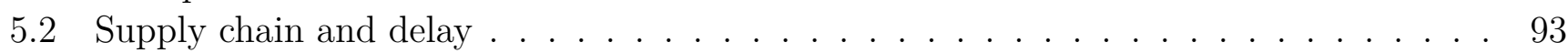

$5.3 \quad$ Sequential approach $\ldots \ldots \ldots \ldots \ldots$

5.4 A node and associated arcs in the Zangwill's network presentation of the multi-level

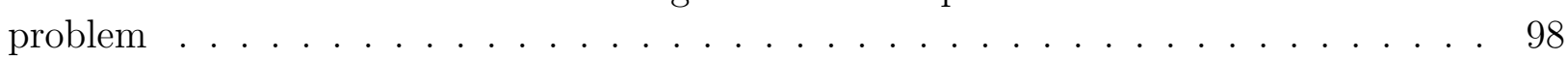

5.5 Centralized approach for $2 U L S_{P(W C R)} \ldots \ldots \ldots \ldots \ldots$. . . . . . . . . . . . 104

5.6 Comparison of optimal production programs of the manufacturer with different

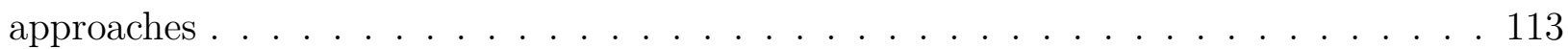

5.7 Comparison of optimal production programs of the supplier with different approaches 114

5.8 Comparison of number of setups between optimal plans with different $\alpha_{0} \quad \ldots \ldots$. . . 115

5.9 Comparison of cumulative inventory between optimal plans with different $\alpha_{0} \ldots \ldots$ 
5.10 Production plan comparison with varying $r_{2} \ldots \ldots \ldots \ldots$. . . . . . . . 117

5.11 Production plan comparison with varying $r_{1} \ldots \ldots \ldots \ldots$. . . . . . . . 118

5.12 Production plan comparison with varying $r_{0} \ldots \ldots \ldots$. . . . . . . . . . . 118

5.13 Comparison of optimal production programs of the manufacturer with different selling prices of the supplier . . . . . . . . . . . . . . . . . 120

5.14 Comparison of optimal production programs of the supplier with different selling prices of the supplier . . . . . . . . . . . . . . . . . . . . . . 121 


\section{Bibliography}

Afentakis, P. \& Gavish, B. (1986), 'Optimal lot-sizing algorithms for complex product structures', Operations research 34(2), 237-249. 90

Afentakis, P., Gavish, B. \& Karmarkar, U. (1984), 'Computationally efficient optimal solutions to the lot-sizing problem in multistage assembly systems', Management Science 30(2), 222-239. 90

Aggarwal, A. \& Park, J. K. (1993), 'Improved algorithms for economic lot size problems', Operations research 41(3), 549-571. 65, 86, 129, 134

Amiti, M. \& Weinstein, D. E. (2011), 'Exports and financial shocks', The Quarterly Journal of Economics 126(4), 1841-1877. 27

Andriolo, A., Battini, D., Grubbström, R. W., Persona, A. \& Sgarbossa, F. (2014), 'A century of evolution from harris's basic lot size model: Survey and research agenda', International Journal of Production Economics 155, 16-38. 39, 40, 41, 42, 139

Arkin, E., Joneja, D. \& Roundy, R. (1989), 'Computational complexity of uncapacitated multiechelon production planning problems', Operations research letters 8(2), 61-66. 90

Babich, V. \& Sobel, M. J. (2004), 'Pre-ipo operational and financial decisions', Management Science 50(7), 935-948. 12, 27

Baglin, G., Bruel, O., Garreau, A., Greif, M., Kerbache, L. \& van Delft, C. (2005), 'Management industriel et logistique: conception et pilotage de la supply chain (4 ème édition)', Economica, Paris . 22

Bahl, H. C., Ritzman, L. P. \& Gupta, J. N. (1987), 'Or practice: Determining lot sizes and resource requirements: A review', Operations Research 35(3), 329-345. 90, 91

Beamon, B. M. (1998), 'Supply chain design and analysis:: Models and methods', International journal of production economics 55(3), 281-294. 16

Benito, A., Neiss, K. S., Price, S. \& Rachel, L. (2010), 'The impact of the financial crisis on supply', Bank of England Quarterly Bulletin p. Q2. 12

Bihlmaier, R., Koberstein, A. \& Obst, R. (2009), 'Modeling and optimizing of strategic and tactical production planning in the automotive industry under uncertainty', OR spectrum 31(2), 311336. 67, 68

Birge, J. R. (2014), 'Om forum - operations and finance interactions', Manufacturing \& Service Operations Management 17(1), 4-15. 12

Biteau, R. \& Biteau, S. (2003), La maîtrise des flux industriels, Éditions d'Organisation. 18

Bitran, G. R. \& Yanasse, H. H. (1982), 'Computational complexity of the capacitated lot size problem', Management Science 28(10), 1174-1186. 25, 62, 128

Blackburn, J. D. \& Millen, R. A. (1982), 'Improved heuristics for multi-stage requirements planning systems', Management Science 28(1), 44-56. 91

Bogataj, M. \& Grubbström, R. W. (2012), 'On the representation of timing for different structures within mrp theory', International Journal of Production Economics 140(2), 749-755. 92 
Bookbinder, J. H. \& Koch, L. A. (1990), 'Production planning for mixed assembly/arborescent systems', Journal of Operations Management 9(1), 7-23. 88, 91

Brahimi, N., Absi, N., Dauzère-Pérès, S. \& Nordli, A. (2017), 'Single-item dynamic lot-sizing problems: An updated survey', European Journal of Operational Research · 25, 26, 62, 90, 128

Brealey, M. (2001), 'Marcus. 2007. fundamentals of corporate finance', International Edition p. 435. 42

Buchmann, P., Roos, A., Jung, U. \& Martin, A. (2008), 'Cash for growth: The neglected power of working-capital management', BCG Opportunities for Actions. Available Online: http://www. bcg. com. cn/en/files/publications/articles_pdf/Cash_for_Growth_May_2008. pdf [Accessed Mai 18 2014] . 11

Buer, T., Homberger, J. \& Gehring, H. (2013), 'A collaborative ant colony metaheuristic for distributed multi-level uncapacitated lot-sizing', International Journal of Production Research 51(17), 5253-5270. 92

Buzacott, J. (1975), 'Economic order quantities with inflation', Operational research quarterly pp. 553-558. 41

Cattrysse, D., Maes, J. \& Van Wassenhove, L. N. (1990), 'Set partitioning and column generation heuristics for capacitated dynamic lotsizing', European Journal of Operational Research 46(1), 38-47. 62

Chang, C.-T., Ouyang, L.-Y., Teng, J.-T. \& Cheng, M.-C. (2010), 'Optimal ordering policies for deteriorating items using a discounted cash-flow analysis when a trade credit is linked to order quantity', Computers \& Industrial Engineering 59(4), 770-777. 41

Chang, C.-T., Wu, S.-J. \& Chen, L.-C. (2009), 'Optimal payment time with deteriorating items under inflation and permissible delay in payments', International Journal of Systems Science 40(10), 985-993. 41

Chauffour, J.-P. \& Farole, T. (2009), 'Trade finance in crisis: market adjustment or market failure?'. 27

Chen, S.-C. \& Teng, J.-T. (2015), 'Inventory and credit decisions for time-varying deteriorating items with up-stream and down-stream trade credit financing by discounted cash flow analysis', European Journal of Operational Research 243(2), 566-575. 46

Chen, T.-L., Lin, J. T. \& Wu, C.-H. (2014), 'Coordinated capacity planning in two-stage thin-filmtransistor liquid-crystal-display (tft-lcd) production networks', Omega 42(1), 141-156. 12

Christopher, M. (1992), Logistics \& supply chain management, Pitman Publishing, London. 15

Christopher, M. (1999), 'Logistics and supply chain management: Strategies for reducing cost and improving service financial times: Pitman publishing. london, 1998 isbn 0273630490 (hardback) $294+1 \times$ pp.'. 92

Clark, A. J. \& Scarf, H. (1960), 'Optimal policies for a multi-echelon inventory problem', Management science 6(4), 475-490. 90

Clark, A. R. \& Armentano, V. A. (1995), 'The application of valid inequalities to the multi-stage lot-sizing problem', Computers \& operations research 22(7), 669-680. 89

Claro, J. \& De Sousa, J. P. (2012), 'A multiobjective metaheuristic for a mean-risk multistage capacity investment problem with process flexibility', Computers \& Operations Research 39(4), 838-849. 66

Colin, J. (2004), 'La logistique du point de vue des sciences de gestion', La logistique, entre management et optimisation, LIÈVRE, P., TCHERNEV, N.,(sous la direction de), Lavoisier, Paris pp. 34-43. 16 
Comelli, M., Gourgand, M. \& Lemoine, D. (2008), 'A review of tactical planning models', Journal of Systems Science and Systems Engineering 17(2), 204-229. 25, 26, 139

Cooper, M. C., Lambert, D. M. \& Pagh, J. D. (1997), 'Supply chain management: more than a new name for logistics', The international journal of logistics management 8(1), 1-14. 15, 17, 18

Crama, Y., Pochet, Y., Wera, Y. et al. (2001), 'A discussion of production planning approaches in the process industry' 23

Croom, S., Romano, P. \& Giannakis, M. (2000), 'Supply chain management: an analytical framework for critical literature review', European journal of purchasing \& supply management 6(1), 67-83. 17

Crowston, W. B. \& Wagner, M. H. (1973), 'Dynamic lot size models for multi-stage assembly systems', Management Science 20(1), 14-21. 90

Dalmia, S. (2008), Financial Supply Chain, Tata McGraw-Hill Education. 27

Davis, E. W. (1975), 'A look at the use of production-inventory techniques: past and present', Production and Inventory management 16(4), 1-19. 87

Dellaert, N. \& Jeunet, J. (2000), 'Solving large unconstrained multilevel lot-sizing problems using a hybrid genetic algorithm', International Journal of Production Research 38(5), 1083-1099. 91

Dellaert, N., Jeunet, J. \& Jonard, N. (2000), 'A genetic algorithm to solve the general multi-level lot-sizing problem with time-varying costs', International Journal of Production Economics 68(3), 241-257. 91, 112

Dellaert, N. P. \& Jeunet, J. (2003), 'Randomized multi-level lot-sizing heuristics for general product structures', European Journal of Operational Research 148(1), 211-228. 91

DeMatteis, J. J. (1968), 'An economic lot-sizing technique, i: The part-period algorithm', IBM systems Journal 7(1), 30-38. 59, 129, 133

Demica, S. S. (2007), 'Steady supplu: The growing role of supply chain finance in a changing world', Demica Report Series . 27

Deroussi, L. \& Lemoine, D. (2009), A particle swarm approach for the mllp, in 'Computers \& Industrial Engineering, 2009. CIE 2009. International Conference on', IEEE, pp. 12-17. 92

Dherment-Férère, I. (2007), Maîtrise d'ouvrage et ERP, processus logistiques, comptables et financiers, édition Lavoisier, Paris. 17, 18

Dudek, G. \& Stadtler, H. (2005), 'Negotiation-based collaborative planning between supply chains partners', European Journal of Operational Research 163(3), 668-687. 92

Eppen, G. D., Gould, F. \& Pashigian, B. P. (1969), 'Extensions of the planning horizon theorem in the dynamic lot size model', Management Science 15(5), 268-277. 65

Eppen, G. D. \& Martin, R. K. (1987), 'Solving multi-item capacitated lot-sizing problems using variable redefinition', Operations Research 35(6), 832-848. 91

Ernst, Y. (2010), 'All tied up', White paper, working capital management report · 12

Ernst, Y. (2012), 'All tied up', White paper, working capital management report · 11

Evans, J. R. (1985), 'An efficient implementation of the wagner-whitin algorithm for dynamic lotsizing', Journal of Operations Management 5(2), 229-235. 64

Fawcett, S. E. \& Magnan, G. M. (2001), Achieving world-class supply chain alignment: benefits, barriers, and bridges, Center for Advanced Purchasing Studies Tempe, AZ. 19

Federgruen, A. \& Tzur, M. (1991), 'A simple forward algorithm to solve general dynamic lot sizing models with $n$ periods in $0(n \log n)$ or 0 (n) time', Management Science 37(8), 909-925. 65. 86, 129, 134 
Féniès, P. (2006), Une méthodologie de modélisation par processus multiples et incrémentiels: application pour l'évaluation des performances de la Supply Chain, PhD thesis, Université Blaise Pascal-Clermont-Ferrand II. 16, 18, 24

Fenies, P., Gourgand, M. \& Tchernev, N. (2004), A framework for supply chain performance evaluation, in 'Congresso Internacional de pesquisa em logistica', Vol. 87, pp. 1-12. 16

Fleischmann, B. (2005), 'Distribution and transport planning', Supply chain management and advanced planning pp. 229-244. 61

Fleischmann, B., Ferber, S. \& Henrich, P. (2006), 'Strategic planning of bmw's global production network', Interfaces 36(3), 194-208. 67,68

Forrester, J. W. (1997), 'Industrial dynamics', Journal of the Operational Research Society 48(10), 1037-1041. 17

Ganeshan, R. \& Harrison, T. P. (1995), 'An introduction to supply chain management', Department of Management Science and Information Systems, Penn State University pp. 1-7. 15

Gao, L.-L., Altay, N. \& Robinson, E. P. (2008), 'A comparative study of modeling and solution approaches for the coordinated lot-size problem with dynamic demand', Mathematical and Computer Modelling 47(11), 1254-1263. 65

Genin, P. (2003), 'Planification tactique robuste avec usage d'un aps. proposition d'un mode de gestion par plan de référence', Mémoire de thèse, Ecole des Mines de Paris, France · 15, 16, 20. 23

Giard, V. (2003), Gestion de la production et des flux: avec CD livre électronique+ Logiciels+ Animations, Economica. 22

Gorham, T. (1968), 'Dynamic order quantities', Production and Inventory Management 9(1), 75-81. 59, 129,133

Goyal, S. K. (1985), 'Economic order quantity under conditions of permissible delay in payments', Journal of the operational research society pp. 335-338. 40

Goyal, S. K. \& Gunasekaran, A. (1990), 'Multi-stage production-inventory systems', European Journal of Operational Research 46(1), 1-20. 90

Grubbström, R. W. (1967), 'On the application of the laplace transform to certain economic problems', Management Science 13(7), 558-567. 92

Grubbström, R. W. (1998), 'A net present value approach to safety stocks in planned production', International Journal of Production Economics 56, 213-229. 92

Grubbström, R. W. (1999), 'A net present value approach to safety stocks in a multi-level mrp system', International Journal of Production Economics 59(1), 361-375. 92

Grubbström, R. W. \& Huynh, T. T. T. (2006), 'Multi-level, multi-stage capacity-constrained production-inventory systems in discrete time with non-zero lead times using mrp theory', International Journal of Production Economics 101(1), 53-62. 92

Grubbström, R. W. \& Wang, Z. (2003), 'A stochastic model of multi-level/multi-stage capacityconstrained production-inventory systems', International Journal of Production Economics 81, 483-494. 92

Gupta, D. \& Wang, L. (2009), 'A stochastic inventory model with trade credit', Manufacturing \& Service Operations Management 11(1), 4-18. 27

Gupta, Y. P. \& Keung, Y. (1990), 'A review of multi-stage lot-sizing models', International Journal of Operations \& Production Management 10(9), 57-73. 91

Han, Y., Tang, J., Kaku, I. \& Mu, L. (2009), 'Solving uncapacitated multilevel lot-sizing problems using a particle swarm optimization with flexible inertial weight', Computers \& Mathematics with Applications 57(11), 1748-1755. 92 
Harris, F. W. (1913), 'How many parts to make at once', Operations Research 38(6), 947-950. 11. 37, 54

Helber, S. (1998), Cash-flow oriented lot sizing in mrp ii systems, in 'Beyond Manufacturing Resource Planning (MRP II)', Springer, pp. 147-183. 11, 66, 67, 68, 92

Herzer, J. (1996), 'Production and operations management-strategic and tactical decisions'. 22

Hoberg, K., Protopappa-Sieke, M. \& Steinker, S. (2017), 'How do financial constraints and financing costs affect inventories? an empirical supply chain perspective', International Journal of Physical Distribution \& Logistics Management 47(6). 43

Hofmann, E. \& Kotzab, H. (2010), 'A supply chain-oriented approach of working capital management', Journal of Business Logistics 31(2), 305-330. 43, 68

Hofmann, E., Maucher, D., Piesker, S. \& Richter, P. (2011), Measures for strengthening internal financing power from a supply chain viewpoint, in 'Ways Out of the Working Capital Trap', Springer, pp. 55-73. 93

Hohmann, C. (2004), Audit combiné qualité/supply chain: sécuriser ses relations clients-fournisseurs, Ed. d'Organisation. 18

Homberger, J. (2008), 'A parallel genetic algorithm for the multilevel unconstrained lot-sizing problem', INFORMS Journal on Computing 20(1), 124-132. 92

Homberger, J. (2010), 'Decentralized multi-level uncapacitated lot-sizing by automated negotiation', 4OR 8(2), 155-180. 92, 125, 129

Homberger, J. \& Gehring, H. (2009), An ant colony optimization approach for the multi-level unconstrained lot-sizing problem, in 'System Sciences, 2009. HICSS'09. 42nd Hawaii International Conference on', IEEE, pp. 1-7. 92

Homberger, J., Gehring, H. \& Buer, T. (2015), Integrating side payments into collaborative planning for the distributed multi-level unconstrained lot sizing problem, in 'System Sciences (HICSS), 2015 48th Hawaii International Conference on', IEEE, pp. 1068-1077. 92

Hovelaque, V., Viviani, J.-L. \& Mansour, M. A. (2015), 'Chaine logistique sous contrainte budgétaire', CIGI . 125, 129

Jaggi, C. K., Goyal, S. \& Goel, S. K. (2008), 'Retailer's optimal replenishment decisions with creditlinked demand under permissible delay in payments', European Journal of Operational Research 190(1), 130-135. 41

Jesse, R. R., Mitra, A. \& Cox, J. F. (1983), 'Eoq formula: Is it valid under inflationary conditions?', Decision Sciences 14(3), 370-374. 42, 53, 59, 127, 132

Jeunet, J. \& Jonard, N. (2005), 'Single-point stochastic search algorithms for the multi-level lot-sizing problem', Computers \& operations research 32(4), 985-1006. 91

Kaku, I., Li, Z. \& Xu, C. (2010), 'Solving large multilevel lot-sizing problems with a simple heuristic algorithm based on segmentation', International Journal of Innovative Computing, Information and Control 6(3), 817-827. 92

Karabuk, S. \& Wu, S. D. (2003), 'Coordinating strategic capacity planning in the semiconductor industry', Operations Research 51(6), 839-849. 66

Kilger, C., Meyr, H. \& Stadtler, H. (2015), Supply chain management and advanced planning: concepts, models, software, and case studies, Springer. 19

Klapper, L. (2006), 'The role of factoring for financing small and medium enterprises', Journal of Banking \& Finance 30(11), 3111-3130. 27

Kouvelis, P. \& Zhao, W. (2011), 'The newsvendor problem and price-only contract when bankruptcy costs exist', Production and Operations Management 20(6), 921-936. 41 
Krarup, J. \& Bilde, O. (1977), 'Plant location, set covering and economic lot size: an o (mn)-algorithm for structured problems', Numerische methoden bei optimierungsaufgaben 3, 155-180. 64

Kuik, R. \& Salomon, M. (1990), 'Multi-level lot-sizing problem: Evaluation of a simulated-annealing heuristic', European Journal of Operational Research 45(1), 25-37. 91, 92

Kuik, R., Salomon, M. \& Van Wassenhove, L. N. (1994), 'Batching decisions: structure and models', European journal of operational research 75(2), 243-263. 24

Kyriakopoulos, K. \& De Ruyter, K. (2004), 'Knowledge stocks and information flows in new product development', Journal of Management Studies 41(8), 1469-1498. 19

Lai, R. K. (2006), 'Inventory and the stock market', Working paper, Harvard University · 43

Lamouri, S. \& Thomas, A. (1999), Optimization of the process of development of the sop, in 'International Conference (CPI'99), Tanger, Maroc', pp. 328-338. 22

Lang, J. C. (2010), Production and operations management: Models and algorithms, Springer. 26. 62

Lee, H. L. \& Billington, C. (1993), 'Material management in decentralized supply chains', Operations research 41(5), 835-847. 15, 16

Lee, H. L., Padmanabhan, V. \& Whang, S. (1997), 'Information distortion in a supply chain: The bullwhip effect', Management science 43(4), 546-558. 19

Lemoine, D. (2008), Modèles génériques et méthodes de résolution pour la planification tactique mono-site et multi-site, PhD thesis, Université Blaise Pascal-Clermont-Ferrand II. 88

Levis, A. A. \& Papageorgiou, L. G. (2004), 'A hierarchical solution approach for multi-site capacity planning under uncertainty in the pharmaceutical industry', Computers \& Chemical Engineering 28(5), 707-725. 67, 68

Lim, J. S., Abdul Manan, Z., Hashim, H. \& Wan Alwi, S. R. (2013b), 'Optimal multi-site resource allocation and utility planning for integrated rice mill complex', Industrial \& Engineering Chemistry Research 52(10), 3816-3831. 68

Lim, J. S., Manan, Z. A., Alwi, S. R. W. \& Hashim, H. (2013a), 'A multi-period model for optimal planning of an integrated, resource-efficient rice mill', Computers \& Chemical Engineering 52, 77-89. 68

Love, S. F. (1972), 'A facilities in series inventory model with nested schedules', Management Science 18(5-part-1), 327-338. 90

Lowe, T. J. \& Schwarz, L. B. (1983), 'Parameter estimation for the eoq lot-size model: Minimax and expected value choices', Naval Research Logistics (NRL) 30(2), 367-376. 38

Lundin, R. A. \& Morton, T. E. (1975), 'Planning horizons for the dynamic lot size model: Zabel vs. protective procedures and computational results', Operations Research 23(4), 711-734. 65

Lusa, A., Martínez-Costa, C. \& Mas-Machuca, M. (2012), 'An integral planning model that includes production, selling price, cash flow management and flexible capacity', International Journal of Production Research 50(6), 1568-1581. 128

Lysons, K. \& Farrington, B. (2006), Purchasing and supply chain management, Pearson Education. 18

Malouche, M. \& Chauffour, J.-P. (2011), Trade finance during the great trade collapse, World Bank Publications. 27

Martínez-Costa, C., Mas-Machuca, M., Benedito, E. \& Corominas, A. (2014), 'A review of mathematical programming models for strategic capacity planning in manufacturing', International Journal of Production Economics 153, 66-85. 42, 66, 92, 128, 132

Melo, R. A. \& Wolsey, L. A. (2010), 'Uncapacitated two-level lot-sizing', Operations Research Letters 38(4), 241-245. 91 
Meyers, P. W. \& Tucker, F. G. (1989), 'Defining roles for logistics during routine and radical technological innovation', Journal of the Academy of Marketing Science 17(1), 73-82. 19

Meyr, H., Wagner, M. \& Rohde, J. (2008), Structure of advanced planning systems, in 'Supply chain management and advanced planning', Springer, pp. 109-115. 20, 139

Min, J., Zhou, Y.-W. \& Zhao, J. (2010), 'An inventory model for deteriorating items under stockdependent demand and two-level trade credit', Applied Mathematical Modelling 34(11), 32733285. 41

Mitra, S., Pinto, J. M. \& Grossmann, I. E. (2014), 'Optimal multi-scale capacity planning for powerintensive continuous processes under time-sensitive electricity prices and demand uncertainty. part i: Modeling', Computers \& Chemical Engineering 65, 89-101. 68

Mo, J., Guimei, C., Ting, F. \& Hong, M. (2014), 'Optimal ordering policies for perishable multi-item under stock-dependent demand and two-level trade credit', Applied Mathematical Modelling 38(9), 2522-2532. 41

Modigliani, F. \& Miller, M. H. (1958), 'The cost of capital, corporation finance and the theory of investment', The American economic review 48(3), 261-297. 12

New, S. J. \& Payne, P. (1995), 'Research frameworks in logistics: three models, seven dinners and a survey', International Journal of Physical Distribution \& Logistics Management 25(10), 60-77. 16

Papageorgiou, L. G., Rotstein, G. E. \& Shah, N. (2001), 'Strategic supply chain optimization for the pharmaceutical industries', Industrial \& Engineering Chemistry Research 40(1), 275-286. 67. 68

Pfohl, H.-C. \& Gomm, M. (2009), 'Supply chain finance: optimizing financial flows in supply chains', Logistics research 1(3-4), 149-161. 17

Pimor, Y. (2001), Logistique: techniques et mise en œuvre, Dunod. 16

Pitakaso, R., Almeder, C., Doerner, K. F. \& Hartl, R. F. (2007), 'A max-min ant system for unconstrained multi-level lot-sizing problems', Computers \& Operations Research 34(9), 2533-2552. 92

Pochet, Y. \& Wolsey, L. A. (2006), Production planning by mixed integer programming, Springer Science \& Business Media. 26, 91

Rota, K., Thierry, C. \& Bel, G. (2001), 'La maîtrise des flux (coordonateur: Jp campagne): Chapitre 5" gestion des flux dans les chaînes logistiques (supply chain management)", ouvrage hermès: Traité systèmes pour l'ingénieur', Hermès · 16

Rumyantsev, S. \& Netessine, S. (2007), 'What can be learned from classical inventory models? a cross-industry exploratory investigation', Manufacturing \& Service Operations Management 9(4), 409-429. 43

Sadlovska, V. (2007), 'The 2008 state of the market in supply chain finance'. 27

Schwarz, L. B. (2008), The economic order-quantity (EOQ) model, In: Building Intuition, Springer. 37, 38, 39

Seifert, D., Seifert, R. W. \& Protopappa-Sieke, M. (2013), 'A review of trade credit literature: Opportunities for research in operations', European Journal of Operational Research 231(2), 245-256. 41

Serrano, A., Kraiselburd, S. \& Oliva, R. (2009), 'Ordering decisions, cost of capital, and the value of the firm', Working paper . 43

Serrano, A., Oliva, R. \& Kraiselburd, S. (2017), 'On the cost of capital in inventory models with deterministic demand', International Journal of Production Economics 183, 14-20. 34, 42, 43 , 56. 128 
Shang, K. H., Song, J.-S. \& Zipkin, P. H. (2009), 'Coordination mechanisms in decentralized serial inventory systems with batch ordering', Management Science 55(4), 685-695. 27

Shapiro, J. (2006), Modeling the supply chain, Nelson Education. 18

Silver, E. A. \& Meal, H. C. (1973), 'A heuristic for selecting lot size quantities for the case of a deterministic time-varying demand rate and discrete opportunities for replenishment', Production and inventory management 14(2), 64-74. 59, 129, 133

Simatupang, T. M. \& Sridharan, R. (2002), 'The collaborative supply chain', The international journal of logistics management 13(1), 15-30. 24

Stadtler, H. (2005), 'Supply chain management and advanced planning-basics, overview and challenges', European journal of operational research 163(3), 575-588. 17, 19

Steinberg, E. \& Napier, H. A. (1980), 'Optimal multi-level lot sizing for requirements planning systems', Management Science 26(12), 1258-1271. 89, 90

Straube, F. \& Beyer, I. (2006), Decentralized planning in global and local networks-coordination of inter-and intraorganizational networks at tactical level, in 'Proceedings of IFSAM VIIIth World Congress', pp. 28-30. 92

Taleizadeh, A. A. \& Nematollahi, M. (2014), 'An inventory control problem for deteriorating items with back-ordering and financial considerations', Applied Mathematical Modelling 38(1), 93109. 42 , 132

Tang, O. (2004), 'Simulated annealing in lot sizing problems', International Journal of Production Economics 88(2), 173-181. 91

Tanrisever, F., Cetinay, H., Reindorp, M. \& Fransoo, J. (2012), 'Value of reverse factoring in multistage supply chains', Available at SSRN: 2183991. 27

Tchernev, N. (1997), Modélisation du processus logistique dans les systèmes flexibles de production, PhD thesis. 16, 18, 21

Tchernev, N. (2003), 'Approvisionnement et logistique', Manuel de l'étudiant, Institut Universitaire Professionnalisé Management et gestion des entreprises, Université d'Auvergne · 22

Thangam, A. (2012), 'Optimal price discounting and lot-sizing policies for perishable items in a supply chain under advance payment scheme and two-echelon trade credits', International Journal of Production Economics 139(2), 459-472. 27

Theodore Farris, M. \& Hutchison, P. D. (2002), 'Cash-to-cash: the new supply chain management metric', International Journal of Physical Distribution \& Logistics Management 32(4), 288-298. 43, 68

Thierry, C., Le Page, P., Chapeaublanc, N. \& Bel, G. (1994), Multi-site planning: A centralized or a distributed approach?, in '11th International Conference on Analysis and Optimization of Systems Discrete Event Systems', Springer, pp. 609-615. 24

Thizy, J. \& Van Wassenhove, L. (1983), 'A subgradient algorithm for the multi-item capacitated lot-sizing problem', IIE Transactions. 18 pp. 114-123. 62

Thomas, B. G. \& Bollapragada, S. (2010), 'General electric uses an integrated framework for product costing, demand forecasting, and capacity planning of new photovoltaic technology products', Interfaces 40(5), 353-367. 67, 68

Trigeiro, W. W., Thomas, L. J. \& McClain, J. O. (1989), 'Capacitated lot sizing with setup times', Management science 35(3), 353-366. 82

Trippi, R. R. \& Lewin, D. E. (1974), 'A present value formulation of the classical eoq problem', Decision Sciences 5(1), 30-35. 41

Van Hoesel, S., Romeijn, H. E., Morales, D. R. \& Wagelmans, A. P. (2005), 'Integrated lot sizing in serial supply chains with production capacities', Management science 51(11), 1706-1719. 91 
Veinott Jr, A. F. (1969), 'Minimum concave-cost solution of leontief substitution models of multifacility inventory systems', Operations Research 17(2), 262-291. 90

Veral, E. A. \& LaForge, R. L. (1985), 'The performance of a simple incremental lot-sizing rule in a multilevel inventory environment', Decision Sciences 16(1), 57-72. 91

Vollmann, T. E., Berry, W. L. \& Whybark, D. C. (1997), Manufacturing planning and control systems, Irwin/McGraw-Hill. 22

Vowles, A. (1995), 'Chain of demand', CMA Magazine 69(7), 15-19. 16

Wagelmans, A., Van Hoesel, S. \& Kolen, A. (1992), 'Economic lot sizing: an o (n log n) algorithm that runs in linear time in the wagner-whitin case', Operations Research 40(1-supplement-1), S145S156. 64, 65, 86, 129, 134

Wagner, H. M. \& Whitin, T. M. (1958), 'Dynamic version of the economic lot size model', Management science 5(1), 89-96. 11, 26, 62, 65, 74

Wang, K.-J., Wang, S.-M. \& Yang, S.-J. (2007b), 'A resource portfolio model for equipment investment and allocation of semiconductor testing industry', European Journal of Operational Research 179(2), 390-403. 67, 68

Wang, S., Chen, J. \& Wang, K.-J. (2007a), 'Resource portfolio planning of make-to-stock products using a constraint programming-based genetic algorithm', Omega 35(2), 237-246. 67, 68

Wight, O. (1995), Manufacturing resource planning: MRP II: unlocking America's productivity potential, John Wiley \& Sons. 23

Wu, J., Al-Khateeb, F. B., Teng, J.-T. \& Cárdenas-Barrón, L. E. (2016), 'Inventory models for deteriorating items with maximum lifetime under downstream partial trade credits to creditrisk customers by discounted cash-flow analysis', International Journal of Production Economics 171, 105-115. 12

Wuttke, D. A., Blome, C., Heese, H. S. \& Protopappa-Sieke, M. (2016), 'Supply chain finance: Optimal introduction and adoption decisions', International Journal of Production Economics 178, 72-81. 29, 139

Wuttke, D. A., Blome, C. \& Henke, M. (2013), 'Focusing the financial flow of supply chains: An empirical investigation of financial supply chain management', International Journal of Production Economics 145(2), 773-789. 27

Xiao, Y., Zhang, R., Zhao, Q., Kaku, I. \& Xu, Y. (2014), 'A variable neighborhood search with an effective local search for uncapacitated multilevel lot-sizing problems', European Journal of Operational Research 235(1), 102-114. 92

Yang, S.-J. S., Yang, F.-C., Wang, K.-J. \& Chandra, Y. (2009), 'Optimising resource portfolio planning for capital-intensive industries under process-technology progress', International Journal of Production Research 47(10), 2625-2648. 67, 68

You, F., Grossmann, I. E. \& Wassick, J. M. (2010), 'Multi-site capacity, production, and distribution planning with reactor modifications: Milp model, bilevel decomposition algorithm versus lagrangean decomposition scheme', Industrial \& Engineering Chemistry Research 50(9), 48314849. 68

Zabel, E. (1964), 'Some generalizations of an inventory planning horizon theorem', Management Science 10(3), 465-471. 65

Zangwill, W. I. (1966), 'A deterministic multiproduct, multi-facility production and inventory model', Operations Research 14(3), 486-507. 90, 97, 121, 135

Zeballos, A. C., Seifert, R. W. \& Protopappa-Sieke, M. (2013), 'Single product, finite horizon, periodic review inventory model with working capital requirements and short-term debt', Computers \& Operations Research 40(12), 2940-2949. 30, 43 
Zhang, M., Küçükyavuz, S. \& Yaman, H. (2012), 'A polyhedral study of multiechelon lot sizing with intermediate demands', Operations Research 60(4), 918-935. 91

Zhao, L. \& Huchzermeier, A. (2015), 'Operations-finance interface models: A literature review and framework', European Journal of Operational Research 244(3), 905-917. 12

Zipkin, P. H. (2000), Foundations of inventory management. 43 

Thèse de Doctorat

\title{
Yuan BIAN
}

\author{
Planification tactique de production des flux physiques et financiers d'une \\ chaîne logistique multi-site
}

\section{Tactical production planning for physical and financial flows for supply chain in a multi-site context}

\section{Résumé}

En période de crise financière, les entreprises ont besoin de trésorerie pour réagir efficacement aux aléas et assurer leur solvabilité. Cette thèse se situe à l'intersection entre l'opérationnel et la finance pour développer des modèles de planification tactique gérant simultanément les flux physiques et financiers. Le coût de financement des opérations basé sur le besoin en fond de roulement (BFR) est intégré comme un nouvel aspect financier jamais considéré dans la littérature des problèmes de lot-sizing. Nous proposons une extension du modèle EOQ intégrant les coûts de financement du BFR, l'objectif etant la maximisation du profit. Une quantité de production optimale est obtenue anaIytiquement, puis nous proposons l'analyse de la sensibilité du modèle. De plus, les comparaisons avec le modèle $E O Q$ et un modèle qui considère le coût du capital sont étudiées. Ensuite, nous travaillons sur un modèle à demande dynamique. La propriété ZIO est démontrée, elle permet l'utilisation d'un algorithme en temps polynomial. Enfin le cas multi-niveau à capacité infini est étudié avec une approche séquentielle puis centralisée. La propriété ZIO est prouvée dans ces deux cas. Des algorithmes de programmation dynamique sont utilisés pour obtenir une solution optimale. Cette thèse peut être considérée comme un premier, mais significatif, travail combinant la planification de production et la gestion du besoin en fond de roulement. Nous montrons que les aspects financiers ont un impact important sur les plans de production. Les cas étudiés dans cette thèse peuvent être considérés comme des sousproblèmes dans l'étude de problèmes plus réalistes.

\section{Mots clés}

Lot-sizing, Besoin en Fond de Roulement, Flux de trésorerie actualisés, Maximisation du profit.

\section{Abstract}

In financial crisis, companies always need free cash flow to efficiently react to any uncertainties to ensure solvency. Thus, this thesis serves as an interface between operations and finance to develop tactical production planning models for joint management of physical and financial flows in the supply chain. In these models, the financing cost of operation-based working capital requirement (WCR) is integrated as a new financial aspect never before considered in the lot-sizing literature. We first focus on extending the classic EOQ model by considering the financing cost of WCR with a profit maximization objective. The optimal analytic production quantity formula is derived as well as sensitivity analysis of this model. Moreover, a comparison with the EOQ model and with the formula which considers the cost of capital are discussed. Secondly, a dynamic lot-sizing-based, discounted cash flow model is established based on Uncapacitated lot-sizing model. The zero-inventory ordering property is proven valid for this case and a polynomial-time algorithm can thus be established. Thirdly, multi-level and infinite capacity scenario is investigated with both sequential and centralized approaches. The ZIO property is demonstrated valid in both cases. Dynamic-programming based algorithms are constructed in order to obtain an optimal solution. This thesis should be considered as a first, but significant setup of combining production planning and working capital management. It is shown the significant financial consequences of lot-sizing decision on production planning. The cases investigated in this thesis may be tackled as subproblems in the study of more realistic scenarios.

\section{Key Words}

Lot-sizing , Working Capital Requirement, Discounted cash flow, Profit maximization. 